\title{
Supramolecular Catch and Release
}

Cyclodextrin-Functionalized Microfluidics for Reversible Immobilization of (Model) Bacteria

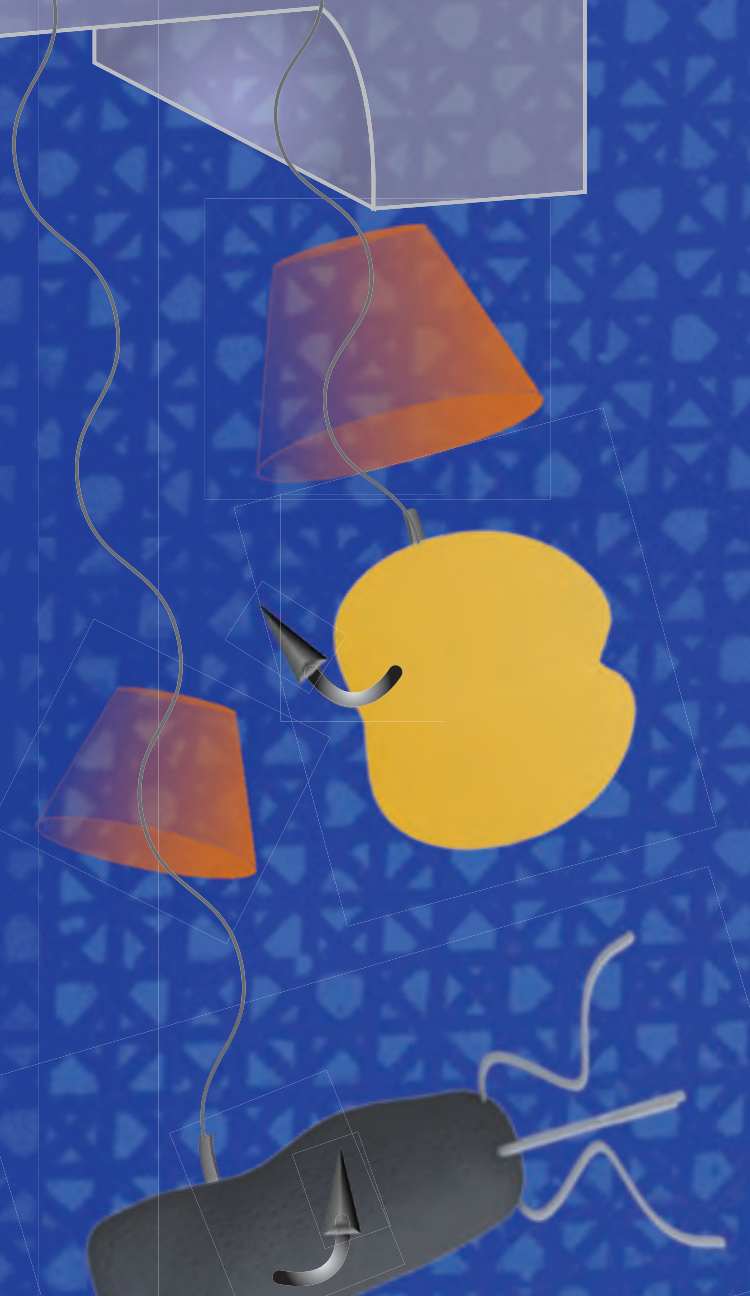





\section{Supramolecular Catch and Release}

Cyclodextrin-Functionalized Microfluidics for Reversible Immobilization of (Model) Bacteria 


\section{Thesis committee}

\section{Promotor}

Prof. Dr A.H. Velders

Professor of BioNanoTechnology

Wageningen University \& Research

\section{Co-promotors}

Dr V. Saggiomo

Assistant professor, BioNanoTechnology

Wageningen University \& Research

Dr F.W.B. van Leeuwen

Associate professor, Department of Radiology

Leiden University Medical Center

\section{Other members}

Prof. Dr H.H.M. Rijnaarts, Wageningen University \& Research

Dr J.A. Dijksman, Wageningen University \& Research

Dr R. Luttge, Eindhoven University of Technology

Dr P.M.F. van der Maas, Hogeschool Van Hall Larenstein, Leeuwarden

This research was conducted under the auspices of the Graduate School for Socio-Economic and Natural Sciences of the Environment (SENSE). 


\section{Supramolecular Catch and Release}

Cyclodextrin-Functionalized Microfluidics for Reversible Immobilization of (Model) Bacteria

\section{Stan B.J. Willems}

\section{Thesis}

submitted in fulfilment of the requirements for the degree of doctor at Wageningen University

by the authority of the Rector Magnificus

Prof. Dr A.P.J. Mol,

in the presence of the

Thesis Committee appointed by the Academic Board

to be defended in public

on Friday 7 February 2020

at 1:30 p.m. in the Aula. 
Stan B.J. Willems

Supramolecular Catch and Release

Cyclodextrin-Functionalized Microfluidics for Reversible Immobilization of (Model) Bacteria 163 pages

$\mathrm{PhD}$ thesis, Wageningen University, Wageningen, the Netherlands (2020)

With references, with summary in English

ISBN: 978-94-6395-256-9

DOI: https://doi.org/10.18174/509854 


\section{Supramolecular Catch and Release}

Cyclodextrin-Functionalized Microfluidics for Reversible Immobilization of (Model) Bacteria

\section{Table of Contents:}

Chapter 1 Introduction $\quad 1$

1.1 Preventing the spread of pathogenic bacteria 2

1.2 Supramolecular platforms for pathogen removal $\quad 4$

$\begin{array}{ll}1.3 \text { Experimental setup } & 13\end{array}$

1.4 Motivation and outline of this thesis 16

$\begin{array}{ll}1.5 \text { References } & 18\end{array}$

Chapter 2 Covalently Bound Monolayers Obtained by Plasma Etching on Glass Surfaces 23

$\begin{array}{ll}2.1 \text { Introduction } & 24\end{array}$

$\begin{array}{ll}2.2 \text { Experimental Section } & 26\end{array}$

2.3 Results and Discussion 29

2.4 Conclusion 33

$\begin{array}{ll}2.5 \text { References } & 34\end{array}$

2.6 Supplementary Figures $\quad 36$

Chapter 3 Plasma Etching for Fabricating Microfluidic Devices with Patterned Monolayers 41

$\begin{array}{ll}3.1 \text { Introduction } & 42\end{array}$

$\begin{array}{ll}3.2 \text { Experimental Section } & 45\end{array}$

$\begin{array}{ll}3.3 \text { Results and Discussion } & 50\end{array}$

$\begin{array}{ll}3.4 \text { Conclusion } & 54\end{array}$

$\begin{array}{ll}3.5 \text { References } & 55\end{array}$

3.6 Supplementary Figures $\quad 58$

Chapter 4 On-Flow Immobilization of Polystyrene Microspheres on $\beta$-Cyclodextrin Patterned

Silica Surfaces through Supramolecular Host-Guest interactions 
4.1 Introduction

$\begin{array}{ll}\text { 4.2 Experimental Section } & 67\end{array}$

$\begin{array}{ll}4.3 \text { Results and Discussion } & 72\end{array}$

$\begin{array}{ll}4.4 \text { Conclusion } & 85\end{array}$

$\begin{array}{ll}4.5 \text { References } & 86\end{array}$

$\begin{array}{ll}\text { 4.6 Supplementary Figures } & 89\end{array}$

Chapter 5 Supramolecular Immobilization of Bacteria in Microfluidic Systems In Presence or $\begin{array}{ll}\text { Absence of an Adamantane Functionalized Peptide } & 107\end{array}$

$\begin{array}{ll}5.1 \text { Introduction } & 108\end{array}$

$\begin{array}{ll}5.2 \text { Experimental Section } & 111\end{array}$

$\begin{array}{ll}5.3 \text { Results and Discussion } & 115\end{array}$

$\begin{array}{ll}5.4 \text { Conclusion } & 119\end{array}$

$\begin{array}{lr}5.5 \text { References } & 120\end{array}$

$\begin{array}{ll}\text { 5.6 Supplementary Figures } & 123\end{array}$

$\begin{array}{ll}\text { Chapter } 6 \text { General Discussion } & 125\end{array}$

6.1 Main Findings 126

6.2 Alternative Surface Functionalizations for Immobilization of Cells 133

6.3 Feasibility for Implementation in Wastewater Treatment Plants 138

6.4 Summarizing Main Concluding Remarks 141

6.5 References $\quad 142$

$\begin{array}{ll}\text { Chapter } 7 \text { Summary } & 145\end{array}$

$\begin{array}{ll}\text { Acknowledgements } & 151\end{array}$

$\begin{array}{ll}\text { About the author } & 157\end{array}$

$\begin{array}{ll}\text { List of publications } & 159\end{array}$

$\begin{array}{ll}\text { Overview of completed training activities (SENSE) } & 161\end{array}$ 


\section{Chapter 1}

\section{General Introduction}

In this chapter, background information is provided on the concepts, buildings blocks, technology, and experimental setups used in this thesis. The increase in prevalence of pathogenic bacteria coupled with development of antibiotic resistance provided the motivation for developing and studying platforms for specific on-flow immobilization of bacteria. To approach this goal, we studied the concept of using supramolecular host-guest chemistry for immobilizing model microparticles and bacteria with cyclodextrin functionalized glass surfaces. Fluorescence microscopy was used as the main tool for characterizing surface functionalization and studying surface adhesion interactions with molecules, particles and bacteria. At the end of this chapter, an outline of the thesis is given. 


\subsection{Preventing the spread of pathogenic bacteria}

Detection and removal of (micro)contaminants from water environments is of increasing interest within agriculture and waste-water treatment. In light of this, developing (bio)sensors for targeting microcontaminants such as bacteria is an important step in order to prevent spreading of pathogenic infections. Bacteria were among the first life forms on Earth and now constitute an extremely large portion of the Earth's inhabitants at a population of $5 \mathrm{x}$ $10^{30}$, easily outnumbering plants and animals. ${ }^{1,2}$ Not all bacteria are purely pathogenic and animal life is dependent on bacteria for their survival, especially in terms of vitamin B12 synthesis. ${ }^{3}$ However, on the other side of the spectrum, pathogenic bacteria such as Yersinia pestis caused deadly diseases like the bubonic plague in the Middle Ages, which is still present in some parts of the world today. Other bacterial diseases include cholera, syphilis, and leprosy. Respiratory infections such as tuberculosis is also caused by pathogenic bacteria, which still kills about 2 million people per year. ${ }^{4}$

The discovery of penicillin by Alexander Fleming in 1929 was an important step in fighting bacterial infections in the modern world, ${ }^{5}$ and novel antibiotics are constantly being developed to prevent and cure microbial infections in humans and animals. However, overuse of antibiotics occurred especially within human healthcare and livestock farming, due to their highly effective treatment of bacterial infections. This accelerated bacterial evolution to develop into antibiotic resistant bacteria (ARB), e.g. methicillin resistant Staphylococcus aureus (MRSA). ${ }^{6}$ Antibiotic resistance can spread as easily as bacteria when antibiotics, ARB, and genes encoding for antibiotic resistance are discharged into environmental reservoirs. Therefore, removal of these bacteria at the source, such as waste-water treatment plants, before discharge is an environmental approach to fight spread of pathogenic bacteria and ABR.

Water is generally the major channel through which bacteria can spread. In order to generate clean water, waste-water treatment plants are crucial for filtration of debris and removal of contaminants. The growing issue of ARB can be tackled by using an environmental approach, which comprises several steps. Firstly, it is important to map antibiotic resistant genes causing public health problems. Following from this information, the role of biological wastewater treatment plants in the spread of antibiotic resistance should be researched. From the agricultural perspective, the impact of manure application on the spread of antibiotic resistance from livestock production can be studied. Lastly, it is also important that new 


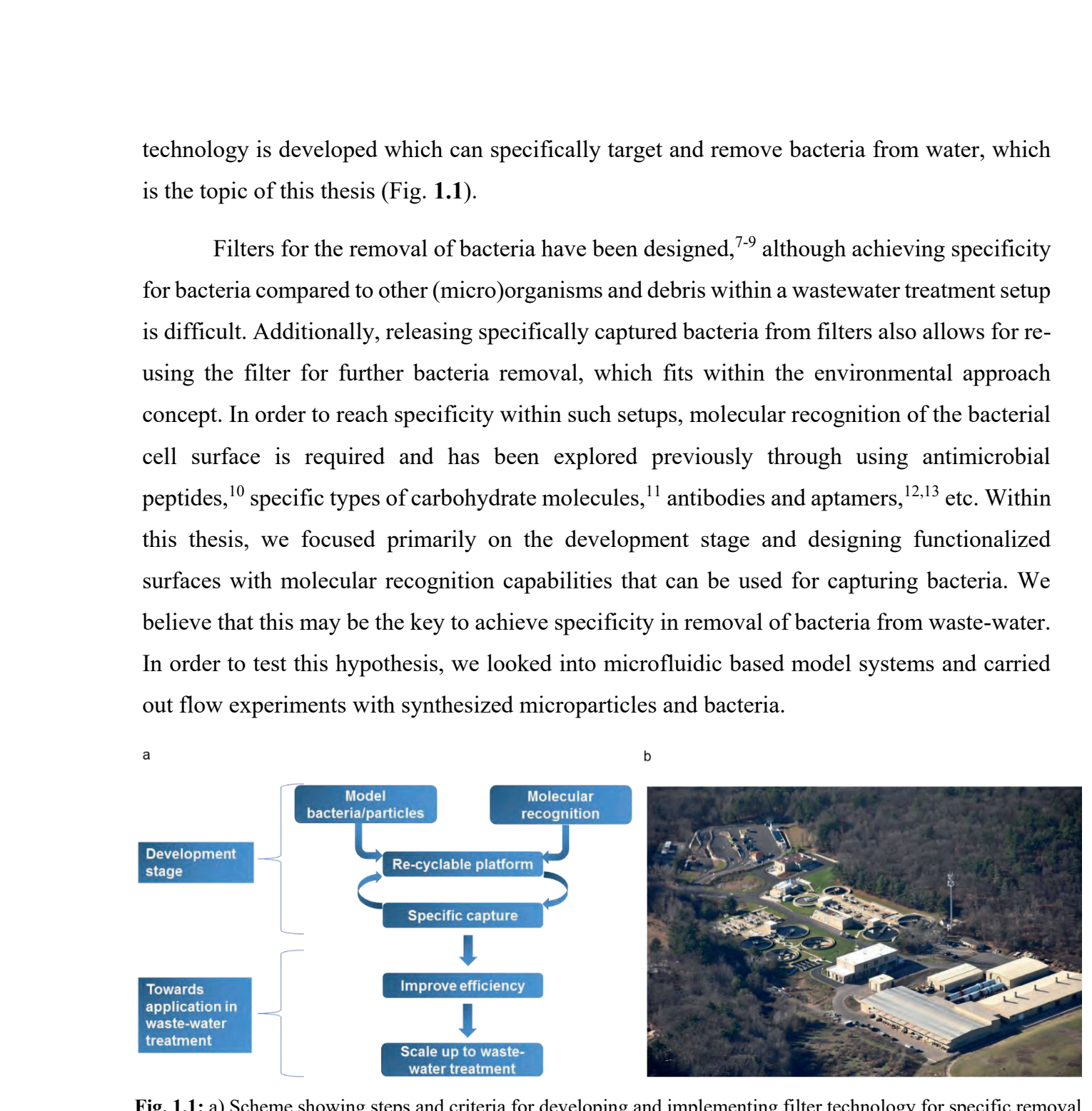

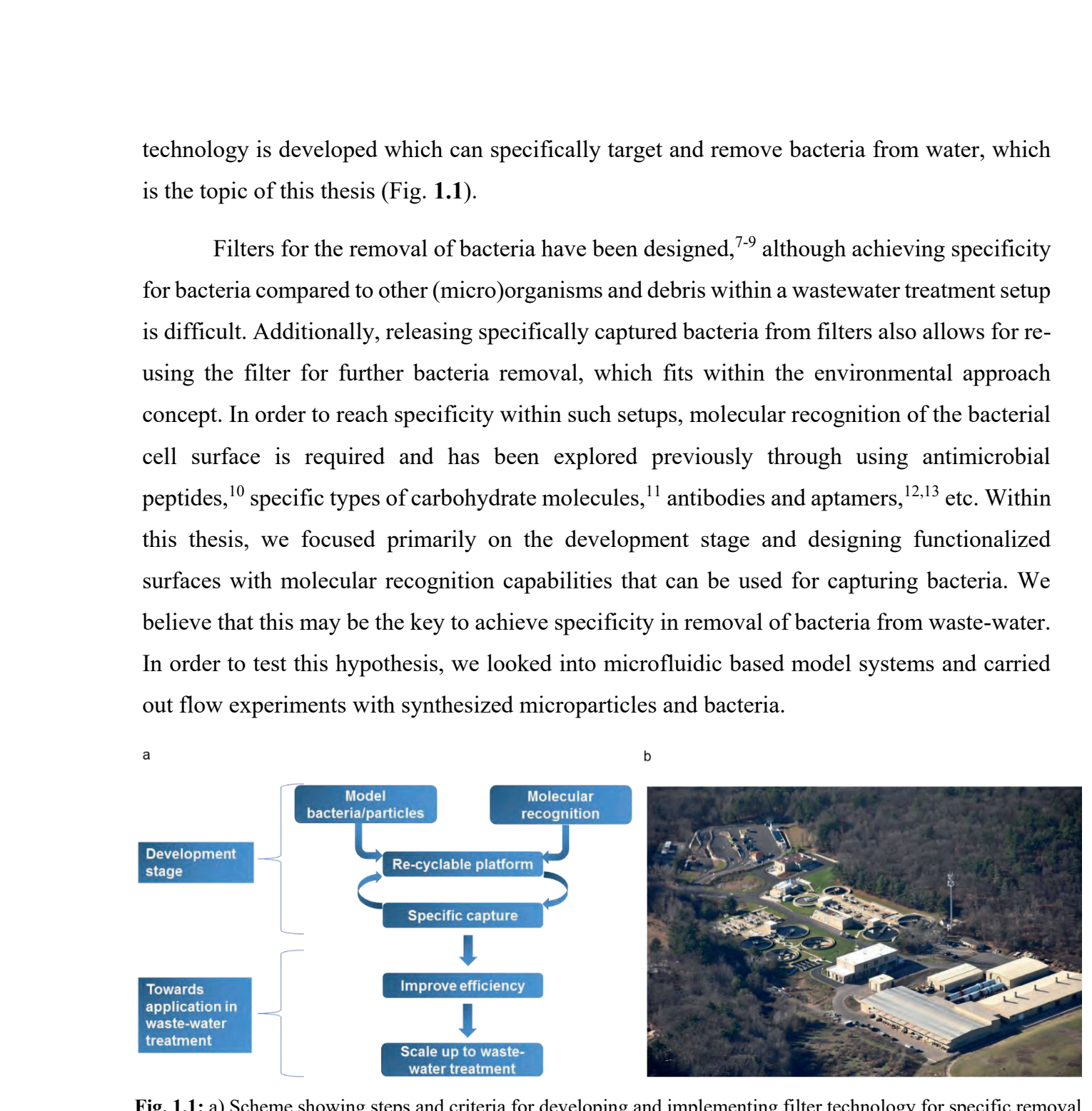

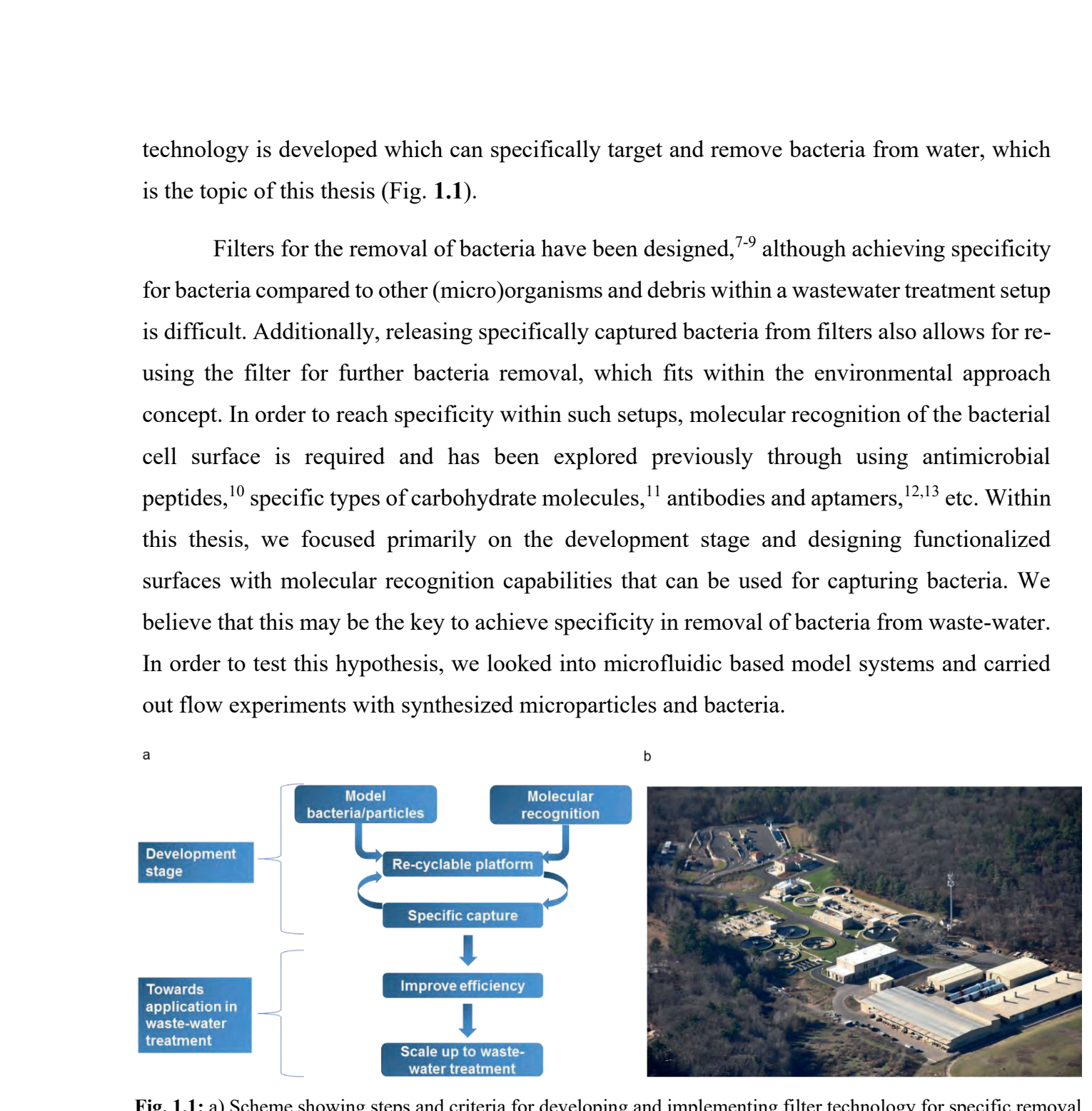

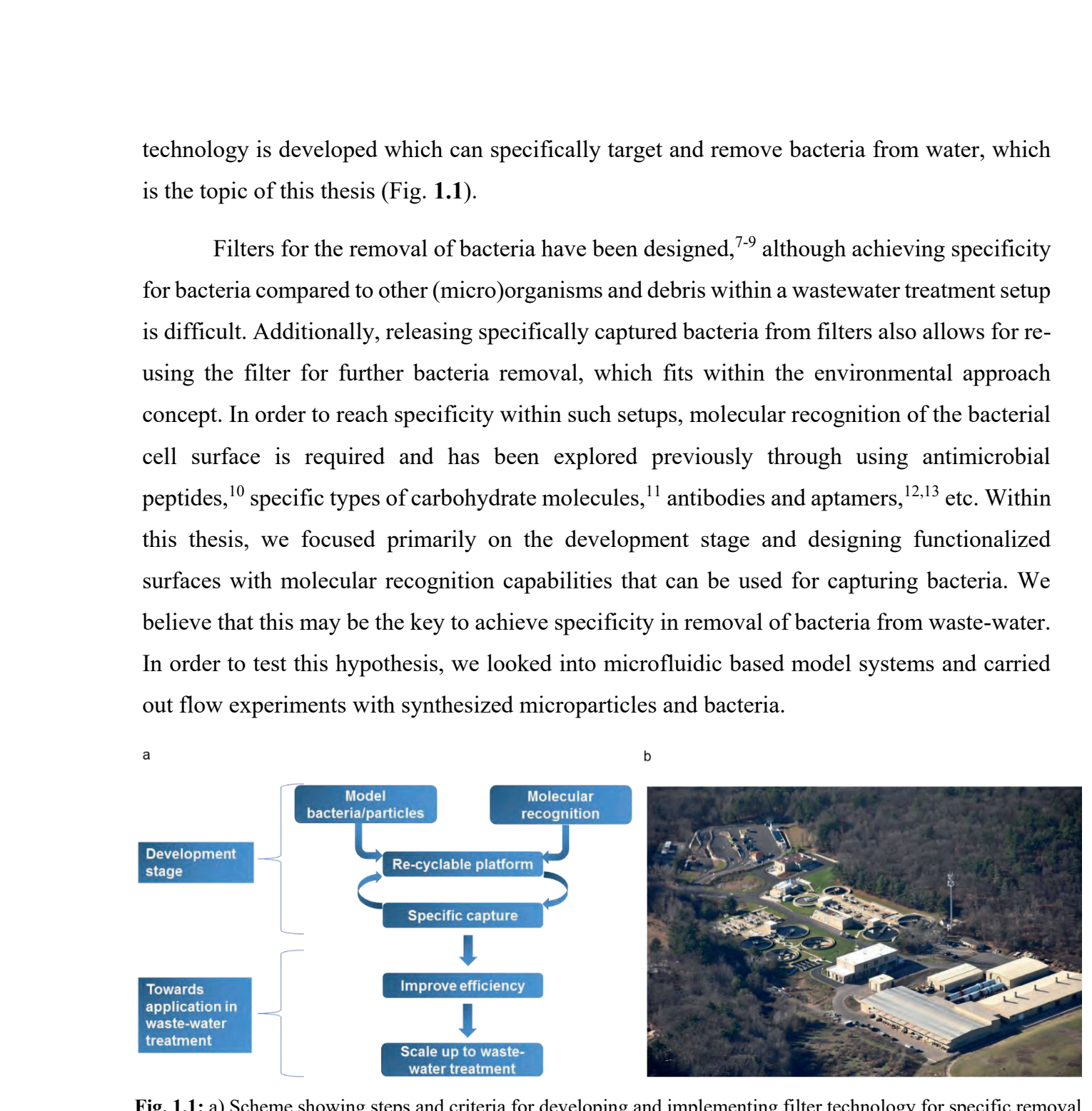

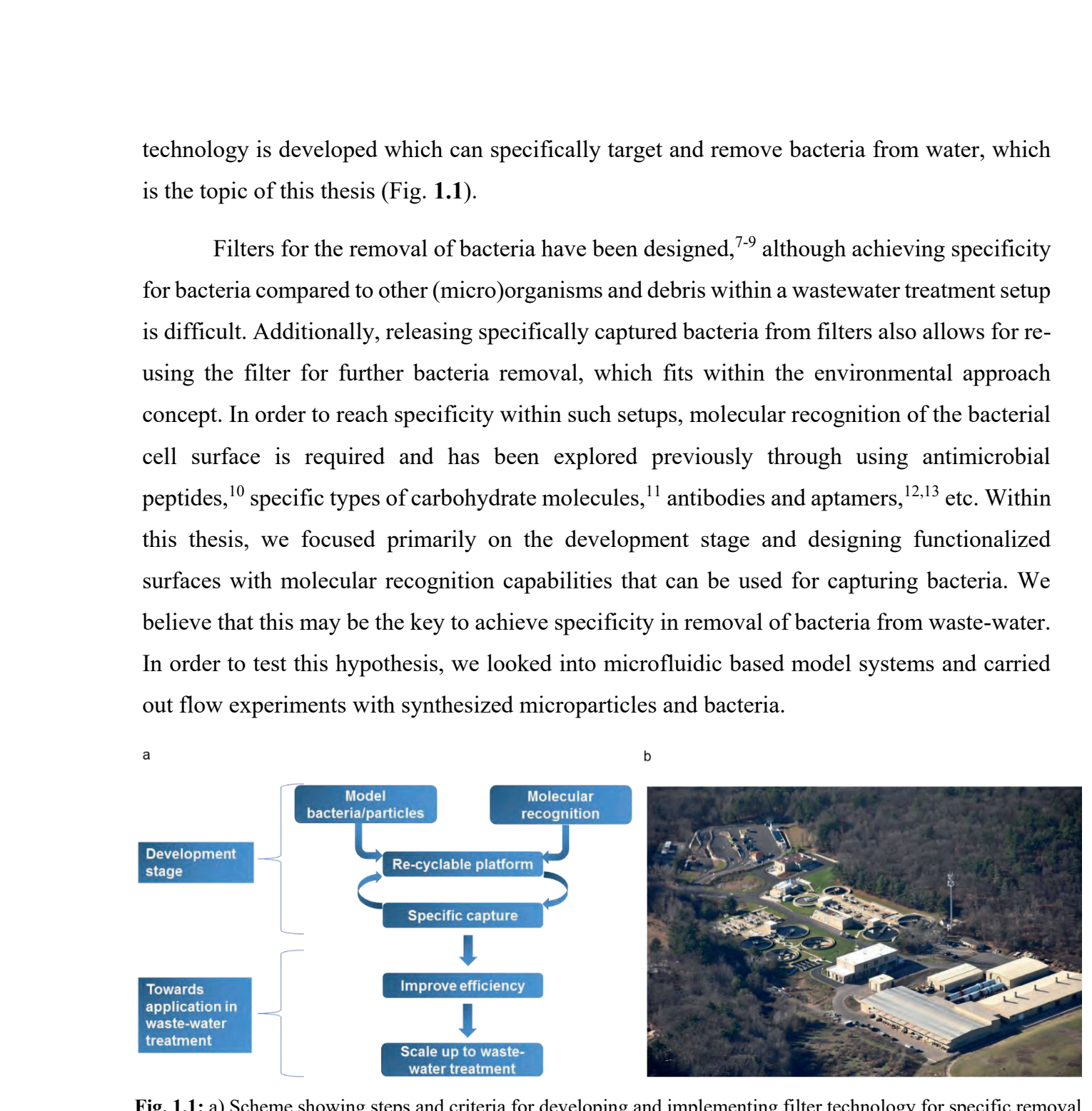

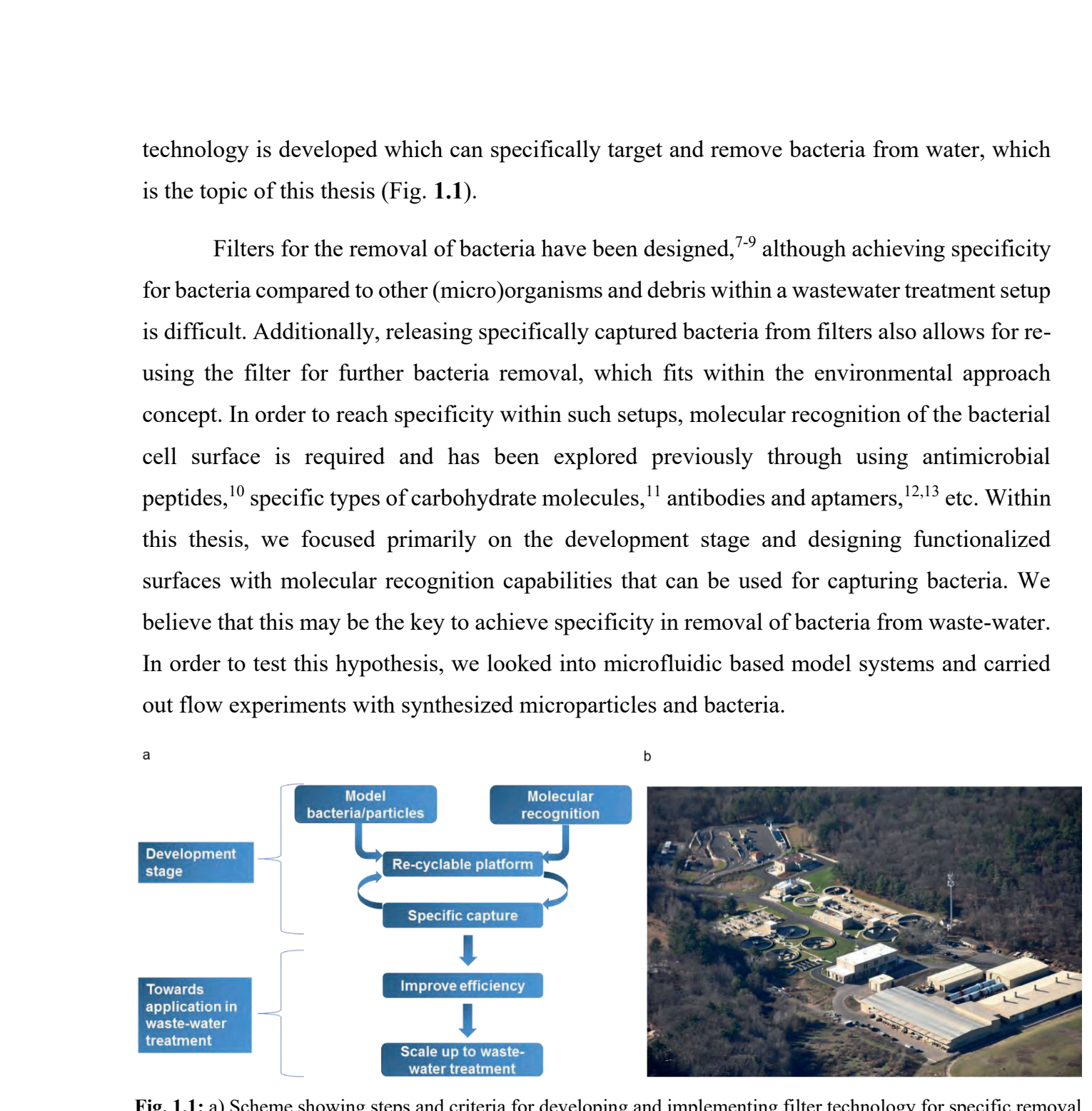

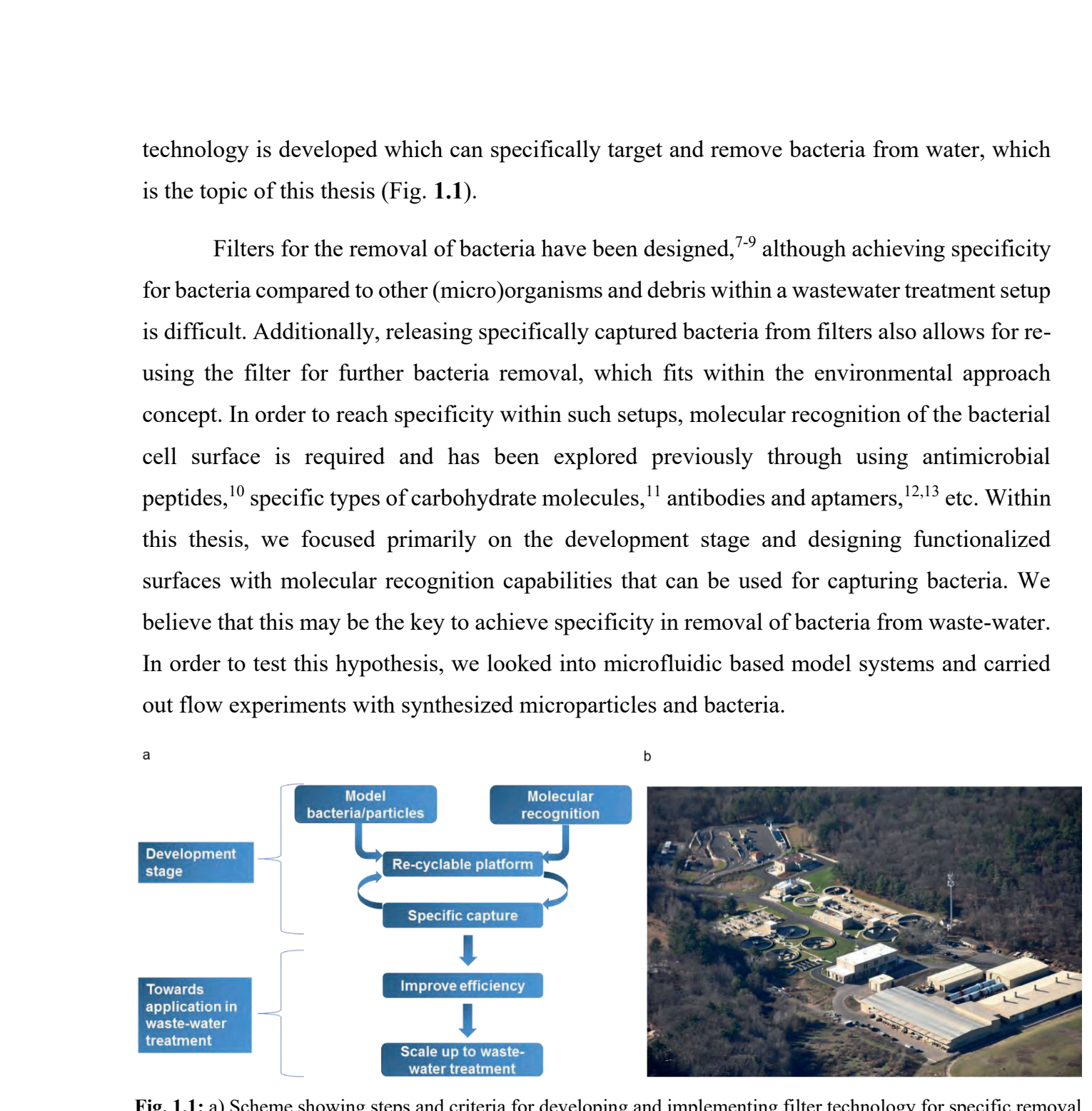

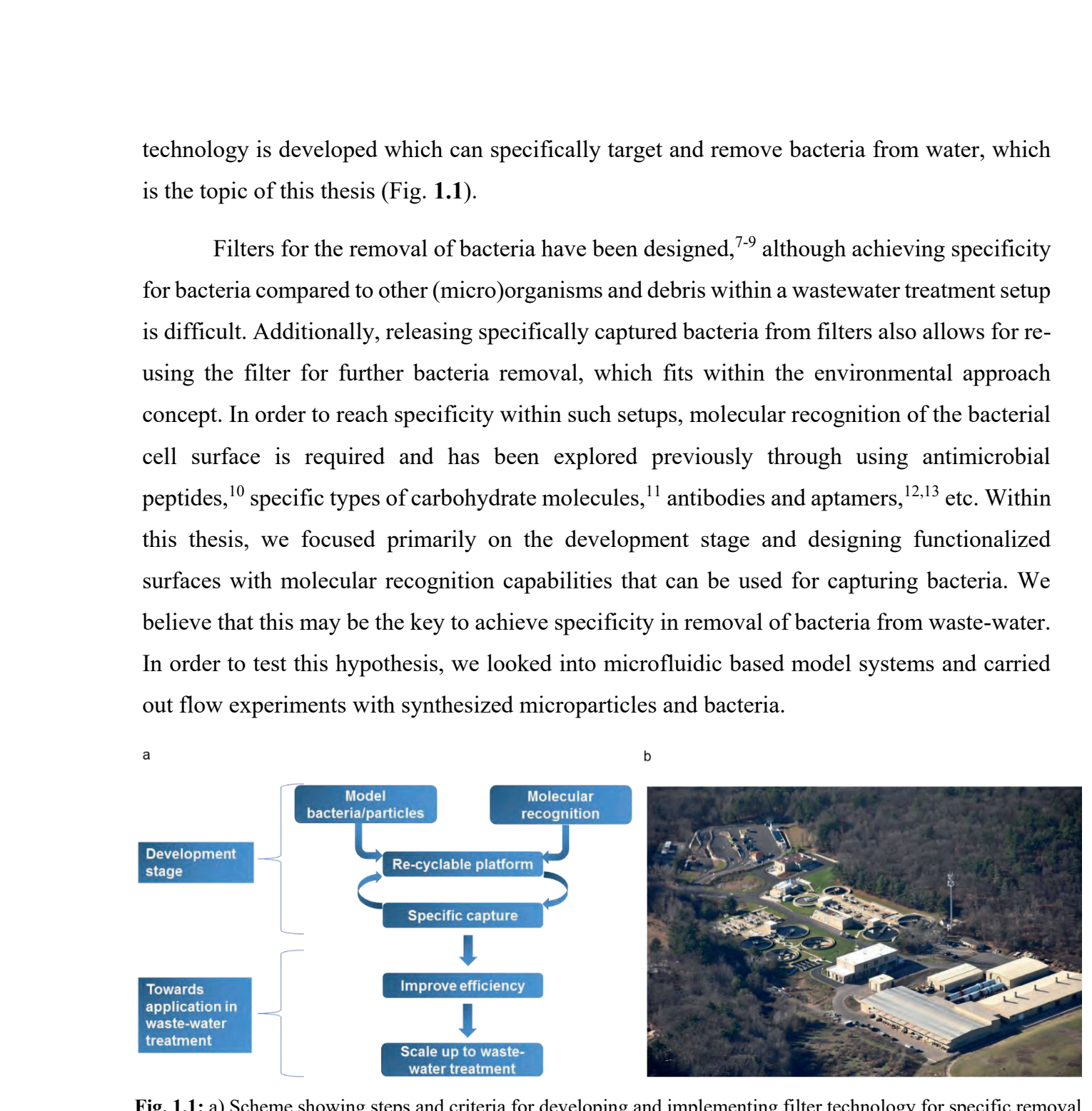

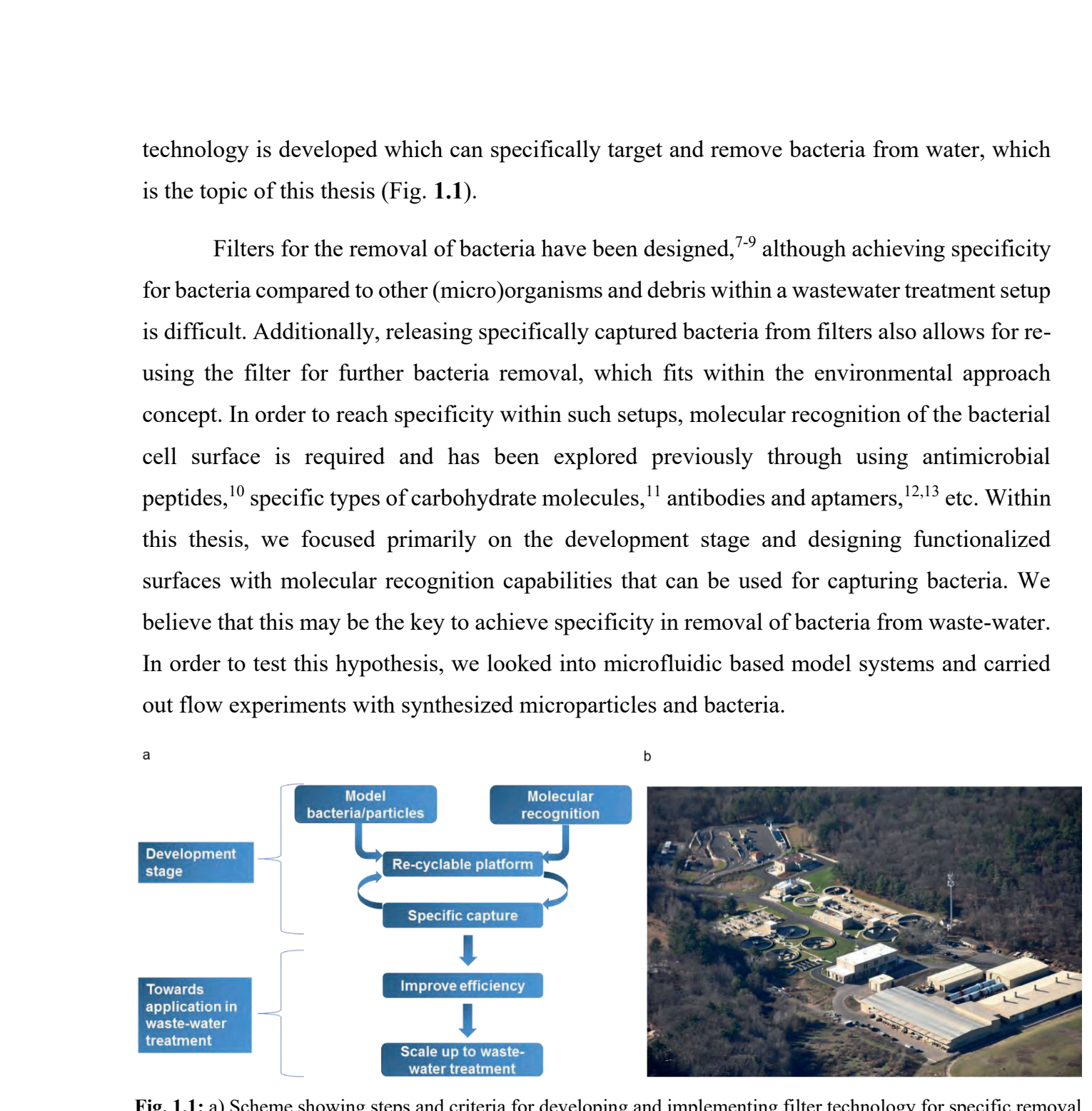

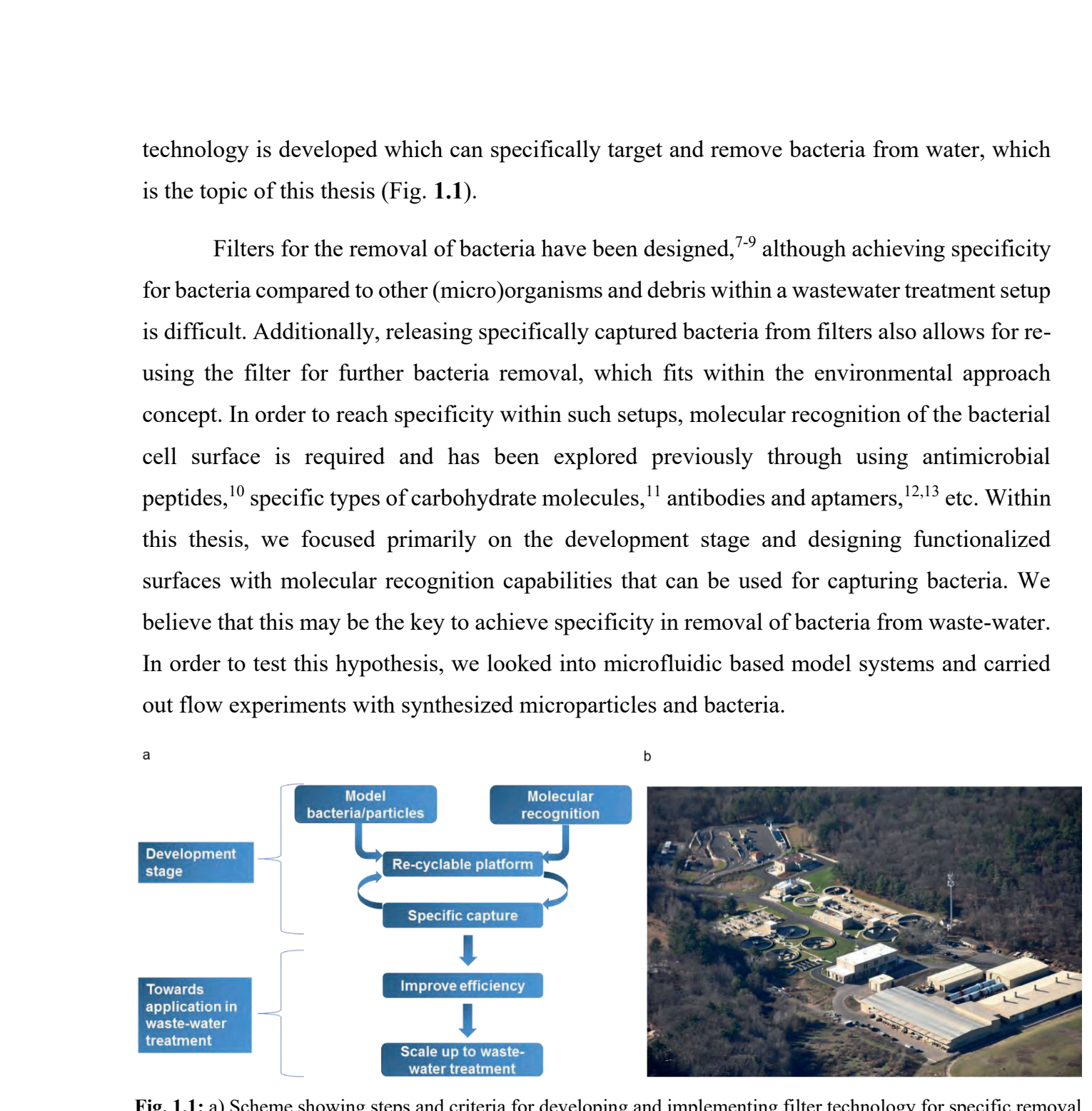

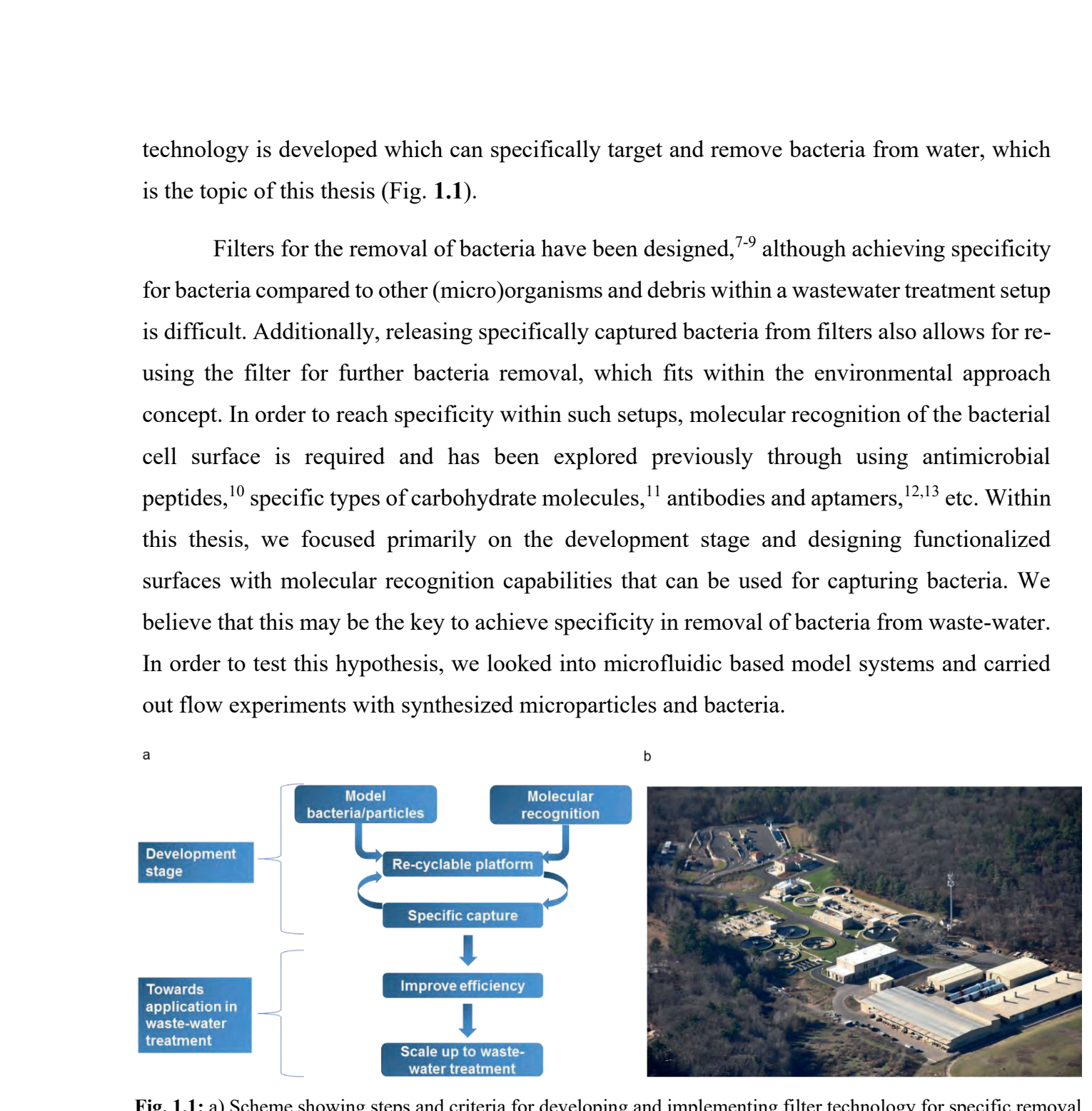

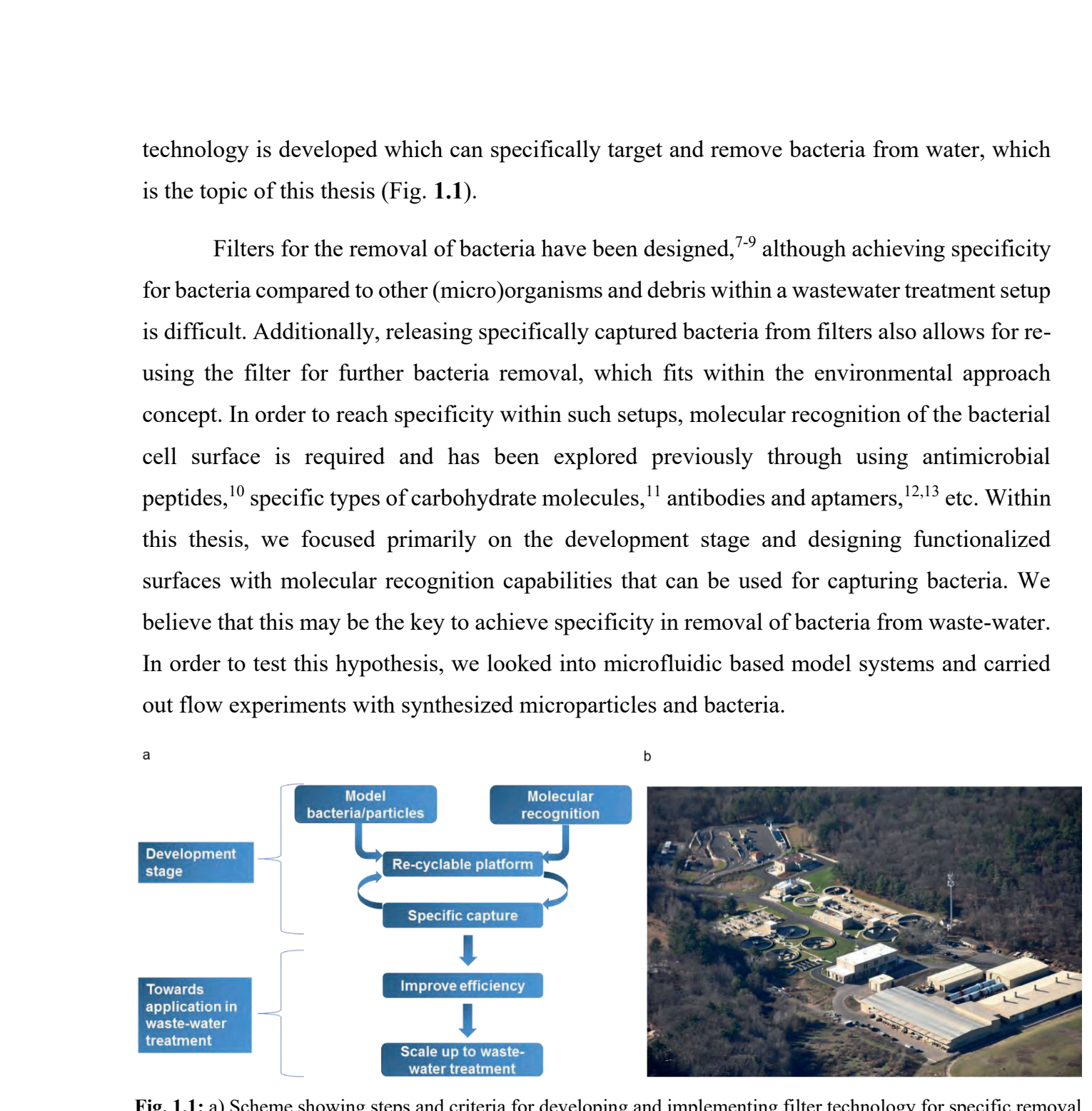

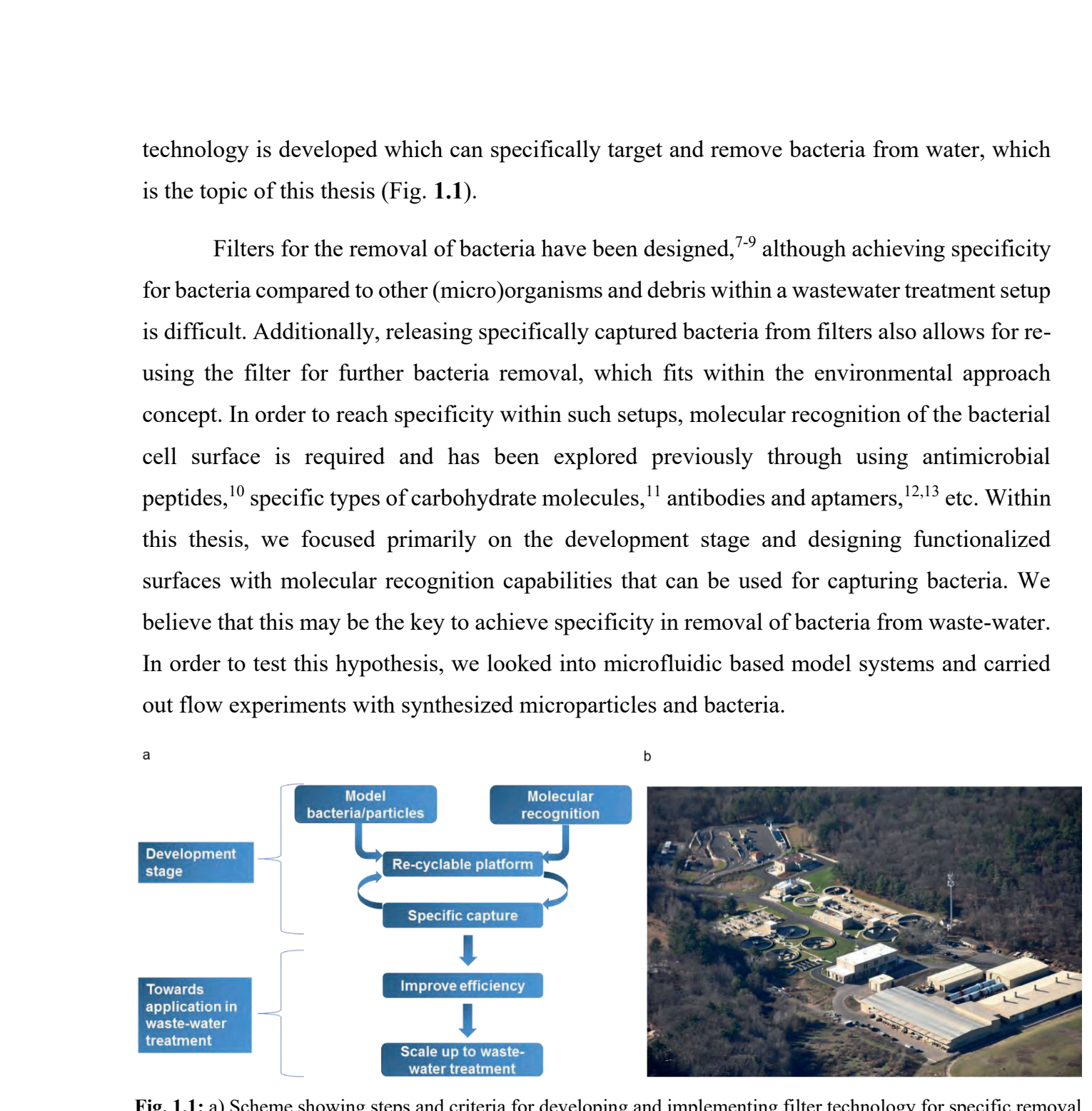

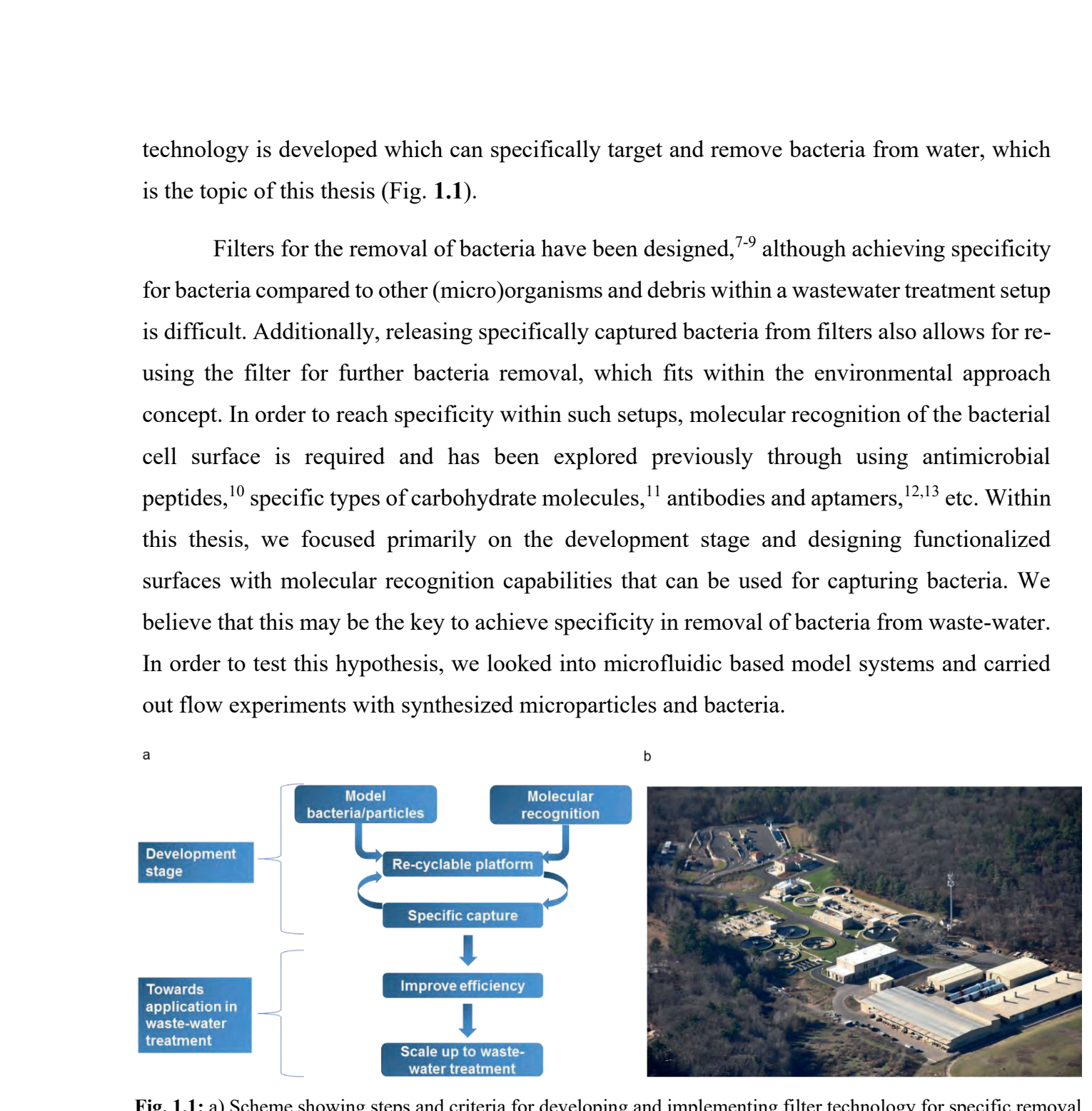

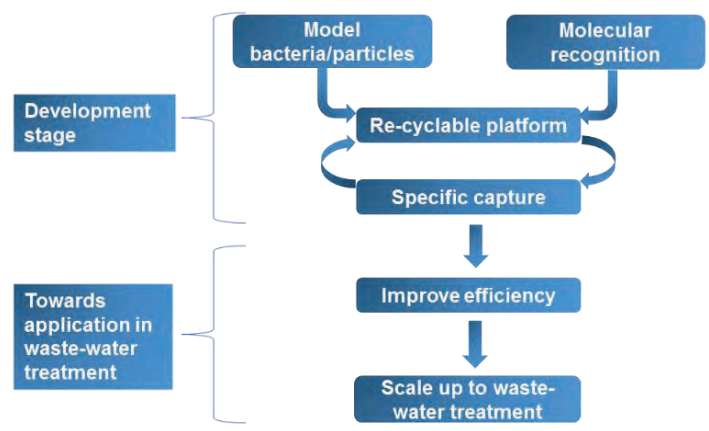
of bacteria from waste water. b) Picture of a waste-water treatment plant to showcase large scale of water treatment where the filter technology would be implemented.

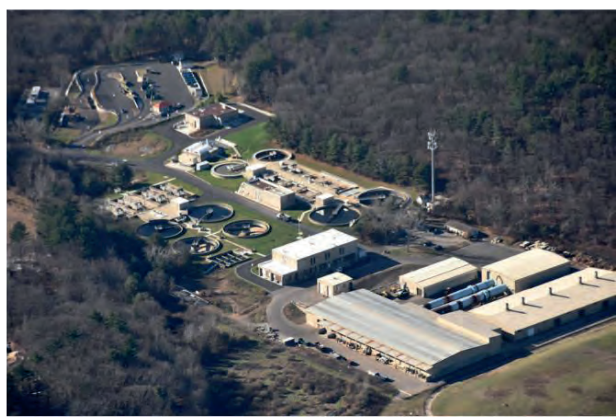

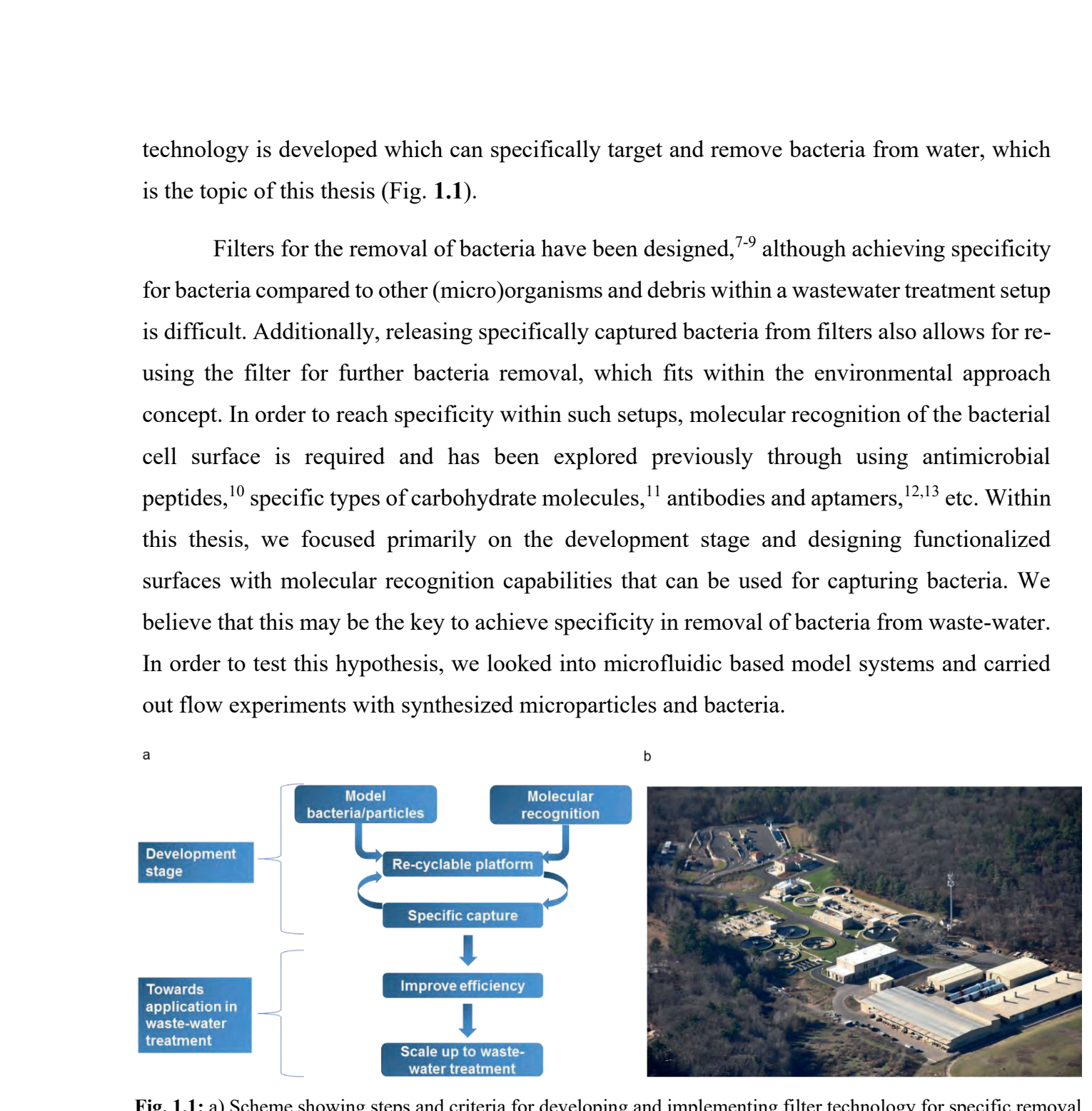

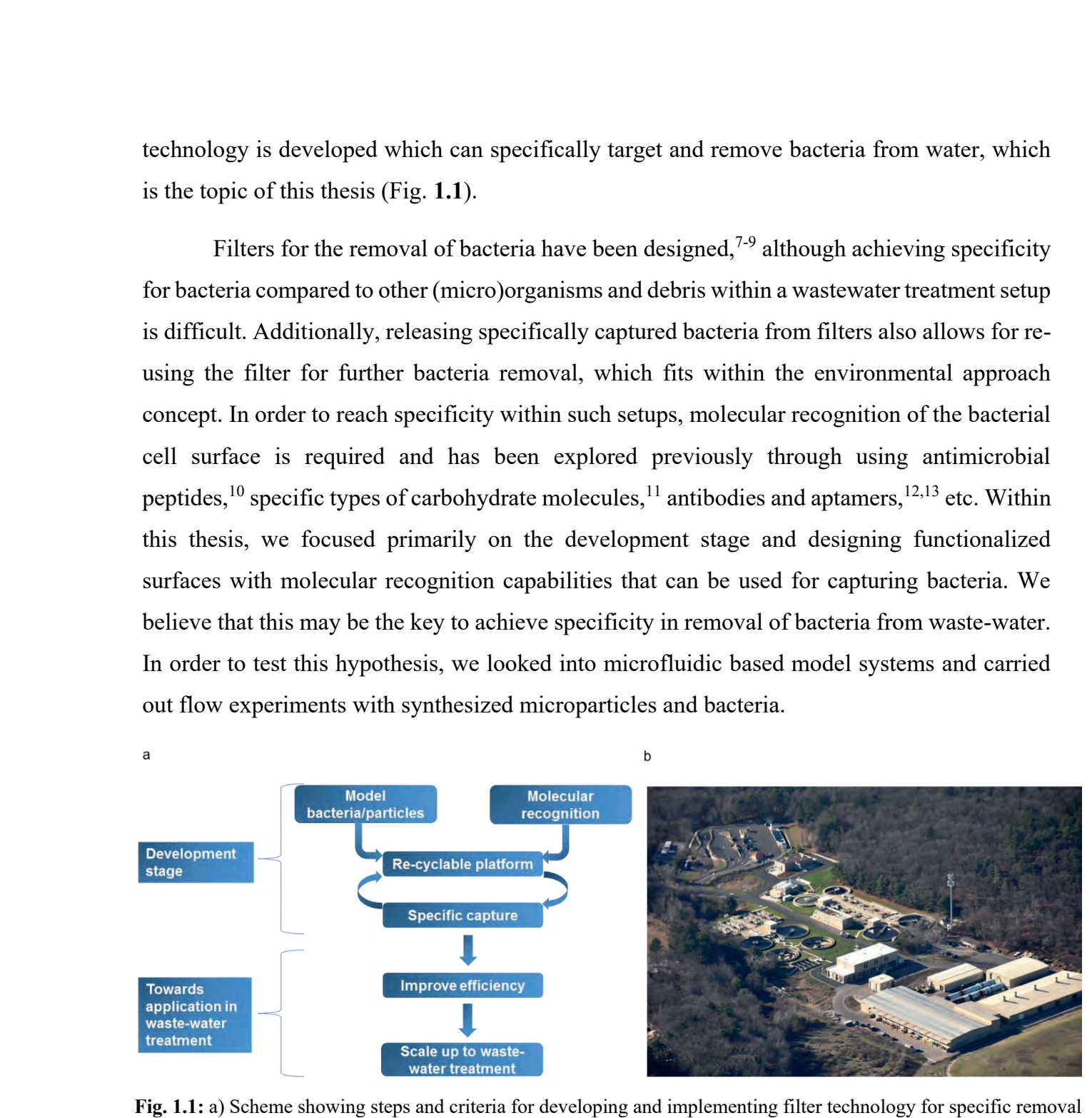

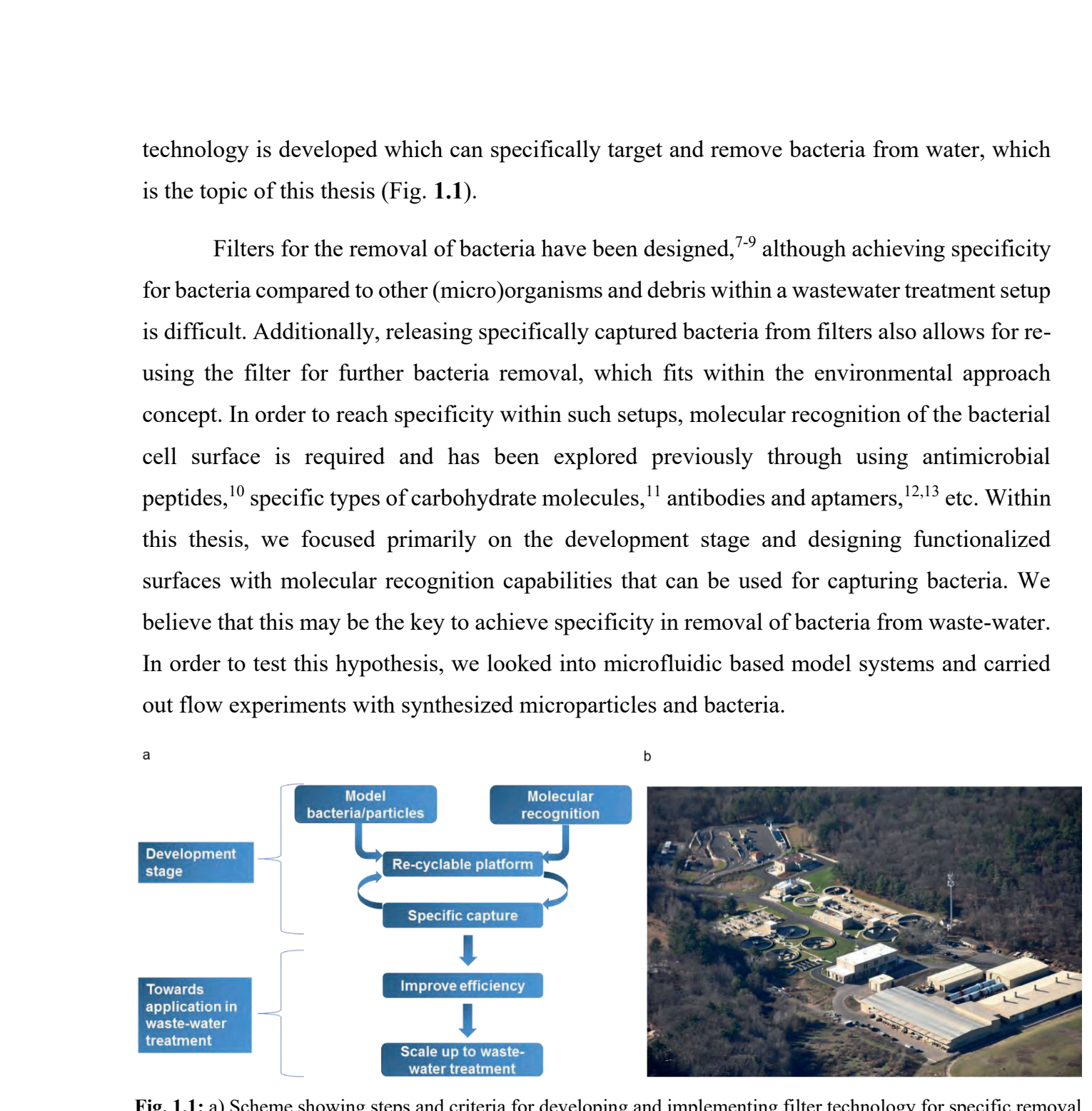




\subsection{Supramolecular platforms for pathogen removal}

Within the development of new technology to target and remove bacteria from water, we functionalized surfaces with molecules capable of supramolecular interactions to act as filters for capturing bacteria. The complexity of the bacterial cell surface is shown in Fig. 1.2 $(\mathbf{a}+\mathbf{b})$, which also shows the scale difference between components (nanometer) within the cell envelope and the actual size of the bacterial cell (micrometer). By using host-guest interactions, the cell surface of micrometer sized bacteria will be targeted by nanometer sized molecules.

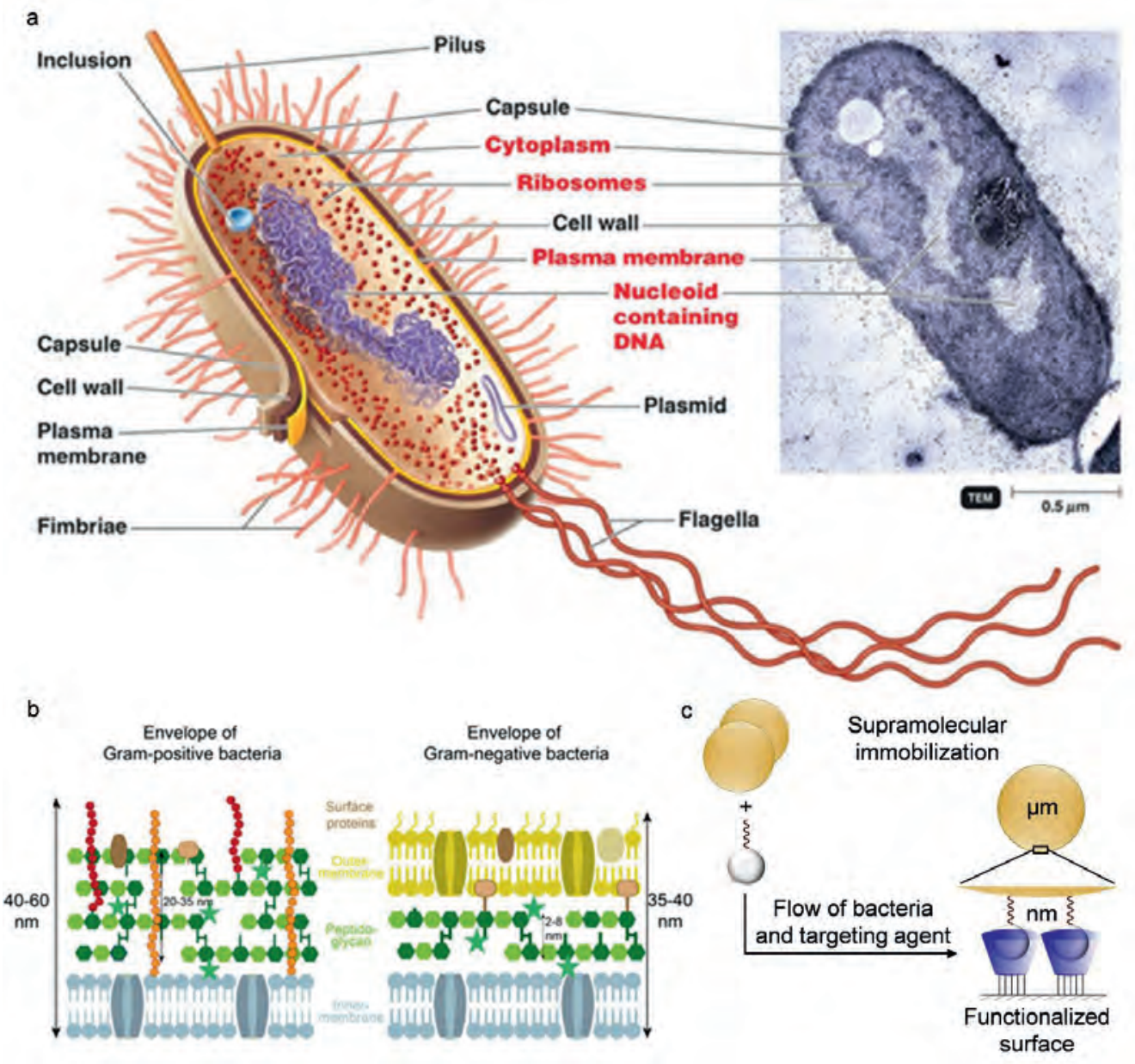

Fig. 1.2: a) Schematic representation of a bacterial cell showing the different components on the cell surface and within the cell, accompanied with a transmission electron microscopy (TEM) images of the cell. Figure taken from Tortora et al. ${ }^{14}$ b) Bacterial cell surfaces of Gram-positive and Gram-negative bacteria, also showing the different size ranges within the cell envelope. ${ }^{15} \mathrm{c}$ ) Concept of supramolecular immobilization of bacteria on functionalized surfaces using targeting agents. 
Modification of spherical objects such as millimeter-sized beads or porous structures will improve the capturing efficiency as there is a higher surface-to-volume ratio when the modified surface is in contact with an aqueous solution of molecules or particles. On the other hand, the use of flat rigid objects, such as glass surfaces, allows for easily studying the immobilization and release of molecules and particles like bacteria to the surface using microscopy. Therefore, considering the complex nature of bacteria and supramolecular targeting mechanisms, we fabricated supramolecular functionalized flat microscope glass slides to study on-flow immobilization processes (Fig. 1.2c). In this case, applying a flow of solution allows for approaching the application within wastewater treatment. Furthermore, the molecular functionalization on modified surfaces can be characterized, and incorporated in microfluidic devices for testing flow conditions.

\section{Glass surface functionalization and patterning}

The first step in developing a supramolecular glass platform to test for pathogen removal is basic surface functionalization and creation of molecular patterns to validate the modified surface. In the field of (bio)nanotechnology, surface functionalization with (self) assembled monolayers and patterning with soft lithography were spearheaded by G.M. Whitesides in the 90 's. ${ }^{16}$ Assembled monolayers (AM) are spontaneously formed ordered domains of organic molecules on surfaces through adsorption processes. The adsorption of molecules on glass surfaces has been carried out through 'silanization' with organosilanes, which are silicon based compounds similar to hydrocarbons. ${ }^{17}$ Popular organosilanes for glass surface modification include perfluoroctadecyltrichlorosilane (PFOTS), ${ }^{18}$ octadecyltrimethoxysilane (OTS), ${ }^{19} 3$ -

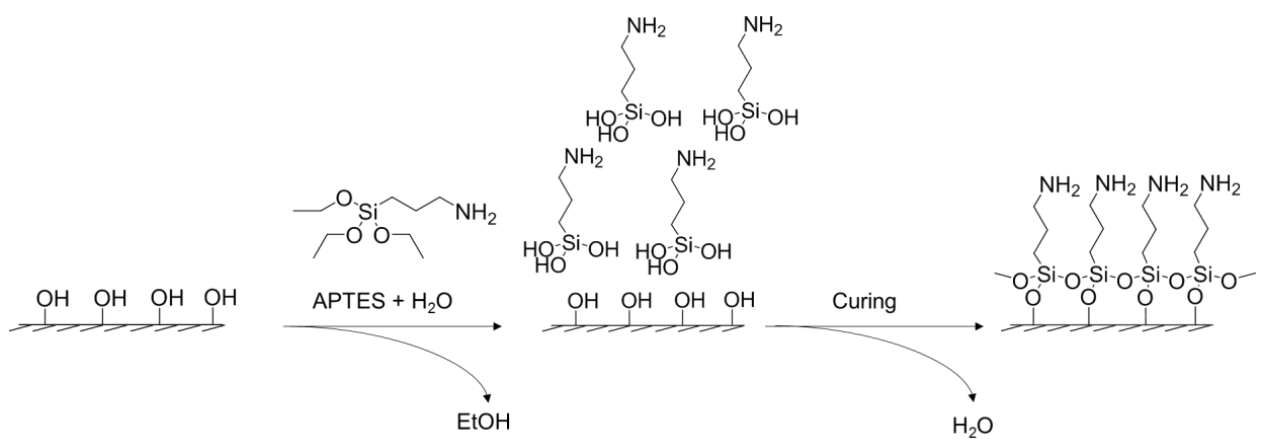

Fig. 1.3: Monolayer assembly of organosilanes to hydroxylated glass surfaces. In this case, APTES is used as an example for silanization. Trace amount of $\mathrm{H}_{2} \mathrm{O}$ is needed for forming intermediate silanol groups on the head end of the organosilane. The intermediate silanol groups on the organosilane then covalently react (curing) with the hydroxylated surface, regenerating water. 
aminopropyltriethoxysilane (APTES), ${ }^{20}$ and 3-mercaptopropyltriethoxysilane (MPTS). ${ }^{21}$ PFOTS and OTS are useful in particular for introducing anti-fouling capabilities on surfaces through their hydrophobic character, while APTES and MPTS, containing either amine or mercapto functional groups, allow for further surface functionalization steps through reactions with carboxylates, iso(thio)cyanates or thiol groups. APTES functionalized surfaces have been used for protein immobilization ${ }^{22}$, anchoring silver nanoparticles as antibiofilm surfaces ${ }^{23}$, and for binding thermoplastics to polydimethylsiloxane (PDMS). ${ }^{24}$ In general, silanization of glass surfaces requires a hydroxylated surface $(-\mathrm{OH})$ groups, a trace amount of $\mathrm{H}_{2} \mathrm{O}$, and specific reaction conditions depending on the organosilane. ${ }^{25}$ The trace amount of $\mathrm{H}_{2} \mathrm{O}$ forms intermediate silanol groups (head end) on the organosilane which in turn covalently reacts with the hydroxyl groups on the glass surface (Fig. 1.3). For methoxy- and ethoxysilanes, methanol and ethanol are released when the intermediate silanol groups are formed through hydrolysis, while for trichlorosilanes hydrochloric acid is formed. The tail end, which contains the functional group, subsequently becomes the dominant surface chemical species that influences the physical and chemical properties of the modified glass surface. We focused on APTES as organosilane for primary surface modification as the amino tail end group can be cross-linked to other amine containing (bio) molecules through using $p$-phenelyne diisothiocyanate (PDITC) as an intermediate.

Soft lithography entails a group of techniques in which (micro)structures are replicated in the form of a rubber-like, elastomeric stamp by using photomasks and molds. Whitesides and co-workers worked on improving the fabrication and validation of (bio)sensors on surfaces through including patterning of molecules on the surface by using a soft lithographic technique known as microcontact printing $(\mu \mathrm{CP}) .{ }^{26}$ By patterning a surface functionality with a polydimethylsiloxane (PDMS) stamp, the analyte immobilization is localized to the desired area of the surface which functions as an experimental control (Fig. 1.4). Subsequently, positive

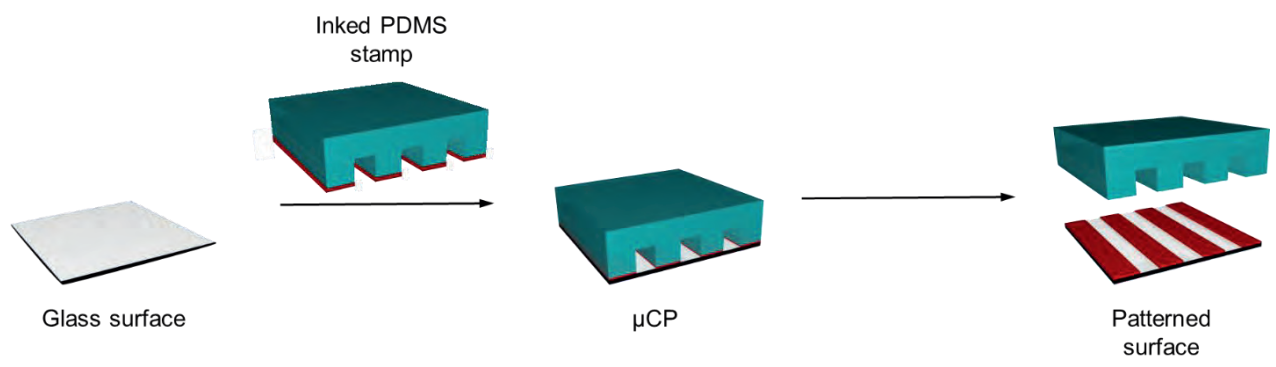

Fig. 1.4: Concept of patterning surfaces through $\mu \mathrm{CP}$ with PDMS stamps. A glass surface is subjected to conformal contact with a pre-inked PDMS stamp. The stamp is then removed to yield a patterned surface. 
and negative experimental controls can be placed in one field of view in order to qualify binding of molecules, particles or cells when viewing the surface using analytical techniques such as (fluorescence) microscopy. ${ }^{27}$

\section{Supramolecular interactions and host-guest chemistry}

Further modification of glass surfaces after silanization has been carried out with more complex (super)structures such as proteins, ${ }^{28}$ cucurbiturils, ${ }^{29}$ cyclodextrins, ${ }^{30}$ etc. These molecules can form supramolecular complexes through self-assembly with other molecules. Self-assembly is a type of selective, nature-inspired molecular recognition that functions similarly to the lock-and-key mechanism of enzymes and their constituent substrates. ${ }^{31}$ Supramolecular chemistry also allows for multivalent and reversible interactions, resulting in quick and specific immobilization of molecules or particles that can be followed by a triggered release. Multivalency here means that multiple recognition events of the same kind are occurring simultaneously between two entities, ${ }^{32}$ such as several ligands on one entity interacting with several receptors on the other. Since multivalency increases binding affinity it also ensures selectivity between binding pairs.

Within host-guest chemistry, the most well-known compound is cyclodextrin, which is a natural, non-toxic cyclic oligosaccharide shaped like a cone. Cyclodextrins have a hydrophilic external structure that consists of $(\alpha-1,4)$-linked $\alpha$-D-glucopyranose units and a hydrophobic internal cavity. The internal cavity can be used for encapsulating hydrophobic guest molecules through host-guest interactions (Fig. 1.5a). ${ }^{33}$ Host-guest chemistry is driven by hydrophobic and van der Waals interactions which allow hydrophobic (guest) molecules to form reversible inclusion complexes with the cyclodextrin (host) cavity (Fig. 1.5b). The three most popular
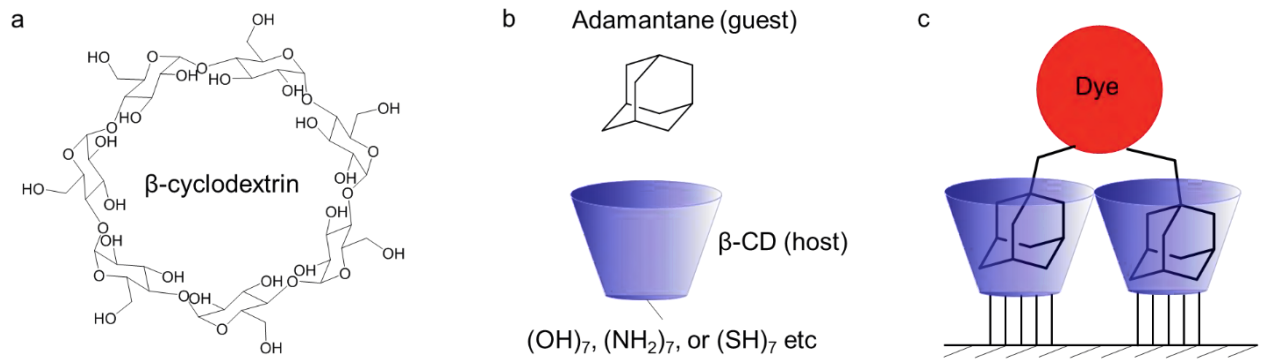

Fig. 1.5: a) Structure of naturally occurring form of $\beta-C D$. b) Truncated form of $\beta-C D$ (host) showing possibility of different functional groups on external structure and structure of adamantane (guest) which can form hostguest inclusion complexes with $\beta-\mathrm{CD}$ cavity. c) Multivalent host-guest interactions of $\beta-\mathrm{CD}$ functionalized surface with diadamantane functionalized fluorophores. 
naturally occurring cyclodextrins are $\alpha-, \beta-$, and $\gamma$-cyclodextrin which respectively increase in size and consist of six, seven and eight glucopyranose units. The larger the cyclodextrin, the bigger the hydrophobic cavity size for encapsulating larger guests. In order to accommodate the use of cyclodextrins for specific applications, substitution of hydroxyl groups on the external structure with e.g. amine or thiols groups has been carried out and these chemically modified cyclodextrins are also commercially available. For these reasons, cyclodextrin is used in a wide variety of applications within drug delivery, food technology, and the pharmaceutical industry. ${ }^{34,35}$ Surfaces have been functionalized with $\beta$-cyclodextrin $(\beta-\mathrm{CD})$ in order to capture molecules or nanoparticles modified with guest moieties such as adamantane, ${ }^{36-38}$ ferrocene, ${ }^{39}$ or azobenzene (Fig. 1.5c). ${ }^{40}$ The host-guest interactions with these systems all have their unique properties such as the strong interaction between adamantane- $\beta-C D$ and reversible binding with ferrocene- and azobenzene- $\beta$-CD through chemical oxidation or UV-light exposure.

Throughout this dissertation, the focus is on the fabrication of $\beta$-CD patterned surfaces as a platform for potential use within bacterial cell capturing. An important step for platform development is characterization of the modified surface, which can be done by visualizing and confirming $\beta$-CD patterns through using a guest-functionalized fluorophore. To this end, we used a diadamantane functionalized Cyanine 5 dye. ${ }^{41}$ This dye can be immobilized rapidly on the patterned $\beta-C D$ area through using divalent host-guest interactions with two $\beta-C D$ cavities. Moreover, the re-usability of these fabricated surfaces can be characterized owing to the reversible nature of the formed inclusion complexes.

\section{Fluorescence microscopy for characterizing surfaces and surface interactions}

Fluorescence microscopy, with either optical or confocal laser scanning microscope systems, was used through this thesis as the main tool for characterizing patterned, functionalized surfaces and studying interactions of surfaces with molecules, particles and bacteria. Fluorescent dyes are generally attached to surfaces for characterization purposes as they provide a strong emission of light which can be visualized using optical and confocal microscopes and respective cameras depending on the wavelength of emission. Even at monolayer amounts of fluorescent dye, the signal intensity is high enough to be detected when using microscopy. In our case, validating a $\beta$-CD modified surface through addition of a diadamantane functionalized dye proves accessibility of $\beta$-CD`s hydrophobic cavity and therefore availability for host-guest interactions. 
Cyanine and Rhodamine B dyes were used for characterizing functionalized surfaces and staining particles used in binding experiments with modified surfaces. Rhodamine B is soluble in aqueous and alcoholic solutions and Cyanine dyes can also exist in a sulfonated form in order to allow solubility in aqueous solutions, although their quantum yield is higher in organic solvents. Cyanine dyes are ideal for use within fluorescence microscopy as they have a high extinction coefficient ( 130 000-250 $\left.000 \mathrm{M}^{-1} \mathrm{~cm}^{-1}\right)$, are quite stable in physiological conditions, and have a fluorescence emission which covers a broad range of wavelengths (500$750 \mathrm{~nm}) .{ }^{42}$ The succinimidyl ester reactive groups can be conjugated to amino-group containing molecules to incorporate use within (bio)applications. The success of Cyanine dyes such as Cyanine 5 (Cy5) has been outlined by their use in medical applications, ${ }^{43,44}$ in which high brightness and resistance to photobleaching was paramount to successful analysis of labeled targets. Therefore, glass surface characterization was carried out with rhodamine B isothiocyanate staining for validation of amine groups on 3-aminopropyltriethoxysilane (APTES) and sulfonated Cyanine 5 functionalized to two adamantane molecules (Cy5-Ad2) was used for labeling pre-patterned $\beta-\mathrm{CD}$ lines on (chapters 2 and 3). Hydrophobic Cy3 and Cy5.5 for staining the styrene core in polystyrene particles (chapter 4) and standard Hoechst 33342 staining was used for visualization of bacteria used in chapter 5 . The structure of these dyes is shown in Fig. 1.6, along with approximate absorption and emission ranges of the dyes to take into account with epi-fluorescence and confocal microscopy. 


\section{Cy3}

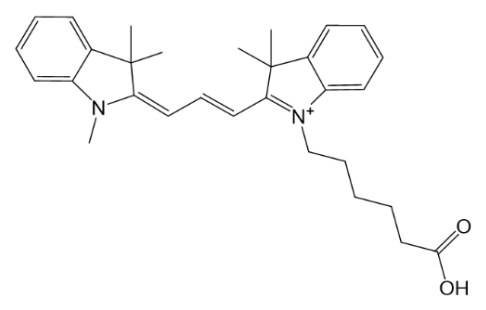

Cy5- $-\mathrm{Ad}_{2}$

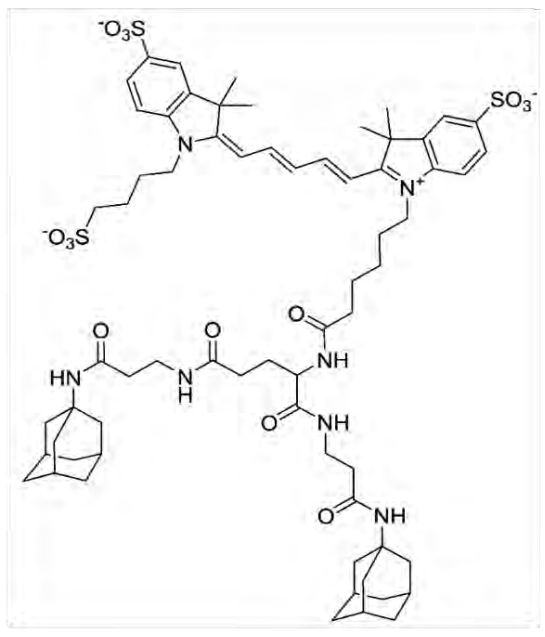

Hoechst 33342

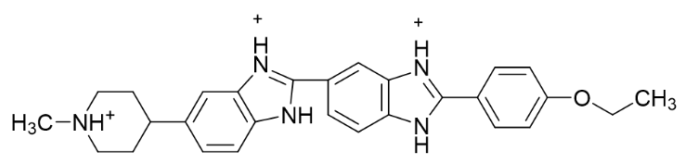

\section{RITC}<smiles></smiles>

\section{Cy5.5}

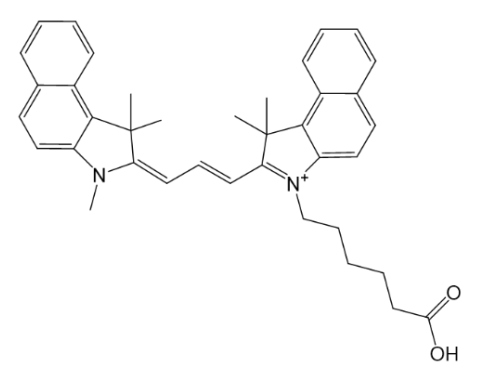

Hoechst $33342 \quad 360-400 \quad 405-800$

Cy3, RITC 541-551 565-605

$\mathrm{Cy}-\mathrm{Ad}_{2} \quad 590-650 \quad 662-738$

Cy5.5 $\quad 660-690 \quad 700-750$

Fig. 1.6: Structures of dye molecules Cyanine 3 (Cy3), rhodamine B isothiocyanate (RITC), Cyanine 5diadamantane (Cy5- $\left.\mathrm{Ad}_{2}\right)$, Cyanine 5.5 (Cy5.5), Hoechst 33342, and a table showing approximate excitation and emission ranges for fluorescence microscopy. 


\section{Incorporation of functionalized surfaces in microfluidic devices}

By applying a flow of bacteria over the functionalized surface, we approach the application of bacteria removal within waste-water treatment plants. However, such flow experiments are ideally carried out on small scale within the preliminary phase. Microfluidics is the method of choice for mimicking the practical situation, as it focuses on the behavior and control of fluids within a geometrically constrained area such as a flow channel, generally within the micrometer to millimeter size range. ${ }^{45-46}$ It has therefore emerged as an interdisciplinary topic in chemistry, biology, ${ }^{47}$ physics, ${ }^{48-49}$ engineering, ${ }^{50}$ biochemistry,${ }^{51}$ and nanotechnology. ${ }^{52}$ Re-sealable microfluidic flow cell systems from, for example, Micronit ${ }^{2}$, include an 'empty' glass slide and a gasket slide. The 'empty' glass slide can be functionalized to include the desired surface moiety/chemistry and the gasket slides contains the microchannel to determine the flow path of fluids. The two glass slides are pressed together when placed in the 'flow cell holder', which allows for incorporating inlet and outlet tubing.

Besides commercial microfluidic systems, fabrication of lab-built microfluidic devices is also a highly explored topic for lab-on-a-chip applications. ${ }^{53}$ This concept allows for more versatility within microfluidic device fabrication, such as varying microfluidic channel size and amount of channels running over one surface, according to research needs. Within lab-built microfluidic devices, the most popular are based on PDMS/glass hybrid microfluidic systems which are fabricated through replica molding and plasma etching ideated by Whitesides and co-workers. ${ }^{54-55}$ The slab of PDMS/PDMS replica, constructed via replica molding from masters containing (micro)features, forms microchannels when attached to the glass. 
a

Re-sealable flow cell holder

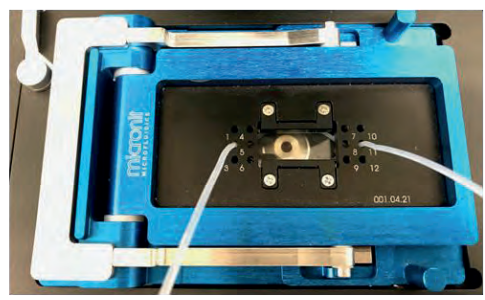

C b PDMS/glass microfluidic device

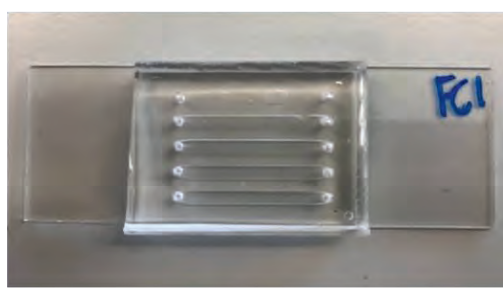

Modified surface in

microfluidic device

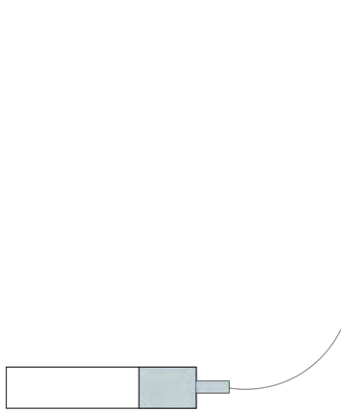

Pump and syringe with particles/bacteria

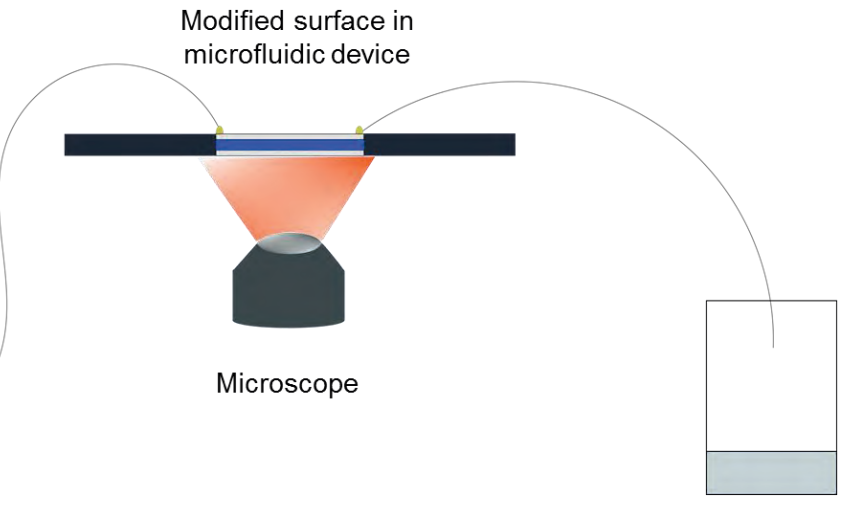

Waste beaker

Fig. 1.7: Photographs showing a) re-sealable microfluidic flow cell device incorporated in flow cell holder and b) labbuilt microfluidic device fabricated through replica molding. c) Flow setup over microscope which includes syringe pump for controlling flow rate of solution. 


\subsection{Experimental setup}

Through using the microfluidic setup with modified surfaces as an analytical toolbox, the specific immobilization of bacteria mediated by molecular recognition mechanisms can be ascertained (Fig. 1.7). Supramolecular functionality on surfaces provides a versatile platform for incorporating targeting agents modified with guest molecules such as adamantane, azobenzene, ferrocene, etc. These targeting agents, functioning as an intermediary between planar surface and bacteria, can then facilitate the immobilization of bacteria to $\beta$-CD patterns on the surface. Analyte binding defines the final utility of the microfluidic sensor, as retention of the bacteria on $\beta-C D$ patterns within the microfluidic device depends on successful molecular recognition which can then be analyzed using microscopy. As a first step, however, the feasibility of using supramolecular host-guest chemistry for immobilizing micrometer sized entities such as bacteria was validated by using model particles.

\section{Polystyrene model particles}

Synthesized particles can be used to mimic biological systems such as viruses or bacteria as it allows for studying certain types of interactions in a more controlled manner. For example, hemagluttinin nanoparticles have been used as model particles for an influenza virus in order to study the nanoparticle's immobilization to surfaces. ${ }^{56}$ The use of this model system led to
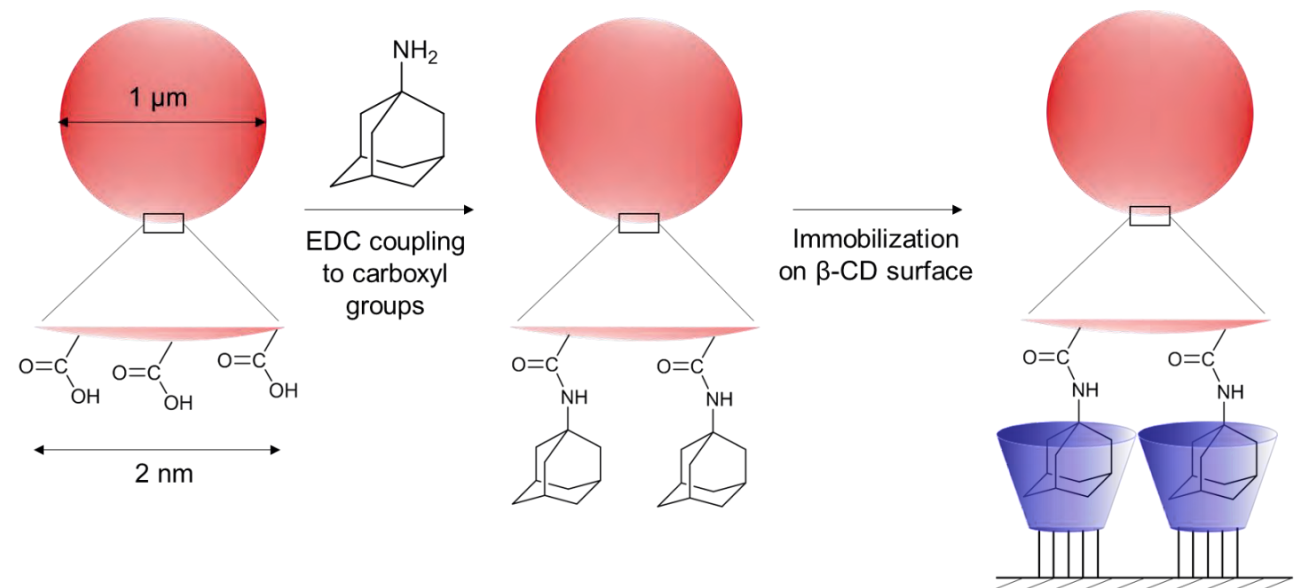

Fig. 1.8: Amide coupling of adamantane amine to carboxyl group on model microparticles mediated by 1-ethyl-3(3-dimethylaminopropyl)carbodiimide (EDC) and subsequent addition over $\beta$-CD modified surface for particle immobilization through host-guest interactions. 
the conclusion that weak, multivalent interactions were involved in the particle's immobilization to the surface and are important for the virus' biological functioning. However, supramolecular functionalized surfaces have mostly been used for host-guest interactions of analytes within the nano-size range. Scaling up nanometer scale host-guest interactions toward capturing bacteria could pose a challenge in terms of larger drag forces acting on micrometer sized bacteria. The effect of this size difference can be roughly compared to an ant capturing a car. Within this situation, multivalency will be key to providing a high enough binding affinity to overcome drag forces acting on micro-sized entities, especially when a flow of solution is applied. Experimenting with model particles therefore provides information on the feasibility of capturing micro-sized entities with nano-sized supermolecules through multivalent hostguest interactions.

Polystyrene (PS) particles are eligible model particles for bacteria as they can also be synthesized at $1 \mu \mathrm{m}$ size. ${ }^{57}$ They are formed through dispersion polymerization from styrene monomers to form particles which can contain a fluorophore and a specific functionality on the particle surface such as carboxyl, amine, or hydroxyl groups. Inclusion of a fluorophore within the polystyrene core of the particle also allows for facile discrimination on the fluorescence microscope when using different species of PS particles in solution. Therefore, they can also be tailored to meet specific requirements for the model system, such as including adamantane guest molecules on the particle surface which can be targeted by $\beta$-CD functionalized platforms (Fig. 1.8).

\section{Model bacteria}

In this thesis, two model bacteria were used for experiments: Staphylococcus aureus (S. aureus) and Escherichia coli (E. coli). This selection of bacteria was made based on their pathogenic effect on society and their different inherent nature e.g. gram-positive vs gramnegative. We hypothesize that these bacteria can be targeted and captured on $\beta-C D$ functionalized surfaces through using host-guest interactions.

Staphylococcus aureus (S. aureus) is a gram-positive, round shaped bacterium discovered by Alexander Ogston in 1881. It is a food born pathogen, commonly found in protein-rich food, such as meat and dairy products. ${ }^{58}$ Although approximately $25-35 \%$ of people are actually long-term carrier of the bacterium, ${ }^{59}$ S. aureus can cause diseases, and infections were fatal prior to the 1940's before the discovery of penicillin by Alexander Fleming. Present- 
day, the rise of methicillin resistant $S$. aureus (MRSA) from hospital-associated infections is becoming increasingly difficult to treat. ${ }^{60}$ MRSA also spreads through livestock farming, where antibiotic resistance is increasing through overuse of $\beta$-lactam antibiotics. The removal of this type of bacteria from environmental water reservoirs is therefore of importance.

The second bacterium that we used within bacterial binding experiments is Escherichia coli (E. coli), which is gram-negative, rod shaped bacterium discovered by Theodor Escherich in 1885. It is commonly found as a harmless strain in the intestines of human beings where it functions symbiotically and prevents colonization by invading microbes. However, it is known that pathogenic strains of E. coli cause issues such as food poisoning, meningitis, and gastroenteritis. ${ }^{61}$ Within the food industry, antibiotic strains of $E$. coli such as extended spectrum $\beta$-lactamase (ESBL) producing $E$. coli has also received attention recently. ${ }^{62}$

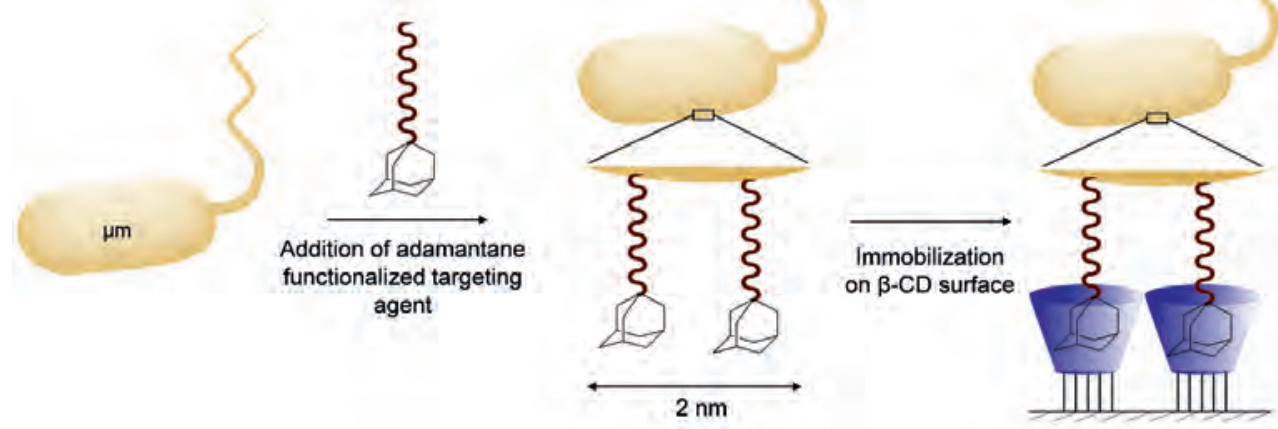

Fig. 1.9: Functionalization of bacteria cell surface with adamantane functionalized targeting agent and subsequent immobilization on $\beta-C D$ functionalized surface through host-guest interactions. 


\subsection{Motivation and outline of this thesis}

Developing technology for specific removal of pathogenic bacteria from waste water streams is a challenging goal and several important criteria can be set as milestones. These milestones include:

- capturing bacteria at a high enough binding affinity for use with high flow rates of water

- reversibility of binding mechanisms so bacteria can be released in an isolated environment and exterminated

- re-usability of the developed surfaces to decrease costs and environmental impact

- achieving specificity so that only pathogenic bacteria are targeted and immobilized

Here, the focus was placed on chemically modifying surfaces through covalent functionalization and using microfluidics as a model system for waste-water flow. This aided in qualitatively determining the added benefit of using specific targeting molecules for selective molecular recognition of model particles and bacteria. Therefore, the first part of the thesis is about surface functionalization and patterning on glass surfaces, followed by incorporation within microfluidic devices. Subsequently, the functionalized surfaces within microfluidic devices were tested with model particles and bacteria. For these experiments, the binding was characterized through varying flow rate of the solution, changing targeting ligand density on the particle surface, and for reversibility.

In Chapter 2, an alternative method to microcontact printing ( $\mu \mathrm{CP}$ ) for creating patterns of covalently functionalized monolayers on glass surfaces is presented. Having molecular patterns on surfaces for sensing applications is important as an experimental control during microscope analysis for the detection of (bio) analytes, such as molecules, particles and bacteria. In a process called plasma microcontact patterning $(\mathrm{P} \mu \mathrm{CP})$, molecular patterns are obtained by using plasma etching in synergy with a patterned PDMS stamp placed on top of a functionalized surface, which removes covalently bound molecules at non-protected areas. Creating patterns through $\mathrm{P} \mu \mathrm{CP}$ was studied at different steps in a surface functionalization protocol for fabrication of $\beta$-CD monolayers, and the patterns were visualized through addition of Cy5-Ad2. We also studied the effect of changing the orientation of the PDMS stamp multiple times during the $\mathrm{P} \mu \mathrm{CP}$ step, in order to remove more of the surface functionalization, and then subjecting the surface to a solution of $\mathrm{Cy} 5-\mathrm{Ad}_{2}$. 
In Chapter 3, creating covalently functionalized patterns on glass surfaces by $\mathrm{P} \mu \mathrm{CP}$ are combined with fabrication of PDMS/glass hybrid microfluidic devices through conventional replica molding. By using plasma treatment on surfaces, silanol groups are generated on nonprotected areas during $\mathrm{P} \mu \mathrm{CP}$. This is the same for plasma treated PDMS surfaces and when both 'activated' glass and PDMS are brought in contact with each other, they covalently attach to each other through 'plasma bonding'. Therefore, we investigated the attachment of patterned $\beta-C D$ functionalized surfaces to PDMS replicas containing microchannel features, and characterize the resulting microfluidic devices for chemical reactivity and re-usability with Cy5-Ad 2 dye.

In Chapter 4, the on-flow immobilization of adamantane functionalized polystyrene particles to $\beta-C D$ patterns is tested as a model system for bacterial cell immobilization. In this case, conventional $\mu \mathrm{CP}$ was used to pattern the surface with $\beta-\mathrm{CD}$ and a commercially available re-sealable flow cell system is used as microfluidic device for flow experiments. The model system allows for experimenting on several important parameters concerning bacterial cell immobilization for waste-water treatment, such as flow rate variation, targeting molecule density (adamantane loading on PS particles), and re-usability of fabricated surfaces. Furthermore, the obtained results regarding flow rate variation are compared with Comsol simulations and explained in terms of water physics.

In Chapter 5, the microfluidic devices with $\beta$-CD patterns fabricated in Chapter 3 are used for bacterial cell capturing experiments with $S$. aureus and E. coli model bacteria. The bacterial cells are targeted through an antimicrobial peptide isolated from ubiquitin, $\mathrm{UBI}_{29-41},{ }^{10}$ which is functionalized with two adamantane moieties (UBI-Ad $)_{2}$. We studied the effect of incubating $\mathrm{UBI}-\mathrm{Ad}_{2}$ with bacterial cells before flow experiments over $\beta-\mathrm{CD}$ patterned microfluidic devices, or first incubating $\beta-\mathrm{CD}$ patterns on surfaces with UBI- $\mathrm{Ad}_{2}$ prior to bacteria flow. Additionally, the effect of changing the flow rate and addition of unmodified bacteria over $\beta$-CD patterns on surfaces was also studied.

In Chapter 6, a general discussion on the use of supramolecular host-guest interactions for removal of pathogenic bacteria from waste-water is provided. The experimental findings, implications, limitations and opportunities originating from the four experimental chapters are discussed. For approaching the application in waste-water treatment, suggestions for follow up projects and research are given and also a conclusion is provided. 


\subsection{References}

1. Whitman, W. B.; Coleman, D. C.; Wiebe, W. J. Prokaryotes: the unseen majority. Proceedings of the National Academy of Sciences of the United States of America 1998, 95 (12), 6578-6583.

2. Hogan, C. M. Bacteria. Encyclopedia of Earth. Washington DC: National Council for Science and the Environment 2010.

3. Moore, Simon J.; Warren, Martin J. The anaerobic biosynthesis of vitamin B12. Biochemical Society Transactions 2012, 40 (3), 581.

4. Floyd, K.; Glaziou, P.; Zumla, A.; Raviglione, M. The global tuberculosis epidemic and progress in care, prevention, and research: an overview in year 3 of the End TB era. The Lancet Respiratory Medicine 2018, 6 (4), 299-314.

5. Bennett, J. W.; Chung, K. T. Alexander Fleming and the discovery of penicillin. Advanced Applied Microbiology 2001, 49, 163-184.

6. Neu, H. C. The Crisis in Antibiotic Resistance. Science 1992, 257 (5073), 1064.

7. MacRae, I. C.; Evans, S. K. Removal of bacteria from water by adsorption to magnetite. Water Research 1984, 18 (11), 1377-1380.

8. Torkelson, A. A.; da Silva, A. K.; Love, D. C.; Kim, J. Y.; Alper, J. P.; Coox, B.; Dahm, J.; Kozodoy, P.; Maboudian, R.; Nelson, K. L. Investigation of quaternary ammonium silanecoated sand filter for the removal of bacteria and viruses from drinking water. Journal of Applied Microbiology 2012, 113 (5), 1196-1207.

9. Shtarker-Sasi, A.; Castro-Sowinski, S.; Matan, O.; Kagan, T.; Nir, S.; Okon, Y.; Nasser, A. M. Removal of bacteria and Cryptosporidium from water by micelle-montmorillonite complexes. Desalination and Water Treatment 2013, 51 (40-42), 7672-7680.

10. Lupetti, A.; Welling, M. M.; Pauwels, E. K. J.; Nibbering, P. H. Radiolabelled antimicrobial peptides for infection detection. The Lancet Infectious Diseases 2003, 3 (4), 223 229.

11. Lengeler, J. W.; Jahreis, K. Bacterial PEP-dependent carbohydrate: phosphotransferase systems couple sensing and global control mechanisms. In Bacterial Sensing and Signaling, Karger Publishers: 2009; Vol. 16, pp 65-87.

12. Bertozzi, C. R.; Bednarski, M. D. Antibody targeting to bacterial cells using receptorspecific ligands. Journal of the American Chemical Society 1992, 114 (6), 2242-2245.

13. Lee, Y. J.; Han, S. R.; Maeng, J.-S.; Cho, Y.-J.; Lee, S.-W. In vitro selection of Escherichia coli O157:H7-specific RNA aptamer. Biochemical and Biophysical Research Communications 2012, 417 (1), 414-420.

14. Tortora, G.; Funke, B.; Case, C. Principles of disease and epidemiology. Microbiology an introduction. 8th ed. San Francisco: Pearson Education Inc 2004, 408-431.

15. Jean, N., Bougault, C., Simorre, J.P., Glycopedia. The Structure of Bacterial Cell wall. https://glycopedia.eu/e-chapters/the-structure-of-bacterial-cell/article/introduction (Accessed Oct 9, 2019).

16. Kumar, A.; Biebuyck, H. A.; Whitesides, G. M. Patterning Self-Assembled Monolayers: Applications in Materials Science. Langmuir 1994, 10 (5), 1498-1511.

17. Dawood, M. 4 - Durability of steel components strengthened with fiber-reinforced polymer (FRP) composites. In Rehabilitation of Metallic Civil Infrastructure Using Fiber Reinforced Polymer (FRP) Composites, Karbhari, V. M., Ed. Woodhead Publishing: 2014; pp 96-114.

18. Psarski, M.; Celichowski, G.; Bystrzycka, E.; Pawlak, D.; Grobelny, J.; Cichomski, M. Vapor phase deposition of fluoroalkyl trichlorosilanes on silicon and glass: Influence of 
deposition conditions and chain length on wettability and adhesion forces. Materials Chemistry and Physics 2018, 204, 305-314.

19. Huang, J.; Liu, X.; Qiu, X.; Xie, L.; Yan, B.; Wang, X.; Huang, Q.; Zeng, H. Octadecyltrichlorosilane Deposition on Mica Surfaces: Insights into the Interface Interaction Mechanism. The Journal of Physical Chemistry B 2017, 121 (14), 3151-3161.

20. Peramo, A.; Albritton, A.; Matthews, G. Deposition of Patterned Glycosaminoglycans on Silanized Glass Surfaces. Langmuir 2006, 22 (7), 3228-3234.

21. Chen, J.-J.; Struk, K. N.; Brennan, A. B. Surface Modification of Silicate Glass Using 3-(Mercaptopropyl)trimethoxysilane for Thiol-Ene Polymerization. Langmuir 2011, 27 (22), 13754-13761.

22. Qin, M.; Hou, S.; Wang, L.; Feng, X.; Wang, R.; Yang, Y.; Wang, C.; Yu, L.; Shao, B.; Qiao, M. Two methods for glass surface modification and their application in protein immobilization. Colloids and Surfaces B: Biointerfaces 2007, 60 (2), 243-249.

23. Taglietti, A.; Arciola, C. R.; D'Agostino, A.; Dacarro, G.; Montanaro, L.; Campoccia, D.; Cucca, L.; Vercellino, M.; Poggi, A.; Pallavicini, P.; Visai, L. Antibiofilm activity of a monolayer of silver nanoparticles anchored to an amino-silanized glass surface. Biomaterials 2014, 35 (6), 1779-1788.

24. Sunkara, V.; Park, D.-K.; Hwang, H.; Chantiwas, R.; Soper, S. A.; Cho, Y.-K. Simple room temperature bonding of thermoplastics and poly(dimethylsiloxane). Lab on a Chip 2011, $11(5), 962-965$.

25. Glass, N. R.; Tjeung, R.; Chan, P.; Yeo, L. Y.; Friend, J. R. Organosilane deposition for microfluidic applications. Biomicrofluidics 2011, 5 (3), 36501-365017.

26. Xia, Y.; Whitesides, G. M. Soft Lithography. Angewandte Chemie International Edition 1998, 37 (5), 550-575.

27. Mrksich, M.; Chen, C. S.; Xia, Y.; Dike, L. E.; Ingber, D. E.; Whitesides, G. M. Controlling cell attachment on contoured surfaces with self-assembled monolayers of alkanethiolates on gold. Proceedings of the National Academy of Sciences of the United States of America 1996, 93 (20), 10775-10778.

28. Chen, Y.-X.; Triola, G.; Waldmann, H. Bioorthogonal Chemistry for Site-Specific Labeling and Surface Immobilization of Proteins. Accounts of Chemical Research 2011, 44 (9), 762-773.

29. Sankaran, S.; van Weerd, J.; Voskuhl, J.; Karperien, M.; Jonkheijm, P. Photoresponsive Cucurbit[8]uril-Mediated Adhesion of Bacteria on Supported Lipid Bilayers. Small 2015, 11 (46), 6187-6196.

30. Onclin, S.; Mulder, A.; Huskens, J.; Ravoo, B. J.; Reinhoudt, D. N. Molecular printboards: monolayers of beta-cyclodextrins on silicon oxide surfaces. Langmuir 2004, 20 (13), 5460-5466.

31. Mammen, M.; Choi, S.-K.; Whitesides, G. M. Polyvalent Interactions in Biological Systems: Implications for Design and Use of Multivalent Ligands and Inhibitors. Angewandte Chemie International Edition 1998, 37 (20), 2754-2794.

32. Krishnamurthy, V. M.; Estroff, L. A.; Whitesides, G. M. Multivalency in ligand design. Fragment-based Approaches in Drug Discovery 2006, 34, 11-53.

33. Szejtli, J. Introduction and General Overview of Cyclodextrin Chemistry. Chemical Reviews 1998, 98 (5), 1743-1754.

34. Loftsson, T.; Brewster, M. E. Pharmaceutical Applications of Cyclodextrins. 1. Drug Solubilization and Stabilization. Journal of Pharmaceutical Sciences 1996, 85 (10), 1017-1025.

35. Park, C.; Kim, H.; Kim, S.; Kim, C. Enzyme Responsive Nanocontainers with Cyclodextrin Gatekeepers and Synergistic Effects in Release of Guests. Journal of the American Chemical Society 2009, 131 (46), 16614-16615. 
36. Harrison, J. C.; Eftink, M. R. Cyclodextrin-Adamantanecarboxylate Inclusion Complexes: A Model System for the Hydrophobic Effect. Biopolymers: Original Research on Biomolecules 1982, 21 (6), 1153-1166.

37. Gonzalez-Campo, A.; Hsu, S. H.; Puig, L.; Huskens, J.; Reinhoudt, D. N.; Velders, A. H. Orthogonal covalent and noncovalent functionalization of cyclodextrin-alkyne patterned surfaces. Journal of the American Chemical Society 2010, 132 (33), 11434-11436.

38. Dorokhin, D.; Hsu, S.-H.; Tomczak, N.; Reinhoudt, D. N.; Huskens, J.; Velders, A. H.; Vancso, G. J. Fabrication and Luminescence of Designer Surface Patterns with $\beta$-Cyclodextrin Functionalized Quantum Dots via Multivalent Supramolecular Coupling. ACS Nano 2010, 4 (1), 137-142.

39. de laRica, R.; Fratila, R. M.; Szarpak, A.; Huskens, J.; Velders, A. H. Multivalent Nanoparticle Networks as Ultrasensitive Enzyme Sensors. Angewandte Chemie International Edition 2011, 50 (25), 5704-5707.

40. Voskuhl, J.; Sankaran, S.; Jonkheijm, P. Optical control over bioactive ligands at supramolecular surfaces. Chemical Communications 2014, 50 (96), 15144-15147.

41. Rood, M. T. M.; Spa, S. J.; Welling, M. M.; ten Hove, J. B.; van Willigen, D. M.; Buckle, T.; Velders, A. H.; van Leeuwen, F. W. B. Obtaining control of cell surface functionalizations via Pre-targeting and Supramolecular host guest interactions. Scientific Reports 2017, 7, 39908.

42. Mujumdar, R. B.; Ernst, L. A.; Mujumdar, S. R.; Lewis, C. J.; Waggoner, A. S. Cyanine dye labeling reagents: Sulfoindocyanine succinimidyl esters. Bioconjugate Chemistry 1993, 4 (2), 105-111.

43. Burggraaf, J.; Kamerling, I. M. C.; Gordon, P. B.; Schrier, L.; de Kam, M. L.; Kales, A. J.; Bendiksen, R.; Indrevoll, B.; Bjerke, R. M.; Moestue, S. A.; Yazdanfar, S.; Langers, A. M. J.; Swaerd-Nordmo, M.; Torheim, G.; Warren, M. V.; Morreau, H.; Voorneveld, P. W.; Buckle, T.; van Leeuwen, F. W. B.; Ødegårdstuen, L.-I.; Dalsgaard, G. T.; Healey, A.; Hardwick, J. C. $\mathrm{H}$. Detection of colorectal polyps in humans using an intravenously administered fluorescent peptide targeted against c-Met. Nature Medicine 2015, 21, 955.

44. Bunschoten, A.; van Willigen, D. M.; Buckle, T.; van den Berg, N. S.; Welling, M. M.; Spa, S. J.; Wester, H.-J.; van Leeuwen, F. W. B. Tailoring Fluorescent Dyes To Optimize a Hybrid RGD-Tracer. Bioconjugate Chemistry 2016, 27 (5), 1253-1258.

45. Gravesen, P.; Branebjerg, J.; Jensen, O. S. Microfluidics-a review. Journal of Micromechanics and Microengineering 1993, 3 (4), 168-182.

46. Whitesides, G. M. The origins and the future of microfluidics. Nature 2006, 442 (7101), 368-373.

47. Theberge, A. B.; Courtois, F.; Schaerli, Y.; Fischlechner, M.; Abell, C.; Hollfelder, F.; Huck, W. T. S. Microdroplets in Microfluidics: An Evolving Platform for Discoveries in Chemistry and Biology. Angewandte Chemie International Edition 2010, 49 (34), 5846-5868.

48. Rafeie, M.; Hosseinzadeh, S.; Taylor, R. A.; Warkiani, M. E. New insights into the physics of inertial microfluidics in curved microchannels. I. Relaxing the fixed inflection point assumption. Biomicrofluidics 2019, 13 (3), 034117.

49. Rafeie, M.; Hosseinzadeh, S.; Huang, J.; Mihandoust, A.; Warkiani, M. E.; Taylor, R. A. New insights into the physics of inertial microfluidics in curved microchannels. II. Adding an additive rule to understand complex cross-sections. Biomicrofluidics 2019, 13 (3), 034118.

50. Zhou, C.; Zhu, P.; Tian, Y.; Xu, M.; Wang, L. Engineering Micromotors with Droplet Microfluidics. ACS Nano 2019, 13 (6), 6319-6329.

51. Huang, X.; Tang, J.; Hu, L.; Bian, R.; Liu, M.; Cao, W.; Zhang, H. Arrayed microfluidic chip for detection of circulating tumor cells and evaluation of drug potency. Analytical Biochemistry 2019, 564-565, 64-71. 
52. Damhorst, G. L.; Murtagh, M.; Rodriguez, W. R.; Bashir, R. Microfluidics and Nanotechnology for Detection of Global Infectious Diseases. Proceedings of the Institute of Electrical and Electronics Engineers 2015, 103 (2), 150-160.

53. Dittrich, P. S.; Manz, A. Lab-on-a-chip: microfluidics in drug discovery. Nature Reviews Drug Discovery 2006, 5 (3), 210-218.

54. Duffy, D. C.; McDonald, J. C.; Schueller, O. J. A.; Whitesides, G. M. Rapid Prototyping of Microfluidic Systems in Poly(dimethylsiloxane). Analytical Chemistry 1998, 70 (23), 49744984.

55. McDonald, J. C.; Whitesides, G. M. Poly (dimethylsiloxane) as a material for fabricating microfluidic devices. Accounts of Chemical Research 2002, 35 (7), 491-499.

56. Di Iorio, D.; Verheijden, M. L.; van der Vries, E.; Jonkheijm, P.; Huskens, J. Weak Multivalent Binding of Influenza Hemagglutinin Nanoparticles at a Sialoglycan-Functionalized Supported Lipid Bilayer. ACS Nano 2019, 13 (3), 3413-3423.

57. Appel, J.; Akerboom, S.; Fokkink, R. G.; Sprakel, J. Facile One-Step Synthesis of Monodisperse Micron-Sized Latex Particles with Highly Carboxylated Surfaces. Macromolecules Rapid Communications 2013, 34 (16), 1284-1288.

58. Grace, D.; Fetsch, A. Chapter 1 - Staphylococcus aureus-A Foodborne Pathogen: Epidemiology, Detection, Characterization, Prevention, and Control: An Overview. In Staphylococcus aureus, Fetsch, A., Ed. Academic Press: 2018; pp 3-10.

59. Stefani, S.; Chung, D. R.; Lindsay, J. A.; Friedrich, A. W.; Kearns, A. M.; Westh, H.; MacKenzie, F. M. Meticillin-resistant Staphylococcus aureus (MRSA): global epidemiology and harmonisation of typing methods. International Journal of Antimicrobial Agents 2012, 39 (4), 273-282.

60. Gould, I. M.; David, M. Z.; Esposito, S.; Garau, J.; Lina, G.; Mazzei, T.; Peters, G. New insights into meticillin-resistant Staphylococcus aureus (MRSA) pathogenesis, treatment and resistance. International Journal of Antimicrobial Agents 2012, 39 (2), 96-104.

61. Kaper, J. B.; Nataro, J. P.; Mobley, H. L. T. Pathogenic Escherichia coli. Nature Reviews Microbiology 2004, 2 (2), 123-140.

62. Osman, K. M.; Kappell, A. D.; Elhadidy, M.; ElMougy, F.; El-Ghany, W. A. A.; Orabi, A.; Mubarak, A. S.; Dawoud, T. M.; Hemeg, H. A.; Moussa, I. M. I.; Hessain, A. M.; Yousef, H. M. Y. Poultry hatcheries as potential reservoirs for antimicrobial-resistant Escherichia coli: A risk to public health and food safety. Scientific Reports 2018, 8 (1), 5859. 


\section{Chapter 2}

\section{Covalently Bound Monolayers Obtained by Plasma Etching on Glass Surfaces}

Micropatterns of $\beta$-cyclodextrin ( $\beta$-CD) monolayers on glass are obtained by using a plasma etching approach with polydimethylsiloxane (PDMS) stamps. This simple and versatile approach provides a promising alternative to current techniques for creating patterns of covalently bound molecules. It was also possible to fabricate sub-10 $\mu \mathrm{m}$ sized features.

This chapter has been published as: Stan B.J. Willems, Laura M.I. Schijven, Anton Bunschoten, Fijs. W.B. van Leeuwen, Aldrik H. Velders, and Vittorio Saggiomo, Chemical Communications, 2019, 55, 7767-7670. doi: 10.1039/C9CC03791H 


\subsection{Introduction}

For decades, molecular monolayers on surfaces have been used for the fabrication of functional materials. Surfaces have been modified to tune both physical properties, e.g. wettability, and chemical properties, e.g. supramolecular interactions. ${ }^{1}$ The resulting monolayer assemblies on glass have been extensively studied with a plethora of molecular sensors. One way to study monolayer assembly is through using bright field or fluorescence microscopy. ${ }^{2-4}$ Within microscopy, spatial control of surface modification became important to allow validation within a single field of view. Therefore patterning of molecules on surfaces was introduced by Whitesides et al. in the form of microcontact printing $(\mu \mathrm{CP}) .^{5} \mu \mathrm{CP}$ is a soft lithographic technique wherein polydimethylsiloxane (PDMS) stamps are used for patterning surfaces, similar to Gutenberg's printing press. The patterned PDMS stamps are generally made from a silicon master wafer which contains features fabricated through UV-lithography. Importantly, this form of patterning allows positive and negative experimental controls to be placed in one field of view, which is a feature that promotes qualification of analytes. However, a major disadvantage within $\mu \mathrm{CP}$ is that the inking and patterning step requires a lengthy process of trial and error, due to issues such as solvent compatibility of PDMS stamps and lateral spreading of inks during printing. ${ }^{6,7}$ Moreover, a clean room is often required for reaching sub-10 $\mu \mathrm{m}$ resolution feature size within UV-lithography and therefore also $\mu \mathrm{CP}$. To circumvent the limitations of $\mu \mathrm{CP}$, alternative surface patterning techniques were developed such as dip-pen nanolithography, ${ }^{8,9} \mathrm{UV}$-initiated photografting, ${ }^{10,11}$ and laser ablation through light induced molecular adsorption technology. ${ }^{12,13}$ The wide adoption of these methodologies, however, may be limited by production costs.

As an alternative, plasma initiated patterning/plasma microcontact patterning $(\mathrm{P} \mu \mathrm{CP})$ was developed by Langowski et al. ${ }^{14}$ and Picone et al. ${ }^{15}$ for creating patterns of physisorbed proteins on surfaces. The high energetic plasma decomposes organic molecules on the surface, ${ }^{16-19}$ and a patterned PDMS stamp acts as a mask to control the exposure of the surface to the plasma. In contrast to plasma glass etching, which relies on $\mathrm{SF}_{6}, \mathrm{C}_{4} \mathrm{~F}_{8}$, or $\mathrm{CHF}_{3}$ as a fluorine based etchant for removing Si atoms, ${ }^{20}$ in this case, air is used as etching gas and the plasma decomposes only the organic molecules present on the surface, in a process known as isotropic 'chemical etching'. ${ }^{21}$ So far, the $\mathrm{P} \mu \mathrm{CP}$ technique has been predominantly used for creating patterns of physisorbed molecules for biological applications. We hypothesized that this concept is more versatile and can also be used to obtain patterns on covalently coated 


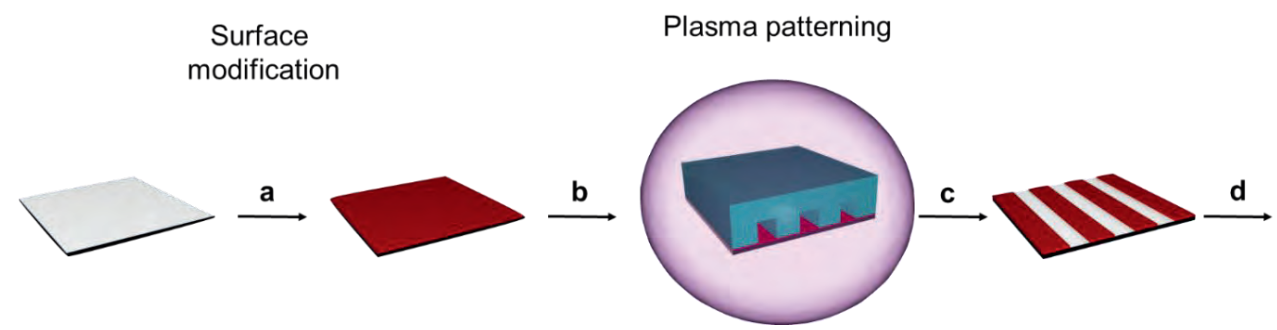

Fig. 2.1: Scheme showing principle of $\mathrm{P} \mu \mathrm{CP}$ on surfaces: a) (Chemical) covalent glass slide modification with desired functionality, b) placing of PDMS mask on modified slide for plasma patterning, c) localized removal of surface functionality using plasma etching to yield patterns on glass surface, and d) further surface functionalization steps.

surfaces. To demonstrate the potential of this approach, we focused on covalent surface modification starting from the organosilane monolayer assembly. The $\mathrm{P} \mu \mathrm{CP}$ step can already be applied after organosilane modification or at a later stage to create patterns of molecules by protecting part of the surface during plasma treatment with a patterned PDMS stamp (Fig. 2.1). Further modification steps can also be carried out on the patterned surface and characterization is possible through labeling with specific targeting fluorophores for analysis of the surface by (fluorescence) microscopy. The ability to use $\mathrm{P} \mu \mathrm{CP}$ for patterning covalently attached monolayers could open the field of surface functionalization without the lengthy process of $\mu \mathrm{CP}$ optimization or using complicated and/or expensive techniques.

We investigated the use of $\beta$-cyclodextrin $(\beta-C D)$ on glass surfaces as a model for the covalent attachment of monolayers and creating patterns using $\mathrm{P} \mu \mathrm{CP}$ methodology. The multiple steps for obtaining a monolayer of $\beta-\mathrm{CD}$ are well studied in literature, known as the 'molecular printboard',22,23 in which covalently, surface bound $\beta-\mathrm{CD}$ has been used for immobilizing non-covalently guest molecules ${ }^{2,4,24,25}$ and nanoparticles. ${ }^{3,26}$ Within the field of supramolecular host-guest chemistry, adamantane is often used as a guest molecule because of its high affinity for $\beta-\mathrm{CD}$ due to its hydrophobic nature, size, and possibility for multivalent interactions. Therefore, the multiple steps for obtaining a monolayer of $\beta-\mathrm{CD}$ allows studying the effect of $\mathrm{P} \mu \mathrm{CP}$ on different molecular moieties, with respect to retaining the host-guest functionality of $\beta-C D$ for the immobilization of adamantane functionalized molecules. 


\subsection{Experimental Section}

\section{General}

Chemicals were purchased from Sigma Aldrich/Merck unless mentioned otherwise. Knittel (Germany) borosilicate glass cover slips (18x18 mm, No. 1) were used as glass substrates for functionalization experiments. Hyper pure polished silicon wafers $(76.2 \mathrm{~mm}$ diameter, University Wafer, USA) were used as silicon masters. A photomask template containing patterns, with different feature and spacing sizes, was designed and ordered from JD photo data (UK). Deionized (DI) water was used in all the experiments. Cyanine 5diadamantane (Cy5-Ad2) was synthesized by Dr. Mark Rood from the Interventional Molecular Imaging lab at the Leiden University Medical Centre (LUMC, Leiden, the Netherlands). ${ }^{27}$

\section{Instrumentation}

For the preparation of the silicon master, a standard UV-lithography set-up was used. The negative photoresist on the silicon wafer was irradiated with a UV lamp at $\lambda=350 \mathrm{~nm}$ at $50 \%$ intensity. An Inseto Plasma Etch, Inc. PE-25 benchtop air plasma cleaner was used for plasma cycles of $1 \mathrm{~min}$ at its maximum RF plasma power of $100 \mathrm{~W}$ with an air flow of $\sim 5-10$ cc/min, which allowed for a vacuum pressure of 200-250 mTorr within the chamber during plasma treatment. ${ }^{\dagger}$ An RC6 Chemistry Hybrid Pump from Vauubrand (vacuum of $2 \times 10^{-3}$ mbar) was used for applying high vacuum condition in desiccators. The functionalized glass surface were imaged in air, using a Leica DMi8 epifluorescence microscope with 40X (oil immersion), 10X, $5 \mathrm{X}$ or $2.5 \mathrm{X}$ magnification objective lens, beam intensity of $100 \%$ and an exposure time of $278 \mathrm{~ms}$. The Cy5 fluorescence was excited at a wavelength $\left(\lambda_{\mathrm{ex}}\right)$ of 590-650 $\mathrm{nm}$ and emission $\left(\lambda_{\mathrm{em}}\right)$ was collected at $662-738 \mathrm{~nm}$ (using the Y5 filter cube), rhodamine B isothiocyanate (RITC) at $\lambda_{\mathrm{ex}}$ of $541-551 \mathrm{~nm}$ and $\lambda_{\mathrm{em}}$ of $662-738 \mathrm{~nm}$ (RHOD filter cube). The results were analyzed with FIJI software (ImageJ). ${ }^{28}$ The images were rotated in such a way that the patterns were positioned vertically. Then, a square was drawn over the patterns and the averaged gray values were collected within that square. The profile plots were also corrected for fluorescent beam curvature by acquiring profile plots of a fully fluorescent $\mathrm{Cy} 5$ slide with the same objective (see Fig. S3), normalizing this curvature plot, and then multiplying the

\footnotetext{
${ }^{\dagger}$ Air plasma works similar to oxygen plasma and the surface cleaning is based on chemical, isotropic etching with highly reactive oxygen radicals. ${ }^{21}$
} 
fluorescent intensity of line profile plots by the inverse of the curvature plot. The corrected gray values (a.u.) were then normalized for the maximum value and plotted against the distance $(\mu \mathrm{m})$.

\section{Glass surface functionalization}

The general glass surface functionalization with $\beta$-cyclodextrin was performed as described by Onclin et al. ${ }^{23}$ Glass microscope cover slips were cleaned and oxidized with piranha solution $\left(\mathrm{H}_{2} \mathrm{SO}_{4}(95-98 \%) / \mathrm{H}_{2} \mathrm{O}_{2}(35 \%)\right.$, 3.33:1 v/v; Warning! Piranha solutions must be handled with caution as they may unexpectedly detonate) for 45 minutes, rinsed with large amounts DI water, and dried under $\mathrm{N}_{2}$. The glass slides were placed in a high vacuum, preheated desiccator together with a glass vial containing $1 \mathrm{~mL}$ of 3 -aminopropyltriethoxysilane (APTES, 99\%) and placed in an oven at $70{ }^{\circ} \mathrm{C}$ overnight for chemical vapor deposition (CVD) of APTES. Following amine monolayer formation, the glass slides were removed from the desiccator and rinsed with toluene (HPLC grade, VWR, the Netherlands) and dichloromethane (DCM, VWR). The glass slides were then cured for at least 1 hour in the oven at $70{ }^{\circ} \mathrm{C}$. Next, the glass slides were immersed in $0.1 \mathrm{M}$ 1,4-phenylene diisothiocyanate (PDITC, TCI Chemicals Belgium) in anhydrous toluene (max $0.002 \% \mathrm{H}_{2} \mathrm{O}$, VWR) for 2 hours under argon atmosphere to yield isothiocyanate bearing layers. Following the immersion, the surfaces were rinsed with toluene and DCM and incubated in $0.72 \mathrm{mM}$ heptakis amino $\beta$-cyclodextrin $(\beta-\mathrm{CD}$, Cyclodextrin Shop, the Netherlands) in aqueous solution at $\mathrm{pH} 8.0$ (reached by adding small amount of $1 \mathrm{M} \mathrm{NaOH}$ ). This incubation was carried out for at least 2 hours. Surfaces were then rinsed with DI water and dried with nitrogen.

\section{Plasma microcontact patterning (P $\mu \mathrm{CP})$}

Stamps were fabricated by using a replica molding technique from Whitesides. ${ }^{5} \mathrm{~A}$ silicon master was first fabricated by spin-coating at $1500 \mathrm{rpm}$ with SU-8 2025 photoresist (to yield $50 \mu \mathrm{m}$ thick photoresist layer) from Microchem and then treated using UV photolithography with a mask containing $150 \mu \mathrm{m}$ patterns and $50 \mu \mathrm{m}$ pattern spacing. The silicon master and a separate glass vial containing $100 \mu \mathrm{L} \mathrm{mL}$ of trichloro $(1 \mathrm{H}, 1 \mathrm{H}, 2 \mathrm{H}, 2 \mathrm{H}-$ perfluorooctyl)silane (PFOTS, 97\%) were introduced into a desiccator under vacuum for overnight CVD. After incubation with PFOTS, the wafer was cleaned with isopropanol and dried with nitrogen. Stamps were prepared by casting a 10:1 (w/w) mixture of 
poly(dimethylsiloxane) (PDMS) and curing agent (Sylgard 184, Dow Corning) onto the silicon master with $150 \mu \mathrm{m}$ patterns and $50 \mu \mathrm{m}$ pattern spacing. After overnight curing at $70{ }^{\circ} \mathrm{C}$, the PDMS stamps were cut out the master to ca $0.75 \mathrm{~cm}^{2}$ and sonicated in ethanol to remove low molecular weight PDMS. The PDMS stamps were then brought into conformal contact with the freshly prepared functionalized glass surfaces. The glass substrates were then placed in the plasma cleaner at high energy for 4 cycles of 1 minute each. Surfaces were rinsed again with DI water and dried with nitrogen.

\section{Fluorescence microscopy}

APTES patterned glass surfaces were incubated with $1 \mathrm{mM}$ RITC dissolved in methanol for 5 minutes. The surface was then rinsed thoroughly with methanol to remove excess RITC and then dried with nitrogen. RITC stained APTES patterns were then visualized with the microscope. For $\beta-\mathrm{CD}$ functionalized surfaces, $0.28 \mu \mathrm{M}$ of $\mathrm{Cy} 5-\mathrm{Ad}_{2}$ in phosphate buffered saline (PBS: $10 \mathrm{mM} \mathrm{Na} \mathrm{HPO}_{4}, 1.8 \mathrm{mM} \mathrm{KH} 2 \mathrm{PO}_{4}, 137 \mathrm{mM} \mathrm{NaCl}, 2.7 \mathrm{mM} \mathrm{KCl}, \mathrm{pH} 7.4$ ) was incubated on the glass surface for 15 minutes. The surface was then rinsed thoroughly with DI water and dried under a stream of nitrogen before imaging with the microscope. 


\subsection{Results and Discussion}

The $\beta$-CD glass surface functionalization works as displayed in Fig. 2.2a: 1) silanization of glass surface silanol groups using 3-aminopropyltriethoxysilane (APTES) for obtaining an amine layer, 2) covalent attachment of heptakis amino $\beta$-CD addition via 1,4-phenylene diisothiocyanate (PDITC) cross-linking, and 3) visualization of $\beta$-CD patterns with fluorescent divalent guest molecule Cyanine 5-diadamantane $\left(\mathrm{Cy} 5-\mathrm{Ad}_{2}\right) .{ }^{27} \mathrm{P} \mu \mathrm{CP}$ was carried out after the functionalization of the glass slide with APTES (Exp. 1), after the attachment of $\beta$-CD (Exp. 2), and also after the non-covalent immobilization of Cy5-Ad2 (Exp. 3) ${ }^{\ddagger}$ For Exp. 1-2, Cy5$\mathrm{Ad}_{2}$ dye was subsequently added to functionalized surfaces to label the $\beta$-CD patterns. Each single experiment was then validated using fluorescence microscopy. In addition, we also created more complex patterns using $\mathrm{P} \mu \mathrm{CP}$ on fully functionalized $\beta-\mathrm{CD}$ surfaces which also generated sub-10 $\mu \mathrm{m}$ sized features. Importantly, all these experiments, including the fabrication of patterned silicon wafers for the PDMS stamps, were carried out without the use of a clean room.

For Exp. 1, after APTES monolayer assembly and creation of patterns through $\mathrm{P} \mu \mathrm{CP}$, the surface was first validated by addition of Rhodamine B isothiocyanate (RITC), as an additional control (Fig. S1). RITC is highly reactive towards amines and can therefore be used to fluorescently label APTES patterns. Patterns $(150 \mu \mathrm{m})$ of bright fluorescent lines were observed, while dark regions $(50 \mu \mathrm{m})$ show no fluorescence as they were exposed to plasma treatment before addition of RITC. This indicated that plasma etching effectively removed all covalently bound APTES from the glass surface outside of the PDMS covered areas and that the protected APTES molecules were still functional. The patterned APTES surfaces were further reacted with PDITC and consecutively with heptakis amino $\beta$-CD (Fig. 2.2a). Addition of the fluorophore $\mathrm{Cy} 5-\mathrm{Ad}_{2}$ was carried out to visualize surface bound $\beta-\mathrm{CD}$ through host-guest interactions. Dark regions were again observed between $\beta$-CD lines, which indicates that $\beta$-CD was successfully cross-linked to APTES patterns. Also in this case, clear fluorescent lines (150 $\mu \mathrm{m})$ of $\mathrm{Cy} 5-\mathrm{Ad}_{2}$ immobilized to $\beta-\mathrm{CD}$ patterns were observed (Exp. 1, Fig. 2.2b, larger overview in Fig. S5-S6). In Exp. 2, fully covered surfaces of $\beta-C D$ were P $\mu$ CP treated and

\footnotetext{
* Patterning isothiocyanate moieties through $\mathrm{P} \mu \mathrm{CP}$ was unsuccessful. We hypothesize that these groups are too reactive to remain intact for a prolonged duration and should be reacted soon after this modification step.
} 


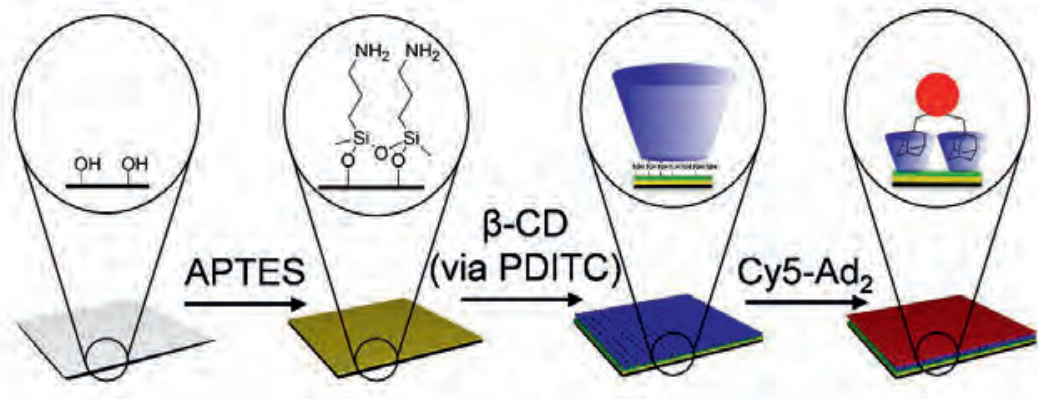

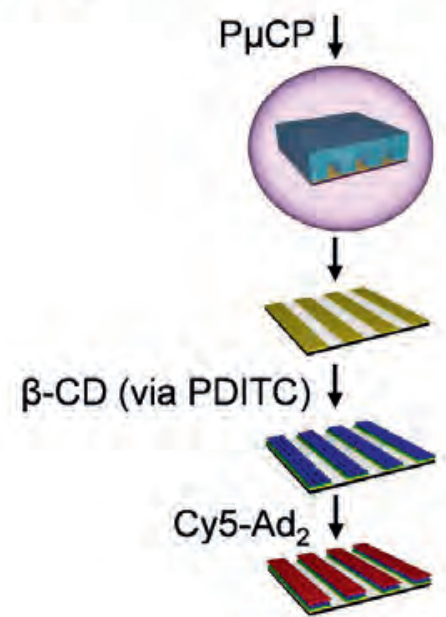

Exp. 1

b
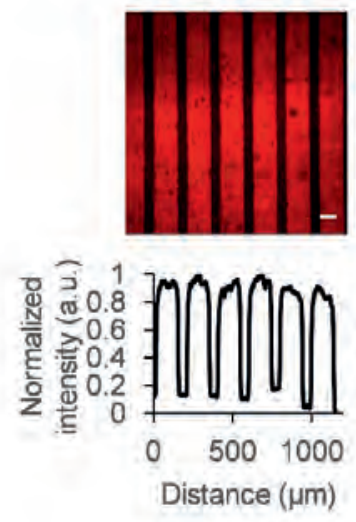

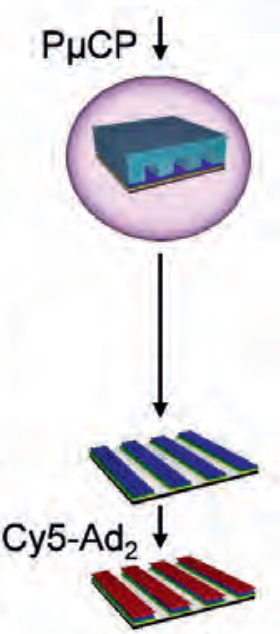

Exp. 2
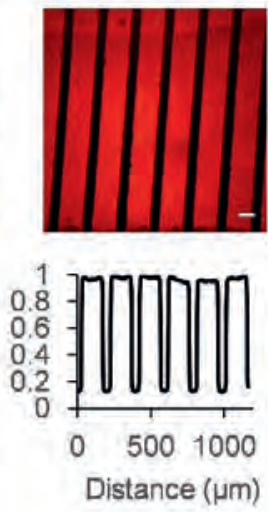

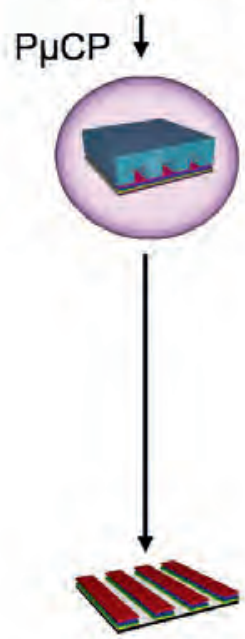

Exp. 3
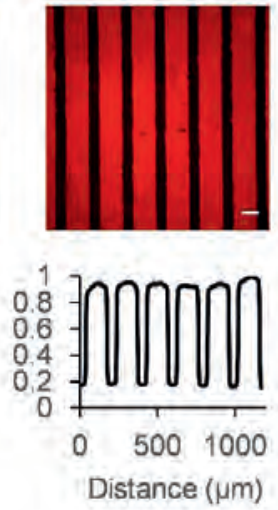

Fig. 2.2: a) Scheme showing the $\beta-C D$ glass surface functionalization steps and immobilization of fluorescent guest molecule $\mathrm{Cy} 5-\mathrm{Ad}_{2}$. P $\mu \mathrm{CP}$ was applied either after APTES monolayer assembly (Exp. 1), $\beta$-CD attachment (Exp. 2), or after Cy5-Ad 2 immobilization (Exp. 3). b) Fluorescence microscope images of Exp. 1: Cy5-Ad 2 addition to $\beta$-CD after PDITC mediated attachment of $\beta$-CD to P $\mu$ CP APTES patterns, Exp. 2: Cy5-Ad addition $^{-}$ to $\mathrm{P} \mu \mathrm{CP} \beta-\mathrm{CD}$ after PDITC mediated attachment of $\beta-\mathrm{CD}$ to fully covered APTES surface, and Exp. 3: $\mathrm{P} \mu \mathrm{CP}$ of $\mathrm{Cy} 5-\mathrm{Ad}_{2}$ after its immobilization to fully covered $\beta-\mathrm{CD}$ surface. Profile plots showing the gray value intensity vs the distance in $\mu \mathrm{m}$ are depicted below the fluorescence images and are corrected for beam curvature (see Fig. S2-S4 for explanation). Microscope images were captured with 10x objective and scale bars are $100 \mu \mathrm{m}$. 
subsequently incubated with Cy5-Ad2 (Fig. 2.2a). The selective immobilization of Cy5- $\mathrm{Ad}_{2}$ is clearly observed on $\beta$-CD patterns as bright fluorescent lines (Exp. 2, Fig. 2.2b, larger overview in Fig. S5-S6). In Exp. 3, we used $\mathrm{P} \mu \mathrm{CP}$ in the final step of the surface modification process after addition of $\mathrm{Cy} 5-\mathrm{Ad}_{2}$ (Fig. 2.2a). Clear patterns of host-guest immobilized Cy5-Ad2 are also observed in Fig. 2.2b for Exp. 3. The generated patterns for Experiments 1-3 all show clear contrast between the desired feature and the spacing, which showcases the reproducibility of the technique. Moreover, the features of fabricated PDMS stamps were compared with the resulting $\mathrm{P} \mu \mathrm{CP}$ patterns through repeating Exp. 3 from Fig. 2.2a and leaving the PDMS stamp on the surface during microscope imaging (Fig. S7). These images show that the fluorescent patterns are in accordance with the PDMS stamp features when comparing the Cy5 channel with the brightfield channel, which is also further confirmed with an overlay image (Fig. S8). Therefore, using $\mathrm{P} \mu \mathrm{CP}$ methodology for creating patterns of covalently attached monolayers is successful at multiple steps within a functionalization protocol allowing great versatility.

We also explored a simple approach, using $\mathrm{P} \mu \mathrm{CP}$, for creating intricate and smaller $\beta$ CD patterns than the $150 \mu \mathrm{m}$ line features of the applied PDMS stamp. In general, the fabrication of sub-10 $\mu \mathrm{m}$ patterns on surfaces through conventional techniques, such as UVlithography, usually requires a clean room. Here, 'cross'-P $\mu \mathrm{CP}$ can be used to create unconventional small features, starting from conventional large patterns. After obtaining a fully functionalized $\beta-C D$ surface according to the protocol shown in Fig. 2.2a, the P $\mu C P$ step for creating patterns was slightly altered by rotating the PDMS mask between multiple plasma cycles (Fig. 2.3a). The first plasma treatment step was carried out, as previously, by placing the PDMS stamp on the functionalized surface at $0^{\circ}$ (vertical lines). This step was then followed by several rotations of the PDMS stamp between plasma cycles: $\pm 90^{\circ}, \pm 45^{\circ}$, and finally $\pm 90^{\circ}$, all with respect to the previous step. This was done in order to remove more $\beta$-CD molecules from the surface through plasma etching. $\mathrm{Cy} 5-\mathrm{Ad}_{2}$ addition to the treated surface then yielded a variety of shapes when imaged on the microscope (Fig. 2.3b). As observed in Fig. 2.3b(iv), we also managed to fabricate distinguishable sub-10 $\mu \mathrm{m}$ features of $\beta-\mathrm{CD}$ on the same surface by using cross-P $\mu \mathrm{CP}$. The result shows clear contrast between functionalized and nonfunctionalized areas and are agreeable with the expected patterns depicted in the scheme in Fig. 2.3a. 


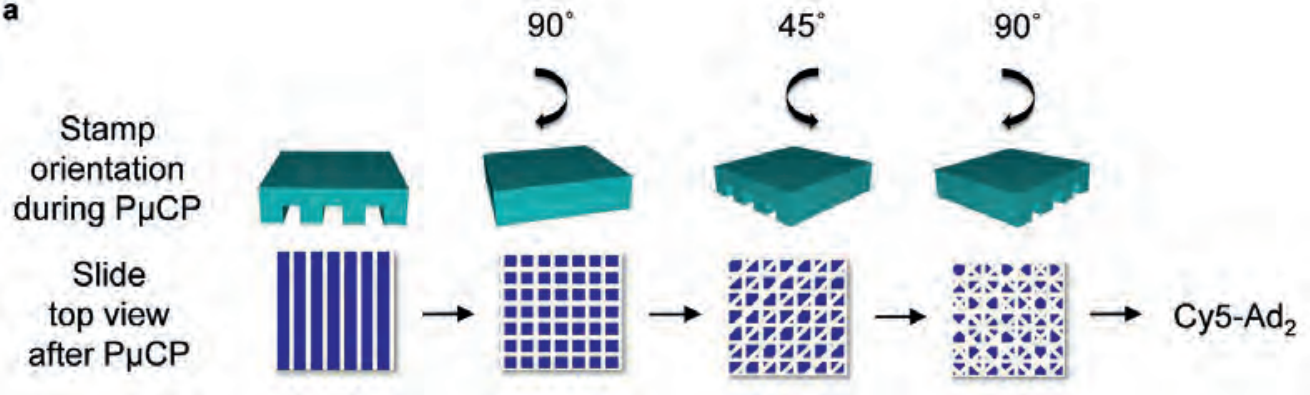

b
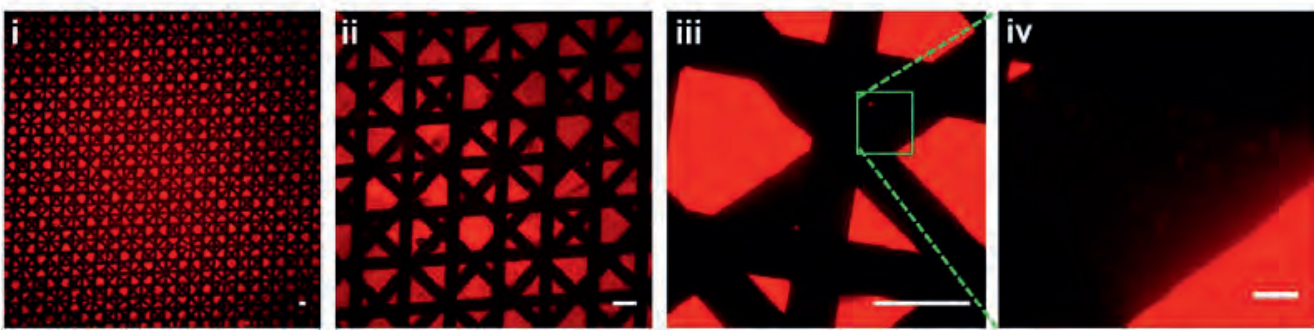

Fig. 2.3: a) Scheme showing the concept of changing the stamp orientation in between $\mathrm{P} \mu \mathrm{CP}$ cycles on modified glass surface. The expected outcome of patterns on top of the glass surface is shown below the PDMS stamps. Cy5-Ad $\mathrm{As}_{2}$ added only after all 'cross'-P $\mu$ CP steps. b) Fluorescence microscope images of $\beta$-CD surface after different $\mathrm{P} \mu \mathrm{CP}$ treatments and addition of Cy5- $\mathrm{Ad}_{2}$. Images were captured on the same glass slide with i) $2.5 \mathrm{x}$ objective, ii) $10 \mathrm{x}$ objective and iii) 40x objective. A digital zoom of iii) is depicted in iv) to showcase a triangular shape with a size around $5 \mu \mathrm{m}$. All scale bars except for iv) are $100 \mu \mathrm{m}$. The scale bar in iv) is $10 \mu \mathrm{m}$. 


\subsection{Conclusion}

In this work, we described how a micropatterning technique $(\mathrm{P} \mu \mathrm{CP})$ based on the spatially controlled removal of molecules through plasma treatment and PDMS stamps, can easily be applied to monolayers of covalently bound molecules on surfaces. We show that $\mathrm{P} \mu \mathrm{CP}$ can be used to create patterns during different steps and thus on different molecular moieties within the extensive $\beta$-CD surface functionalization, while also retaining the reactivity of the amine monolayer and the supramolecular functionality of the $\beta$-CD layer. We believe that, owing to its simplicity, $\mathrm{P} \mu \mathrm{CP}$ is more straightforward and reproducible than current micropatterning techniques for covalently bound molecules. Moreover, by using 'cross'-P $\mu \mathrm{CP}$, a methodology which works contrapositive to additive manufacturing techniques, we fabricated sub-10 $\mu \mathrm{m}$ features. This simple technique can also open the concept of micropatterning to chemists who do not have access or experience in clean-room technology or microfabrication. 


\subsection{References}

1. Ahn, Y.; Jang, Y.; Selvapalam, N.; Yun, G.; Kim, K. Supramolecular Velcro for Reversible Underwater Adhesion. Angewandte Chemie International Edition 2013, 52 (11), 3140-3144.

2. Mulder, A.; Onclin, S.; Peter, M.; Hoogenboom, J. P.; Beijleveld, H.; ter Maat, J.; Garcia-Parajo, M. F.; Ravoo, B. J.; Huskens, J.; van Hulst, N. F.; Reinhoudt, D. N. Molecular printboards on silicon oxide: lithographic patterning of cyclodextrin monolayers with multivalent, fluorescent guest molecules. Small 2005, 1 (2), 242-253.

3. Dorokhin, D.; Hsu, S.-H.; Tomczak, N.; Reinhoudt, D. N.; Huskens, J.; Velders, A. H.; Vancso, G. J. Fabrication and Luminescence of Designer Surface Patterns with $\beta$-Cyclodextrin Functionalized Quantum Dots via Multivalent Supramolecular Coupling. ACS Nano 2010, 4 (1), 137-142.

4. Gonzalez-Campo, A.; Hsu, S. H.; Puig, L.; Huskens, J.; Reinhoudt, D. N.; Velders, A. $\mathrm{H}$. Orthogonal covalent and noncovalent functionalization of cyclodextrin-alkyne patterned surfaces. Journal of the American Chemical Society 2010, 132 (33), 11434-11436.

5. Xia, Y.; Whitesides, G. M. Soft Lithography. Angewandte Chemie International Edition 1998, 37 (5), 550-575.

6. Lee, J. N.; Park, C.; Whitesides, G. M. Solvent compatibility of poly (dimethylsiloxane)-based microfluidic devices. Analytical chemistry 2003, 75 (23), 6544-6554.

7. Perl, A.; Reinhoudt, D. N.; Huskens, J. Microcontact Printing: Limitations and Achievements. Advanced Materials 2009, 21 (22), 2257-2268.

8. Piner, R. D.; Zhu, J.; Xu, F.; Hong, S.; Mirkin, C. A. "Dip-Pen" Nanolithography. Science 1999, 283 (5402), 661-663.

9. Wu, C.-C.; Reinhoudt, D. N.; Otto, C.; Subramaniam, V.; Velders, A. H. Strategies for Patterning Biomolecules with Dip-Pen Nanolithography. Small 2011, 7 (8), 989-1002.

10. Zahner, D.; Abagat, J.; Svec, F.; Fréchet, J. M. J.; Levkin, P. A. A Facile Approach to Superhydrophilic-Superhydrophobic Patterns in Porous Polymer Films. Advanced Materials 2011, 23 (27), 3030-3034.

11. Geyer, F. L.; Ueda, E.; Liebel, U.; Grau, N.; Levkin, P. A. SuperhydrophobicSuperhydrophilic Micropatterning: Towards Genome-on-a-Chip Cell Microarrays. Angewandte Chemie International Edition 2011, 50 (36), 8424-8427.

12. Strale, P.-O.; Azioune, A.; Bugnicourt, G.; Lecomte, Y.; Chahid, M.; Studer, V. Multiprotein Printing by Light-Induced Molecular Adsorption. Advanced Materials 2015, 28 (10), 2024-2029.

13. Decock, J.; Schlenk, M.; Salmon, J.-B. In situ photo-patterning of pressure-resistant hydrogel membranes with controlled permeabilities in PEGDA microfluidic channels. Lab on a Chip 2018, 18 (7), 1075-1083.

14. Langowski, B. A.; Uhrich, K. E. Microscale plasma-initiated patterning ( $\mu$ pip). Langmuir 2005, 21 (23), 10509-10514.

15. Picone, R.; Baum, B.; McKendry, R. Chapter 5 - Plasma Microcontact Patterning $(\mathrm{P} \mu \mathrm{CP})$ : A Technique for the Precise Control of Surface Patterning at Small-Scale. In Methods in Cell Biology, Piel, M.; Théry, M., Eds. Academic Press: 2014; Vol. 119, pp 73-90.

16. Terpilowski, K.; Rymuszka, D. Surface properties of glass plates activated by air, oxygen, nitrogen and argon plasma. Glass Physics and Chemistry 2016, 42 (6), 535-541.

17. Homola, T.; Matoušek, J.; Kormunda, M.; Wu, L. Y. L.; Černák, M. Plasma Treatment of Glass Surfaces Using Diffuse Coplanar Surface Barrier Discharge in Ambient Air. Plasma Chemistry and Plasma Processing 2013, 33 (5), 881-894. 
18. DeRosa, R. L.; Schader, P. A.; Shelby, J. E. Hydrophilic nature of silicate glass surfaces as a function of exposure condition. Journal of Non-Crystalline Solids 2003, 331 (1-3), 32-40.

19. Alam, A.; Howlader, M.; Deen, M. The effects of oxygen plasma and humidity on surface roughness, water contact angle and hardness of silicon, silicon dioxide and glass. Journal of Micromechanics and Microengineering 2014, 24 (3), 035010.

20. Kolari, K.; Saarela, V.; Franssila, S. Deep plasma etching of glass for fluidic devices with different mask materials. Journal of Micromechanics and Microengineering 2008, 18 (6), 064010 .

21. Nageswaran, G.; Jothi, L.; Jagannathan, S. Chapter 4 - Plasma Assisted Polymer Modifications. In Non-Thermal Plasma Technology for Polymeric Materials, Thomas, S.; Mozetič, M.; Cvelbar, U.; Špatenka, P.; K.M, P., Eds. Elsevier: 2019; pp 95-127.

22. Ludden, M. J. W.; Reinhoudt, D. N.; Huskens, J. Molecular printboards: versatile platforms for the creation and positioning of supramolecular assemblies and materials. Chemical Society Reviews 2006, 35 (11), 1122-1134.

23. Onclin, S.; Mulder, A.; Huskens, J.; Ravoo, B. J.; Reinhoudt, D. N. Molecular printboards: monolayers of beta-cyclodextrins on silicon oxide surfaces. Langmuir 2004, 20 (13), 5460-5466.

24. Nijhuis, C. A.; Yu, F.; Knoll, W.; Huskens, J.; Reinhoudt, D. N. Multivalent Dendrimers at Molecular Printboards: Influence of Dendrimer Structure on Binding Strength and Stoichiometry and Their Electrochemically Induced Desorption. Langmuir 2005, 21 (17), 78667876.

25. Huskens, J.; Deij, M. A.; Reinhoudt, D. N. Attachment of Molecules at a Molecular Printboard by Multiple Host-Guest Interactions. Angewandte Chemie International Edition 2002, 41 (23), 4467-4471.

26. Mahalingam, V.; Onclin, S.; Peter, M.; Ravoo, B. J.; Huskens, J.; Reinhoudt, D. N. Directed self-assembly of functionalized silica nanoparticles on molecular printboards through multivalent supramolecular interactions. Langmuir 2004, 20 (26), 11756-11762.

27. Rood, M. T. M.; Spa, S. J.; Welling, M. M.; ten Hove, J. B.; van Willigen, D. M.; Buckle, T.; Velders, A. H.; van Leeuwen, F. W. B. Obtaining control of cell surface functionalizations via Pre-targeting and Supramolecular host guest interactions. Scientific Reports 2017, 7, 39908.

28. Schindelin, J.; Arganda-Carreras, I.; Frise, E.; Kaynig, V.; Longair, M.; Pietzsch, T.; Preibisch, S.; Rueden, C.; Saalfeld, S.; Schmid, B. Fiji: an open-source platform for biologicalimage analysis. Nature Methods 2012, 9 (7), 676-682. 


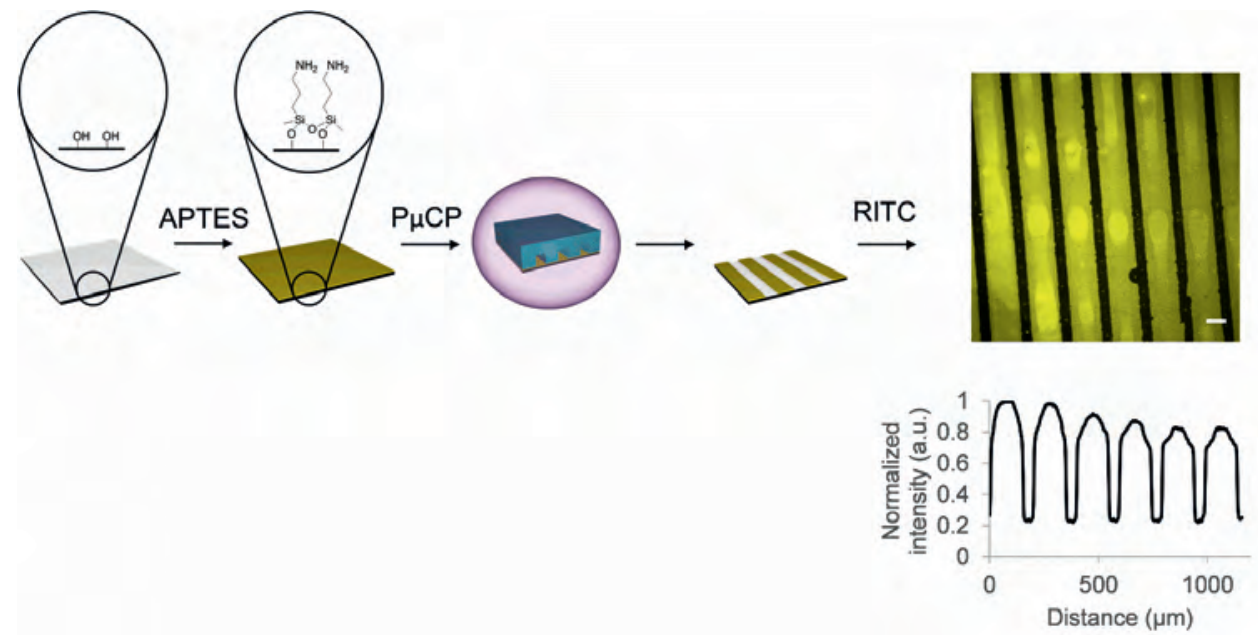

Fig. S1: Validation of APTES patterns after P $\mu$ CP (Exp. 1) using RITC. The scale bar in the microscope image is $100 \mu \mathrm{m}$. The profile plot below is already corrected for beam curvature.
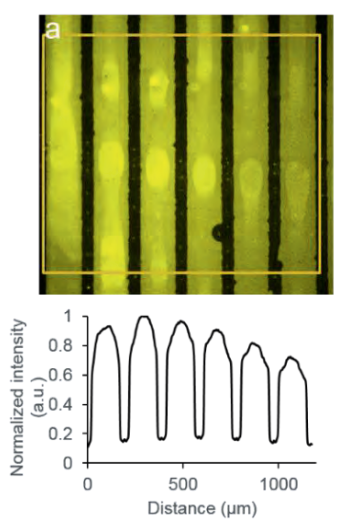
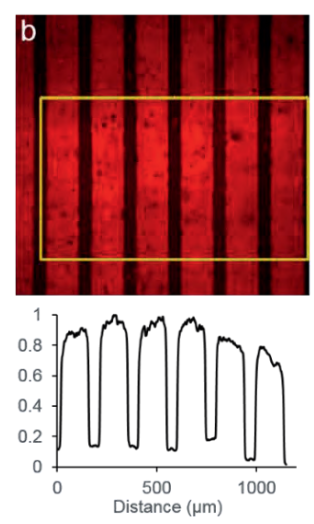
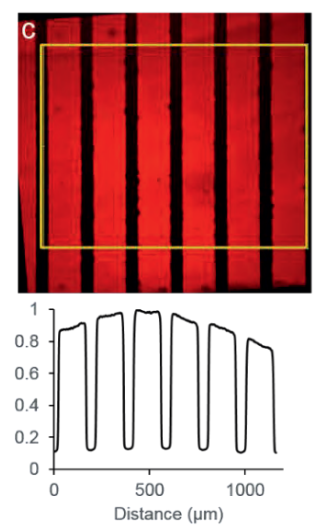
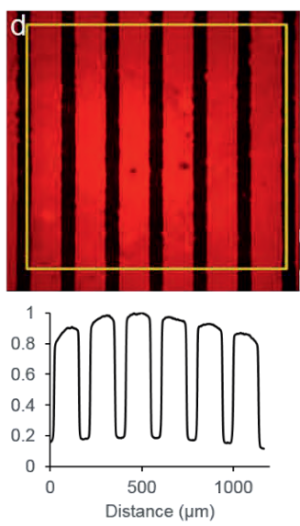

Fig. S2: Images before beam curvature correction. a) APTES P $\mu C P$ and RITC, b) Exp. 1: APTES P $\mu C P$-PDITC$\beta-\mathrm{CD}$ and $\mathrm{Cy} 5-\mathrm{Ad}_{2}$, c) Exp. 2: APTES-PDITC- $\beta-\mathrm{CD} \mathrm{P} \mu \mathrm{CP}$ and $\mathrm{Cy} 5-\mathrm{Ad}_{2}$ and d) Exp. 3: APTES-PDITC- $\beta$-CDCy5- $\mathrm{Ad}_{2} \mathrm{P} \mu \mathrm{CP}$. The yellow outline on each image marks the area used for creating the profile plots in Fiji. 

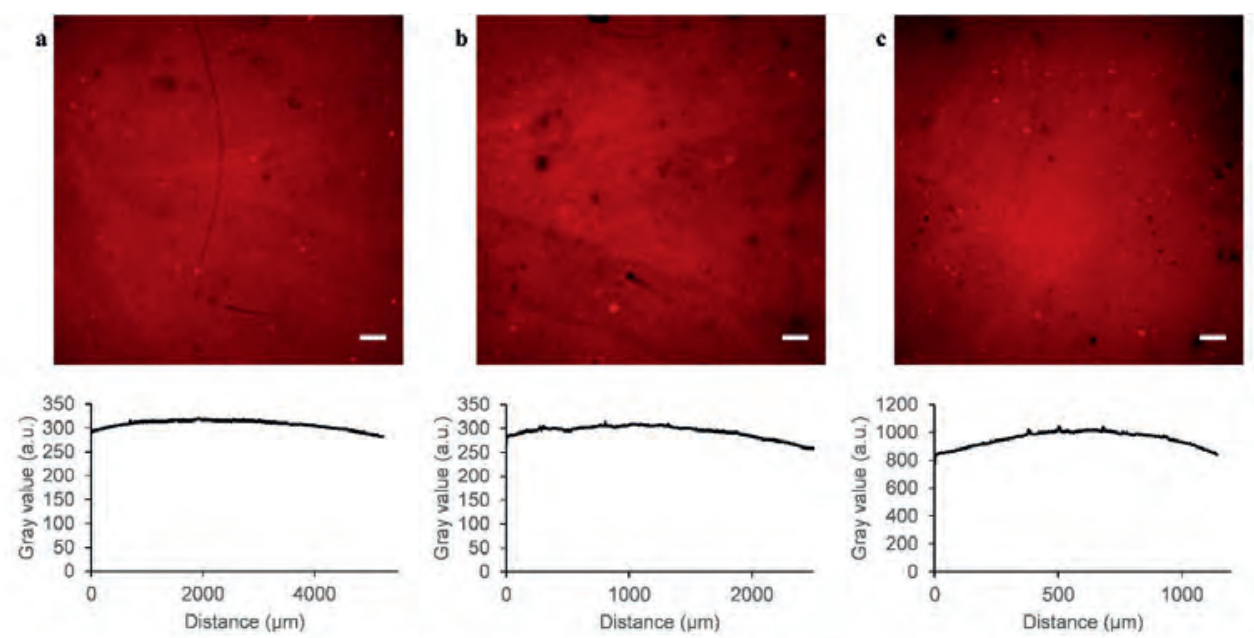

Fig. S3: Profile plots of beam curvature of Cy5-Ad 2 fluorescent glass slides using a) $2.5 x$ objective, b) $5 x$ objective, and c) 10x objective. These plots were used to correct for the beam curvature in line profile plots by first normalizing the intensity values, then inverting the values and multiplying them with profile plot intensity values from Fig. S4 for the constituent objective. Scale bars in images are a) $400 \mu \mathrm{m}$, b) $200 \mu \mathrm{m}$ and c) $100 \mu \mathrm{m}$

a
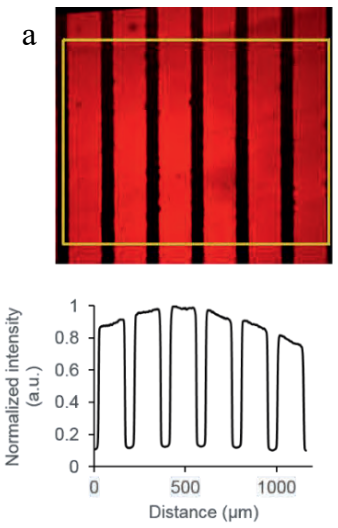

b
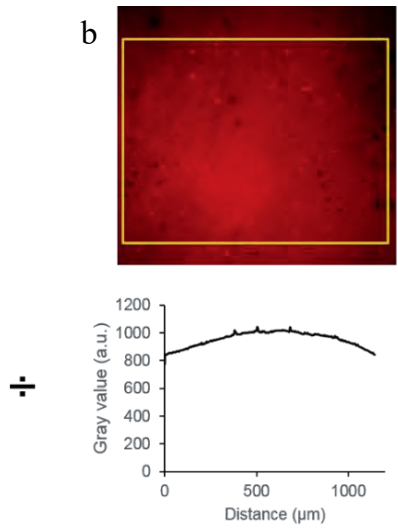

\section{$\mathrm{c}$}
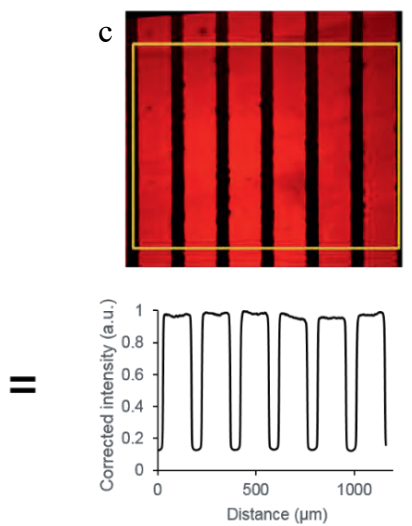

Fig. S4: Scheme depicting how the beam correction was carried out over an image using Fig. S3c and Fig. S4c as an example. a) non-corrected, normalized intensity shown in profile plot. b) intensity plot of a fully covered slide. c) corrected profile plot after dividing profile plot of a) with normalized profile plot of c). 

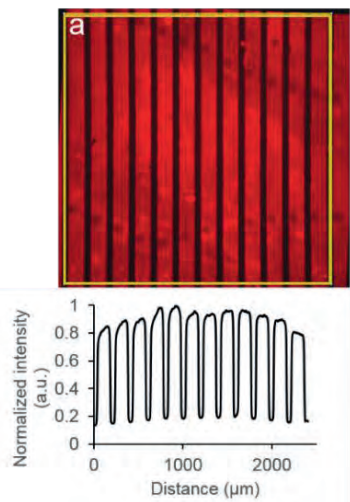
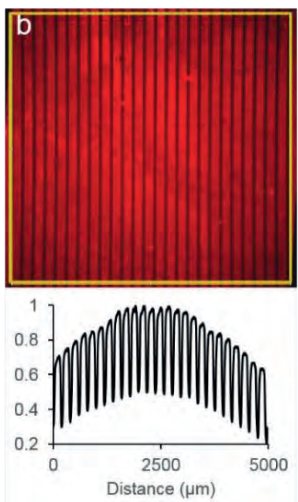
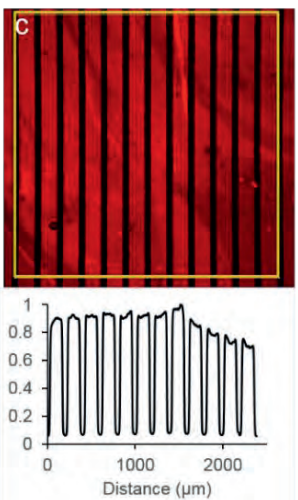
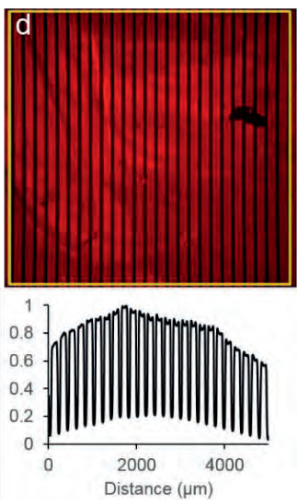

Fig. S5: Larger overview of experiments from Fig. 2 showing images of Experiment 1 with 2.5x (a) and 5x objective (b) and Experiment 2 with $2.5 \mathrm{x}$ (c) and $5 \mathrm{x}$ objective (d). Scale bars for $2.5 \mathrm{x}$ objective images are $400 \mu \mathrm{m}$ and for $5 \mathrm{x}$ objective images are $200 \mu \mathrm{m}$. Constituent profile plots for images (a)-(d) are before beam curvature correction. Yellow outline on each images mark the area used for creating the profile plot

a
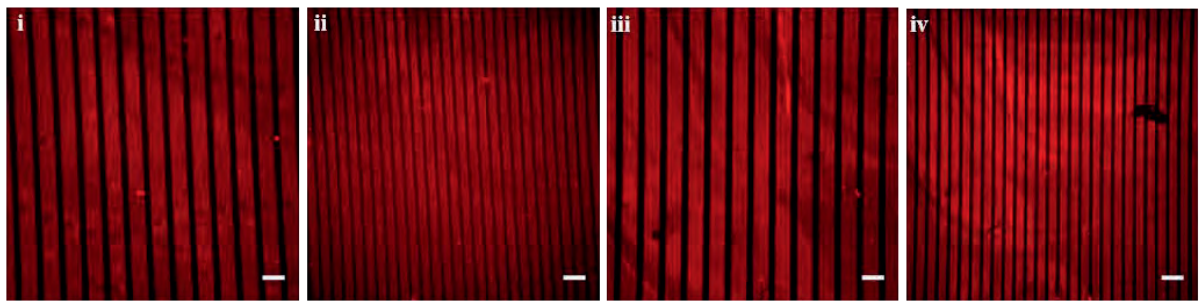

b
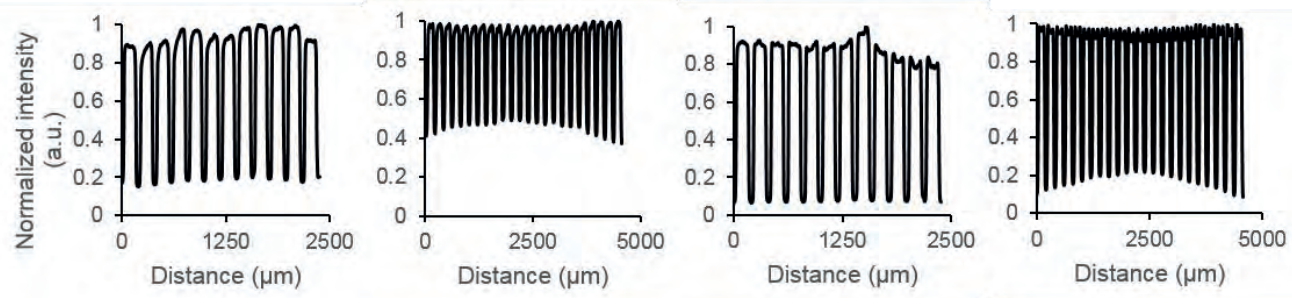

Fig. S6: Correction of Fig. S5 for beam curvature a) Larger overview of experiments from Fig. 2 showing images of Experiment 1 with $5 x$ (i) and 2.5x objective (ii) and Experiment 2 with $5 x$ (iii) and $2.5 x$ objective (iv). Scale bars for $5 \mathrm{x}$ objective images are $200 \mu \mathrm{m}$ and for $2.5 \mathrm{x}$ objective images are $400 \mu \mathrm{m}$. b) Constituent profile plots for images (i)(iv). 

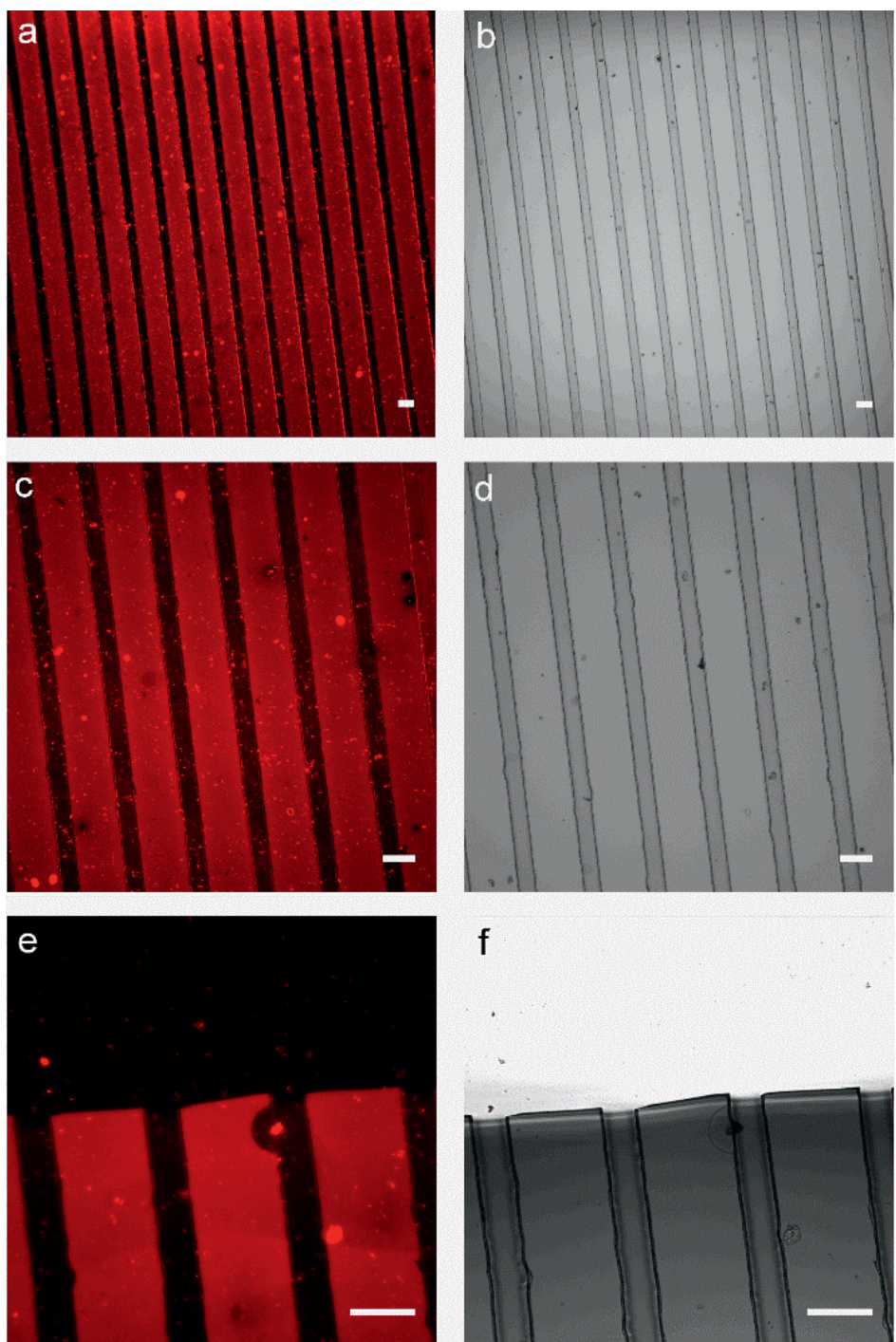

Fig. S7: $\mathrm{P} \mu \mathrm{CP}$ of $\beta-\mathrm{CD}$ surface after $\mathrm{Cy} 5-\mathrm{Ad}_{2}$ immobilization with PDMS stamp still on top of patterned surface. 5x objective image with Cy5 (a) and TL-BF (b) filters. 10x objective images with Cy5 (c) and TL-BF filter (d) filters. 20x objective images with Cy5 (e) and TL-BF filters (f). All scale bars are $100 \mu \mathrm{m}$. 


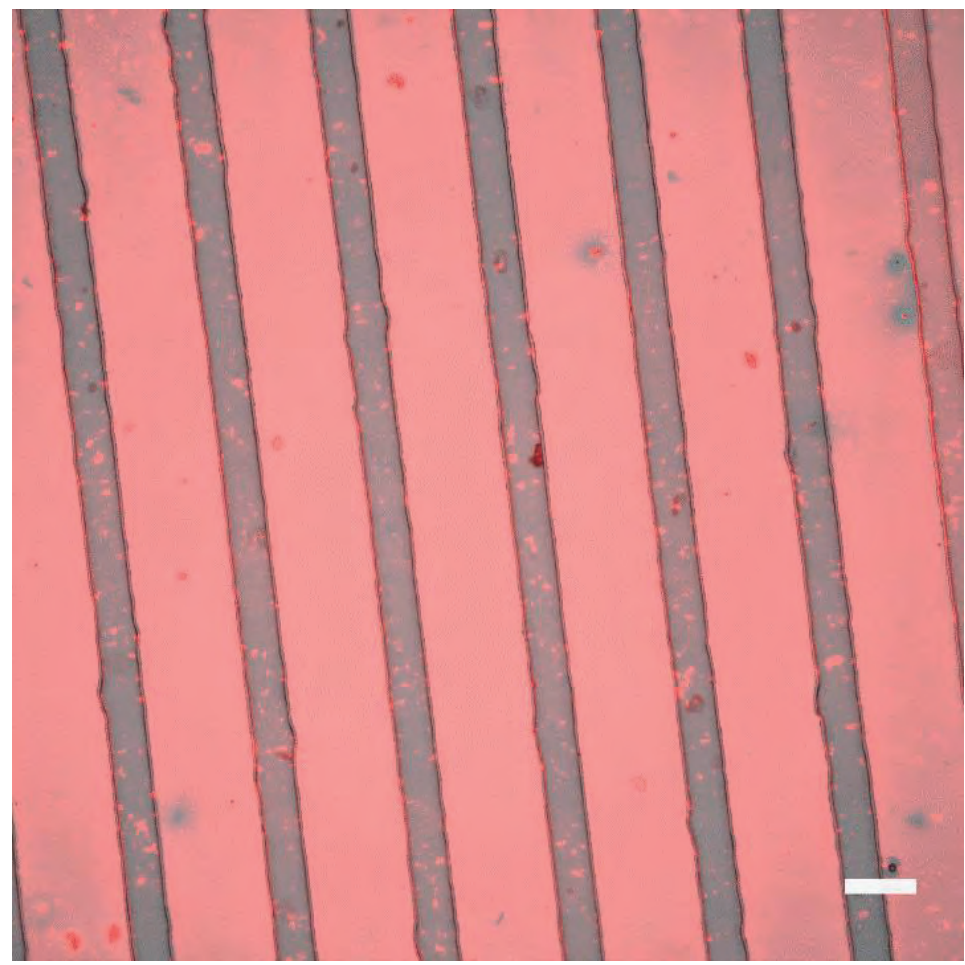

Fig. S8: Overlay of 10x objective images from Fig. S7. Scale bar is $100 \mu \mathrm{m}$. 


\section{Chapter 3}

\section{Plasma Etching for Fabricating Microfluidic Devices with Patterned Monolayers}

Plasma microcontact patterning $(\mathrm{P} \mu \mathrm{CP})$ and replica molding were used to make PDMS/glass microfluidic devices with $\beta$-cyclodextrin $(\beta-C D)$ patterns covalently attached on the glass surface inside a microchannel. Supramolecular reactivity and reusability of the devices were validated through host-guest interactions with diadamantane functionalized Cyanine 5 dye $\left(\mathrm{Cy} 5-\mathrm{Ad}_{2}\right)$. We also show how to fabricate and use patterned microfluidic devices containing multiple microchannels for carrying out more complex analytical experiments. As an example of such an experiment, different concentrations of $\mathrm{Cy} 5-\mathrm{Ad}_{2}$ were flowed separately through the microchannels over one $\beta$-CD patterned surface, which showed a clear increase in fluorescent signal with higher Cy5-Ad2 concentration using optical microscopy. The use of one device/chip for correlation studies, such as (host-guest) binding kinetics, increases experimental control and improves quantitative analysis.

An adjusted version of this chapter is available as a pre-print: Stan B.J. Willems, Jaccoline Zegers, Anton Bunschoten, Martijn Wagterveld, Fijs. W.B. van Leeuwen, Aldrik H. Velders and Vittorio Saggiomo. ChemRxiv, 2019. Manuscript in preparation. 


\subsection{Introduction}

(Bio)sensors using chemically modified surfaces have proven instrumental for the sensitive detection of (bio)analytes. ${ }^{1-6}$ Therefore, the incorporation of modified surfaces within a microfluidic device is becoming an attractive field for creating lab-on-a-chip platforms, e.g. the detection of enzymes on chitosan functionalized surfaces. ${ }^{7}$ Within microfluidic devices, the fabrication of polydimethylsiloxane (PDMS)/glass hybrid systems are highly popular because of their versatility and low-cost. ${ }^{8,9}$ These microfluidic devices allow for facile fluid handling, can analyze small volumes and support direct analyte analysis using microscopy. ${ }^{10}$ Subsequently, analyte quantification defines the final utility of the microfluidic sensor. In light of this, analyte binding to functionalized patterns can be compared with non-functionalized (control) spacing in one field of view in order to quickly establish signal to background ratio's. ${ }^{11}$ Surface patterning of glass surfaces has previously been carried out using techniques such as microcontact printing $(\mu \mathrm{CP}),{ }^{12,13}$ dip-pen nanolithography, ${ }^{14,15}$ and nanoimprint lithography. ${ }^{16,17}$ However, $\mu \mathrm{CP}$ can lack reproducibility, ${ }^{18}$ while dip-pen and nanoimprint lithography are limited by high production costs. Hence, new cost-friendly patterning techniques are in demand, also with respect to analytical fields of research for which (fast) prototyping is required.

The fabrication of PDMS/glass microfluidic devices, also known as 'replica molding', 19 works by activation of the PDMS replica containing micro-sized grooves with a glass surface in the plasma oven, and subsequently applying conformal contact between PDMS and glass surface. The grooves in the PDMS replica form a microchannel with desired size features on the glass surface and the microfluidic device is sealed through covalent bond formation. Importantly, plasma etching technology, in addition to 'cleaning' surfaces by decomposing exposed organic molecules, also creates a layer of reactive silanols on both PDMS and glass surfaces. When the two surfaces are in contact the silanols react to form silyl ethers and covalently 'seal' PDMS to glass, forming a mixed PDMS/glass microfluidic device. ${ }^{20}$ One major drawback of this methodology is that creating patterns of molecules on the glass surface beforehand is not feasible, as the plasma exposure for sealing the microfluidic device will remove the molecular patterns.

We have recently shown that plasma etching, in combination with plasma microcontact patterning $(\mathrm{P} \mu \mathrm{CP})$, can be used to create patterns of covalently bound molecules on glass 
surfaces. ${ }^{21}$ In brief, a PDMS stamp is placed in contact with a covalently modified glass surface and the construct is subjected to plasma etching. Exposed molecules which are not protected by the PDMS stamp are decomposed by plasma, resulting in molecular patterns. It should be noted that the PDMS 'stamp' is now actually functioning as a 'mask': instead of adding molecular patterns to the surface through 'stamping' as with $\mu \mathrm{CP}$, patterns are now generated by removing molecular functionality through plasma etching using the same PDMS stamp. Moreover, plasma exposed areas between the stamp patterns and outside the stamp area now contain reactive silanols which can be used for covalent bonding with PDMS surfaces. Based on this utility, we speculated that in order to create a microfluidic device with embedded covalently functionalized monolayer patterns, $\mathrm{P} \mu \mathrm{CP}$ could be combined with PDMS replica molding. Previously, this concept has only been carried out with surfaces that contain patterns of physisorbed proteins within microfluidic channels. ${ }^{22,23}$

As proof of concept for combined $\mathrm{P} \mu \mathrm{CP} /$ replica molding with a covalent modified surface, we investigated the fabrication of $\beta$-cyclodextrin $(\beta-C D)$ patterns within a microfluidic device (Fig. 3.1). Cyclodextrins are well known for their applications in food technology, pharmaceutics and drug delivery, but have also emerged as versatile platforms for material science through their interactions with metal ions and inorganic nanoparticles. ${ }^{24}$ Through hostguest chemistry, the inside of $\beta$-CD's hydrophobic cavity (host) can be used for immobilization of guest functionalized molecules, such as Cyanine 5-diadamantane ( $\left.\mathrm{Cy} 5-\mathrm{Ad}_{2}\right),{ }^{25}$ and recently for capturing adamantane functionalized microparticles on flow. ${ }^{26}$ Therefore, we validated the functionalization of $\beta-C D$ patterns on the glass surface within the fabricated microfluidic device through addition of $\mathrm{Cy} 5-\mathrm{Ad}_{2}$, which allowed us to use fluorescence microscopy to study the immobilization and release of $\mathrm{Cy} 5-\mathrm{Ad}_{2}$ under flow. 
a

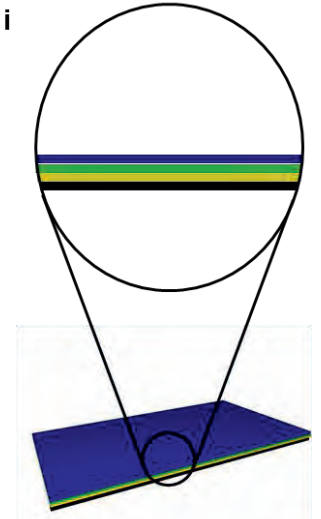

Covalently

functionalized $\beta-C D$

surface

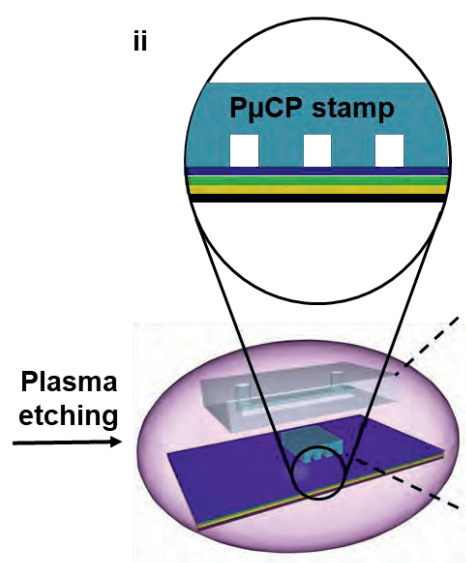

$\mathrm{P} \mu \mathrm{CP}$ and activation of unprotected glass surface and PDMS replica

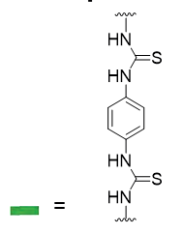

PDITC

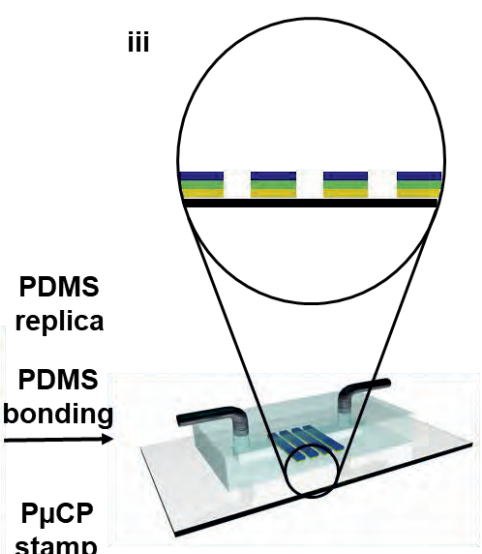

Patterned microfluidic

iii

stamp

\section{device}

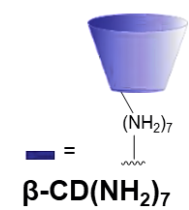

b

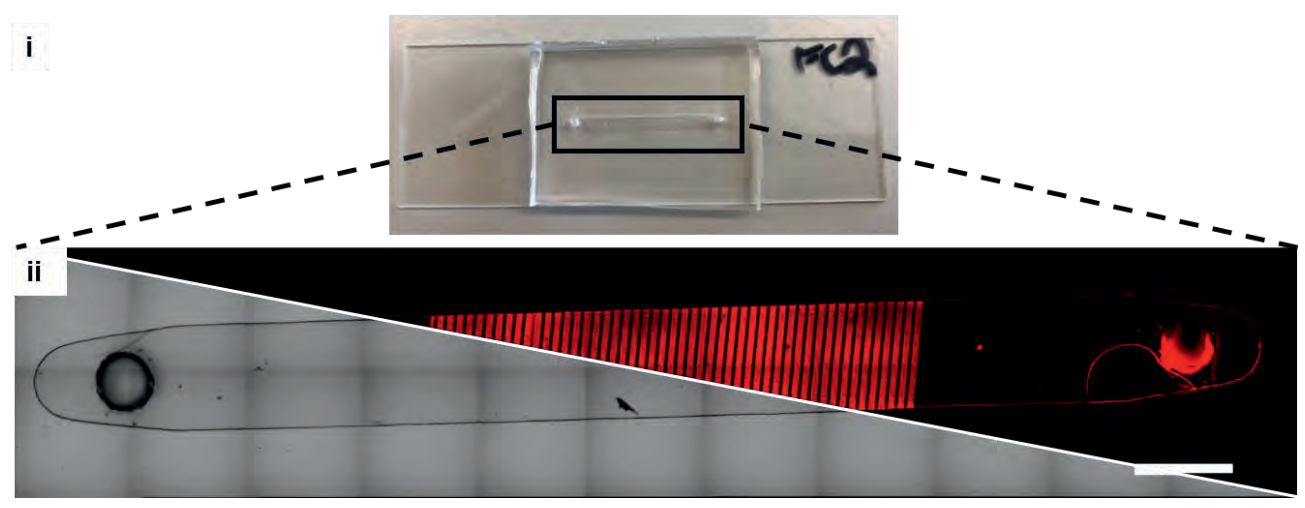

Fig. 3.1: a) Scheme showing the fabrication of the patterned microfluidic device: a fully functionalized $\beta$-CD surface is obtained after silanization with 3-aminopropyltriethoxysilane (APTES) and 1,4-phenelynediisothiocyanate (PDITC) cross linking of heptakis amino $\beta-C D(i)$, then a PDMS stamp with line features is placed on top of modified surface for $\mathrm{P} \mu \mathrm{CP}$ and plasma etched together with the PDMS replica (ii), and finally the PDMS replica is chemically bonded to the patterned $\beta$-CD surface via plasma bonding to create a $\beta$-CD patterned microfluidic device (iii). b) Photograph of a single channel patterned microfluidic device (i) and microscope images of channel using $5 x$ objective and $11 \times 2$ tile scan after addition of Cy5-Ad2 using the Brightfield (lower left) and Cy5-fluorescence (upper right) settings (ii) (scale bar is $2 \mathrm{~mm}$ ). 


\subsection{Experimental Section}

\section{General}

Chemicals were purchased from Sigma Aldrich/Merck unless mentioned otherwise. Menzel-Gläser borosilicate glass microscope slides were used to fabricate the microfluidic device and were purchased from Thermo Scientific. Hyper pure polished silicon wafers (76.2 mm diameter, University Wafer, USA) were used as silicon masters. A photomask template containing patterns, with different feature and spacing sizes, was designed and ordered from JD photo data (UK). Deionized (DI) water was used in all the experiments. Cyanine 5diadamantane ( $\left.\mathrm{Cy} 5-\mathrm{Ad}_{2}\right)$ was synthesized by Dr. Mark Rood from the Interventional Molecular Imaging lab at the Leiden University Medical Centre (LUMC, Leiden, the Netherlands). ${ }^{25}$

\section{Instrumentation}

For the preparation of the silicon master for PDMS stamps, a standard UV-lithography set-up was used. $50 \mu \mathrm{m}$ thick SU-8 2025 negative photoresist on the silicon wafer was irradiated with a UV lamp at $\lambda=350 \mathrm{~nm}$ at 50\% intensity. An Inseto Plasma Etch, Inc. PE-25 benchtop air plasma cleaner was used for plasma cycles of $1 \mathrm{~min}$ at its maximum RF plasma power of $100 \mathrm{~W}$ with an air flow of $\sim 10 \mathrm{cc} / \mathrm{min}$, which allowed for a vacuum pressure of 200-250 mTorr within the chamber during plasma treatment. An RC6 Chemistry Hybrid Pump from Vacuubrand (vacuum of $2 \times 10^{-3}$ mbar) was used for applying high vacuum condition in desiccators. The functionalized glass surfaces were imaged in air, using a Leica DMi8 epifluorescence microscope with 10X or 5X magnification objective lens and beam intensity of $100 \%$. For $10 \mathrm{X}$ objective images, exposure time was set to $278 \mathrm{~ms}$ and for $5 \mathrm{X}$ objective images to $500 \mathrm{~ms}$. Cy5-Ad2 was excited at a wavelength $\left(\lambda_{\mathrm{ex}}\right)$ of $590-650 \mathrm{~nm}$ and emission $\left(\lambda_{\mathrm{em}}\right)$ was collected at $662-738 \mathrm{~nm}$ (using the Y5 filter cube).

\section{FIJI/ImageJ data analysis}

The results were analysed with FIJI software (ImageJ). ${ }^{27}$ The images were rotated in such a way that the patterns were positioned vertically and the gray value intensity range was set to 0-3000 a.u. Then, a square was drawn over the patterns and the averaged gray values were collected within that square. The gray values (a.u.) were then plotted against the distance $(\mu \mathrm{m})$ 
to make a profile plot of the image. For calculating normalized fluorescent intensity of patterns and spacing, the raw values were normalized to the minimal value of the data set. For creating a live profile plot video in FIJI software, a script was obtained from image.sc forum (https://forum.image.sc/t/save-real-time-plot/7237) posted and created by Nicolas Chiaruttini aka 'NicoKiaru' (Script S1). ${ }^{28}$ The sequence of images from the time lapse experiment was opened and the maximum brightness was adjusted to 3000 a.u. (gray value). A 'live' profile plot was created as before by plotting the gray values against the distance, but also selecting live on the profile plot window. The range for the y-axis (gray values) was then set to a maximum value of 3000 a.u.. The script S1 was then copied into the 'Script' function (from main menu File $\rightarrow$ New) and then run with the opened sequence of images and the live profile plot. This creates a video of the profile plot window which was then combined 'vertically' with the sequence of images using the 'Combine' function (from main menu Image -> Stacks -> Tools). The video was saved at 7 frames per second.

\section{Glass surface functionalization}

The general glass surface functionalization with $\beta$-cyclodextrin was performed as described by Onclin et al. ${ }^{29}$ In short, glass microscope slides of size 76 x $26 \mathrm{~mm}$ were cleaned and oxidized with piranha solution $\left(\mathrm{H}_{2} \mathrm{SO}_{4}(95-98 \%) / \mathrm{H}_{2} \mathrm{O}_{2}(35 \%)\right.$, 3.33:1 v/v; Warning! Piranha solutions must be handled with caution as they may unexpectedly detonate) for 45 minutes, rinsed with large amounts DI water, and dried under $\mathrm{N}_{2}$. The glass slides were placed in a high vacuum, pre-heated desiccator together with a glass vial containing $1 \mathrm{~mL}$ of 3aminopropyltriethoxysilane (APTES, 99\%) and placed in an oven at $70{ }^{\circ} \mathrm{C}$ overnight for chemical vapor deposition (CVD) of APTES. Following amine monolayer formation, the glass slides were removed from the desiccator and rinsed with toluene (HPLC grade, VWR, the Netherlands) and dichloromethane (DCM, VWR). The glass slides were then cured for at least 1 hour in the oven at $70^{\circ} \mathrm{C}$. Next, the glass slides were immersed in $0.1 \mathrm{M} \mathrm{1,4-phenylene}$ diisothiocyanate (PDITC, TCI Chemicals Belgium) in anhydrous toluene ( $\max 0.002 \% \mathrm{H}_{2} \mathrm{O}$, VWR) for 2 hours under argon atmosphere to yield isothiocyanate bearing layers. Following the immersion, the surfaces were rinsed with toluene and DCM and incubated in $0.72 \mathrm{mM}$ heptakis amino $\beta$-cyclodextrin ( $\beta-\mathrm{CD}$, Cyclodextrin Shop, the Netherlands) in aqueous solution at $\mathrm{pH} 8.0$ (reached by adding small amounts of $1 \mathrm{M} \mathrm{NaOH}$ ). This incubation was carried out for at least 2 hours. Surfaces were then rinsed with DI water and dried with nitrogen. 


\section{PDMS replica fabrication (PDMS microchannel features)}

A 3D positive channel with a channel of dimensions of $20 \times 2 \times 0.5 \mathrm{~mm}$ was designed in SketchUP (Trimble) and printed with an LCD 3D printer (Photon Anycubic). For the microfluidic device containing 5 channels, the same channel dimensions of $20 \times 2 \times 0.5 \mathrm{~mm}$ were used with a spacing of $2 \mathrm{~mm}$ between the channels. For an easy peeling of the PDMS, the $3 \mathrm{D}$ printed molds were coated with trichloro $(1 \mathrm{H}, 1 \mathrm{H}, 2 \mathrm{H}, 2 \mathrm{H}$-perfluorooctyl)silane (PFOTS, 97\%) using chemical vapor deposition (CVD) in a vacuum desiccator; The 3D printed structure was air plasma activated for 30s, then it was placed in a dessicator with a vial of $100 \mu \mathrm{L}$ of PFOTS. The desiccator was then left under static vacuum for overnight CVD of PFOTS. After deposition in the desiccator, the 3D printed structure was removed from the desiccator and left in the oven at $70{ }^{\circ} \mathrm{C}$ for $1 \mathrm{~h}$, then it was washed with ethanol and isopropanol. PDMS replicas were prepared by pouring PDMS mixture (10:1 PDMS/curing agent) on the 3D printed PFOTS coated mold. After overnight curing at $70^{\circ} \mathrm{C}$, the PDMS was cut out the mold and sonicated in ethanol for 4 minutes for removing the small molecular weight and unreacted PDMS chains. The PDMS replica was then dried with nitrogen. An inlet and outlet were then created on the edges of the microchannel features by puncturing through the PDMS with a $1.5 \mathrm{~mm} \varnothing$ punch (KAI, Japan).

\section{Plasma microcontact patterning $(\mathrm{P} \mu \mathrm{CP})$}

Creating patterns was carried out through $\mathrm{P} \mu \mathrm{CP}$ as reported previously. ${ }^{21}$ Shortly, PDMS stamps for $\mathrm{P} \mu \mathrm{CP}$ were fabricated using the standard technique from Whitesides. ${ }^{13} \mathrm{~A}$ silicon master was first fabricated by spin-coating at $1500 \mathrm{rpm}$ with SU-8 2025 photoresist (Microchem, USA) to yield a $50 \mu \mathrm{m}$ thick photoresist layer and then treated using UV photolithography with a photomask containing $150 \mu \mathrm{m}$ patterns and $50 \mu \mathrm{m}$ pattern spacing. The silicon master and a separate glass vial containing $100 \mu \mathrm{L}$ of PFOTS were introduced into a desiccator under vacuum for overnight CVD. After incubation with PFOTS, the wafer was cleaned with isopropanol and dried with nitrogen. Stamps were prepared by casting a 10:1 (w/w) mixture of poly(dimethylsiloxane) (PDMS) and curing agent (Sylgard 184, Dow Corning) onto the silicon master with $150 \mu \mathrm{m}$ patterns and $50 \mu \mathrm{m}$ pattern spacing. After overnight curing at $70{ }^{\circ} \mathrm{C}$, the PDMS stamps were cut out the master to ca $0.75 \mathrm{~cm}^{2}$ and sonicated in ethanol to remove low molecular weight PDMS. The PDMS stamps were then brought into conformal contact with the freshly prepared functionalized glass surfaces. $\beta-C D$ 
covered slides were then placed in the plasma cleaner (using the settings mentioned in the instrumentation section) for 4 cycles of 1 minute each.

\section{Microfluidic channel fabrication}

The PDMS replica was placed facing upwards in the plasma oven along with the $\mathrm{P} \mu \mathrm{CP}$ $\beta$-CD surface for the $4^{\text {th }}$ plasma cycle. The PDMS stamp was removed from the patterned $\beta$ CD surface and the PDMS replica was firmly placed on top of the patterned glass slide, with the microchannel facing downwards. A piece of paper was then placed on top of the PDMS replica, followed by a non-functionalized microscope glass slide. Clamps were then used to keep the non-functionalized microscope glass slide and PDMS replica firmly pressed against the functionalized glass surface (as shown in Figure S2) and microfluidic device was incubated in the oven at $70^{\circ} \mathrm{C}$ for 1 hour. The holders were then removed from the microfluidic device and tubing was connected to the inlet and outlet of the channel. The inlet tubing was then connected to a syringe pump and the outlet tubing to a waste vial.

\section{Flow experiments}

Before using the $\beta$-CD functionalized microfluidic device for immobilization of Cy5$\mathrm{Ad}_{2}$, the channel and surface was thoroughly washed with DI water at $800 \mu \mathrm{L} / \mathrm{min}$ for $15 \mathrm{mins}$ in order to clean the channel before use. Then, $0.23 \mu \mathrm{M}$ of $\mathrm{Cy} 5-\mathrm{Ad}_{2}$ in phosphate buffered saline (PBS: $10 \mathrm{mM} \mathrm{Na} 2 \mathrm{HPO}_{4}, 1.8 \mathrm{mM} \mathrm{KH}_{2} \mathrm{PO}_{4}, 137 \mathrm{mM} \mathrm{NaCl}, 2.7 \mathrm{mM} \mathrm{KCl}, \mathrm{pH}$ 7.4) was flowed over $\beta$-CD functionalized microfluidic device at a flow rate of $50 \mu \mathrm{L} / \mathrm{min}$ for $15 \mathrm{mins}$. The surface was imaged using the Cy5 filter on the microscope before and during addition. After addition of Cy5-Ad2, the surface was first washed with a flow of PBS at $200 \mu \mathrm{L} / \mathrm{min}$ for $5 \mathrm{mins}$ and then DI water also at $200 \mu \mathrm{L} / \mathrm{min}$ and for $5 \mathrm{mins}$. Again, images of the surface were captured with Cy5 filter on the microscope. To remove immobilized $\mathrm{Cy} 5-\mathrm{Ad} 2$, the surface was washed for several cycles with an aqueous solution of $10 \mathrm{mM} \beta-\mathrm{CD}$, pH 10 at $800 \mu \mathrm{L} / \mathrm{min}$ for 10 mins per cycle. When Cy5-Ad 2 was sufficiently removed from the surface according to fluorescence imaging, the microfluidic device was washed with a flow of DI water at 200 $\mu \mathrm{L} / \mathrm{min}$ for 5 mins to remove remaining $\beta$-CD from the channel and surface. Then, a flow of Cy5-Ad2 was re-applied at the same flow rate and duration, and the surface was washed with a flow PBS and DI water. The re-usability of the $\beta$-CD functionalized microfluidic device was repeated for a total of 4 uses. For determining the binding kinetics of $\mathrm{Cy} 5-\mathrm{Ad}_{2}$ to $\beta-\mathrm{CD}$, a $5 \mathrm{x}$ 
serial dilution of $0.23 \mu \mathrm{M}$ Cy5- $\mathrm{Ad}_{2}$ was first obtained by diluting from a concentration of 0.23 $\mu \mathrm{M}$ until $0.36 \mathrm{nM}$ in PBS. The different concentrations of Cy5-Ad 2 were sequentially flowed over different channels in the multi-channel microfluidic devices at a flow rate of $50 \mu \mathrm{L} / \mathrm{min}$ for 15 mins (then washed with PBS at $200 \mu \mathrm{L} / \mathrm{min}$ for 5 mins and DI water at $200 \mu \mathrm{L} / \mathrm{min}$ for 5 mins). 


\subsection{Results and Discussion}

Fig. 3.1a illustrates the steps required for microfluidic device fabrication. A fully functionalized $\beta-C D$ surface was first obtained following a literature protocol from Onclin et $a l .{ }^{29}$ (Fig. 3.1a(i)): the glass functionalization with heptakis amino $\beta-\mathrm{CD}$ was carried out using 3-aminopropyltriethoxysilane (APTES) for obtaining an amine functionalized surface, 1,4phenylenediisothiocyanate (PDITC) for creating an amine reactive surface, and finally incubation of heptakis amino $\beta-C D$. Surface patterning of $\beta-C D$ was realized via $\mathrm{P} \mu \mathrm{CP}$ with a PDMS stamp (150 $\mu \mathrm{m}$ line features and $50 \mu \mathrm{m}$ spacing; four plasma cycles of $1 \mathrm{~min}),{ }^{21,30}$ also generating a reactive surface for subsequent microchannel fabrication (Fig. 3.1a(ii)). In parallel, PDMS was poured into a 3D printed mould containing $20 \times 2 \times 0.5 \mathrm{~mm}$ (length $\mathrm{x}$ width $\mathrm{x}$ height) groove features, forming the PDMS replica after curing in the oven. The PDMS replica was activated in the plasma oven along with the stamp protected patterns for the final plasma cycle (Fig. 3.1a(ii)). Following the plasma treatment, the activated PDMS replica was then placed in conformal contact with the patterned $\beta-\mathrm{CD}$ glass surface to chemically attach the PDMS to the glass surface and create a microfluidic channel over the patterned area (Fig. 3.1a(iii)). In order to promote homogenous sealing of the microfluidic device, we ensured conformal contact between the PDMS replica and glass surface, then attached clamps to keep the microchannel firmly pressed against the glass surface which was followed by further bonding in the oven (Fig. S1). The resulting microfluidic device is depicted in Fig. 3.1b.

Confirmation of $\beta$-CD pattern formation was realized by flowing a $0.23 \mu \mathrm{M}$ solution of Cy5-Ad2 through the microfluidic device for $15 \mathrm{~min}$ at $50 \mu \mathrm{L} / \mathrm{min}$. Excess Cy5-Ad2 was washed away with PBS and DI water, resulting in clear $150 \mu \mathrm{m}$ broad line patterns when analyzed with a fluorescence microscope (Fig. 3.1b(ii)). A more detailed sequence of events is depicted in Fig. S2 showing the microscope images of surface before Cy5-Ad2 addition, during addition and after washing away excess Cy5-Ad 2 with PBS and DI water. After washing away excess Cy5- $\mathrm{Ad}_{2}$, the fluorescence intensity of remaining $\mathrm{Cy} 5-\mathrm{Ad}_{2}$ on $\beta-\mathrm{CD}$ is generally c.a. 4 times higher than the background signal between patterns, which is a result of the high affinity divalent host-guest interactions between $\mathrm{Cy} 5-\mathrm{Ad}_{2}$ and $\beta-\mathrm{CD}$ (Fig. S2b(ii)). 
An important characteristic of (bio)sensors incorporated within simple microfluidic devices is re-usability of the sensor in order to run different analytical experiments over the same sensor. In our case, supramolecular host-guest chemistry of $\beta$-CD with adamantane guest molecules allows for non-covalent, reversible interactions. ${ }^{31}$ Hence, stripping the surface of immobilized $\mathrm{Cy} 5-\mathrm{Ad}_{2}$ analytes was also studied using a known procedure based on removal of diadamantane functionalized guest molecules with $10 \mathrm{mM} \beta-\mathrm{CD},{ }^{32}$ which was carried out using a flow rate of $800 \mu \mathrm{L} / \mathrm{min}$. In Fig. 3.2a, the $1^{\text {st }}$ removal of Cy5- $\mathrm{Ad}_{2}$ and subsequent repeat addition and removal are shown. From the profile plots it should be noted that the intensity values were normalized for the minimal values within the graph (normalizing the spacing intensity to 1). Fig. 3.2b illustrates that the printed surface was tested for a total of 4 additions of $\mathrm{Cy} 5-\mathrm{Ad}_{2}$ and that the loading-rate diminishes by approximately $10 \%$ in the consecutive recycling steps, but the pattern intensity remains preserved at c.a. 3.5. The washing step with $\beta-\mathrm{CD}$ does not completely remove immobilized Cy5-Ad2 (Fig. 3.2a(ii)+(iv)). However, the

a
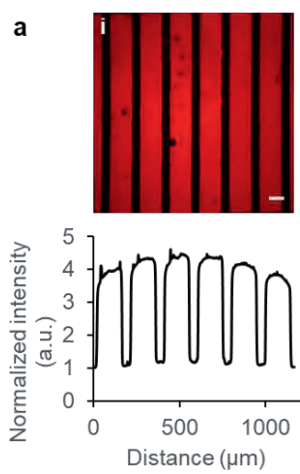

b
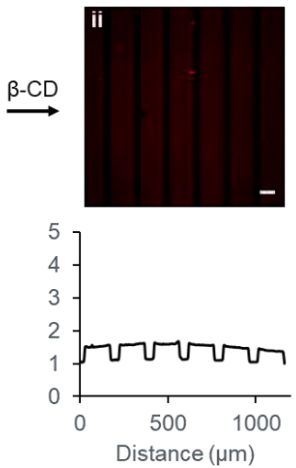

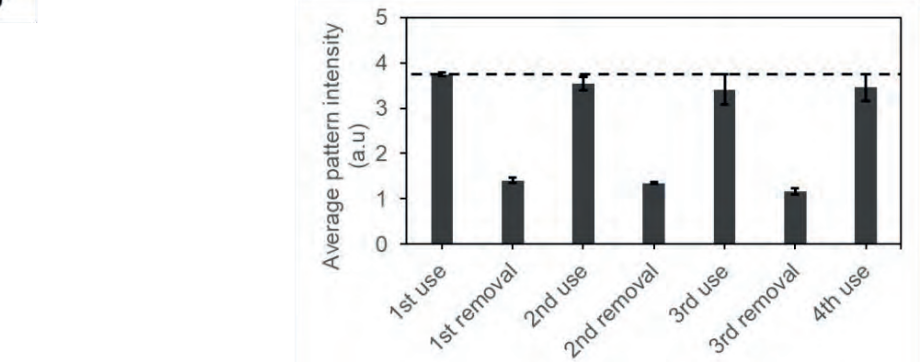

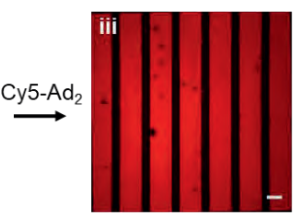
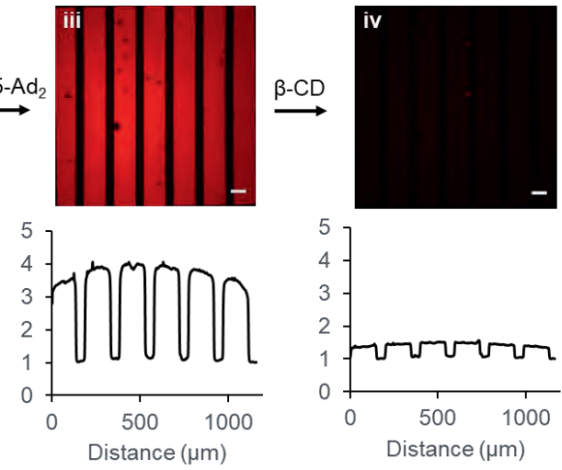

Fig. 3.2: a) Re-usability of microfluidic devices showing $\beta-C D$ patterned surface after addition and washing away excess Cy5-Ad 2 (i), after removal of immobilized Cy5-Ad 2 by washing with $\beta-C D$ solution (ii), after $2^{\text {nd }}$ addition and washing away excess $\mathrm{Cy} 5-\mathrm{Ad}_{2}$ (iii), and after $2^{\text {nd }}$ removal of immobilized Cy5- $\mathrm{Ad}_{2}$ by washing with $\beta-\mathrm{CD}$ solution (iv). Images were taken at the same location to allow for fair comparison and were captured with $10 \mathrm{x}$ objective. Scale bars are $100 \mu \mathrm{m}$. The profile plots below the images show the normalized intensity of patterns and spacing vs the distance. b) Graph showing the average pattern intensity for 4 uses/additions of $\mathrm{Cy} 5$ - $\mathrm{Ad}_{2}$ and 3 removals with $\beta$-CD. 
average pattern intensity after removal remains approximately between 1-1.5 for all removal steps which indicates reversibility is reproducible (Fig. 3.2b). The sensor technology presented holds potential for monitoring extraction and release of analytes through supramolecular interactions for guest molecules which have similar binding affinities as $\mathrm{Cy} 5-\mathrm{Ad}_{2}$ (approximately between $10^{7}-10^{10} \mathrm{M}^{-1}$ in aqueous solutions). ${ }^{25,33}$

Within lab-on-a-chip technology, the fabrication of (bio)arrays for carrying out multiple analyses using one chip is quite popular. This is also particularly useful for analyzing binding kinetics of analytes through varying analyte concentration. In this case, using one surface increases the accuracy of the quantitative analysis with techniques such as fluorescence microscopy. To demonstrate the utility of our microfluidic device in such a test, we exchanged the single channel PDMS device for a multiple channel one containing 5 channels with the same groove dimensions as used before. The $\mathrm{P} \mu \mathrm{CP}$ step on $\beta$-CD functionalized surfaces was slightly modified to incorporate $\beta-\mathrm{CD}$ patterns within all 5 channels: before plasma etching, $\mathrm{P} \mu \mathrm{CP}$

a

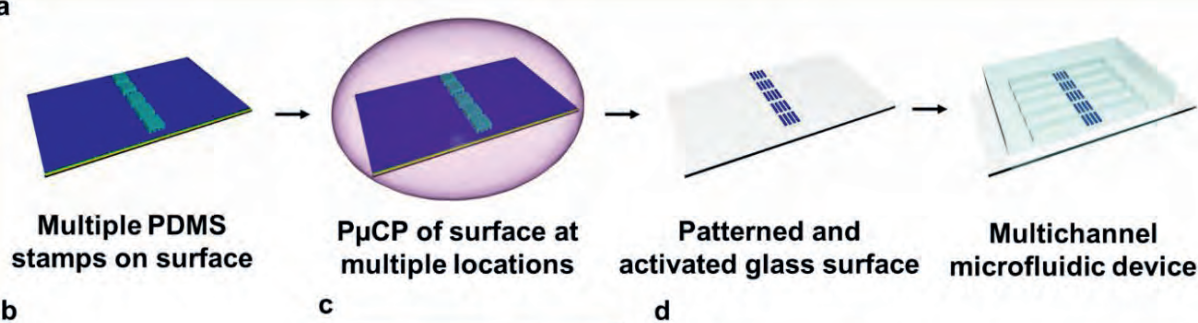

b
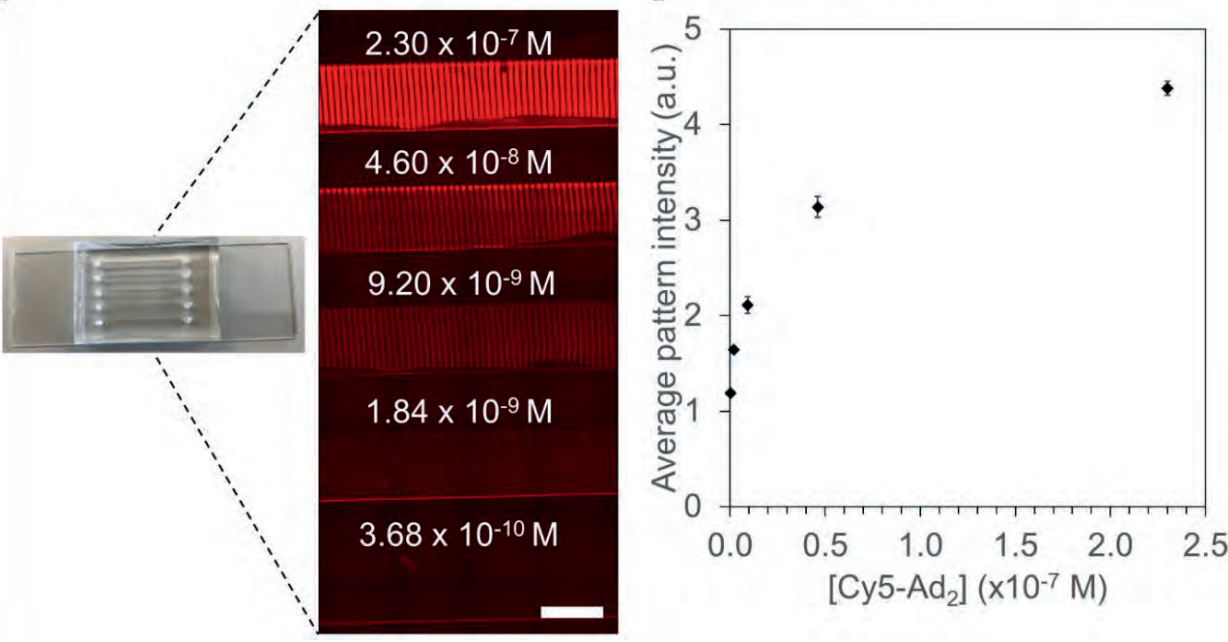

Fig. 3.3: a) Scheme explaining fabrication of multichannel microfluidic device with $\beta$-CD patterns b) Photograph of patterned microfluidic device with 5 channels. c) Microscope image of area depicted in a) after addition of different concentrations of $\mathrm{Cy} 5-\mathrm{Ad}_{2}$. The concentration used is written above the designated microchannel. The image was captured using a $5 \mathrm{x}$ objective and $8 \times 4$ tilescan. Scale bar is $2 \mathrm{~mm}$. d) Graph showing the average pattern intensity obtained with the different concentrations of Cy5- $\mathrm{Ad}_{2}$ used. 
stamps were cut in 5 pieces, with each piece having approximately the same width of one microchannel, and then placed on the $\beta$-CD glass surface (Fig. 3.3a). At this stage it is important that sufficient space (approximately $2 \mathrm{~mm}$ ) is left between the $\mathrm{P} \mu \mathrm{CP}$ stamps and that the stamps are placed within the area where the microchannels will be formed when bonding the PDMS replica. Otherwise, resulting patterns of $\beta-C D$ will overlap neighboring channels, which hinders proper sealing of the PDMS replica between channels and causes leaching of solution from one channel to another. A $5 \mathrm{x}$ serial dilution of a $\mathrm{Cy} 5-\mathrm{Ad}_{2}$ solution was then made and flowed over the $\beta$-CD patterned microfluidic device with multiple channels (Fig. 3.3b), starting from the highest concentration (top channel) to the lowest concentration (bottom channel) (Fig. 3.3c). The average pattern intensity in Fig. 3.3d was determined by capturing images of each microchannel using the 10x objective and normalizing profile plots for the minimal values, i.e. spacing between $\beta$-CD patterns. Moreover, a control experiment with $0.23 \mu \mathrm{M}$ of $\mathrm{Cy} 5-\mathrm{Ad}_{2}$ was carried out to show that using multiple strips of PDMS stamps during the P $\mu \mathrm{CP}$ step does not significantly alter the density of $\beta$-CD units over the different patterned areas on one surface (Fig. S3). As expected, the fluorescence increases when higher concentrations of $\mathrm{Cy} 5-\mathrm{Ad}_{2}$ are used, and the trend in the graph shows a typical binding affinity profile (Fig. 3.3d). These results show that these patterned microfluidic devices can also be used for carrying out analysis of different concentrations of analytes and suggest that they could also be used for comparing binding affinity of different analytes on one surface. 


\subsection{Conclusion}

In this chapter, we have shown how to fabricate microfluidic devices containing covalently functionalized molecular patterns by combining $\mathrm{P} \mu \mathrm{CP}$ and replica molding. Incorporation of the patterned surfaces in the microfluidic device allows for simple fluid handling and approaching sensor applications within aqueous flow streams. As a proof of concept for sensing applications, we studied the reversible host-guest interactions between patterns of $\beta-\mathrm{CD}$, included within the microfluidic device, and $\mathrm{Cy} 5-\mathrm{Ad} 2$. The immobilization and removal of $\mathrm{Cy} 5-\mathrm{Ad}_{2}$ was easily analyzed using a fluorescence microscope and it was possible to re-use the $\beta$-CD patterned microfluidic device several times, which is beneficial for extraction applications and avoids laborious repetition of the surface functionalization. It is also possible to study binding of several analytes on one surface through creating a microfluidic device with multiple channels, all containing molecular patterns. Through this enabling technology for fabrication of patterned PDMS/glass hybrid microfluidic devices, a way is opened for researchers less experienced in surface functionalization and working on different fields ranging from (bio) sensing applications, such as immobilization of micron-sized entities, ${ }^{26}$ to analytical chemistry and medical applications like lab- or organs-on-a-chip. ${ }^{34,35}$ 


\subsection{References}

1. Kumar, A.; Biebuyck, H. A.; Whitesides, G. M. Patterning Self-Assembled Monolayers: Applications in Materials Science. Langmuir 1994, 10 (5), 1498-1511.

2. Mrksich, M.; Whitesides, G. M. Using Self-Assembled Monolayers to Understand the Interactions of Man-made Surfaces with Proteins and Cells. Annual Review of Biophysics and Biomolecular Structure 1996, 25 (1), 55-78.

3. Hsu, S.-H.; Yilmaz, M. D.; Blum, C.; Subramaniam, V.; Reinhoudt, D. N.; Velders, A. H.; Huskens, J. Expression of Sensitized Eu3+ Luminescence at a Multivalent Interface. Journal of the American Chemical Society 2009, 131 (35), 12567-12569.

4. Dorokhin, D.; Hsu, S.-H.; Tomczak, N.; Reinhoudt, D. N.; Huskens, J.; Velders, A. H.; Vancso, G. J. Fabrication and Luminescence of Designer Surface Patterns with $\beta$-Cyclodextrin Functionalized Quantum Dots via Multivalent Supramolecular Coupling. ACS Nano 2010, 4 (1), 137-142.

5. Gonzalez-Campo, A.; Hsu, S. H.; Puig, L.; Huskens, J.; Reinhoudt, D. N.; Velders, A. H. Orthogonal covalent and noncovalent functionalization of cyclodextrin-alkyne patterned surfaces. Journal of the American Chemical Society 2010, 132 (33), 11434-11436.

6. de laRica, R.; Fratila, R. M.; Szarpak, A.; Huskens, J.; Velders, A. H. Multivalent Nanoparticle Networks as Ultrasensitive Enzyme Sensors. Angewandte Chemie International Edition 2011, 50 (25), 5704-5707.

7. Wang, Z.; He, L.; Lv, J.; Kimura, M. Electrodeposition of thin chitosan membrane in freestanding SU-8 microfluidic channel for molecular addressing by capillary effect. Materials Research Express 2019, 6 (4), 045403.

8. McDonald, J. C.; Whitesides, G. M. Poly (dimethylsiloxane) as a material for fabricating microfluidic devices. Accounts of Chemical Research 2002, 35 (7), 491-499.

9. Ren, K.; Zhou, J.; Wu, H. Materials for microfluidic chip fabrication. Accounts of Chemical Research 2013, 46 (11), 2396-2406.

10. Lafleur, J. P.; Jönsson, A.; Senkbeil, S.; Kutter, J. P. Recent advances in lab-on-a-chip for biosensing applications. Biosensors and Bioelectronics 2016, 76, 213-233.

11. Kane, R. S.; Takayama, S.; Ostuni, E.; Ingber, D. E.; Whitesides, G. M. Patterning proteins and cells using soft lithography. In The Biomaterials: Silver Jubilee Compendium, Williams, D. F., Ed. Elsevier Science: Oxford, 1999; pp 161-174.

12. Xia, Y.; Mrksich, M.; Kim, E.; Whitesides, G. M. Microcontact Printing of Octadecylsiloxane on the Surface of Silicon Dioxide and Its Application in Microfabrication. Journal of the American Chemical Society 1995, 117 (37), 9576-9577.

13. Xia, Y.; Whitesides, G. M. Soft Lithography. Angewandte Chemie International Edition 1998, 37 (5), 550-575.

14. Piner, R. D.; Zhu, J.; Xu, F.; Hong, S.; Mirkin, C. A. 'Dip-Pen' Nanolithography. Science 1999, 283 (5402), 661-663.

15. Wu, C.-C.; Reinhoudt, D. N.; Otto, C.; Subramaniam, V.; Velders, A. H. Strategies for Patterning Biomolecules with Dip-Pen Nanolithography. Small 2011, 7 (8), 989-1002.

16. Zankovych, S.; Hoffmann, T.; Seekamp, J.; Bruch, J. U.; Torres, C. M. S. Nanoimprint lithography: challenges and prospects. Nanotechnology 2001, 12 (2), 91-95.

17. Guo, L. J. Nanoimprint Lithography: Methods and Material Requirements. Advanced Materials 2007, 19 (4), 495-513.

18. Perl, A.; Reinhoudt, D. N.; Huskens, J. Microcontact Printing: Limitations and Achievements. Advanced Materials 2009, 21 (22), 2257-2268. 
19. Xia, Y.; McClelland, J. J.; Gupta, R.; Qin, D.; Zhao, X.-M.; Sohn, L. L.; Celotta, R. J.; Whitesides, G. M. Replica molding using polymeric materials: A practical step toward nanomanufacturing. Advanced Materials 1997, 9 (2), 147-149.

20. Duffy, D. C.; McDonald, J. C.; Schueller, O. J. A.; Whitesides, G. M. Rapid Prototyping of Microfluidic Systems in Poly(dimethylsiloxane). Analytical Chemistry 1998, 70 (23), 49744984.

21. Willems, S. B. J.; Schijven, L. M. I.; Bunschoten, A.; van Leeuwen, F. W. B.; Velders, A. H.; Saggiomo, V. Covalently bound monolayer patterns obtained by plasma etching on glass surfaces. Chemical Communications 2019, 55 (53), 7667-7670.

22. Rhee, S. W.; Taylor, A. M.; Tu, C. H.; Cribbs, D. H.; Cotman, C. W.; Jeon, N. L. Patterned cell culture inside microfluidic devices. Lab on a Chip 2005, 5 (1), 102-107.

23. Rosso, M.; van Steijn, V.; de Smet, L. C. P. M.; Sudhölter, E. J. R.; Kleijn, C. R.; Kreutzer, M. T. Reaction-diffusion analysis for one-step plasma etching and bonding of microfluidic devices. Applied Physics Letters 2011, 98 (17), 174102.

24. Prochowicz, D.; Kornowicz, A.; Lewiński, J. Interactions of Native Cyclodextrins with Metal Ions and Inorganic Nanoparticles: Fertile Landscape for Chemistry and Materials Science. Chemical Reviews 2017, 117 (22), 13461-13501.

25. Rood, M. T. M.; Spa, S. J.; Welling, M. M.; ten Hove, J. B.; van Willigen, D. M.; Buckle, T.; Velders, A. H.; van Leeuwen, F. W. B. Obtaining control of cell surface functionalizations via Pre-targeting and Supramolecular host guest interactions. Scientific Reports 2017, 7, 39908.

26. Willems, S. B. J.; Bunschoten, A.; Wagterveld, R. M.; van Leeuwen, F. W. B.; Velders, A. H. On-Flow Immobilization of Polystyrene Microspheres on $\beta$-Cyclodextrin-Patterned Silica Surfaces through Supramolecular Host-Guest Interactions. ACS Applied Materials \& Interfaces 2019, 11 (39), 36221-36231.

27. Schindelin, J.; Arganda-Carreras, I.; Frise, E.; Kaynig, V.; Longair, M.; Pietzsch, T.; Preibisch, S.; Rueden, C.; Saalfeld, S.; Schmid, B. Fiji: an open-source platform for biologicalimage analysis. Nature methods 2012, 9 (7), 676-682.

28. Ciaruttini, N. LiveGraphtoStack FIJI script. https://forum.image.sc/t/save-real-timeplot/7237 (accessed 13-06-2019).

29. Onclin, S.; Mulder, A.; Huskens, J.; Ravoo, B. J.; Reinhoudt, D. N. Molecular printboards: monolayers of beta-cyclodextrins on silicon oxide surfaces. Langmuir 2004, 20 (13), 5460-5466.

30. Picone, R.; Baum, B.; McKendry, R. Chapter 5 - Plasma Microcontact Patterning $(\mathrm{P} \mu \mathrm{CP})$ : A Technique for the Precise Control of Surface Patterning at Small-Scale. In Methods in Cell Biology, Piel, M.; Théry, M., Eds. Academic Press: 2014; Vol. 119, pp 73-90.

31. Chen, G.; Jiang, M. Cyclodextrin-based inclusion complexation bridging supramolecular chemistry and macromolecular self-assembly. Chemical Society Reviews 2011, 40 (5), 2254-2266.

32. Mulder, A.; Onclin, S.; Peter, M.; Hoogenboom, J. P.; Beijleveld, H.; ter Maat, J.; Garcia-Parajo, M. F.; Ravoo, B. J.; Huskens, J.; van Hulst, N. F.; Reinhoudt, D. N. Molecular printboards on silicon oxide: lithographic patterning of cyclodextrin monolayers with multivalent, fluorescent guest molecules. Small 2005, 1 (2), 242-253.

33. Mulder, A.; Auletta, T.; Sartori, A.; Del Ciotto, S.; Casnati, A.; Ungaro, R.; Huskens, J.; Reinhoudt, D. N. Divalent Binding of a Bis(adamantyl)-Functionalized Calix[4]arene to $\beta$ cyclodextrin-based Hosts: An Experimental and Theoretical Study on Multivalent Binding in Solution and at Self-Assembled Monolayers. Journal of the American Chemical Society 2004, $126(21), 6627-6636$.

34. Polini, A.; Prodanov, L.; Bhise, N. S.; Manoharan, V.; Dokmeci, M. R.; Khademhosseini, A. Organs-on-a-chip: a new tool for drug discovery. Expert Opinion on Drug Discovery 2014, 9 (4), 335-352. 
35. Dittrich, P. S.; Manz, A. Lab-on-a-chip: microfluidics in drug discovery. Nature Reviews Drug Discovery 2006, 5 (3), 210-218. 


\subsection{Supplementary Figures}

a
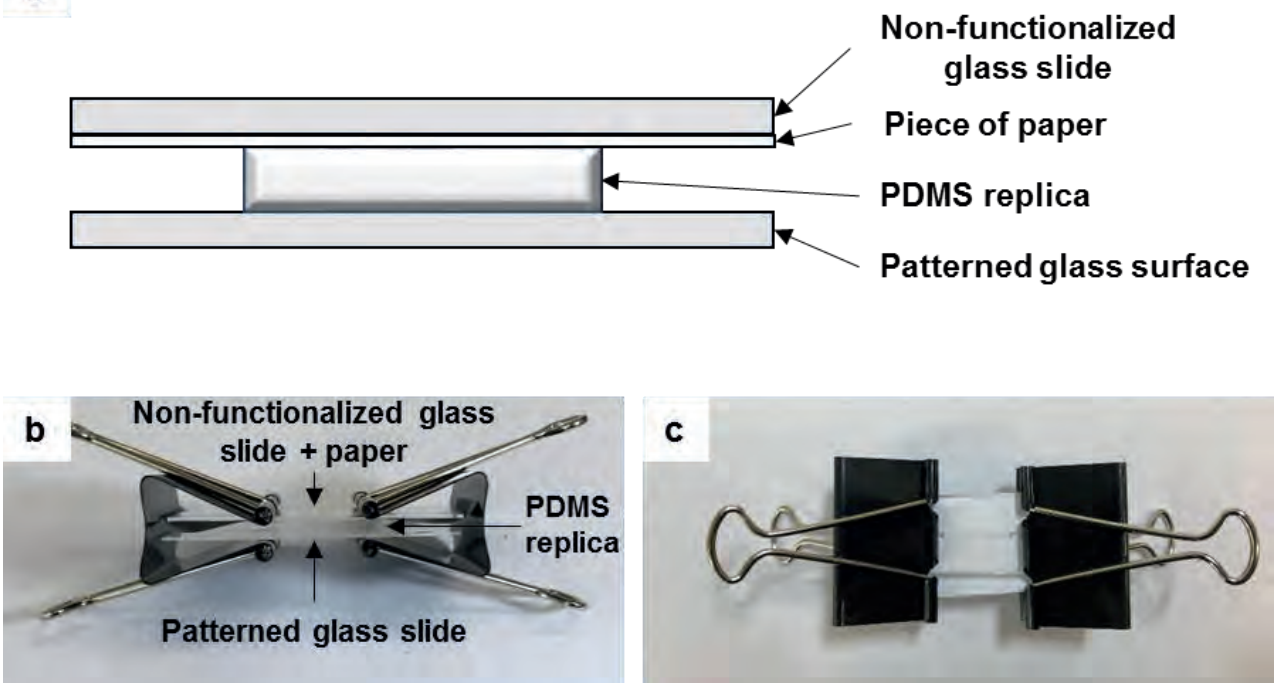

Fig. S1: Scheme showing setup of microfluidic with empty glass slide and piece of paper before attaching clamps (a). Photographs of microfluidic device being held in place by clamps before curing in the oven (b). Microfluidic device with clamp turned upside down to show functionalized glass surface and channel (c). 


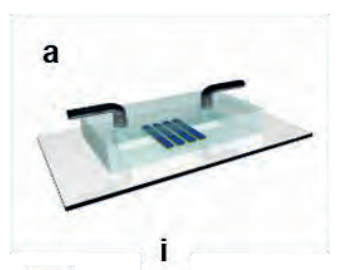

b
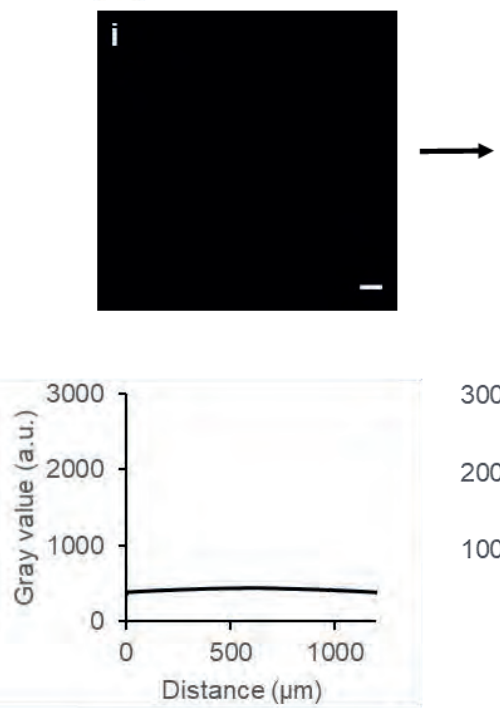

î
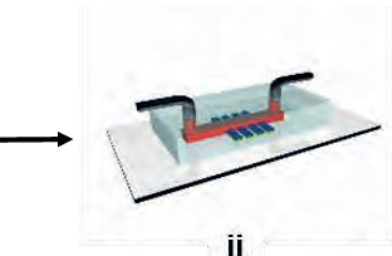

ii

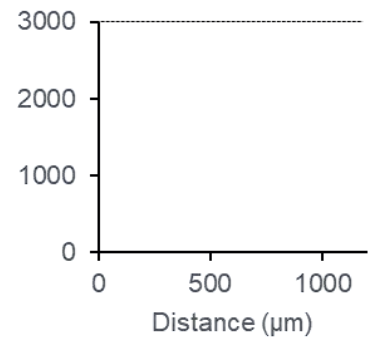

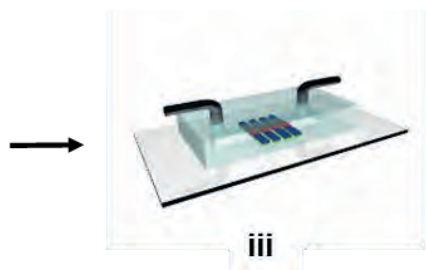

iii
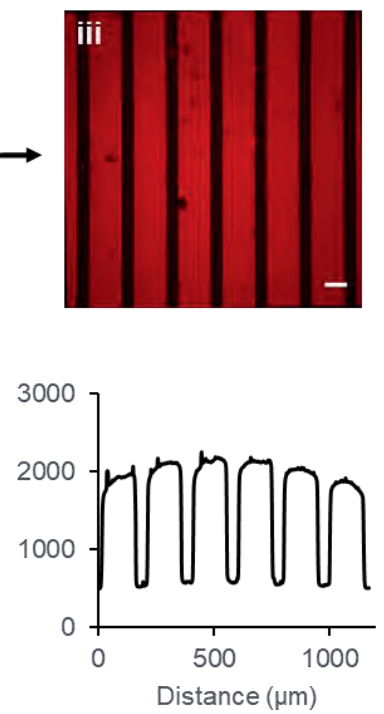

Fig. S2: a) Scheme depicting events for $\mathrm{Cy} 5-\mathrm{Ad}_{2}$ immobilization within microfluidic channels: (i) $\beta-\mathrm{CD}$ patterned microfluidic device before addition of $\mathrm{Cy} 5-\mathrm{Ad}_{2}$, (ii) $\mathrm{Cy} 5-\mathrm{Ad}_{2}$ addition over surface in device, and (iii) specifically immobilized Cy5- $\mathrm{Ad}_{2}$ on $\beta$-CD patterns. b) Microscopy images and constituent profile plots of (i) $\beta$ CD patterned microfluidic device before addition of $\mathrm{Cy} 5-\mathrm{Ad}_{2}$, (ii) during addition of $\mathrm{Cy} 5-\mathrm{Ad}_{2}$ (gray values on profile plot is above 3000 a.u. due to saturation) and (iii) after washing the surface with PBS and DI water. Scale bars are $100 \mu \mathrm{m}$. 


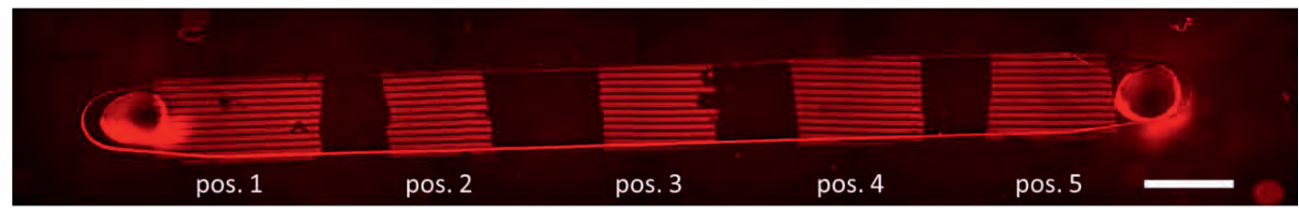

b
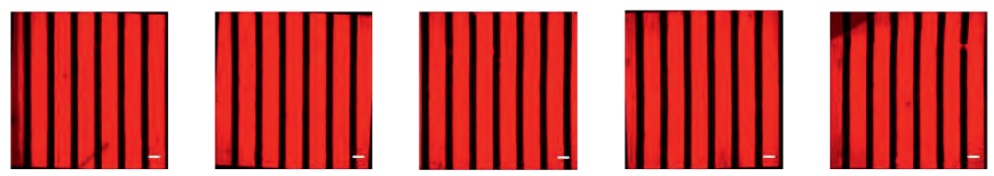

C

Signal to noise ratio

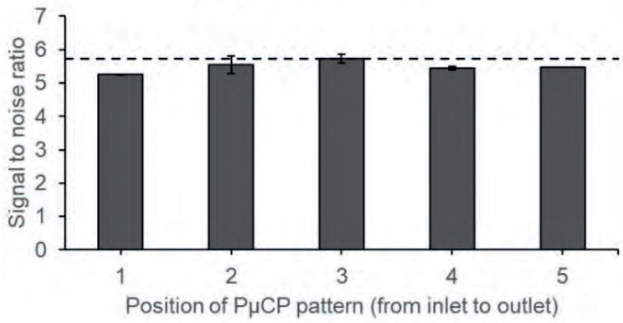

Fig. S3: Control experiment to test the effect of using multiple strips of a PDMS stamp for P $\mu \mathrm{CP}$ at different locations on one $\beta$-CD functionalized surface. a) Overview of the single channel after addition of $0.23 \mu \mathrm{M} \mathrm{Cy5}-\mathrm{Ad}_{2}$. Different positions of $\beta-C D$ patterns in the channels are marked. Image was captured with $5 x$ objective using $6 \times 1$ tilescan settings and scale bar is $2 \mathrm{~mm}$. b) $10 \mathrm{x}$ objective images of the separate positions after $90^{\circ}$ rotation which were used for carrying out profile plot analysis and determine signal to noise ratio of fluorescent patterns. c) Graph showing the average signal to noise ratio of patterns in different positions. 


\section{Script S1: FIJI Script for creating live profile plot videos (LiveGraphtoStack)}

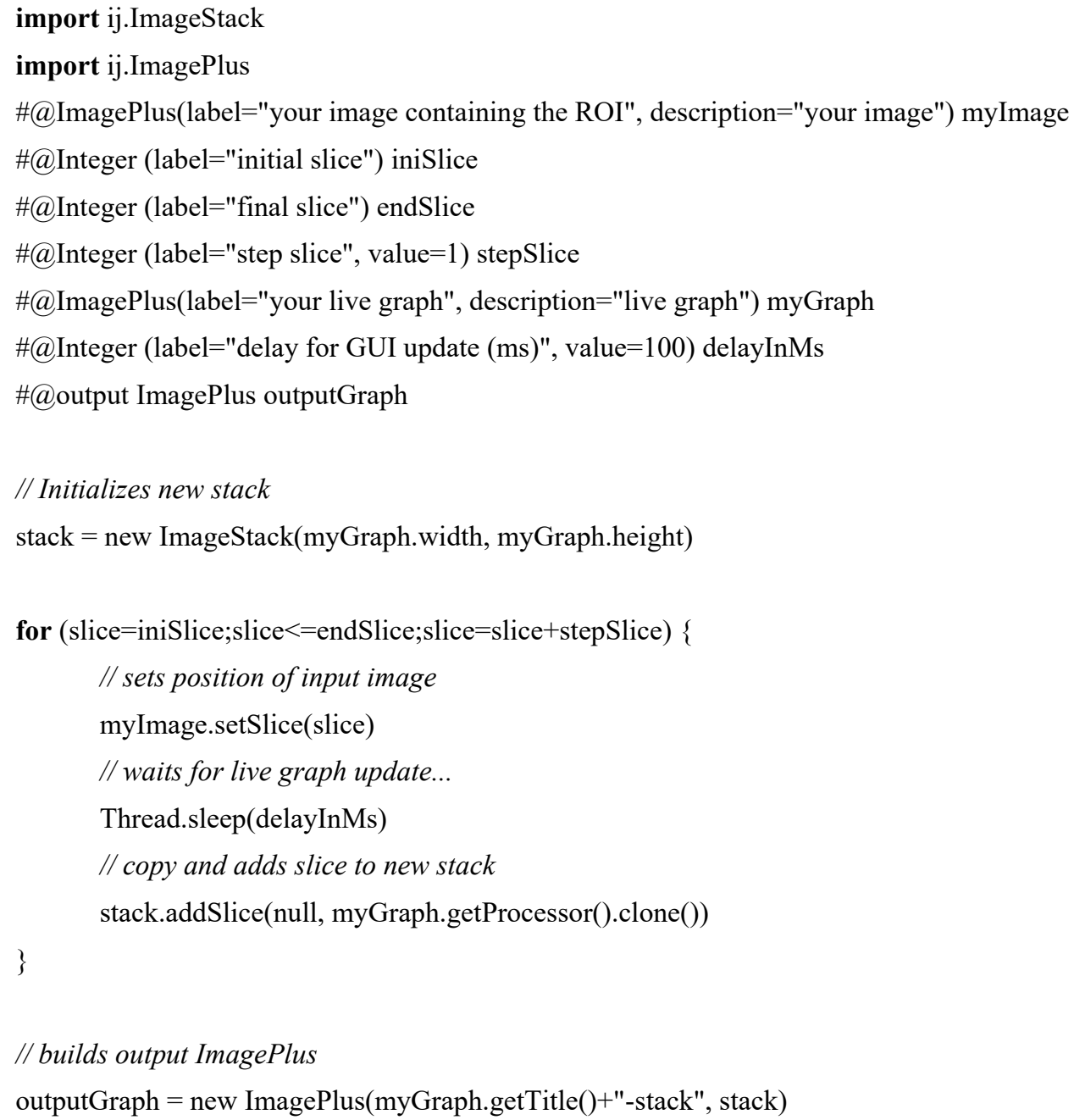




\section{Chapter 4}

\section{On-Flow Immobilization of Polystyrene Microspheres on $\beta$ - Cyclodextrin Patterned Silica Surfaces through Supramolecular Host-Guest Interactions}

Species specific isolation of micro-sized entities such as microplastics and resistant bacteria from waste streams is becoming a growing environmental challenge. By studying the on-flow immobilization of micron sized polystyrene particles onto functionalized silica surfaces, we ascertain if supramolecular host-guest chemistry in aqueous solutions can provide an alternative technology for water purification. Polystyrene particles were modified with different degrees of adamantane (guest) and silica surfaces were patterned with $\beta$-cyclodextrin ( $\beta$-CD, host) through microcontact printing $(\mu \mathrm{CP})$. The latter was exposed to solutions of these particles flowing at different speeds, allowing to study the effect of flow rate and multivalency on particle binding to the surface. The obtained binding profile were correlated with Comsol simulations. We also observed that particle binding is directly aligned with the particle's ability to form hostguest interactions with the $\beta$-CD patterned surface, as particle binding to the functionalized glass surface increased with higher adamantane load on the polystyrene particle surface. Due to the non-covalent character of these interactions, immobilization is reversible and modified $\beta$-CD surfaces can be recycled, which provides a positive outlook for incorporation in water purification systems.

This chapter has been published as: Stan B.J. Willems, Anton Bunschoten, Martijn Wagterveld, Fijs. W.B. van Leeuwen, and Aldrik H. Velders, ACS Applied Materials and Interfaces, 2019, 11 (39), 36221-36231. DOI: https://doi.org/10.1021/acsami.9b11069 


\subsection{Introduction}

Studying the interactions of microparticles with chemically modified surfaces is an important step in the development of cell targeting platforms. ${ }^{1,2}$ Immobilization of cells, for instance bacteria, to monolayers on surfaces provides an interesting field of research, especially towards medical or water treatment applications. ${ }^{3-6}$ However, within complex biological systems, such as bacterial cells, it is difficult to investigate specific surface adhesion interactions of micro-sized entities to functionalized surfaces. Importantly, the feasibility of particle adhesion interactions with respect to the different length scales being studied, i.e. between microparticles and molecules, should be confirmed using a more controllable model system. Therefore, the use of model microparticles such as polystyrene particles, which have the same size as most bacteria, is a versatile and valuable tool for investigating adhesion onto chemically functionalized surfaces.

In nature, the binding mechanisms of micro-sized entities such as cells are governed by numerous amounts of interactions. Among these interactions, hydrophobicity plays a significant role in the attachment or detachment of cells to surfaces. ${ }^{7}$ Therefore, Whitesides et $a l$. have carried out protein and cell adhesion studies by using monolayers of alkanethiolates or alkylsilanes on gold or silicon surfaces in order to mimic cell binding mechanisms within nature. ${ }^{8-11}$ Patterning of the surfaces through a soft lithographic technique, such as microcontact printing $(\mu \mathrm{CP}),{ }^{12,13}$ allowed for spatial control of the cell adhesion, which improved confirmation of binding during analysis. In order to acquire more specificity within cell adhesion studies, different targeting agents for bacteria were investigated by Sankaran et al., whom adhered bacteria to surfaces via an azobenzene-mannose linker and a transmembrane protein containing tryptophan and phenylalanine on cucurbit[8]uril layers. ${ }^{14-16}$ Moreover, Di Iorio et al. studied the binding kinetics of virus mimics to sialic acid residues via weak multivalent interactions in order to predict the number of interactions involved in binding. ${ }^{17}$ Based on these advances, the implementation and investigation of supramolecular, multivalent interactions for high affinity, noncovalent, and reversible binding is an important aspect for understanding cell immobilization. We here exploit this type of binding as an outlook toward water treatment applications, as it is desirable to capture cells rapidly and subsequently release immobilized cells for reusability of surfaces. 
a)

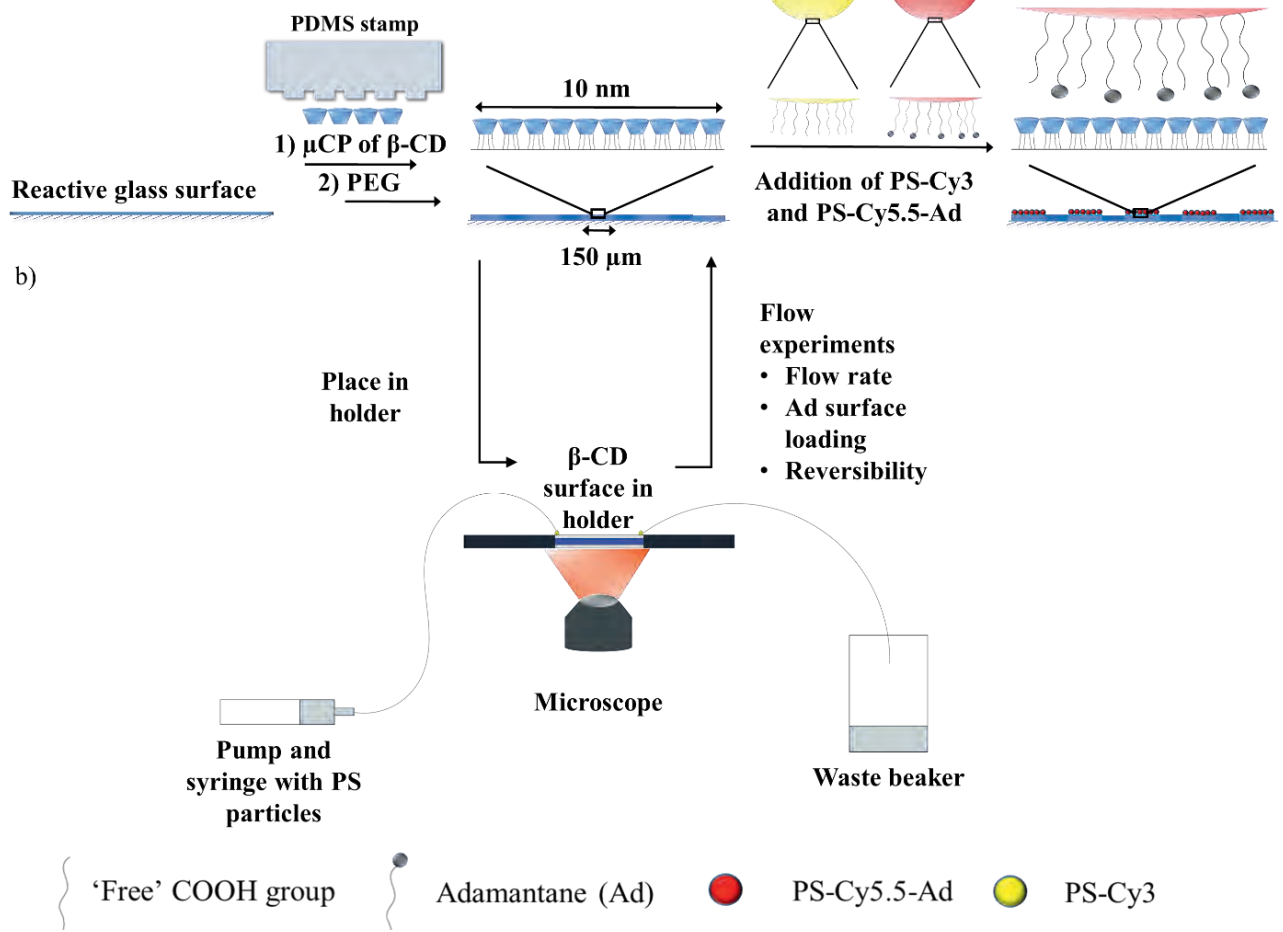

Fig. 4.1: a) Scheme showing $\mu \mathrm{CP}$ of $\beta-\mathrm{CD}$ on glass surface and backfilling with $\mathrm{PEG}$ and principle of binding: combined addition of non-functionalized polystyrene particles with $\mathrm{Cy} 3$ fluorescent label (PS-Cy3, negative control) and adamantane functionalized polystyrene particles with Cy5.5 fluorescent label (PS-Cy5.5-Ad) to patterned $\beta$-cyclodextrin surfaces. b) Flow cell holder setup on fluorescence microscope containing functionalized glass surface to perform the different types of flow experiments.

For fabrication of reusable surfaces we selected the noncovalent binding of adamantane to $\beta$-cyclodextrin. Cyclodextrins are cyclic carbohydrate molecules consisting of $1,4-\alpha-\mathrm{D}-$ glucopyranosides bound in a cone shape ${ }^{18}$ and are used primarily in food, pharmaceutical, and drug delivery applications. ${ }^{19,20}$ Hydrophobic molecules can form reversible inclusion complexes through hydrophobic and van der Waals interactions (host-guest chemistry) with the cyclodextrin cavity. Tethering of $\beta$-cyclodextrin $(\beta-C D)$ on silicon dioxide surfaces,${ }^{21}$ known as the molecular printboard, ${ }^{22}$ has been carried out by Reinhoudt and co-workers. This led to binding control and characterizing binding strength of functionalized dendrimers, nanoparticles and fluorescent guest molecules with cyclodextrin inside of its hydrophobic cavity. In later work by Gonzales-Campo et al., patterns of $\beta$-CD were created on glass surfaces through 
reactive $\mu \mathrm{CP}$, showcasing the possibility of fluorescent guest adsorption from solution to $\beta$-CD patterns or orthogonal supramolecular printing of fluorescent guests. ${ }^{23}$ The fluorescent guest molecules in these cases contain two adamantane (Ad) moieties, which have a high affinity for the $\beta$-CD cavity in water. ${ }^{24}$ Furthermore, the reversibility and re-usability of this system has been tested by forming multivalent networks of $\beta-\mathrm{CD}$ modified gold nanoparticles with diferrocene modified peptides, which functioned as an ultrasensitive enzyme sensor. ${ }^{25}$ In order to test the immobilization via host-guest interactions with larger constructs than molecules, silica nanoparticles and quantum dots have also been immobilized on $\beta-C D$ surfaces using an intermediary Ad functionalized dendrimer as 'glue'. ${ }^{26,27}$ The high binding affinity of multivalent host-guest interactions has also been used for the adhesion of macroscopic acrylamide gels. ${ }^{28}$ Based on these examples, the versatility of host-guest interactions mediated by $\beta$-CD provides a reliable tool for scaling up from nanoparticles to the specific and reversible immobilization of microparticles.

To study the supramolecular adhesion of microparticles, we functionalized polystyrene particles with different amounts of adamantane on their surface, patterned glass surfaces with $\beta-C D$ and subsequently applied a flow of functionalized particles over the modified glass surface (Fig. 4.1a). Polyethylene glycol (PEG) backfilling is included as an anti-fouling layer between $\beta$-CD patterns. The functionalized glass slides were placed in a holder which was connected to a syringe pump for controlling the flow rate of polystyrene particle solutions over the glass slides. Host-guest interactions between $\beta$-cyclodextrin and adamantane allow for the capture of the functionalized polystyrene particles on the glass surface, which can then be analyzed via fluorescence microscopy. Fig. 4.1b) shows how the flow cell holder setup is directly incorporated on the microscope to carry out various flow experiments for characterizing binding of PS particles to the $\beta$-CD functionalized glass slide. Results regarding the feasibility of this approach can also be used as a proof-of-concept for the application in immobilizing similar sized cells such as bacteria. 


\subsection{Experimental Section}

\section{Synthesis and functionalization of fluorescent PS particles}

$10 \mathrm{wt} \%, \sim 1 \mu \mathrm{m}$ polystyrene (PS) particles were synthesized following the protocol from Appel et al. using reaction mixture 'pSIA25'. ${ }^{29}$ Shortly, $125 \mathrm{~g}$ of DI water, $25 \mathrm{~g}$ of styrene, 0.5 $\mathrm{g}$ of itaconic acid and $0.01 \mathrm{~g}$ of fluorescent dye Cyanine 3 (Cy3) or Cyanine 5.5 (Cy5.5) (used for staining the core of resulting PS particles, structures shown in Fig. S1) were charged in a one neck round-bottom flask. The flask was sealed with a rubber septum and the reaction mixture was flushed with nitrogen for 15 minutes, followed by heating at $85{ }^{\circ} \mathrm{C}$ and stirring at $500 \mathrm{rpm}$ for $15 \mathrm{~min}$. A $0.1 \mathrm{M}$ solution of 4,4'-azobis(4-cyanovaleric acid) (ACVA) was prepared in $0.2 \mathrm{M} \mathrm{NaOH}$ and $8.92 \mathrm{~mL}(0.25 \mathrm{~g} \mathrm{ACVA})$ of this solution was injected to the reaction mixture to initiate colloid synthesis. The reaction was allowed to proceed for at least 12 hours at $85{ }^{\circ} \mathrm{C}$ and stirring at $500 \mathrm{rpm}$. The following day the reaction mixture was filtered, spun down through centrifugation at $3260 \mathrm{~g}$ for $30 \mathrm{~min}$, then washed three times with DI water through centrifugation at $3260 \mathrm{~g}$ for $30 \mathrm{~min}$ per washing step. The concentration in wt $\%$ was determined through freeze drying a known volume of PS particles then measuring the mass of the lyophilized particles. $5 \mathrm{~mL}$ of $0.5 \%$ wt of PS-Cy5.5 particles in $10 \mathrm{mM} 2-(N-$ morpholino)ethanesulfonic acid (MES) buffer, pH 5.0, were modified with $0.53 \mu$ mol, 1.33 $\mu \mathrm{mol}$ and $2.67 \mu \mathrm{mol}$ of 1-adamantylamine hydrochloride using $0.32 \mathrm{mmol}$ 1-ethyl-3-(-3dimethylaminopropyl) carbodiimide hydrochloride (EDC) according to the covalent coupling protocol from Bangs Laboratories, Inc. ${ }^{30}$ After overnight EDC coupling, the dispersion of PS particles was spun down in a centrifuge at $3260 \mathrm{~g}$ (30 min) and subsequently washed 3 times with DI water also by centrifugation at $3260 \mathrm{~g}$ (30 min per washing step) in order to remove non-reacted 1-adamantylamine.

\section{Dynamic light scattering (DLS) measurements}

Dispersions of PS particle samples were diluted to the appropriate concentration with DI water and then analysed on a Malvern Zetasizer Nano S using the size intensity values. The samples were measured 3 times with 10 runs per measurement at $25^{\circ} \mathrm{C}$. The size of all particles was determined to be ca $1 \mu \mathrm{m}$ (Fig. S2). 


\section{Conductometric titration}

As synthesized fluorescent PS-Cy5.5 particles were diluted 40x in $20 \mathrm{~mL}$ DI water. The $\mathrm{pH}$ was adjusted to $\mathrm{pH} 10$ by using $10 \mathrm{mM} \mathrm{NaOH}$ to deprotonate all the carboxyl groups on the PS particle surface. The conductivity of the dispersion of PS particles was then measured upon addition of $2 \mathrm{mM} \mathrm{HCl}$ in $40 \mu \mathrm{L}$ increments until ca $\mathrm{pH} 4.5$ was reached. The data was plotted as volume $2 \mathrm{mM} \mathrm{HCl}$ added vs conductivity of the PS solution. From this graph the total amount of $\mathrm{CO}_{2} \mathrm{H}$ groups on the PS particle surface could be determined. ${ }^{31}$

\section{${ }^{1}$ H NMR spectroscopy}

The supernatant of PS particles after EDC coupling with 1-adamantylamine hydrochloride was collected and concentrated using rotary evaporation. The resulting product was re-suspended in $1 \mathrm{~mL}$ deuterated water $\left(\mathrm{D}_{2} \mathrm{O}\right)$ with $1 \mathrm{mM}$ trimethylsilylpropionic acid (TMSP) as internal standard. A Bruker $500 \mathrm{MHz}$ NMR system was used for acquiring the ${ }^{1} \mathrm{H}$ NMR spectra. The characteristic adamantane peaks at $1.6 \mathrm{ppm}$ in supernatant samples were integrated to the TMSP peak at $0 \mathrm{ppm}$ to calculate the concentration of unreacted adamantane left in each sample. The adamantane left within the dispersion of PS particles could then be determined by the difference between the adamantane added to the reaction mixture compared to the unreacted adamantane left in the supernatant.

\section{Glass surface functionalization}

Isothiocyanate monolayers on glass were prepared as described by Onclin et al. ${ }^{21}$ Glass slides were cleaned and oxidised with piranha solution $\left(\mathrm{H}_{2} \mathrm{SO}_{4}(95-98 \%) / \mathrm{H}_{2} \mathrm{O}_{2}(35 \%), 3: 1 \mathrm{v} / \mathrm{v}\right.$; Warning! Piranha solutions must be handled with caution as they may unexpectedly detonate) for 45 minutes, rinsed with large amounts of deionized (DI) water, and dried under $\mathrm{N}_{2}$. The glass slides were placed in a high vacuum, pre-heated desiccator together with a glass vial containing $1 \mathrm{~mL}$ of 3-aminopropyltriethoxysilane (APTES, 99\%) and placed in an oven at 70 ${ }^{\circ} \mathrm{C}$ overnight. Following amine monolayer formation, the glass slides were removed from the desiccator and rinsed with toluene and dichloromethane. The glass slides were then cured for at least 1 hour in the oven at $70^{\circ} \mathrm{C}$. Next, the glass slides were immersed in $0.1 \mathrm{M}$ 1,4-phenylene diisothiocyanate in anhydrous toluene for 2 hours under argon atmosphere to yield 
isothiocyanate bearing layers. Following the immersion, the surfaces were rinsed with toluene and dichloromethane and used immediately for microcontact printing. Stamps were made by using a technique from Whitesides. ${ }^{10}$ Shortly, stamps were prepared by casting a 10:1 (w/w) mixture of poly(dimethylsiloxane) (PDMS) and curing agent (Sylgard 184, Dow Corning) onto a silicon master to yield PDMS stamps with $150 \mu \mathrm{m}$ broad line patterns and $50 \mu \mathrm{m}$ broad spacing between the lines. After overnight curing at $70{ }^{\circ} \mathrm{C}$, the PDMS stamps were cut out, oxidised by high energy air plasma for 1 minute and incubated in $0.72 \mathrm{mM}$ heptakis-(6-amino6-deoxy)- $\beta$-cyclodextrin heptahydrochloride aqueous solution, $\mathrm{pH} 8.0$, for 2 hours. Before printing, excess solution was dried off from the stamp surfaces using a stream of nitrogen. The PDMS stamps were then brought into conformal contact with the freshly prepared isothiocyanate functionalized glass surfaces for 30 minutes. After carefully removing the stamps, the printed glass substrates were rinsed thoroughly with DI water. The glass substrates were then incubated overnight in $1 \mathrm{mM}$ methoxy polyethylene glycol amine (PEG-NH $2, \mathrm{M}_{\mathrm{w}}$ $2000 \mathrm{Da}$, Iris Biotech $\mathrm{GmbH}$ ) aqueous backfilling solution, $\mathrm{pH} 8.0$ to react with the unreacted isothiocyanates. Before use, the glass substrates were rinsed with DI water. When not used within a day, substrates were stored in backfilling solution at $4{ }^{\circ} \mathrm{C}$.

\section{Validation of $\beta$-cyclodextrin patterns}

The $\beta$-cyclodextrin printed glass substrates were incubated for 15 minutes on a droplet of $0.28 \mu \mathrm{M}$ Cyanine 5 dye, functionalized with two adamantyl moieties (Cy5-Ad $2,{ }^{32}$ structure shown in Fig. S1), solution in PBS. After removal of the glass substrates from the solution, they were rinsed with DI water and dried with nitrogen. The substrates were then imaged by fluorescence microscopy.

\section{Flow experiments}

An equimolar mixture of PS-Cy3 and PS-Cy5.5-Ad at $\mathrm{pH} \sim 10$ was flowed through a resealable flow cell holder (fluidic connect PRO Resealable, Micronit; for dimensions see Fig. $\mathrm{S} 3 \mathrm{a}+\mathrm{b}$ ) containing a $\beta-\mathrm{CD}$ printed glass surface (4515 extended resealable flow cells, Micronit) using a syringe pump. The $\mathrm{pH}$ of the PS particles was increased to $\mathrm{pH} 10$ using $0.1 \mathrm{M} \mathrm{NaOH}$ to ensure that unreacted amine groups of heptakis-(6-amino-6-deoxy)- $\beta$-cyclodextrin on the glass surface were not protonated and therefore uncharged. Flow rates and pumping times of 
0.125 (40 hrs), 0.25 (20 hrs), 1.25 (4 hrs), 2.5 (2 hrs), 5 (1 hr), 10 (30 mins), 100 (3 mins) and 200 (1.5 mins) $\mu \mathrm{L} / \mathrm{min}$ were used to pump the particle solution over the glass surfaces. The pumping time, listed in brackets after the respective flow rate, was adjusted for different flow rates to keep the same total amount of PS particles flowing over the surface. The flow cell holder was placed directly on the stage of a Leica DMi8 epi-fluorescence microscope to allow imaging before, during and after flow. After flow incubation, the pump was switched off and the inlet tubing was removed, causing the solution to flow out of the resealable flow cell through capillary effect.

\section{Calculating particle density and binding specificity of particles}

The particle density on $\beta$-CD patterns was determined through analysis of microscope images after flow experiments. For acquiring an average particle density, all individual experiments were carried out 3 times and per glass slide the start, middle and the end of the flow path were analysed using ImageJ software ( 9 images in total). The pixels related to a nonaggregated particle were initially segmented out of the image using the 'Analyse particles' function. Subsequently, the average pixel size for one particle was determined. With the 'Measure' function, the pixels were counted over the desired line patterns (on $\beta$-CD patterns or PEG backfilling), which was then converted into amount of particles based on the determined average pixel size for one particle. The particle density over $100 \mu \mathrm{m}^{2}$ was subsequently calculated based on the total surface area of desired line patterns ( $\beta-C D$ or PEG patterns) per image. The binding specificity of particles for $\beta-C D$ printed patterns was then calculated as a percentage with the following formula:

$$
\eta=\frac{\sigma_{p}}{\sigma_{p}+\sigma_{n}} \times 100
$$

Here, $\eta(\%)$ is the binding specificity, $\sigma_{p}\left(\mu \mathrm{m}^{-2}\right)$ the particle density on the $\beta$-CD patterns and $\sigma_{n}\left(\mu \mathrm{m}^{-2}\right)$ the particle density on the PEG spacing. Throughout the results and discussion, the terms 'specificity' and 'selectivity' will be used frequently. Specificity is a comparison between binding of PS-Cy5.5-Ad to either $\beta$-CD or PEG while selectivity is a comparison between PS-Cy5.5-Ad or PS-Cy3 binding to $\beta$-CD. 


\section{Comsol simulations}

For simulating the flow experiments Comsol Multiphysics 5.4 with Particle Tracing Module was used. A 2D design of the microchannel was made $(20 \times 0.3 \mathrm{~mm}$, similar to the extended channel part of the Micronit resealable flow cell) with an inlet on the left, outlet on the right, and stick conditions for the top and bottom channel wall (Fig. S3c). For the simulations, particles of $1 \mu \mathrm{m}$ were chosen and the following conditions were applied: gravity, Brownian motion and creeping/laminar flow conditions. To simulate the different flow rates used, the average flow velocity and particle release times were varied between 0.0625 and $200 \mu \mathrm{L} / \mathrm{min}$ (shown in Table 1). The particle addition time entails the specific time points at which particles were infused into the designed channel and are given as start (in all cases at time $0 \mathrm{~s}$ ), the interval time between start and end times (i.e., if 200s, every 200s 40 particles are added) and the end time of the simulation for which no particles are added.

Table 4.1: Table showing conversion of flow rate to average flow velocity and the particle addition times for different flow velocities. 40 particles are added at time 0 s and at the given intervals with a total of 1480 particles for each simulation

Flow rate $\left(\mu \mathrm{L} \mathrm{\textrm {min } ^ { - 1 } )}\right.$

\begin{tabular}{ccc} 
& within flow channel $\left(\mathbf{m ~ s}^{-1}\right)$ & (start, interval, end) (s) \\
\hline 0.0625 & $2.14 \times 10^{-7}$ & $0,8000,288000$ \\
0.125 & $5.35 \times 10^{-7}$ & $0,4000,144000$ \\
0.25 & $1.07 \times 10^{-6}$ & $0,2000,72000$ \\
1.25 & $5.35 \times 10^{-6}$ & $0,400,14400$ \\
2.5 & $1.07 \times 10^{-5}$ & $0,200,7200$ \\
5 & $2.14 \times 10^{-5}$ & $0,100,3600$ \\
10 & $4.27 \times 10^{-5}$ & $0,50,1800$ \\
100 & $4.27 \times 10^{-4}$ & $0,5,180$ \\
200 & $8.54 \times 10^{-4}$ & $0,2.5,90$
\end{tabular}

Average flow velocity within flow channel $\left(\mathrm{m} \mathrm{s}^{-1}\right)$
Particle addition times (start, interval, end) (s) 


\subsection{Results and Discussion}

Patterned monolayers of $\beta$-cyclodextrin $(\beta-C D)$ were fabricated on glass surfaces and adamantane functionalized fluorescent polystyrene (PS) microparticles were synthesized with different degrees of Ad surface loading, ranging from 0.15-0.8 Ad mole fraction of available $\mathrm{CO}_{2} \mathrm{H}$ groups on the PS particle surface. Both the surface functionalization and synthesis of modified particles were characterized. Then, the immobilization of the particles on the glass surface in flow, via host-guest interactions, was validated through patterned binding of particles. For reference, a control PS particle without Ad functionality was always included to check the selectivity of the system. Various flow experiments were carried out to further investigate this model system, namely: flow rate variation, immobilization of PS particles with different Ad loading on PS surface, and re-usability of the glass slides after removal of bound PS particles.

\section{Fabrication of $\beta$-cyclodextrin $(\beta-C D)$ patterns through microcontact printing $(\mu \mathrm{CP})$}

Glass slides were first silanized with APTES and then functionalized with PDITC to create a surface reactive towards amines. The patterning of isothiocyanate glass slides with heptakis amino $\beta$-cyclodextrin $(\beta-\mathrm{CD})$ was achieved through microcontact printing $(\mu \mathrm{CP})^{13}$ and unreacted areas were filled with PEG-NH 2 (First step in Fig. 4.1b)). Patterning through $\mu \mathrm{CP}$ functions as an internal control to observe the specific assembly of molecules or particles on the $\beta$-CD surface specifically. According to literature, ${ }^{21,33}$ the presence of $\beta$-CD patterns on the surface can be validated through the addition of diadamantane functionalized fluorophores. We used a Cyanine 5 functionalized with two adamantane molecules $\left(\mathrm{Cy} 5-\mathrm{Ad}_{2}\right)$ for detecting $\beta-\mathrm{CD}$ patterns on the surface, which yielded a bright fluorescence signal over printed lines of $\beta$-CD (Fig. S4). Rinsing with DI water did not remove the bound Cy5-Ad 2 , which indicates the presence of a high affinity interaction between $\mathrm{Cy} 5-\mathrm{Ad}_{2}$ and $\beta-\mathrm{CD}$. 


\section{Polystyrene particles characterization and functionalization with adamantane amine}

PS particles covered with carboxyl groups were synthesized according to the protocol from Appel et al., ${ }^{29}$ with either Cy3 or Cy5.5 (Fig. S1) dyes used to stain the polystyrene cores. This allowed for facile analysis and discrimination using fluorescence imaging. After particle synthesis, the concentration of PS particles was determined: a known volume of the particles was lyophilized and the resulting particles were weighed to determine the mass concentration (Table S1). From this value, we calculated the as-synthesized particles to have a concentration of $1.82 \times 10^{11} \mathrm{PS}$ particles $/ \mathrm{mL}$ (Eq. S1). The amount of $\mathrm{CO}_{2} \mathrm{H}$ groups per PS particle was then determined at $2.4 \times 10^{7}$ through reverse conductometric titration (Fig. S5). ${ }^{31}$

PS-Cy5.5 particles were modified with $0.53,1.33$, and $2.67 \mu \mathrm{mol}$ of adamantane amine via an EDC coupling reaction. The Ad surface loading on PS particles was determined through ${ }^{1}$ H NMR spectroscopy of PS particle supernatant (Fig. S6 and S7) and PS particle samples were named after their degree of surface functionalization; PS-Cy5.5-0.15Ad, PS-Cy5.5-0.3Ad and PS-Cy5.5-0.8Ad (Table 4.1). However, for PS-Cy5.5-0.8Ad, a surprisingly high PS surface loading of 6 Ad molecules per $\mathrm{nm}^{2}$ was determined, which is unlikely because the effective size

Table 4.2: Determining degree of surface functionalization after NMR analysis of the supernatant of different PS-Cy5.5-Ad samples.

\begin{tabular}{|c|c|c|c|}
\hline PS-Cy5.5-Ad samples & & & \\
\hline $\begin{array}{c}\text { Ad- } \mathrm{NH}_{2} \text { added to PS } \\
(\mu \mathrm{mol})\end{array}$ & 0.53 & 1.33 & 2.67 \\
\hline $\begin{array}{l}\text { Ad- } \mathrm{NH}_{2} \text { amount in } \\
\text { supernatant }(\mu \mathrm{mol})\end{array}$ & 0.24 & 0.70 & 1.15 \\
\hline $\begin{array}{c}\text { Ad-NH } \\
(\mu \mathrm{mol})\end{array}$ & 0.29 & 0.63 & 1.52 \\
\hline $\begin{array}{c}\text { Amount of Ad molecules } \\
\text { on PS particle }\end{array}$ & $3.9 \times 10^{6}$ & $8.3 \times 10^{6}$ & $2 \times 10^{7}$ \\
\hline $\begin{array}{c}\text { Ad molecule(s) per } \mathrm{nm}^{2} \\
\text { of PS particle surface } \\
\text { Degree of surface }\end{array}$ & $\sim 1$ & $\sim 2$ & $\sim 6$ \\
\hline $\begin{array}{l}\text { functionalization/mole } \\
\text { fraction }\end{array}$ & 0.15 & 0.3 & 0.8 \\
\hline
\end{tabular}


of adamantane is ca $0.41 \mathrm{~nm}^{2}$ (based on reported adamantane radius of $0.36 \mathrm{~nm}$ ). ${ }^{34}$ This can be explained by the surface roughness of PS particles which increases the total surface area compared to the model of a smooth sphere used for carrying out all the calculations. ${ }^{35}$ The high values for adamantane surface loading can also be explained by non-specific uptake of adamantane amine in the dispersion of PS particles, which was determined by omitting EDC during coupling (Table S2). Nevertheless, the adamantane loading determined through NMR analysis shows significant differences between different concentrations of Ad used for modifying PS particles and should therefore be seen as a relative loading rather than an absolute value.

\section{Flow rate variation}

The immobilization of the micrometer sized particles was tested under different flow conditions ranging from 0.125 to $200 \mu \mathrm{L} / \mathrm{min}$. PS-Cy5.5-0.8Ad particles (red) were successfully captured on $\beta$-CD lines as observed in images i-iv of Fig. 4.2a), confirming that microparticle immobilization is feasible under flow conditions. Selectivity of adamantane binding was shown by mixing in Cyanine 3 stained polystyrene particles (PS-Cy3, yellow) without adamantane (Fig. 4.2a) v-viii). A reference surface was created by $\mu \mathrm{CP}$ glycine, instead of $\beta-C D$, confirmed that the immobilization of adamantane functionalized particles on $\beta-C D$ functionalized surfaces was the result of specific host-guest interactions (Fig. S8; $5 \mu \mathrm{L} / \mathrm{min}$ for 1 hour).

Rinsing of the functionalized glass surfaces with DI water did not remove specifically immobilized particles, indicating strong binding interactions. The patterned binding of the adamantane modified PS particles to the $\beta$-CD modified glass surface confirms that the binding affinity is high enough to overcome drag forces acting on particles (see Eq. S1 and Table S3); the drag force acting on a microparticle for all flow rates is approximately $10^{5}$ times lower than the binding strength of an individual Ad- $\beta$-CD host-guest complex $\left(10^{-11} \mathrm{~N}\right) .{ }^{36}$ However, this value for Ad- $\beta-C D$ binding strength is based on ideal conditions, not considering kinetic and concentration effects which are also important for binding affinity and particle immobilization. Therefore, it is still necessary to have multiple Ad- $\beta-C D$ bonds for keeping particles immobilized on surfaces during flow. 
a)
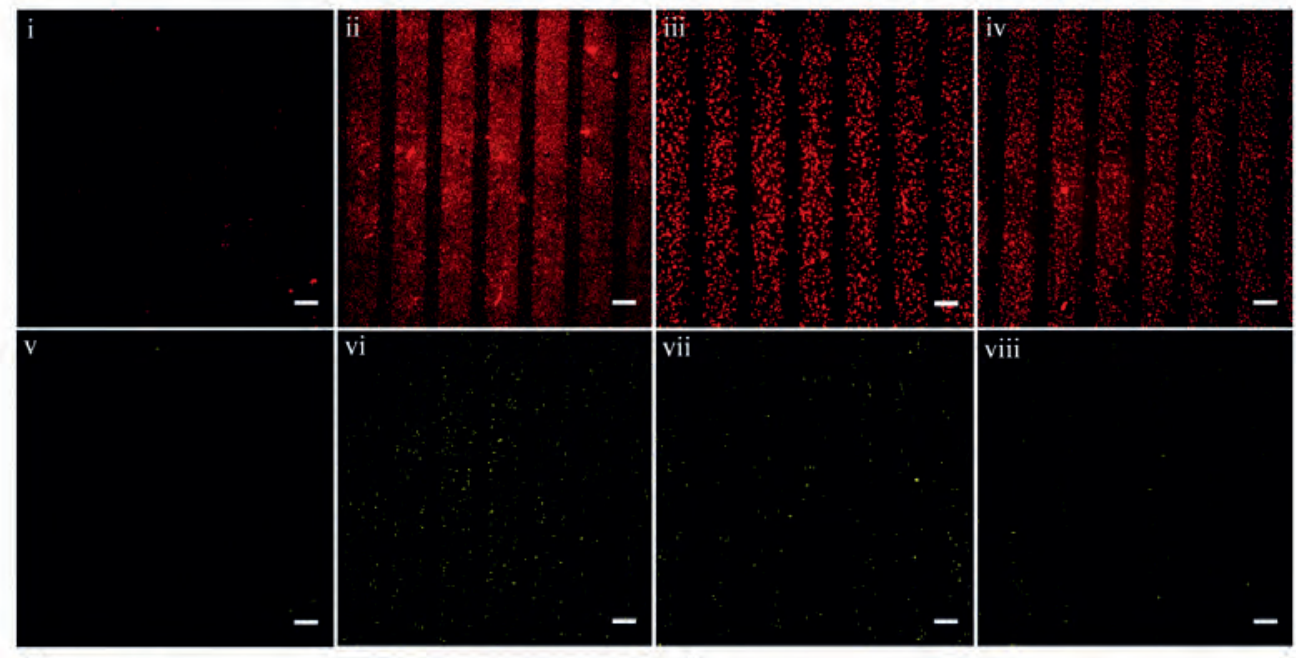

b)

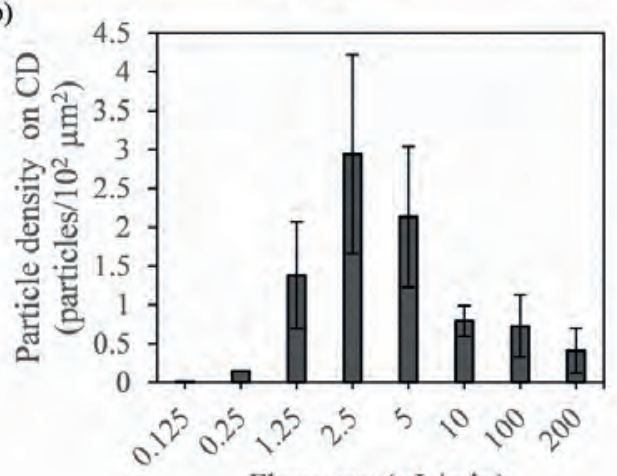

c)

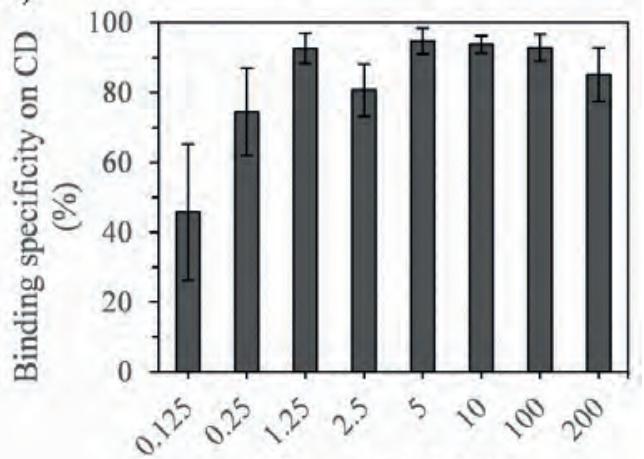

Flow rate $(\mu \mathrm{L} / \mathrm{min})$

Fig. 4.2: a) Fluorescence images of patterned $\beta$-CD glass surfaces after addition of an equimolar mix of fluorescent polystyrene particles PS-Cy5.5-Ad (i-iv) and PS-Cy3 (v-viii). The patterns are oriented perpendicular to the flow direction. From left to right a selection of flow rates are shown: 0.125 (i, v), 2.5 (ii, vi), 100 (iii,vii), and 200 (iv,viii) $\mu \mathrm{L} / \mathrm{min}$. All flow rate experiments are shown in Fig. S9. Top images (red) are captured with a Cy5 filter and bottom images (yellow) with a RHOD filter. Images of different filters and same flow rate are captured at the same location to compare the effect of aspecific interactions. Scale bars are $100 \mu \mathrm{m}$. b) Graph showing the PS-Cy5.5-Ad particle density on $\beta$-cyclodextrin patterns versus all the flow rates used. c) Graph showing the binding specificity for these respective patterns versus the flow rate.

The influence of the flow rate on particle capturing was studied by varying the flow rate from $0.125 \mu \mathrm{L} / \mathrm{min}$ to $200 \mu \mathrm{L} / \mathrm{min}$. Subsequently, the different flow rates were compared both on density of captured particles and on specificity (Fig. 4.2 and Fig. S9). The values for particle binding density and specificity of PS-Cy5.5-0.8Ad particles for the printed $\beta$-CD lines on the glass surface were calculated and plotted in Fig. 4.2b and c. Looking at the particle binding 
density graph in Fig. 4.2b and microscope images (Fig. 4.2a) i-iv), increasing the flow rate from $2.5 \mu \mathrm{L} / \mathrm{min}$ to $200 \mu \mathrm{L} / \mathrm{min}$ resulted in a decrease of immobilized particles from 3 to 0.5 PSCy5.5-0.8Ad particles $/ 10^{2} \mu \mathrm{m}$ on $\beta$-CD. This finding is in line with literature as particle deposition increases with lower flow rates. ${ }^{37}$

Another important factor for assessing the immobilization of particles to modified surfaces, is the particle binding specificity. The particle binding specificity, in this case, measures how well PS-Cy5.5-0.8Ad particles bind specifically to the patterns of $\beta$-CD compared to the aspecific binding to PEG backfilling and is plotted in Fig. 4.2c). From a flow rate of $2.5 \mu \mathrm{L} / \mathrm{min}$ and upwards, samples have a binding specificity of $\geq 80 \%$ for $\beta$-CD lines, with flow rates from $5 \mu \mathrm{L} / \mathrm{min}$ and higher having the highest specificities above $90 \%$. Using a flow rate of 0.125 and $0.25 \mu \mathrm{L} / \mathrm{min}$ resulted in a lower binding specificity most significantly compared to the other flow rates, which is mostly due to the lower particle binding density. The amount of particles captured on the functionalized surfaces at flow rates from $10 \mu \mathrm{L} / \mathrm{min}$ and higher is considerably lower than with 2.5 and $5 \mu \mathrm{L} / \mathrm{min}$ flow rates (Fig. 4.2b)). Thus, a flow rate of $5 \mu \mathrm{L} / \mathrm{min}$ for specific immobilization of these particles is most desirable considering the particle binding density and specificity.

Surprisingly, when we carried out particle capturing experiments at extremely low flow rates of 0.125 and $0.25 \mu \mathrm{L} / \mathrm{min}$, almost no particles were bound to the printed $\beta$-CD surface. Comsol particle flow simulations were carried out to investigate the change in particle binding to the $\beta$-CD modified surface with flow rates. These calculation were made using a $2 \mathrm{D}$ flow cell geometry of $20 \mathrm{~mm}$ in length and $0.3 \mathrm{~mm}$ in height that represented the conditions in the Micronit ${ }^{\circledR}$ flow channel, $1 \mu \mathrm{m}$ sized particles, similar flow rates, and stick conditions for both bottom and top channel walls (Fig. S3c). After carrying out simulations at different flow rates, particles were counted over the center of the bottom channel wall from 5-15 mm for each simulation, which is approximately where the glass surface in the practical experiments was patterned with $\beta$-CD through $\mu \mathrm{CP}$. Indeed, a similar trend in particle binding density to the practical flow experiments was noticed in the Comsol particle tracing data when we focused on this area (Fig. 4.3a-i), marked with a red line). In Fig. 4.3, it is also clear that the flow profile of trajectory graphs a)-d) (0.0625-1.25 $\mu \mathrm{L} / \mathrm{min}$ flow rate) is mostly based on diffusion and settling of particles, while in trajectory graphs e)-f) $(2.5-200 \mu \mathrm{L} / \mathrm{min}$ flow rate), a laminar flow profile is observed. In trajectory graphs a) and b) from Fig. 4.3, the particles stuck to the marked area on the bottom channel wall decreases dramatically because particles settle before they 
a)

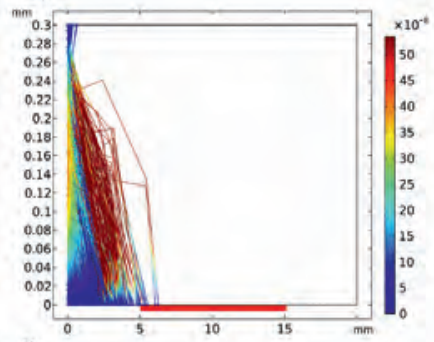

d)

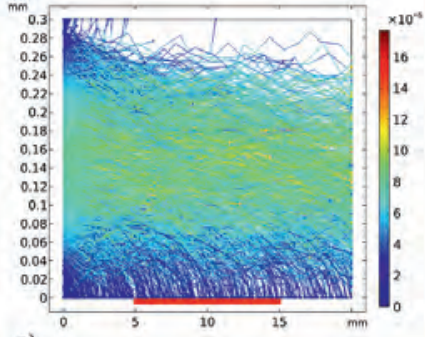

g)

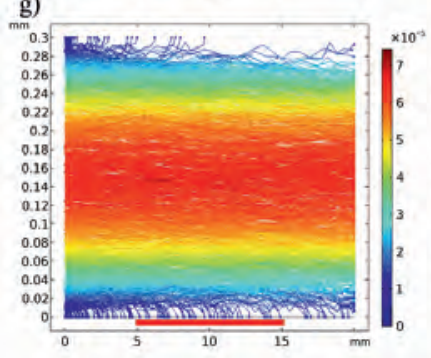

b)

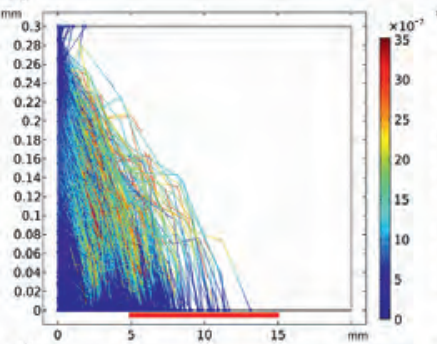

e)

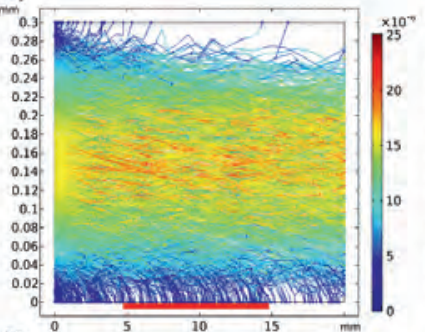

h)

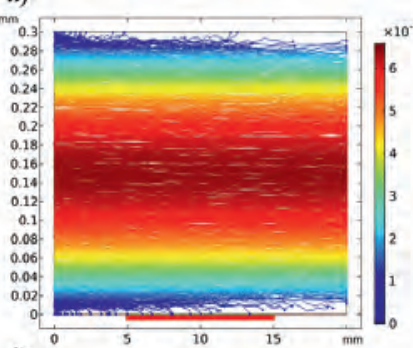

j)

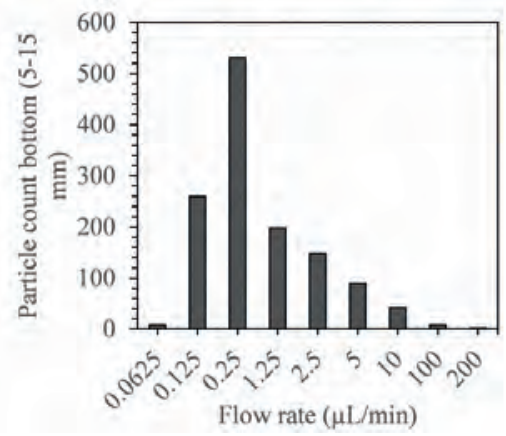

c)

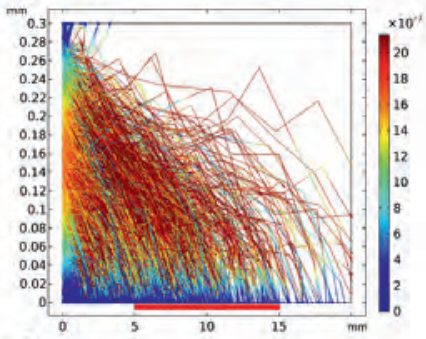

f)

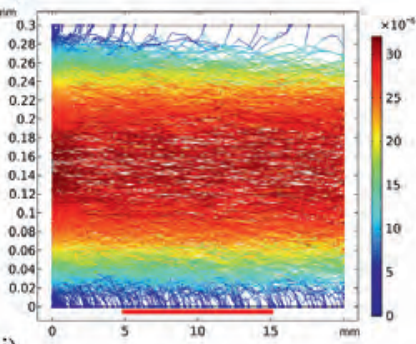

i)

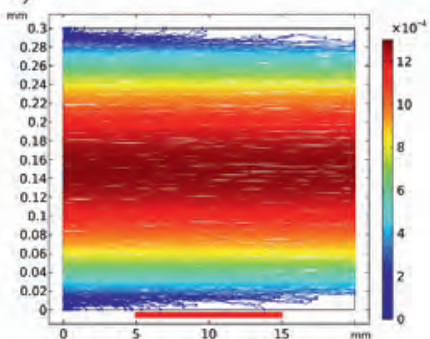

Fig. 4.3: Comsol particle tracing data of flow rates a) 0.0625 , b) 0.125 , c) 0.25 , d) 1.25 , e) 2.5 , f) 5 , g) 10 , h) 100 , and i) $200 \mu \mathrm{L} / \mathrm{min}$. The red line marks the area that was focused on for particle counting. The scale on the right side of each image for a-i) shows the particle velocity in $\mathrm{m} / \mathrm{s}$. Particle size is scaled up for viewing purposes. Both bottom and top channel walls have stick condition. k) Graph showing the amount of particles stuck on the bottom channel wall from Comsol simulation data vs flow rate used. The total amount of particles was 1480 in each simulation. 
reach the designated area. The trend seen in the graph in Fig. 4.3j) correlates well with experimental findings for the practical experiments in Fig. 4.2. Therefore, a decrease is observed in particle binding density due to the particles settling before reaching the functionalized area with $\beta-C D$ patterns in the middle of the channel. This was confirmed by carrying out a flow experiment at $0.125 \mu \mathrm{L} / \mathrm{min}$ with only PS-Cy3 particle on a PEG functionalized glass surfaces, which shows that most particles are situated on the inlet side of the flow channel after flow incubation (Fig. S10).

Adversely, when increasing the flow rate within a laminar flow regime (between flow rates of 2.5 and $200 \mu \mathrm{L} / \mathrm{min}$ ), the particle binding density also decreases within the practical experiments and simulation because the effect of gravity and diffusion is lower. The effect of diffusion can be explained using the Péclet $(\mathrm{Pe})$ number, which describes the effect of flow advection compared to particle diffusion. As mentioned before, we assume laminar flow within the microfluidic channel at flow rates $2.5-200 \mu \mathrm{L} / \mathrm{min}$ which means that the particle velocity decreases close to the channel walls (Eq. S2, Table S4). When the advection, i.e. flow rate, increases and Pe numbers increase far above 1, the particle diffusion can be seen as negligible. In Table S4, the Pe numbers were calculated for the different flow velocities of the particles 1 $\mu \mathrm{m}$ above the channel wall using Eq. S3 and $\mathbf{S 4}$. For flow rates of 100 and $200 \mu \mathrm{L} / \mathrm{min}$, the Pe numbers were considerably higher than 1 which explains the small difference in particle binding density. This is caused by a limitation in the amount of particles that can reach the bottom channel through diffusion or settling, while at flow rates from 2.5 to $10 \mu \mathrm{L} / \mathrm{min}$, particle diffusion to the channel wall is possible from a larger distances (Fig. S11 and S12). Therefore, there is a specific range of flow rates in which the increased effect of diffusion combined with advection allows for more adamantane functionalized polystyrene particles to come into contact with the area patterned with $\beta-\mathrm{CD}$ and thus be immobilized. 
a)
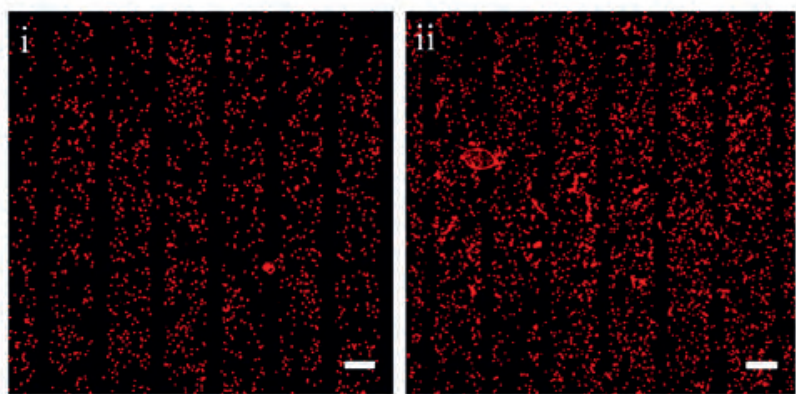

\section{Upside down flow}
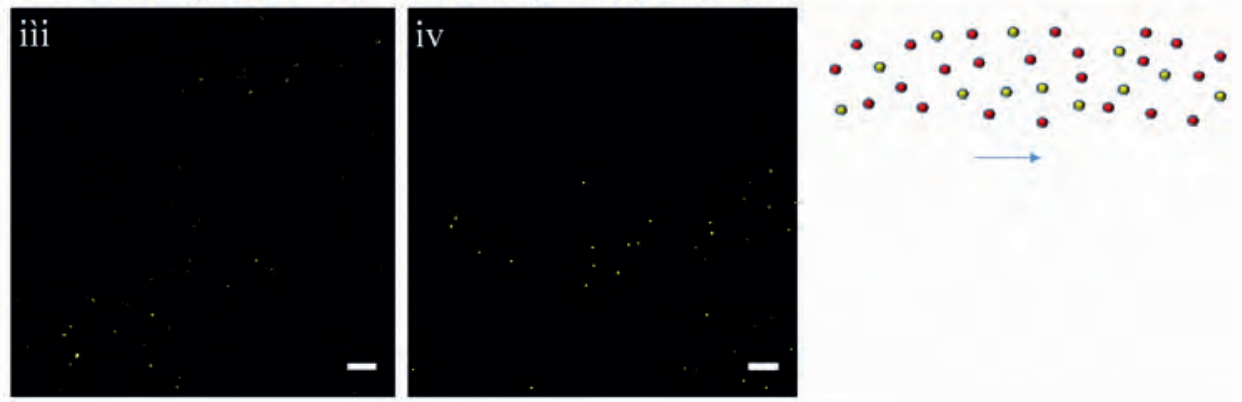

b)

c)
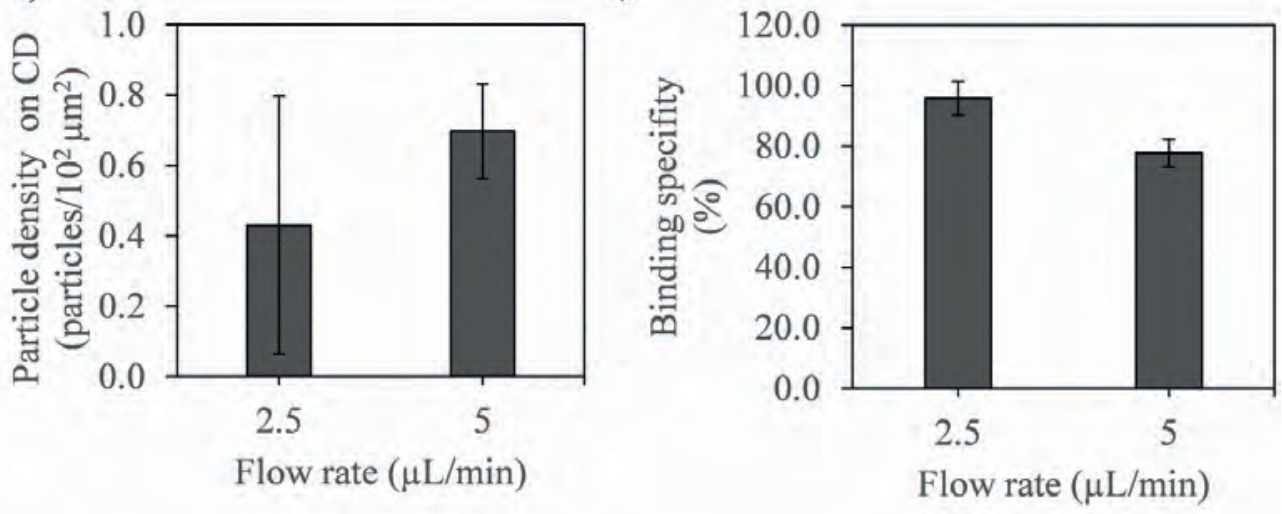

Fig. 4.4: a) Fluorescence microscope images of equimolar ratios of PS-Cy5.5-0.8Ad (i, ii) and PS-Cy3 particles (iii, iv) bound to $\beta$-CD patterned glass surfaces after upside down flow incubation at $2.5 \mu \mathrm{L} / \mathrm{min}$ (i, iii) and $5 \mu \mathrm{L} / \mathrm{min}$ (ii, iv). The patterns are oriented perpendicular to the flow direction. Top images are captured with Cy5 filter and bottom images with RHOD filter. Scale bars are $100 \mu \mathrm{m}$. b) Graph showing particle density vs flow rate for upside down flow on $\beta$-CD patterns. c) Graph showing binding specificity vs flow rate for upside down flow on $\beta$-CD patterns.

For non-functionalized PS-Cy3 particles, significantly lower binding, ranging from 0.008 to 0.04 particles per $100 \mu \mathrm{m}^{2}$ (for flow rates $2.5 \mu \mathrm{L} / \mathrm{min}$ to $200 \mu \mathrm{L} / \mathrm{min}$ ), was observed 
(Fig. S13). The amount of PS-Cy3 particles did however, increase when higher amounts PSCy5.5-Ad particles were immobilized on the surface, which could be caused by aggregation of PS-Cy3 with PS-Cy5.5-Ad particles. An experiment with only PS-Cy3 particles carried out as a control at $5 \mu \mathrm{L} / \mathrm{min}$ flow showed that almost no particles were bound to the glass surface (Fig. S14).

To study the effect of particle settling on PS-Cy5.5-0.8Ad particle binding to the functionalized surface, an upside down flow experiment at 2.5 and $5 \mu \mathrm{L} / \mathrm{min}$ was carried out by turning the flow cell holder upside down (Fig. 4.4). These flow rates were chosen because at 2.5 and $5 \mu \mathrm{L} / \mathrm{min}$ the transition to laminar flow occurs according to the particle trajectories in Fig. 4.3. As observed in the microscope images (Fig. 4.4a), PS particle binding to $\beta$-CD patterns is still possible in upside down flow situations and was selective for PS-Cy5.5-0.8Ad particles. Compared to the upright flow experiment in Fig. 4.2b), binding to the surface was $\sim 6$ times lower for $2.5 \mu \mathrm{L} / \mathrm{min}$ at 0.4 particles per $100 \mu \mathrm{m}^{2}$ and $\sim 3$ times lower for $5 \mu \mathrm{L} / \mathrm{min}$ at 0.8 particles per $100 \mu \mathrm{m}^{2}$ on $\beta$-CD patterns (Fig. 4.4b). Therefore, particle settling has a significant effect in improving the contact of the PS particles with the bottom channel wall. Nevertheless, PS-Cy5.5-0.8Ad particle binding to $\beta$-CD patterns is still feasible and specific through hostguest interactions without the aid of particle settling (Fig. 4.4c).

\section{Adamantane loading effect on PS particle binding}

The PS-Ad samples with different surface coverages of adamantane were flowed over the $\beta$-CD glass surfaces and were compared (Fig. 4.5). At a flow rate of $5 \mu \mathrm{L} / \mathrm{min}$, a clear increase in particle density is observed for PS particles with higher Ad surface coverage; 0.15 Ad loaded PS-Cy5.5 particles obtain a clear lower particle surface density $(0.4$ particles/100 $\mu \mathrm{m}^{2} \beta-\mathrm{CD}$ ) than 0.3 and 0.8 Ad loaded PS particles ( 1 and 2 particles $/ 100 \mu \mathrm{m}^{2}$, Fig. 4.5a and b). An increase in the negative control PS-Cy3 particle density on $\beta-\mathrm{CD}$ is also seen with higher Ad loading of PS-Cy5.5- Ad (Fig. S9). However, the PS-Cy3 particle density is still approximately 100 times lower than PS-Cy5.5-Ad density on cyclodextrin. PS-Cy5.5-Ad particle binding was in all cases very specific for $\beta$-CD patterns. 

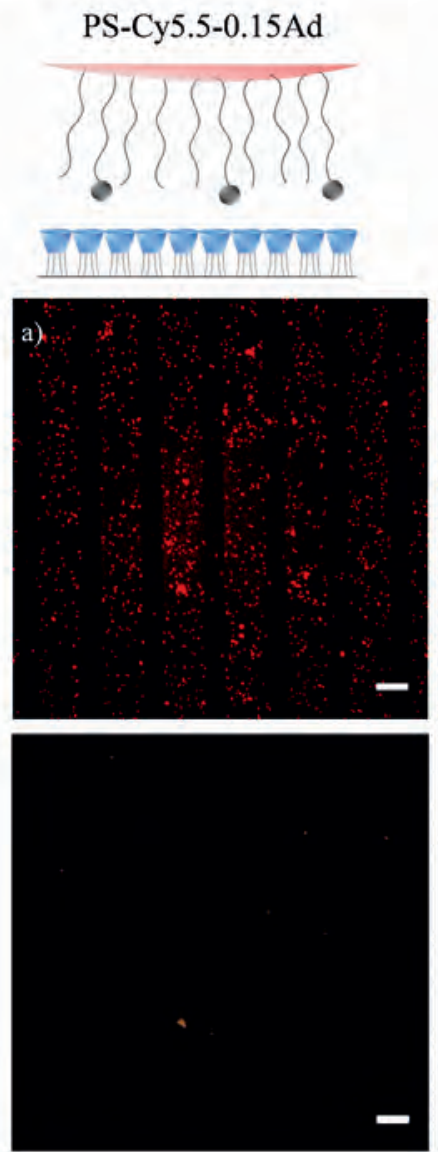

b)

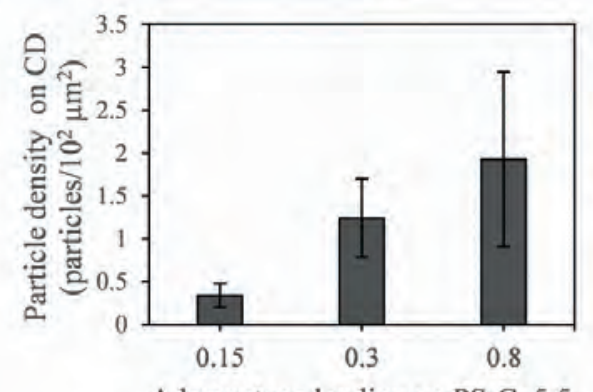

Adamantane loading on PS-Cy5.5
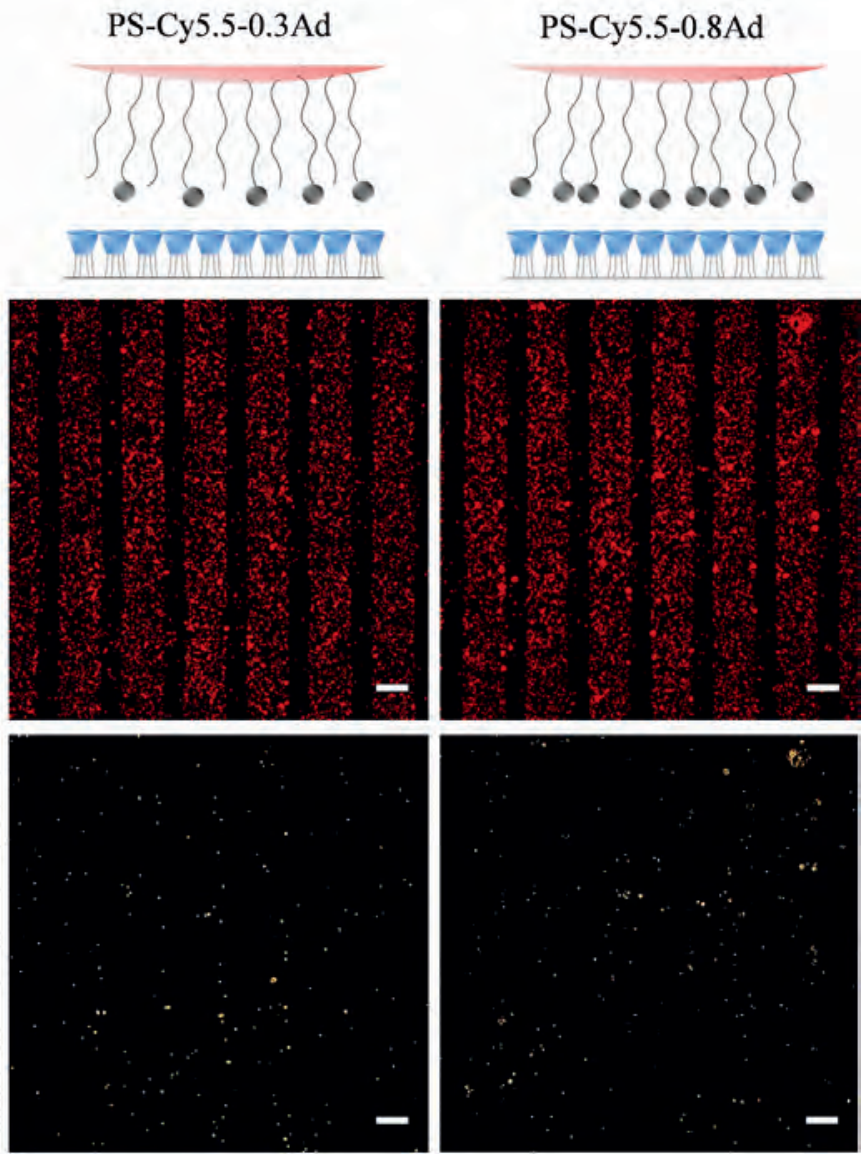

c)

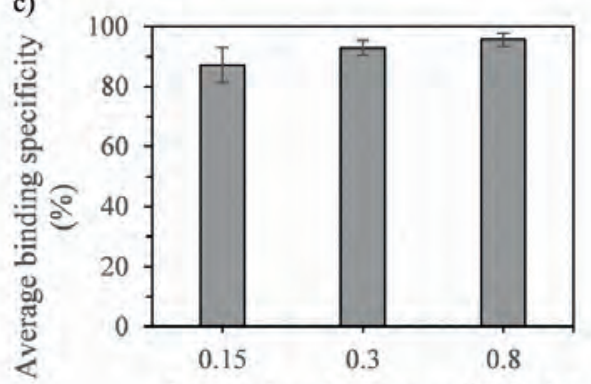

Adamantane loading on PS-Cy 5.5

Fig. 4.5: a) Fluorescence microscope images of PS particles adhered to $\beta$-CD lines with different Ad loading on PSCy5.5-Ad particles of $0.15,0.3$, and 0.8 at flow rate $5 \mu \mathrm{L} / \mathrm{min}$. The patterns are oriented perpendicular to the flow direction. Cy5 filter (red) shows PS-Cy5.5-Ad particles and RHOD filter (yellow) shows PS-Cy3 particles. Scale bar is $100 \mu \mathrm{m}$. b) Graph showing particle density on $\beta$-CD vs Ad loading on PS-Cy5.5 particle surface. c) Graph showing binding specificity for $\beta$-CD patterns vs Ad loading on PS-Cy5.5 particle surface. 
To shed some light on the amount of $\mathrm{Ad}$ and $\beta-\mathrm{CD}$ pairs that are interacting to immobilize one Ad-functionalized PS particle, the accessible contact area of PS and $\beta$-CD was calculated to be ca. $2000 \mathrm{~nm}^{2}$ when a PS particle touches the glass surface (based on an Ad$\mathrm{NH}_{2}$ linker length of $0.6 \mathrm{~nm}$ ). Assuming the Ad loading previously determined, the amount Ad moieties present on the PS particle surface within the $2000 \mathrm{~nm}^{2}$ contact area is approx. 2000 for PS-Cy5.5-0.15Ad, 4000 for PS-Cy5.5-0.3Ad, and 12000 for PS-Cy5.5-0.8Ad. When assuming a $\beta-C D$ surface density of $6 \times 10^{-11} \mathrm{~mol} / \mathrm{cm}^{2}$ on glass from literature, ${ }^{26} \mathrm{ca} .800 \beta-C D$ units are present in $2000 \mathrm{~nm}^{2}$ on glass. Interestingly, $\beta$-CD-Ad couples are therefore limited by the number of $\beta-C D$ molecules rather than Ad. However, as mentioned before in the characterization of the PS particles, the actual Ad loading on the PS surface is most likely lower than the amounts calculated via NMR analysis. More importantly, the surface roughness of PS particles and glass can also reduce the amount of accessible Ad and $\beta$-CD molecules for hostguest interactions. Furthermore, the binding affinity is also governed by concentration gradients within a solution and kinetic effects, which increases the complexity in determining the amount of interactions required for microparticle immobilization to a surface. Therefore, we argue that these experimental findings and analyses show that determining the amount of interactions required for microparticle immobilization is not straightforward and requires more research. Nevertheless, these results do show that changing the relative amount of targeting molecules on the microparticle surface, thereby tuning the multivalency, plays an important role for increasing microparticle immobilization on functionalized surfaces.

\section{Reversibility and re-usability of $\beta$-cyclodextrin glass surfaces}

The reversible nature of the particle immobilization mediated by host-guest interactions was tested by removal of particles and re-addition of 'new' particles (Fig. 4.6). The addition of the PS particle mix (PS-Cy5.5-0.8Ad and PS-Cy3) at $5 \mu \mathrm{L} / \mathrm{min}$ was carried out over the $\beta$-CD surfaces as before and the surface was analyzed on the microscope. In preliminary experiments, rinsing with $\mathrm{EtOH}$ was attempted to diminish the hydrophobic interaction between $\beta-\mathrm{CD}$ and adamantane. ${ }^{38}$ Also rinsing with a concentrated solution of $\beta-C D$ was performed to compete in the host-guest interaction. ${ }^{33}$ Unfortunately, both approaches did not result in significant release of the multivalently bound microparticles. Therefore, a more rigorous ultrasonic treatment in EtOH and subsequently water was applied. It is observed in Fig. 4.6a that complete removal of 
PS particles was achieved and that the glass surface is still functional for the subsequent particle immobilization experiment. Quantitative analysis for the two particle immobilization experiments shows that two times less PS-Cy5.5-Ad particles are immobilized the $2^{\text {nd }}$ time (Fig. 4.6b). This could be due to loss of $\beta-C D$ functionality through ultrasonic treatment or because some EtOH is still present on the glass substrate that can diminish binding affinity of Ad for the $\beta$-CD cavity. 
a)

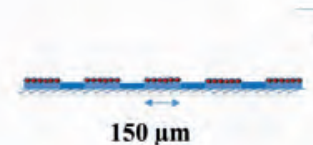

Sonication in $\mathrm{EtOH} / \mathrm{H}_{2} \mathrm{O}$
Addition of PS-Cy3 and PS-Cy5.5-Ad

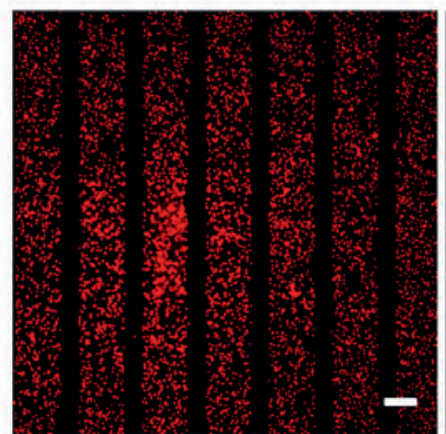

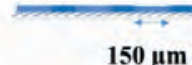

$150 \mu \mathrm{m}$

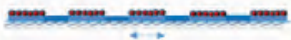

$150 \mu \mathrm{m}$
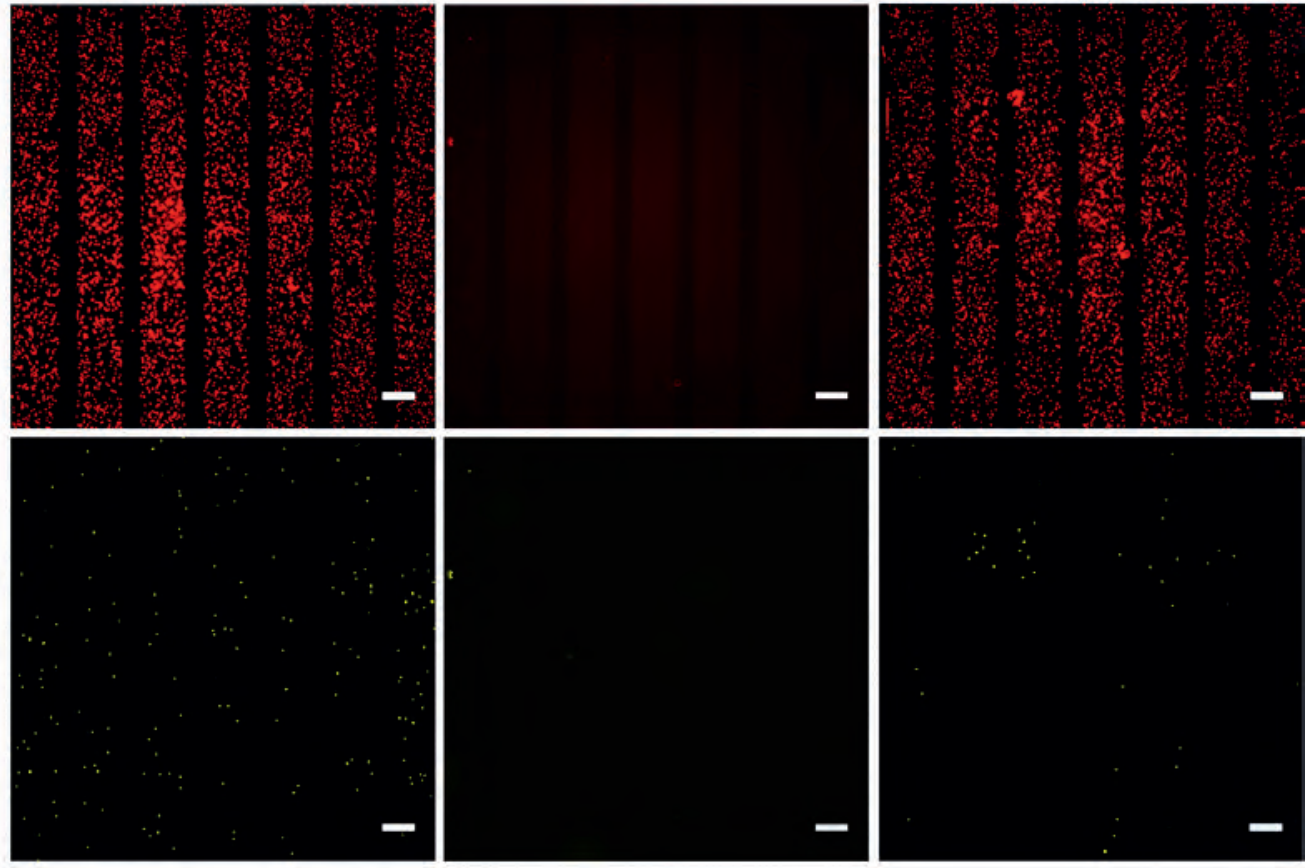

b)

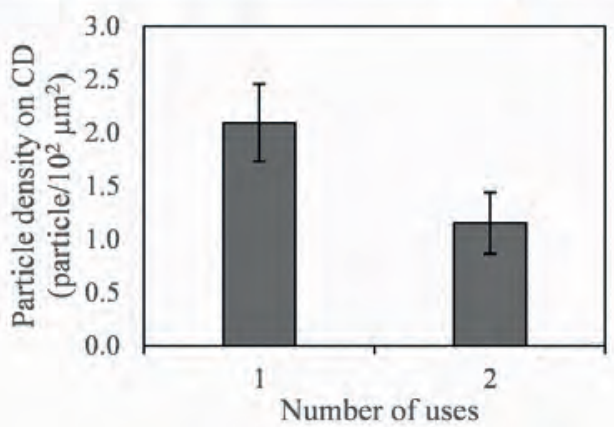

Fig. 4.6: a) Fluorescence microscope images of PS particles adhered to $\beta$-CD lines and subsequent removal and readdition of PS particles at $5 \mu \mathrm{L} / \mathrm{min}$ flow rate. Cy5 channels (red) shows PS-Cy5.5-Ad particles and RHOD channel (yellow) shows PS-Cy3 particles. b) Particle density on $\beta$-CD after multiple uses. 


\subsection{Conclusion}

A chemically modified glass platform has been developed that can capture micrometer sized particles on flow in a recyclable manner. The capture of 'large' particles, about $10^{3}$ times larger than the host molecule $\beta-\mathrm{CD}$, was possible through multivalent host-guest interactions between adamantane and cyclodextrin. Immobilization of adamantane functionalized PS particles to $\beta-\mathrm{CD}$ modified platforms was flow rate dependent, with an optimal capturing density at 2.5 to $5 \mu \mathrm{L} / \mathrm{min}$. Changing the degree of adamantane functionalization on the PS particles influenced the degree of binding on the glass surfaces, underpinning the effect of multivalent, host-guest interactions on particle binding. The modified glass platforms could also be recycled, highlighting the potential of using such systems in water purification set-ups. The depicted results of this model system show that host-guest interactions can bridge the gap between nano- and microscale, and also give insight on certain parameters and hurdles that are important to take into account for approaching the application of bacterial cell targeting in waste-water. Moreover, the experimental setup of this model system can easily be used for immobilization tests with different types of molecules or particles. 


\subsection{References}

1. Mrksich, M.; Whitesides, G. M. Using Self-Assembled Monolayers to Understand the Interactions of Man-made Surfaces with Proteins and Cells. Annual Review of Biophysics and Biomolecular Structure 1996, 25 (1), 55-78.

2. Anselme, K.; Davidson, P.; Popa, A. M.; Giazzon, M.; Liley, M.; Ploux, L. The Interaction of Cells and Bacteria with Surfaces Structured at the Nanometre Scale. Acta Biomaterialia 2010, 6 (10), 3824-3846.

3. Torkelson, A. A.; da Silva, A. K.; Love, D. C.; Kim, J. Y.; Alper, J. P.; Coox, B.; Dahm, J.; Kozodoy, P.; Maboudian, R.; Nelson, K. L. Investigation of quaternary ammonium silanecoated sand filter for the removal of bacteria and viruses from drinking water. Journal of Applied Microbiology 2012, 113 (5), 1196-1207.

4. Shtarker-Sasi, A.; Castro-Sowinski, S.; Matan, O.; Kagan, T.; Nir, S.; Okon, Y.; Nasser, A. M. Removal of bacteria and Cryptosporidium from water by micelle-montmorillonite complexes. Desalination and Water Treatment 2013, 51 (40-42), 7672-7680.

5. Hagen, K. Removal of Particles, Bacteria and Parasites with Ultrafiltration for Drinking Water Treatment. Desalination 1998, 119 (1), 85-91.

6. MacRae, I. C.; Evans, S. K. Removal of bacteria from water by adsorption to magnetite. Water Research 1984, 18 (11), 1377-1380.

7. Krasowska, A.; Sigler, K. How Microorganisms use Hydrophobicity and what does this mean for Human Needs? Frontiers in Cellular and Infection Microbiology 2014, 4, 112.

8. Mrksich, M.; Dike, L. E.; Tien, J.; Ingber, D. E.; Whitesides, G. M. Using Microcontact Printing to Pattern the Attachment of Mammalian Cells to Self-Assembled Monolayers of Alkanethiolates on Transparent Films of Gold and Silver. Exp Cell Res 1997, 235 (2), 305-313.

9. Mrksich, M.; Chen, C. S.; Xia, Y.; Dike, L. E.; Ingber, D. E.; Whitesides, G. M. Controlling cell attachment on contoured surfaces with self-assembled monolayers of alkanethiolates on gold. Proceedings of the National Academy of Sciences of the United States of America 1996, 93 (20), 10775-10778.

10. Kumar, A.; Biebuyck, H. A.; Whitesides, G. M. Patterning Self-Assembled Monolayers: Applications in Materials Science. Langmuir 1994, 10 (5), 1498-1511.

11. Ostuni, E.; Yan, L.; Whitesides, G. M. The interaction of proteins and cells with selfassembled monolayers of alkanethiolates on gold and silver. Colloids and Surfaces B: Biointerfaces 1999, 15 (1), 3-30.

12. Kumar, A.; Whitesides, G. M. Patterned Condensation Fig. s as Optical Diffraction Gratings. Science 1994, 263 (5143), 60.

13. Xia, Y.; Whitesides, G. M. Soft Lithography. Angewandte Chemie International Edition 1998, 37 (5), 550-575.

14. Voskuhl, J.; Sankaran, S.; Jonkheijm, P. Optical control over bioactive ligands at supramolecular surfaces. Chemical Communications 2014, 50 (96), 15144-15147.

15. Sankaran, S.; van Weerd, J.; Voskuhl, J.; Karperien, M.; Jonkheijm, P. Photoresponsive Cucurbit[8]uril-Mediated Adhesion of Bacteria on Supported Lipid Bilayers. Small 2015, 11 (46), 6187-6196.

16. Sankaran, S.; Kiren, M. C.; Jonkheijm, P. Incorporating Bacteria as a Living Component in Supramolecular Self-Assembled Monolayers through Dynamic Nanoscale Interactions. ACS Nano 2015, 9 (4), 3579-3586. 
17. Di Iorio, D.; Verheijden, M. L.; van der Vries, E.; Jonkheijm, P.; Huskens, J. Weak Multivalent Binding of Influenza Hemagglutinin Nanoparticles at a Sialoglycan-Functionalized Supported Lipid Bilayer. ACS Nano 2019, 13 (3), 3413-3423.

18. Szejtli, J. Introduction and General Overview of Cyclodextrin Chemistry. Chemical Reviews 1998, 98 (5), 1743-1754.

19. Loftsson, T.; Brewster, M. E. Pharmaceutical Applications of Cyclodextrins. 1. Drug Solubilization and Stabilization. Journal of Pharmaceutical Sciences 1996, 85 (10), 1017-1025.

20. Park, C.; Kim, H.; Kim, S.; Kim, C. Enzyme Responsive Nanocontainers with Cyclodextrin Gatekeepers and Synergistic Effects in Release of Guests. Journal of the American Chemical Society 2009, 131 (46), 16614-16615.

21. Onclin, S.; Mulder, A.; Huskens, J.; Ravoo, B. J.; Reinhoudt, D. N. Molecular printboards: monolayers of beta-cyclodextrins on silicon oxide surfaces. Langmuir 2004, 20 (13), 5460-5466.

22. Ludden, M. J. W.; Reinhoudt, D. N.; Huskens, J. Molecular Printboards: Versatile Platforms for the Creation and Positioning of Supramolecular Assemblies and Materials. Chemical Society Reviews 2006, 35 (11), 1122-1134.

23. Gonzalez-Campo, A.; Hsu, S. H.; Puig, L.; Huskens, J.; Reinhoudt, D. N.; Velders, A. H. Orthogonal covalent and noncovalent functionalization of cyclodextrin-alkyne patterned surfaces. Journal of the American Chemical Society 2010, 132 (33), 11434-11436.

24. Krieg, E.; Bastings, M. M. C.; Besenius, P.; Rybtchinski, B. Supramolecular Polymers in Aqueous Media. Chemical Reviews 2016, 116 (4), 2414-2477.

25. de laRica, R.; Fratila, R. M.; Szarpak, A.; Huskens, J.; Velders, A. H. Multivalent Nanoparticle Networks as Ultrasensitive Enzyme Sensors. Angewandte Chemie International Edition 2011, 50 (25), 5704-5707.

26. Mahalingam, V.; Onclin, S.; Peter, M.; Ravoo, B. J.; Huskens, J.; Reinhoudt, D. N. Directed Self-Assembly of Functionalized Silica Nanoparticles on Molecular Printboards through Multivalent Supramolecular Interactions. Langmuir 2004, 20 (26), 11756-11762.

27. Dorokhin, D.; Hsu, S.-H.; Tomczak, N.; Reinhoudt, D. N.; Huskens, J.; Velders, A. H.; Vancso, G. J. Fabrication and Luminescence of Designer Surface Patterns with $\beta$-Cyclodextrin Functionalized Quantum Dots via Multivalent Supramolecular Coupling. ACS Nano 2010, 4 (1), 137-142.

28. Harada, A.; Kobayashi, R.; Takashima, Y.; Hashidzume, A.; Yamaguchi, H. Macroscopic self-assembly through molecular recognition. Nature Chemistry 2010, 3, 34.

29. Appel, J.; Akerboom, S.; Fokkink, R. G.; Sprakel, J. Facile One-Step Synthesis of Monodisperse Micron-Sized Latex Particles with Highly Carboxylated Surfaces. Macromoleculs Rapid Communications 2013, 34 (16), 1284-1288.

30. Bangs Laboratories, I. Covalent Coupling, TechNote 20, . 2002; pp 1-9.

31. Hen, J. Determination of Surface Carboxyl Groups in Styrene/Itaconic Acid Copolymer Latexes. Journal of Colloid and Interface Science 1974, 49 (3), 425-432.

32. Rood, M. T. M.; Spa, S. J.; Welling, M. M.; ten Hove, J. B.; van Willigen, D. M.; Buckle, T.; Velders, A. H.; van Leeuwen, F. W. B. Obtaining Control of Cell Surface Functionalizations via Pre-Targeting and Supramolecular Host-Guest Interactions. Scientific Reports 2017, 7, 39908.

33. Mulder, A.; Onclin, S.; Peter, M.; Hoogenboom, J. P.; Beijleveld, H.; ter Maat, J.; Garcia-Parajo, M. F.; Ravoo, B. J.; Huskens, J.; van Hulst, N. F.; Reinhoudt, D. N. Molecular Printboards on Silicon Oxide: Lithographic Patterning of Cyclodextrin Monolayers with Multivalent, Fluorescent Guest Molecules. Small 2005, 1 (2), 242-253. 
34. Carrazana, J.; Jover, A.; Meijide, F.; Soto, V. H.; Vázquez Tato, J. Complexation of Adamantyl Compounds by $\beta$-Cyclodextrin and Monoaminoderivatives. The Journal of Physical Chemistry B 2005, 109 (19), 9719-9726.

35. Schaefer, D. M.; Carpenter, M.; Gady, B.; Reifenberger, R.; Demejo, L. P.; Rimai, D. S. Surface Goughness and its Influence on Particle Adhesion using Atomic Force Techniques. Journal of Adhesion Science and Technology 1995, 9 (8), 1049-1062.

36. Auletta, T.; de Jong, M. R.; Mulder, A.; van Veggel, F. C.; Huskens, J.; Reinhoudt, D. N.; Zou, S.; Zapotoczny, S.; Schönherr, H.; Vancso, G. J. $\beta$-Cyclodextrin Host- Guest Complexes Probed under Thermodynamic Equilibrium: Thermodynamics and AFM Force Spectroscopy. Journal of the American Chemical Society 2004, 126 (5), 1577-1584.

37. Razaghi, R.; Saidi, M. H. Transportation and Settling Distribution of Microparticles in Low-Reynolds-Number Poiseuille Flow in Microchannel. Journal of Dispersion Science and Technology 2015, 37 (4), 582-594.

38. Harrison, J. C.; Eftink, M. R. Cyclodextrin-Adamantanecarboxylate Inclusion Complexes: A Model System for the Hydrophobic Effect. Biopolymers: Original Research on Biomolecules 1982, 21 (6), 1153-1166. 


\subsection{Supplementary Figures}

\section{Structures}

Cy3
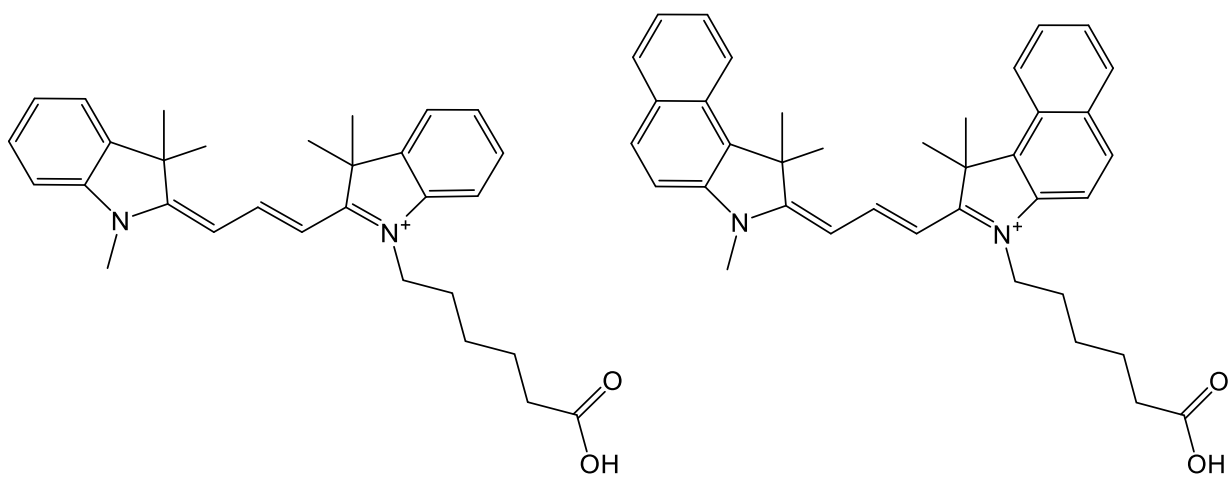

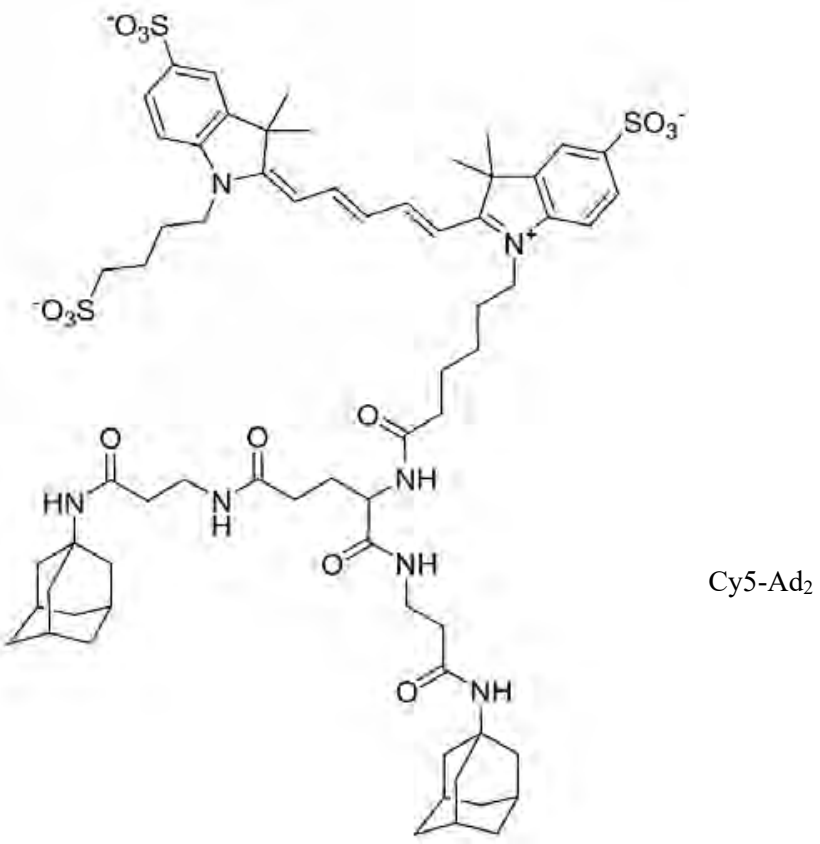

Fig. S1: Structures of dye molecules used. Cy3 and Cy5.5 were used to stain the PS particles. Cy5- $\mathrm{Ad}_{2}$ was used for labelling $\beta-C D$ patterns on glass slides 


\section{Characterization of PS particles}

DLS measurements were carried out after the synthesis of fluorescent PS particles and functionalization with adamantane in order to validate their size. According to the protocol from Appel et al.,the reported size with DLS was $1038 \mathrm{~nm}$ for pSIA25 latex particles ${ }^{1}$. Fig. S1 shows that the size of synthesized PS particles is around this value. The depicted results are averages from 3 repeat measurements and the concentration of samples is $60 \mathrm{fM}$.

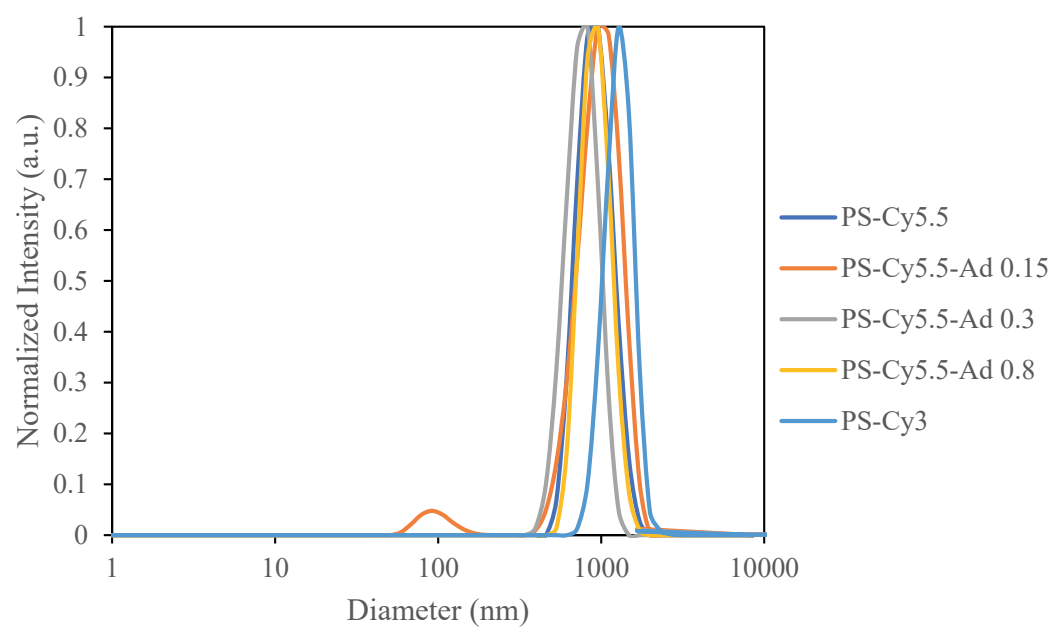

Fig. S2: DLS plot of PS particles showing size vs normalized intensity of the different PS particles 


\section{Dimensions of Micronit ${ }^{\circledR}$ flow cell and Comsol flow channel}

a

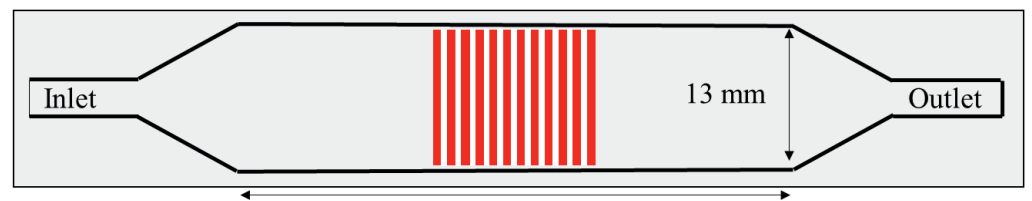

$20 \mathrm{~mm}$

b

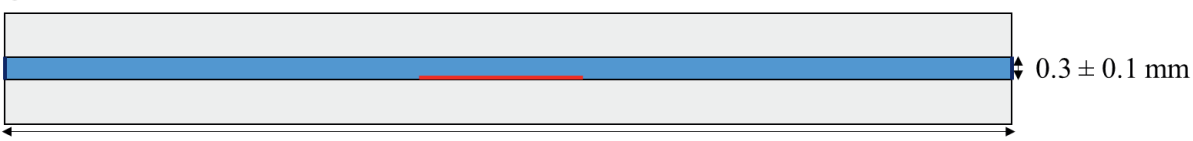

$41 \mathrm{~mm}$

$\mathrm{c}$ $0.3 \mathrm{~mm}$

$20 \mathrm{~mm}$

Fig. S3: Scheme showing the dimensions of an extended 4515 resealable flow cell purchased from Micronit ${ }^{\circledR}$. a) Top view of the flow cell and b) side view of flow cell showing casket slide on top of an 'empty' slide. The bottom slide is functionalized with patterns of $\beta-C D$ approximately in the center over a surface area of $10 \times 10 \mathrm{~mm}^{2}$ (marked in red). c) Dimensions of the flow channel used in Comsol showcasing the area (in red) where particles were counted for the quantitative analysis. 


\section{Characterization of $\beta-C D$ printed Glass Surfaces}

In order to show successful patterning of $\beta-C D$, a validation step using a diadamantane functionalized Cy5 dye (Cy5-Ad2, structure shown in Fig. S1) was implemented. A glass slide microcontact printed with $\beta-\mathrm{CD}$ was placed upside down on $0.28 \mu \mathrm{M}$ of $\mathrm{Cy} 5-\mathrm{Ad}_{2}$ in $1 \mathrm{x}$ PBS and incubated for 15 minutes. The glass slide was then thoroughly rinsed with DI water and dried with nitrogen before imaging on the fluorescence microscope. As observed in Fig. S4, $\mathrm{CD}$ patterns are filled with $\mathrm{Cy} 5-\mathrm{Ad}_{2}$ molecules through host-guest interactions.

a)

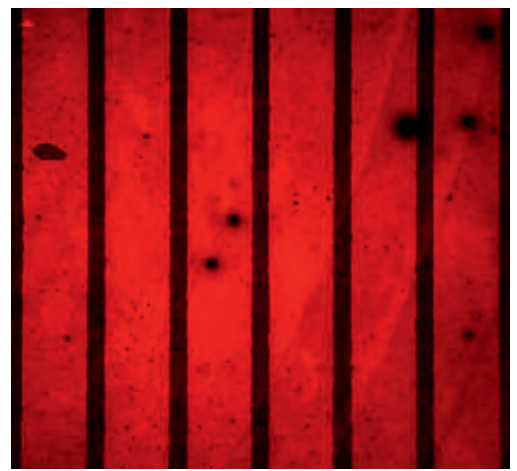

b)

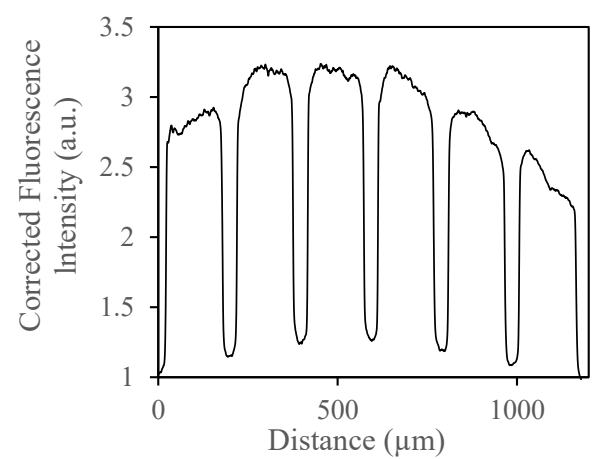

Fig. S4: a) Fluorescence image of $\mathrm{Cy} 5-\mathrm{Ad}_{2}$ adhered to $\beta-\mathrm{CD}$ patterns. b) Fluorescence intensity profile of a). 


\section{Determining PS particle concentration}

The concentration of PS particles was determined through freeze drying the PS emulsion after synthesis and washing the particles with DI water. 3 glass vials were weighed and $5 \mathrm{~mL}$ of undiluted PS particles was pipetted into each. After freeze drying the vials were weighed again to determine the mass of the dried PS particle emulsion. From the mass concentration $(\mathrm{g} / \mathrm{mL})$ the amount of particles per $\mathrm{mL}$ can be calculated from the following equation:

$$
\text { Eq. S1: number of particles per } \mathrm{mL}=\frac{6 W \times 10^{12}}{\rho \times \pi \times \sigma^{3}}
$$

Where $\mathrm{W}$ is the grams of polymer per $\mathrm{mL}$ in latex, $\rho$ is the density of polymer in grams per $\mathrm{mL}$ ( 1.05 for polystyrene) and $\sigma$ is the diameter in microns of polystyrene particles. This equation is derived from dividing mass concentration by the density and from calculating the volume of a sphere. The ' 6 ' and ' $10^{12}$ ' are placed there for simplification and unit conversions. W was determined at $0.100 \pm 0.006 \mathrm{~g} / \mathrm{mL}$ as the average mass concentration from the freeze dried samples. This is equal to $1.82 \times 10^{11}$ particles $/ \mathrm{mL}$. According to literature, ${ }^{1}$ the PS particles have a $\mathrm{CO}_{2} \mathrm{H}$ parking area of $0.14 \mathrm{~nm}^{2}$, which results in a $\mathrm{CO}_{2} \mathrm{H}$ concentration of $6.78 \mathrm{mM}$.

Table S1: PS particle characterization based on radius and literature $\mathrm{COOH}$ parking area

\section{PS particle characterization}

Particle radius $(\mu \mathrm{m})$

Surface area $\left(\mu \mathrm{m}^{2}\right)$

Theoretical/Literature parking area per $\mathrm{CO}_{2} \mathrm{H}\left(\mathrm{nm}^{2}\right)$

Theoretical $\mathrm{CO}_{2} \mathrm{H}$ groups on surface

$\mathrm{W}(\mathrm{g} / \mathrm{mL})$ (Freeze Drying results)

Particles per $\mathrm{mL}$ $1.82 \times 10^{11}$

Particles per L $1.82 \times 10^{14}$ 


\section{Reverse conductometric titration of PS particles}

Quantification of the amount of acid groups on the PS particle surface was carried out using reverse conductometric titration. ${ }^{2}$ The $\mathrm{pH}$ of the solution was first increased to $\mathrm{pH} 10 \mathrm{in}$ order to de-protonate all the $\mathrm{CO}_{2} \mathrm{H}$ groups and then titration with $\mathrm{HCl}$ was carried out. The first stage of the graph corresponds to titration of strong base, the second stage to the titration carboxylate ions $\left(\mathrm{CO}_{2}^{-}\right)$on the PS surface and the third one is due to increase in the $\mathrm{HCl}$ concentration.

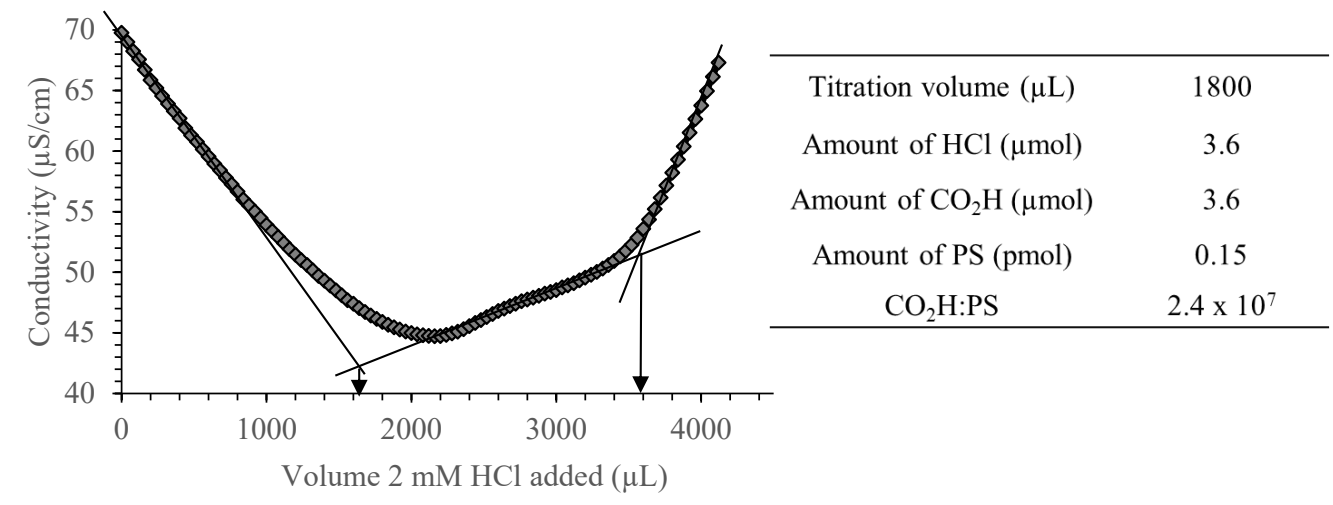

Fig. S5: Conductometric titration of PS-Cy5.5 particles with $\mathrm{HCl}$ after deprotonation with $\mathrm{NaOH}$. First arrow marks start of the titration of $\mathrm{CO}_{2} \mathrm{H}$ groups on PS and second arrow the end of the titration. Table on the right shows determination of amount of $\mathrm{CO}_{2} \mathrm{H}$ groups on the particles from the titration volume 


\section{NMR analysis of supernatants from PS-Ad particles}

To quantify the amount of $\mathrm{Ad}-\mathrm{NH}_{2}$ that was conjugated to the particles, proton NMR analysis of the supernatant after EDC coupling was carried out. A reference spectrum of $10 \mathrm{mM}$ Ad- $\mathrm{NH}_{2}$ was measured to identify characteristic peaks for adamantane and determine the integral of peaks compared to the internal standard (1 mM TMSP). This internal standard is calibrated for 9 protons. The peak at $\delta=1.64-1.57 \mathrm{ppm}$ is attributed to 3 protons from adamantane, therefore the acquired integral from spectra (after calibration with the TMSP internal standard) was divided by 3 to give the concentration in $\mathrm{mM}$ of $\mathrm{Ad}-\mathrm{NH}_{2}$ left in the supernatant. This was then converted to $\mu \mathrm{mol}$ based on the $1 \mathrm{~mL} \mathrm{D}_{2} \mathrm{O}$ the dried supernatant was dissolved in.

For determining the non-specific uptake of $\mathrm{Ad}-\mathrm{NH}_{2}$ in the dispersion of PS particles, EDC was omitted during Ad- $\mathrm{NH}_{2}$ coupling for all the concentrations of $\mathrm{Ad}-\mathrm{NH}_{2}$ used (Table S2). Non-specific uptake was then calculated as a percentage by comparing the $\mathrm{Ad}-\mathrm{NH}_{2}$ amount left in the PS particle suspensions. 


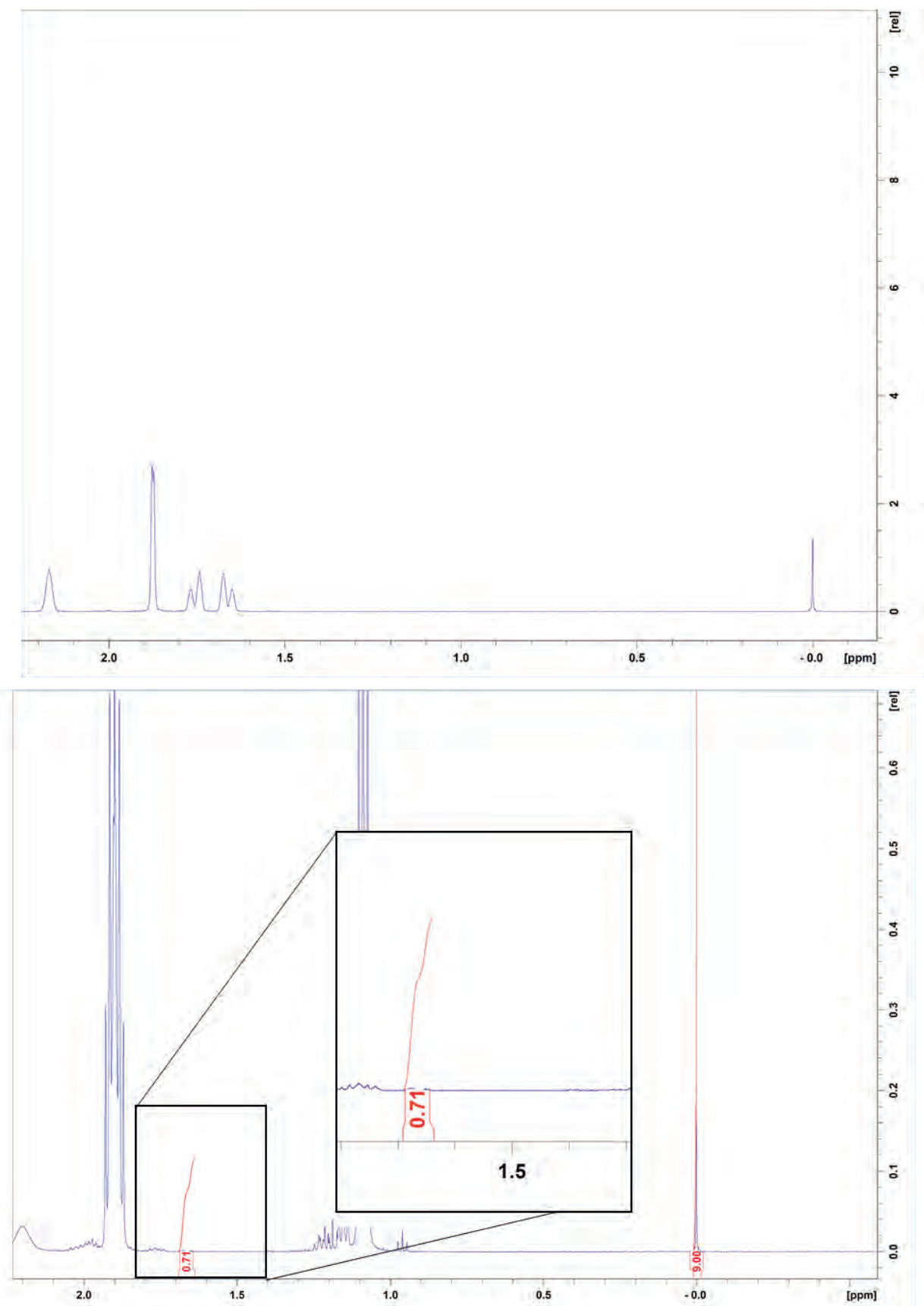

Fig. S6: NMR spectra of adamantane amine reference (top) and supernatant of $0.1 \mathrm{mM}$ (bottom) $\mathrm{Ad}-\mathrm{NH}_{2} \mathrm{PS}$ sample 

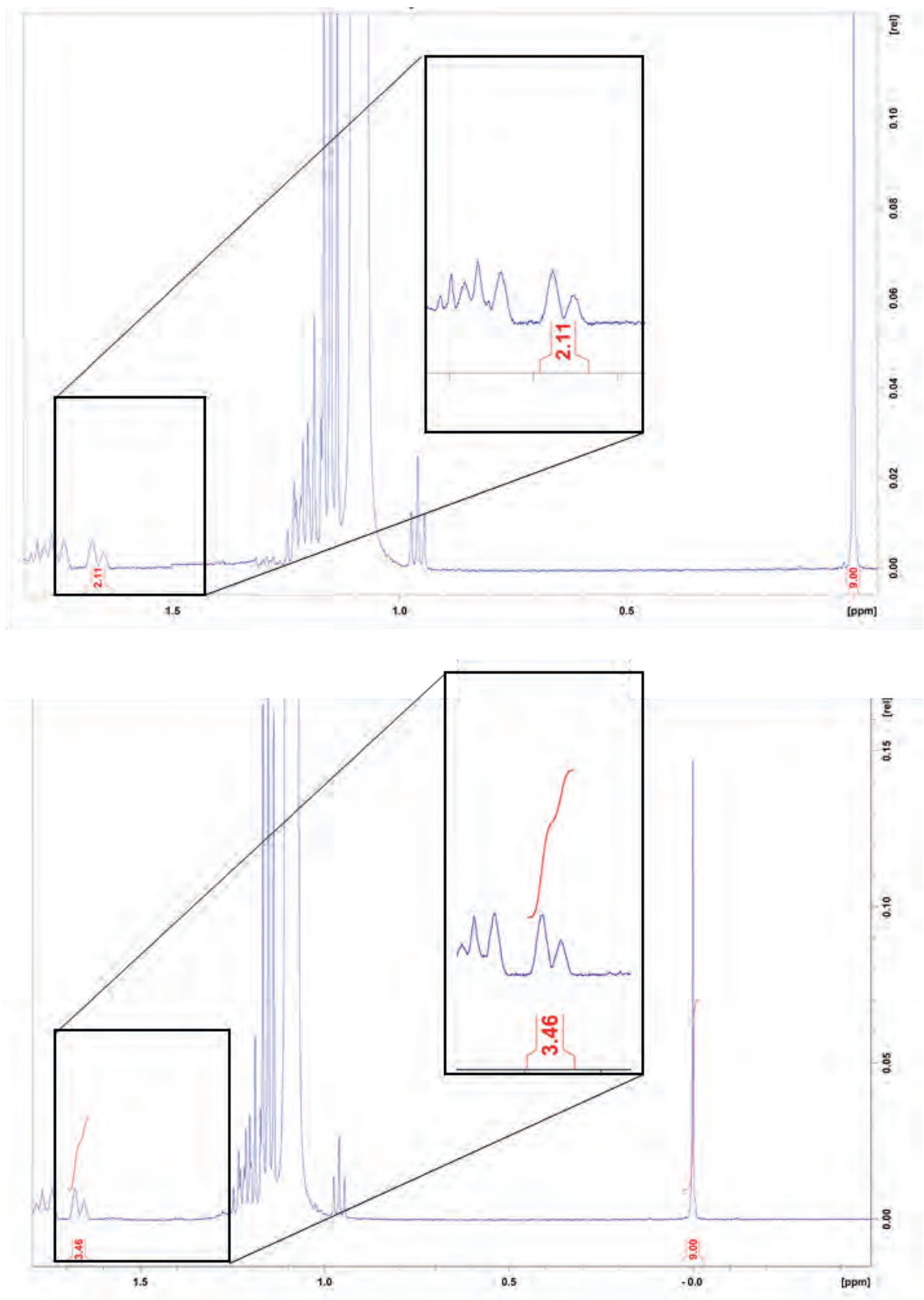

Fig. S7: NMR spectra of 0.25 (top) and $0.5 \mathrm{mM}$ (bottom) Ad- $\mathrm{NH}_{2}$ PS supernatant samples 
Table S2: Determining uptake of $\mathrm{Ad}-\mathrm{NH}_{2}$ in PS particles without EDC for the different amounts of $\mathrm{Ad}-\mathrm{NH}_{2}$ added

\begin{tabular}{|c|c|c|c|}
\hline $\begin{array}{l}\text { PS-Cy5.5-Ad control } \\
\text { samples without EDC }\end{array}$ & PS-Cy5.5-0.15Ad & PS-Cy5.5-0.3Ad & PS-Cy5.5-0.8Ad \\
\hline $\begin{array}{l}\text { Ad-NH} 2 \text { added to PS } \\
(\mu \mathrm{mol})\end{array}$ & 0.53 & 1.33 & 2.67 \\
\hline $\begin{array}{l}\text { Ad-NH} 2 \text { amount in } \\
\text { supernatant }(\mu \mathrm{mol})\end{array}$ & 0.37 & 1.07 & 2.15 \\
\hline $\begin{array}{c}\text { Ad-NH} 2 \text { in PS } \\
\text { suspension }(\mu \mathrm{mol})\end{array}$ & 0.16 & 0.26 & 0.52 \\
\hline $\begin{array}{l}\text { Ad- } \mathrm{NH}_{2} \text { with EDC in } \\
\text { PS suspension }(\mu \mathrm{mol})\end{array}$ & 0.29 & 0.63 & 1.52 \\
\hline $\begin{array}{c}\text { Non-specific uptake of } \\
\text { Ad-NH }\end{array}$ & $55 \%$ & $40 \%$ & $34 \%$ \\
\hline
\end{tabular}


Stokes drag force, Reynolds numbers, Péclet number of particles at different flow rates

\section{Stokes law}

$$
\text { Eq. S1: } \quad F_{d}=6 \pi \eta r v
$$

where $F_{d}$ is Stokes Drag $(N), \eta$ is dynamic viscosity $\left(\mathrm{kg} \mathrm{m}^{-1} \mathrm{~s}^{-1}, 0.01\right.$ for water $), \mathrm{r}$ is the radius of particle $(\mathrm{m})$ and $\mathrm{v}$ is the flow velocity relative to the particle $\left(\mathrm{m} \mathrm{s}^{-1}\right)$

Table S3: Table showing the average Stokes Drag particles experience at different flow rates

\begin{tabular}{cll} 
Flow rate $(\boldsymbol{\mu L} / \mathrm{min})$ & Velocity $(\mathrm{m} / \mathrm{s})$ & Stokes Drag $(\mathrm{N})$ \\
\hline 0.125 & $5.35 \times 10^{-6}$ & $5 \times 10^{-15}$ \\
0.25 & $1.07 \times 10^{-6}$ & $1 \times 10^{-14}$ \\
1.25 & $5.35 \times 10^{-6}$ & $5 \times 10^{-14}$ \\
2.5 & $1.07 \times 10^{-5}$ & $1 \times 10^{-13}$ \\
5 & $2.14 \times 10^{-5}$ & $2 \times 10^{-13}$ \\
10 & $4.27 \times 10^{-5}$ & $4 \times 10^{-13}$ \\
100 & $4.27 \times 10^{-4}$ & $4 \times 10^{-12}$ \\
200 & $8.54 \times 10^{-4}$ & $8 \times 10^{-12}$ \\
\hline
\end{tabular}

\section{Reynolds number}

$$
\text { Eq. S2: } \quad R e=\frac{V D_{h}}{v}
$$

Where $\mathrm{V}$ is average velocity $\left(\mathrm{m} \mathrm{s}^{-1}\right), \mathrm{D}_{\mathrm{h}}$ characteristic length of the flow channel $(\mathrm{m})$ and $\mathrm{v}$ is the kinematic viscosity $\left(\mathrm{m}^{2} \mathrm{~s}^{-1}\right)$. In general, Reynolds numbers below 1 dictate a laminar flow regime. 


\section{Péclet number}

$$
\text { Eq. S3: } \quad P e=\frac{V_{x} R}{D}
$$

where $V_{x}$ is the speed of the particles at a certain height, $R$ is the height from the channel wall $(\mathrm{m})$, and $\mathrm{D}$ is the diffusivity $\left(\mathrm{m}^{2} \mathrm{~s}^{-1}\right)$

$$
\text { Eq. S4: } \quad D=\frac{k T}{6 \pi \eta r}
$$

where $\mathrm{k}$ is Boltzmann's constant, $\mathrm{T}$ is temperature $(\mathrm{K}), \eta$ is the dynamic viscosity $\left(\mathrm{kg} \mathrm{m}^{-1} \mathrm{~s}^{-1}\right)$, and $\mathrm{r}$ is the radius of particle $(\mathrm{m})$.

The diffusivity for the particles is $4.29 \times 10^{-13} \mathrm{~m}^{2} \mathrm{~s}^{-1}$ regardless of the flow rate, while the flow velocity of the particles at $1 \mu \mathrm{m}$ height above the channel wall is 0.0132 times the average flow velocity of particles within the channel.

Table S4: Table showing average flow velocity, Reynolds number, flow velocity at $1 \mu \mathrm{m}$ height above channel wall and Peclet number at $1 \mu \mathrm{m}$ height above channel wall

\begin{tabular}{ccccc}
$\begin{array}{c}\text { Flow rate }(\boldsymbol{\mu L} / \\
\text { min })\end{array}$ & $\begin{array}{c}\text { Average flow } \\
\text { velocity }(\mathbf{m} / \mathbf{s})\end{array}$ & $\begin{array}{c}\text { Reynolds } \\
\text { number }\end{array}$ & $\begin{array}{c}\text { Flow velocity } \\
\text { at } 1 \boldsymbol{\mu m} \text { height }(\mathbf{m} / \mathbf{s})\end{array}$ & $\begin{array}{c}\text { Péclet } \\
\text { number }\end{array}$ \\
\hline 0.125 & $5.35 \times 10^{-6}$ & 0.00025 & $1.42 \times 10^{-8}$ & 0.03 \\
0.25 & $1.07 \times 10^{-6}$ & 0.0005 & $2.84 \times 10^{-8}$ & 0.07 \\
1.25 & $5.35 \times 10^{-6}$ & 0.0025 & $1.42 \times 10^{-7}$ & 0.33 \\
2.5 & $1.07 \times 10^{-5}$ & 0.005 & $2.84 \times 10^{-7}$ & 0.66 \\
5 & $2.14 \times 10^{-5}$ & 0.010 & $5.68 \times 10^{-7}$ & 1.32 \\
10 & $4.27 \times 10^{-5}$ & 0.021 & $1.14 \times 10^{-6}$ & 2.64 \\
100 & $4.27 \times 10^{-4}$ & 0.21 & $1.14 \times 10^{-5}$ & 26.4 \\
200 & $8.54 \times 10^{-4}$ & 0.42 & $2.28 \times 10^{-5}$ & 52.8 \\
\hline
\end{tabular}




\section{Flow of PS particle mix over glycine printed flow cells}

Glycine was microcontact printed on an isothiocyanate coated flow cell similar to $\beta-C D$ glass surface functionalization protocol. The flow cells were also backfilled with PEG. PSCy5.5-0.8Ad and PS-Cy3 particles were flowed over glycine printed flow cells at $5 \mu \mathrm{L} / \mathrm{min}$ for 60 minutes. After flow incubation the flow cell was run dry and the surface was imaged with fluorescence microscopy. The images show no visible patterned binding to the glycine prints on the glass surface from either particle species.
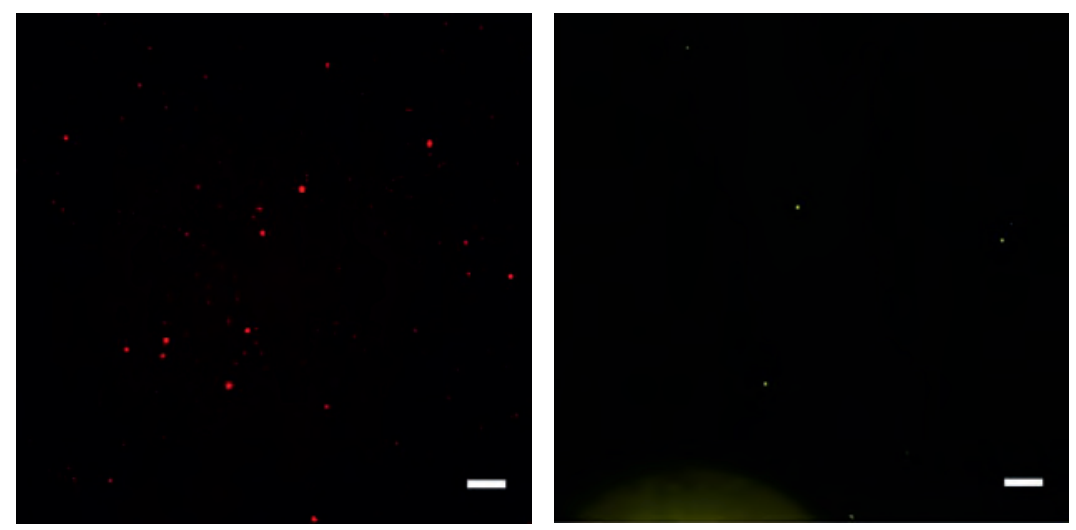

Fig. S8: Microscope images of glycine printed flow cells after addition of PS particles mix at $5 \mu \mathrm{L} / \mathrm{min}$. Left image shows PS-Cy5.5-Ad particles and right images show PS-Cy3 particles 


\section{All flow rate variation experiments for PS-Cy5.5-0.8Ad and PS-Cy3 particles over $\beta$-CD printed surfaces}

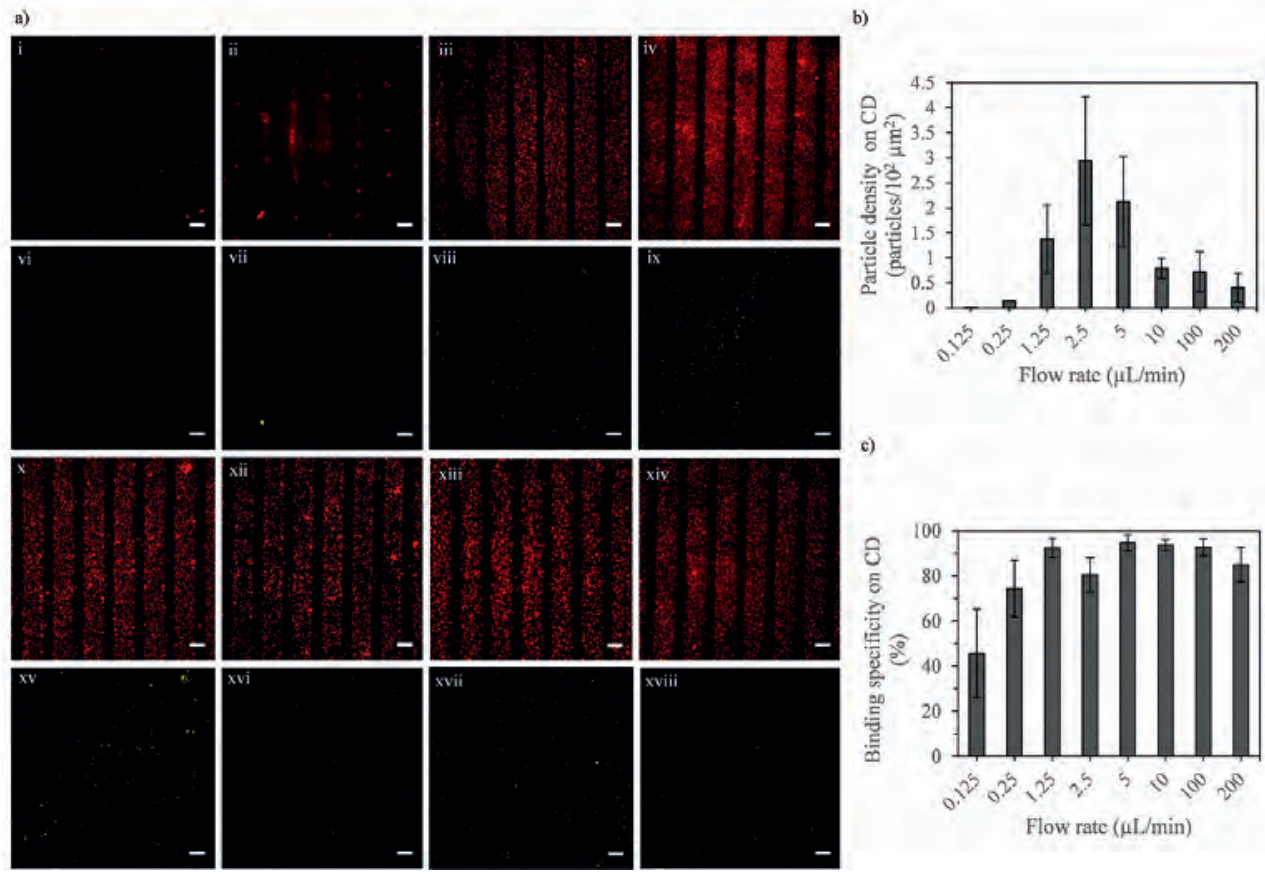

Fig. S9: a) Fluorescence images of $\beta-C D$ platforms after addition of an equimolar mix of fluorescent polystyrene particles PS-Cy5.5-0.8Ad (i-iv, x-xiv) and PS-Cy3 (vi-ix, xv-xvii). Flow rates used are 0.125 (i,vi) 0.25 (ii,vii), 1.25 (iii, xvii), 2.5 (iv, ix), 5 (x,xv), 10 (xii, xvi), 100 (xiii, xvii) and 200 (xiv, xviii) $\mu \mathrm{L} / \mathrm{min}$. Top images are captured with $\mathrm{Cy} 5$ filter and bottom images with RHOD filter at the same location on the surface for the respective flow rate. All scale bars are $100 \mu \mathrm{m}$. b) Graph showing the PS-Cy5.5-Ad particle density on $\beta$-cyclodextrin patterns versus all the flow rates used. c) graph showing the binding specificity for these respective patterns versus the flow rate. 


\section{PS particle distribution after $0.25 \mu \mathrm{L} / \mathrm{min}$ flow rate of 20 hours}
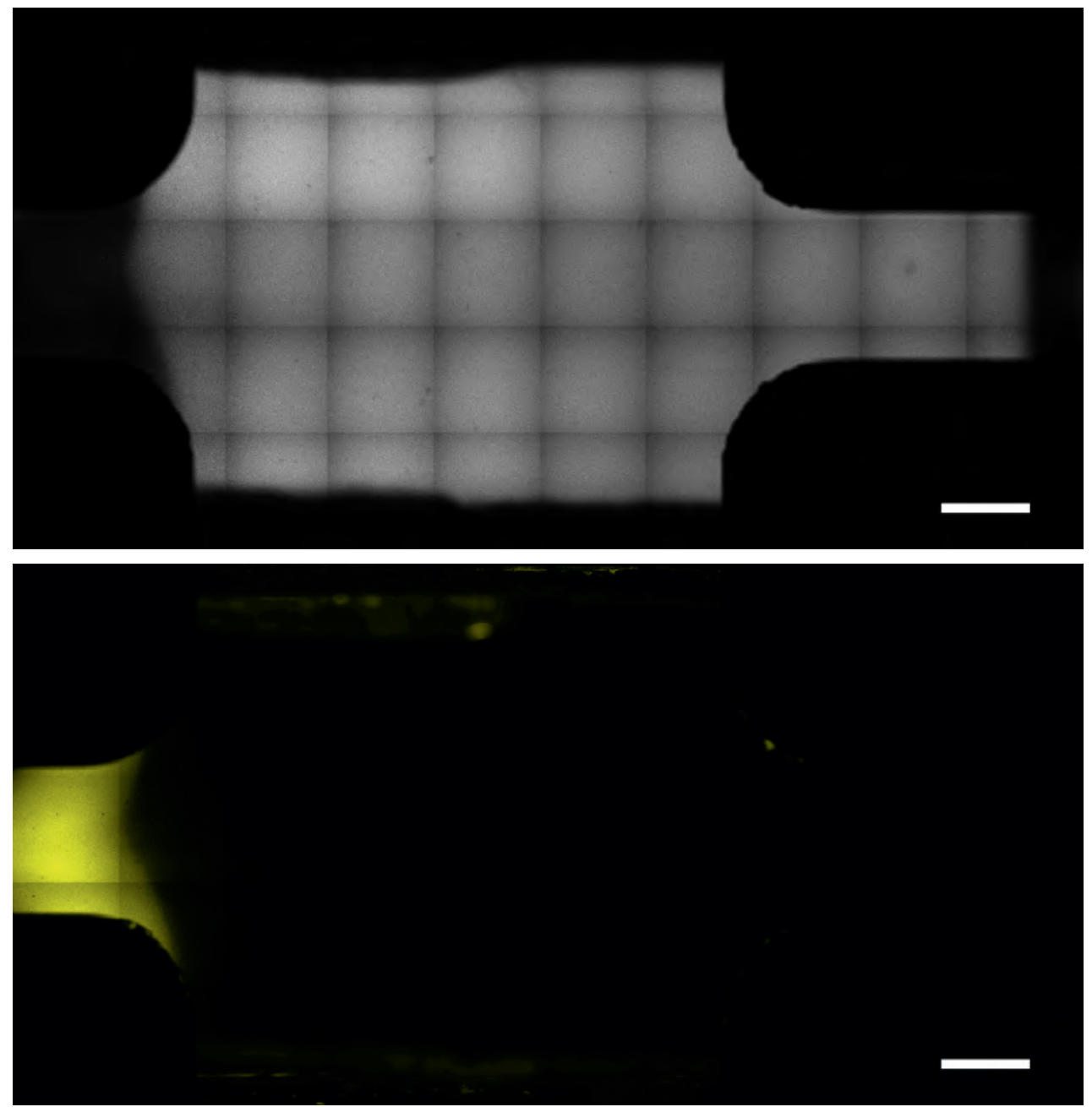

Fig. S10: Overview of extended area (13 mm width) of flow channel after flow incubation of PS-Cy3 particles at $0.25 \mu \mathrm{L} / \mathrm{min}$ for 20 hours over a PEG functionalized glass surface. Captured with $5 \mathrm{x}$ objective and using $10 \mathrm{x} 5$ tilescan. Top image shows the brightfield image while the bottom image shows the RHOD filter. The dark round edges in the corners of the brightfield image are attributed to Micronit flow cell holder, not the flow channel path. Scale bars are $2 \mathrm{~mm}$. 


\section{Distance above the wall for particles at $P e=1$ condition}

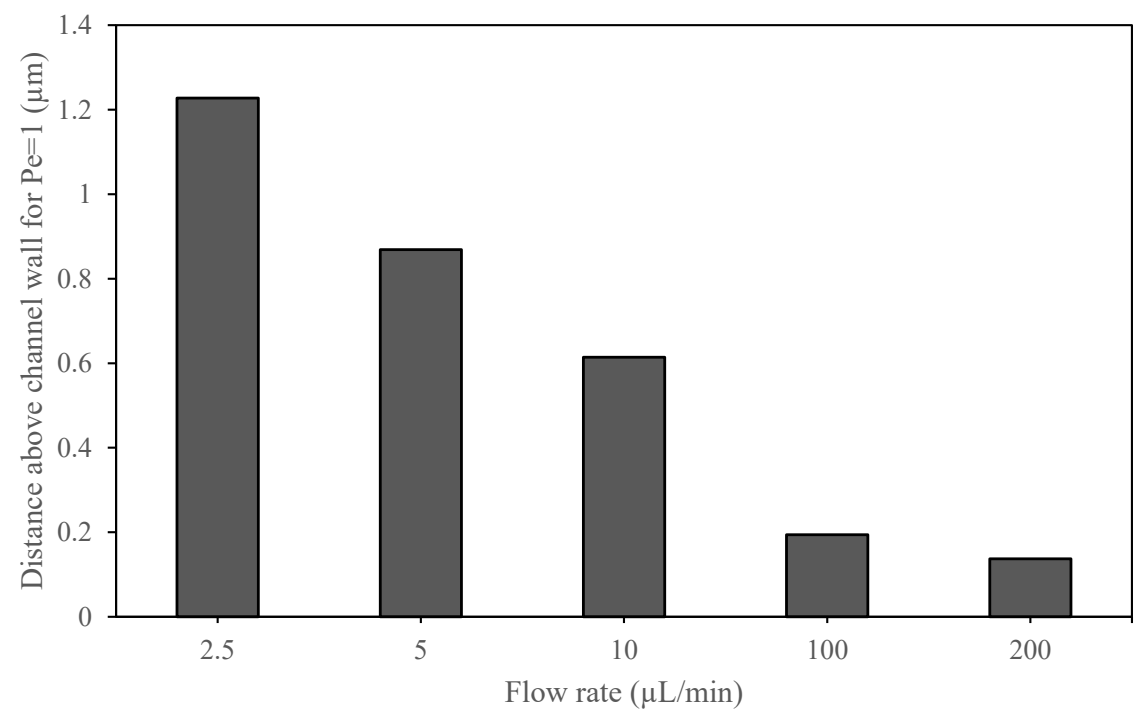

Fig. S11: Graph showing the distance above the wall for the condition that $\mathrm{Pe}=1$ versus the flow rate used.
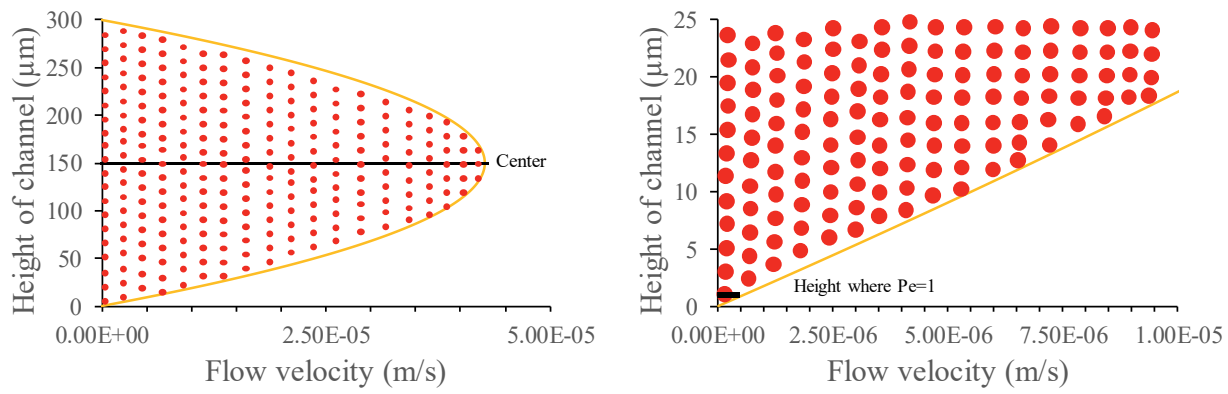

Fig. S12: An example of how particles are spread throughout laminar flow at flow rate $5 \mu \mathrm{L} / \mathrm{min}$ of solution and which part of the solution is most likely to come into contact with the surface based on Peclet number. Graph on the left shows the whole channel height while the graph on the left is zoomed in to showcase the height where $\mathrm{Pe}=1$ 


\section{Quantitative analysis of PS-Cy3 particle density (in PS particle mix) with different}

flow rates of PS particle mix solution and different Ad loadings on PS-Cy5.5-Ad

The nonselective binding of PS-Cy3 particles with the functionalized glass platforms were quantified and compared with different parameters of the flow cell experiments, namely the flow rate used and the Ad loading PS-Cy5.5-Ad. Particle density of PS-Cy3 on $\beta$-CD lines are in general ca 100 times lower than their PS-Cy5.5-Ad counterparts. Nevertheless, an increase in PS-Cy3 particle density is seen with higher Ad loading on PS-Cy5.5-Ad, and a decrease between 2.5 and $200 \mu \mathrm{L} / \mathrm{min}$ flow rate (Fig. S14).
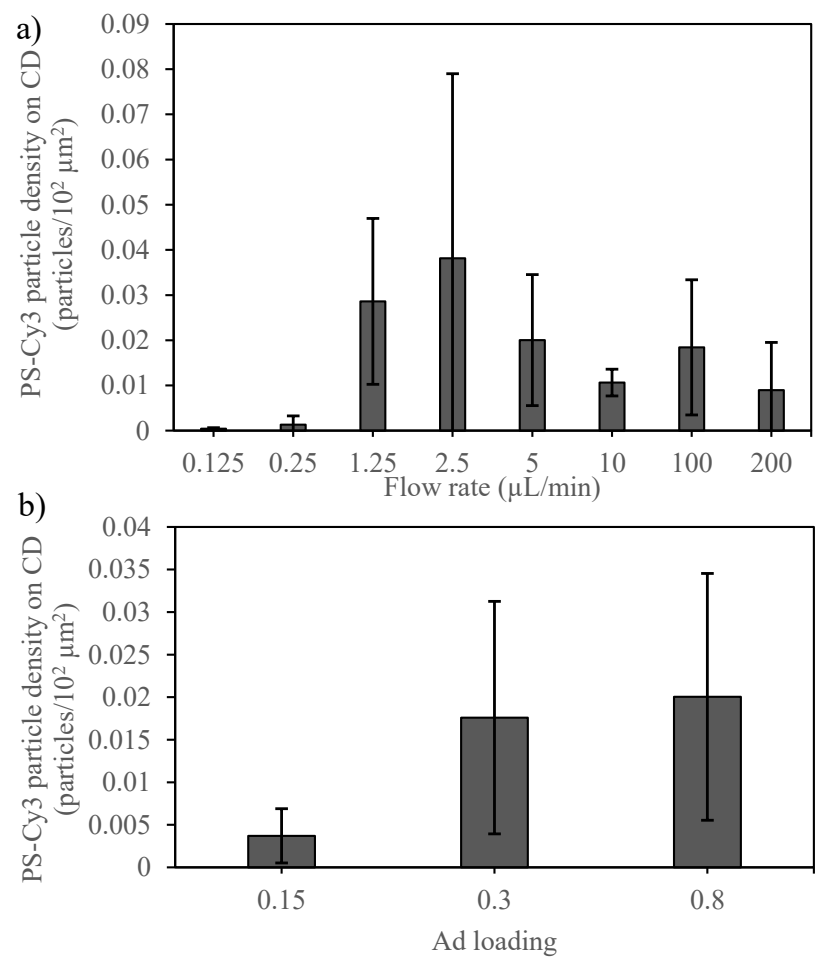

Fig. S13: Graphs showing the effect of PS-Cy3 particle density on $\beta$-CD lines comparied with A) the flow rate of the PS particles mix solution B) the Ad loading on PS-Cy5.5-Ad. 


\section{Flow of PS-Cy3 particles over $\beta-C D$ printed surface}

Non-functionalized PS-Cy3 particles were flowed over CD printed surfaces at a flow rate of 5

$\mu \mathrm{L} /$ min for 60 minutes and then imaged with the fluorescence microscope after running dry. As shown in Fig. S15, no visible patterned binding is observed for the non-functionalized PSCy3 particles when PS-Cy5.5-Ad particles are not present.

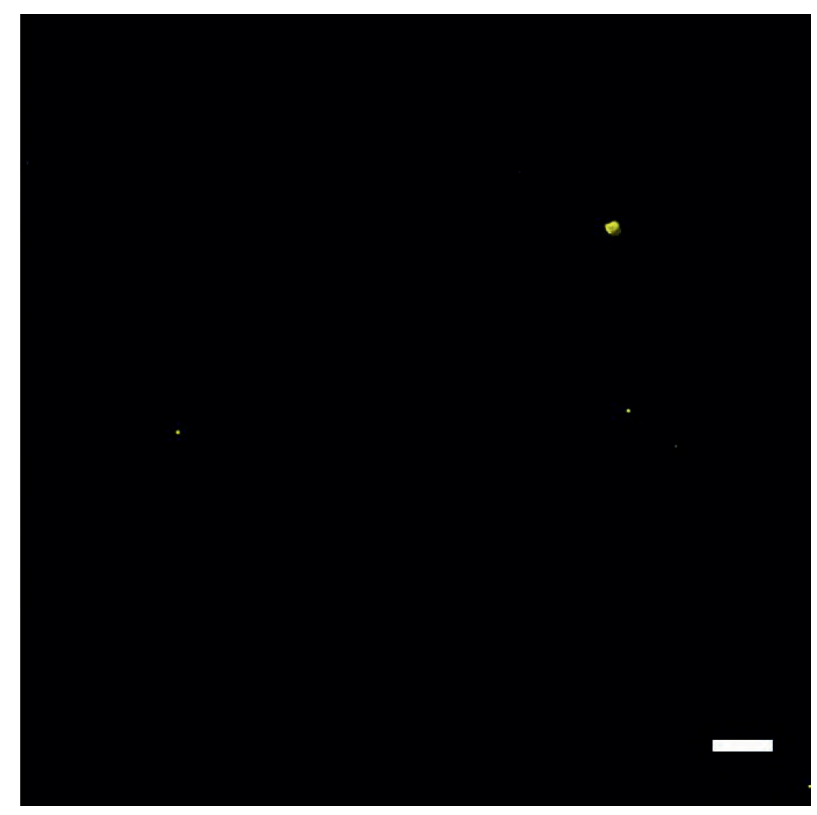

Fig. S14: Fluorescence image of $5 \mu \mathrm{L} / \mathrm{min}$ addition of PS-Cy3 over $\beta$ CD patterns. Scale bar is $100 \mu \mathrm{m}$. 


\section{Chapter 5}

\section{Supramolecular Immobilization of Bacteria in Microfluidic Systems In Presence or Absence of an Adamantane Functionalized Peptide}

Specific detection and targeting of pathogenic bacteria is an important topic within environmental technology. Filters for water treatment applications generally employ adsorption-based techniques, which removes almost all (in)organic material, rather than selectively targeting pathogenic bacteria. This also creates clogging problems with filters. Here, we present a versatile method for studying the supramolecular immobilization of Gram-positive and -negative bacteria (Staphylococcus (S.) aureus and Escherichia (E.) coli, respectively) in microfluidic devices. As proof of concept for using supramolecular host-guest chemistry to realize specific bacterial binding, we used a bacterial membrane-adhering ubiquicidin segment modified with two adamantane (Ad, guest) molecules $\left(\mathrm{UBI}-\mathrm{Ad}_{2}\right)$ to support immobilization on $\beta$-cyclodextrin ( $\beta$-CD, host) patterns. Wild type bacteria attached to both $\beta$-CD patterns and spacing on the glass surface, while UBI-Ad $\mathrm{A}_{2}$ targeted $S$. aureus immobilized with a higher density but more importantly, mainly on $\beta$-CD patterns. When $\beta$-CD patterns on the glass surface were first functionalized with UBI-Ad $\mathrm{Ad}_{2}$ followed by exposure to wild type bacteria, the binding density was further increased and specific immobilization was also observed on patterned areas. Therefore, it is suggested that aspecific interactions with spacing between $\beta$ $\mathrm{CD}$ patterns are blocked when covering the bacterial cell or $\beta-\mathrm{CD}$ functionalized surfaces with UBI-Ad2. In general, a higher amount of $S$. aureus was captured than the E. coli. The acquired results show that specific immobilization of biological entities such as bacteria is possible in small-scale microfluidic systems.

Stan B.J. Willems, Nikolas Duszenko, Clarize M. de Korne, Anton Bunschoten, R. Martijn Wagterveld, Vittorio Saggiomo, Fijs W.B. van Leeuwen, and Aldrik H. Velders. Manuscript in preparation 


\subsection{Introduction}

Infections caused by pathogenic bacteria are still a major cause of mortality worldwide. Some bacterial infections are becoming increasingly difficult to treat due to the emergence of antibiotic resistance, which occurs mostly within waste streams of hospitals and farms. ${ }^{1-3}$ To prevent the spread of bacteria, large scale filters for water treatment are used which function based on adsorption, such as sand or activated carbon. ${ }^{4,5}$ Advanced filters based on micelle complexes and quaternary ammonium have also been researched to assess the removal of bacteria. ${ }^{6,7}$ Importantly, such filters avoid the use of antibiotic treatment and thus reduce potential increase of antibiotic resistance. At the same time, these filters lack specificity for microorganisms or (in)organic material in general, as they do not incorporate molecular recognition type targeting techniques. Therefore, it is of interest to explore if molecular recognition can also be applied to isolate bacteria.

In healthcare, molecular targeting techniques are applied to imaging bacterial infections. The development of antibacterial tracers is based on use of monoclonal antibodies, ${ }^{8-9}$ antibiotics, ${ }^{10,11}$ bacteriophages, ${ }^{12,13}$ and antimicrobial peptides (AMPs). ${ }^{14,15}$ The latter showed the most promise regarding specificity toward bacterial infections compared to nonspecific uptake in, for example, sterile inflammations. ${ }^{16} \mathrm{UBI}_{29-41}$, a synthetic peptide segment (TGRAKRRMQYNRR) derived from ubiquicidin, is a widely used AMP for imaging bacterial infections. ${ }^{16-18}$ In general, AMPs such as $\mathrm{UBI}_{29-41}$ are cationic and therefore bind with negatively charged moieties on the bacterial cell surface, such as lipoteichoic acid, lipopolysaccharides and phospholipids. ${ }^{19}$ Besides charge-mediated interactions, amphipathic interactions with lipids on cell membranes are also responsible for $\mathrm{UBI}_{29-41}$ displaying a higher specificity toward bacteria when compared to other cationic peptides..$^{20,21}$

For capturing bacteria targeted through molecular recognition, supramolecular hostguest chemistry holds promise due to high affinity and non-covalent interactions. ${ }^{22,23}$ Hostguest interactions have also been used for inducing cell-cell interactions between mammalian cells, ${ }^{24}$ or to realize pre-targeting under harsh in vivo conditions. ${ }^{25,26}$ Recently, we translated this concept to the area of water purification. In first instance we used the host-guest interactions to immobilize micro-sized polystyrene constructs functionalized with adamantane (guest) on a 
molecular functionalized planar containing $\beta$-cyclodextrin (host, $\beta$-CD). ${ }^{27}$ To promote performance assessment of such systems, we have developed a method to create polydimethylsiloxane (PDMS)/glass hybrid microfluidic devices as versatile biosensors. ${ }^{28}$ The fabrication of molecular patterns within a biosensor improves analyte detection for quickly establishing signal to background ratio's.

In this study, we integrate the supramolecular $\beta$-CD host surface and unique microfluidic devices systems to test the isolation of bacteria from aqueous streams. By using the molecular targeting capabilities of a diadamantane functionalized $\mathrm{UBI}_{29-41}$ peptide (UBI$\mathrm{Ad}_{2},{ }^{29}$ structure shown in Fig. 5.1b), we functionalize living bacteria cells with adamantane guest molecules, subsequently allowing their immobilization on microfluidic surfaces with supramolecular $\beta$-CD patterns from Chapter 3. Staphylococcus aureus (S. aureus) and Escherichia coli (E. coli) bacteria were used for specific immobilization studies as they represent well-studied models for both Gram-positive and Gram-negative bacteria pathogens. In an attempt to better understand the interaction strengths, we studied how UBI-Ad $\mathrm{Ad}_{2}$ promoted

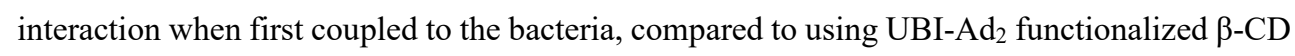
surfaces. 

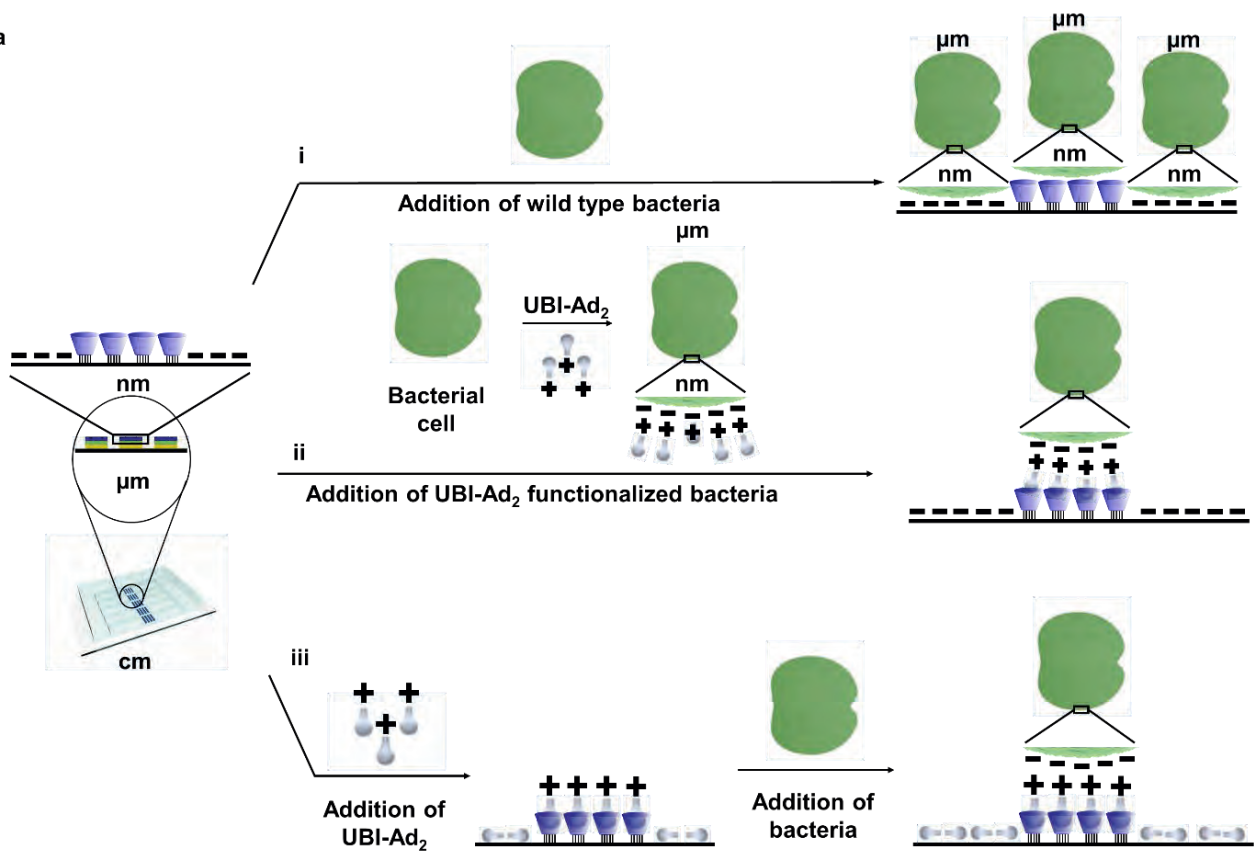

b

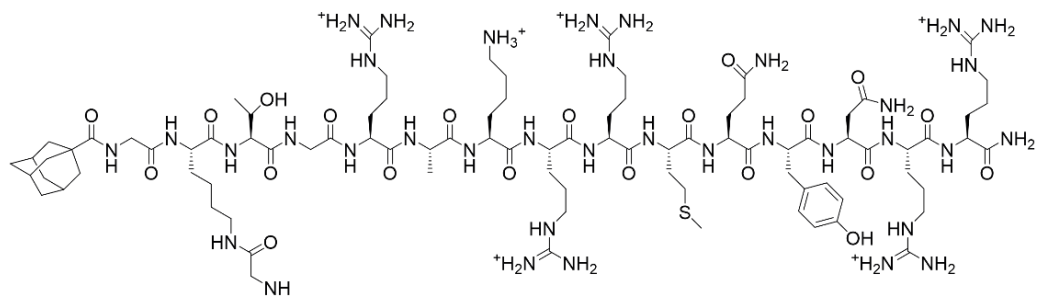

c
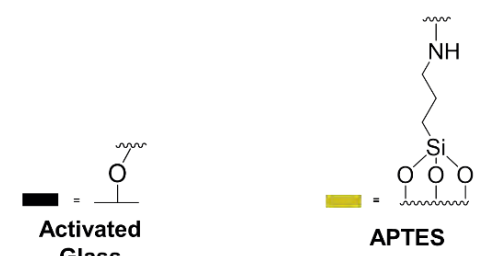

APTES

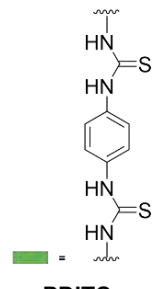

PDITC

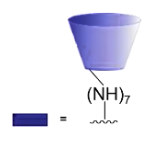

$\beta-C D\left(N_{2}\right)_{7}$

Fig. 5.1: a) Flow experiments with bacteria over $\beta$-CD patterned surface by (i) addition of wild type bacteria over surface as a control (ii) addition of UBI- $\mathrm{Ad}_{2}$ pre-targeted bacteria over $\beta$-CD patterns on glass surface and (iii) pre-functionalization of patterned glass surface with UBI-Ad $\mathrm{Ad}_{2}$ peptide then addition of unaltered bacteria over UBI patterned surface. The different length scales are emphasized to show the range at which interactions are taking place between bacteria, UBI-Ad $\mathrm{Ad}_{2}$ targeting peptide and functionalized $\beta$-CD surfaces. b) Structure of UBI$\mathrm{Ad}_{2}$ peptide used to target bacteria at physiological $\mathrm{pH}$. c) Legend explaining different layers on functionalized surface to yield $\beta-C D$ functionality. 


\subsection{Experimental Section}

\section{General}

Chemicals were purchased from Sigma Aldrich/Merck unless mentioned otherwise. Menzel-Gläser borosilicate glass microscope slides were used to fabricate the microfluidic device and were purchased from Thermo Scientific. Hyper pure polished silicon wafers (76.2 $\mathrm{mm}$ diameter, purchased from University Wafer, USA) were used as silicon masters. A photomask template containing patterns, with different feature and spacing sizes, was designed and ordered from JD photo data (UK). Deionized (DI) water was used in all the experiments. Cyanine 5-diadamantane $\left(\mathrm{Cy} 5-\mathrm{Ad}_{2}\right)$ was synthesized as reported previously. ${ }^{24}$

\section{Instrumentation}

For the preparation of the silicon master for PDMS stamps, a standard UV-lithography set-up was used. $50 \mu \mathrm{m}$ thick SU-8 2025 negative photoresist on the silicon wafer was irradiated with a UV lamp at $\lambda=350 \mathrm{~nm}$ at $50 \%$ intensity. An Inseto Plasma Etch, Inc. PE-25 benchtop air plasma cleaner was used for plasma cycles of $1 \mathrm{~min}$ at its maximum RF plasma power of $100 \mathrm{~W}$ with an air flow of $\sim 10 \mathrm{cc} / \mathrm{min}$, which allowed for a vacuum pressure of 200-250 mTorr within the chamber during plasma treatment. An RC6 Chemistry Hybrid Pump from Vacuubrand (vacuum of $2 \times 10^{-3}$ mbar) was used for applying high vacuum condition in desiccators. The functionalized glass surfaces were imaged using a Leica SP8 confocal laser scanning microscope with 20X magnification objective lens or a Leica DMi8 epi-fluorescence microscope with 10X magnification objective lens. Hoechst stained bacteria were excited $\left(\lambda_{\mathrm{ex}}\right)$ using the $405 \mathrm{~nm}$ laser diode and emission $\left(\lambda_{\mathrm{em}}\right)$ was collected between 420-470 $\mathrm{nm}$. Сy5 emission was measured with the epi-fluorescence microscope at a wavelength $\left(\lambda_{\mathrm{ex}}\right)$ of $590-650$ $\mathrm{nm}$ and emission $\left(\lambda_{\mathrm{em}}\right)$ was collected at $662-738 \mathrm{~nm}$ (using the Y5 filter cube) with an exposure time of $278 \mathrm{~ms}$. 


\section{FIJI/ImageJ data analysis}

The results were analysed with FIJI software (ImageJ). ${ }^{30}$ An automated macro (BiFF_v1.0) was developed which applied a color threshold and a size threshold to the microscopy images $(775 \times 775$ pixels) to segment the bacteria based on their fluorescent signal and size. The user then placed 2 ROIs of the same size ( $735 \times 50$ pixels) to select the $\beta$-CD line and the non- $\beta-C D$ line and calculated the ratio between the bacteria surface found within the 2 ROIs. The fraction of the ROI covered with bacteria was then calculated.

\section{Glass surface functionalization}

The general glass surface functionalization with $\beta$-cyclodextrin was performed as described by Onclin et al..$^{31}$ and creating patterns was carried out through plasma microcontact patterning $(\mathrm{P} \mu \mathrm{CP}){ }^{32}$ In short, glass microscope coverslips of size $56 \times 26 \mathrm{~mm}$ were cleaned and oxidized with piranha solution $\left(\mathrm{H}_{2} \mathrm{SO}_{4}(95-98 \%) / \mathrm{H}_{2} \mathrm{O}_{2}(30 \%)\right.$, 3:1 v/v; Warning! Piranha solutions must be handled with caution as they may unexpectedly detonate) for 45 minutes, rinsed with large amounts DI water, and dried under $\mathrm{N}_{2}$. The glass slides were placed in a high vacuum, pre-heated desiccator together with a glass vial containing $1 \mathrm{~mL}$ of 3aminopropyltriethoxysilane (APTES, 99\%) and placed in an oven at $70^{\circ} \mathrm{C}$ overnight for chemical vapor deposition (CVD) of APTES. Following amine monolayer formation, the glass slides were removed from the desiccator and rinsed with toluene (HPLC grade, VWR, the Netherlands) and dichloromethane (DCM, VWR). The glass slides were then cured for at least 1 hour in the oven at $70^{\circ} \mathrm{C}$. Next, the glass slides were immersed in $0.1 \mathrm{M} \mathrm{1,4-phenylene}$ diisothiocyanate (PDITC, TCI Chemicals Belgium) in anhydrous toluene (max $0.002 \% \mathrm{H}_{2} \mathrm{O}$, VWR) for 2 hours under argon atmosphere to yield isothiocyanate bearing layers. Following the immersion, the surfaces were rinsed with toluene and DCM and incubated in $0.72 \mathrm{mM}$ heptakis amino $\beta$-cyclodextrin ( $\beta$-CD, Cyclodextrin Shop, the Netherlands) in aqueous solution at $\mathrm{pH} 8.0$ (reached by adding small amounts of $1 \mathrm{M} \mathrm{NaOH}$ ). This incubation was carried out for at least 2 hours. Surfaces were then rinsed with DI water and dried with nitrogen. 


\section{PDMS replica fabrication (PDMS microchannel features)}

A 3D positive print with channel dimensions of $20 \times 2 \times 0.5 \mathrm{~mm}$ was designed in SketchUP (Trimble) and printed with an LCD 3D printer (Photon Anycubic). For an easy peeling of the PDMS, the 3D printed molds were coated with trichloro $(1 \mathrm{H}, 1 \mathrm{H}, 2 \mathrm{H}, 2 \mathrm{H}$ perfluorooctyl)silane (PFOTS, 97\%) using chemical vapor deposition (CVD) in a vacuum desiccator; the 3D printed structure was air plasma activated for $30 \mathrm{~s}$, then it was placed in a desiccator with a vial of $100 \mu \mathrm{L}$ of PFOTS. The desiccator was then left under static vacuum for overnight CVD of PFOTS. After deposition in the desiccator, the 3D printed structure was removed from the desiccator and left in the oven at $70{ }^{\circ} \mathrm{C}$ for $1 \mathrm{~h}$, then it was washed with ethanol and isopropanol. PDMS replicas were prepared by pouring PDMS mixture (10:1 PDMS/curing agent) on the 3D printed PFOTS coated mold. After overnight curing at $70{ }^{\circ} \mathrm{C}$, the PDMS was cut out the mold and sonicated in ethanol for 4 minutes for removing small molecular weight and unreacted PDMS chains. The PDMS replica was then dried with nitrogen. An inlet and outlet were then created on the edges of the microchannel features by puncturing through the PDMS with a $1.5 \mathrm{~mm} \varnothing$ punch (KAI, Japan).

\section{Plasma microcontact patterning $(\mathrm{P} \mu \mathrm{CP})$}

PDMS stamps for $\mathrm{P} \mu \mathrm{CP}$ were fabricated using the standard technique from Whitesides. ${ }^{33}$ A silicon master was first fabricated by spin-coating at $1500 \mathrm{rpm}$ with SU-8 2025 photoresist (Microchem, USA) to yield a $50 \mu \mathrm{m}$ thick photoresist layer and then treated using UV photolithography with a photomask containing $150 \mu \mathrm{m}$ patterns and $50 \mu \mathrm{m}$ pattern spacing. The silicon master and a separate glass vial containing $100 \mu \mathrm{L}$ of trichloro $(1 \mathrm{H}, 1 \mathrm{H}, 2 \mathrm{H}, 2 \mathrm{H}-$ perfluorooctyl)silane (PFOTS, 97\%) were introduced into a desiccator under vacuum for overnight CVD. After incubation with PFOTS, the wafer was cleaned with isopropanol and dried with nitrogen. Stamps were prepared by casting a 10:1 (w/w) mixture of poly(dimethylsiloxane) (PDMS) and curing agent (Sylgard 184, Dow Corning) onto the silicon master with $150 \mu \mathrm{m}$ patterns and $50 \mu \mathrm{m}$ pattern spacing. After overnight curing at $70{ }^{\circ} \mathrm{C}$, the PDMS stamps were cut out the master to ca $0.75 \mathrm{~cm}^{2}$ and sonicated in ethanol to remove low molecular weight PDMS. The PDMS stamps were then brought into conformal contact with the freshly prepared functionalized glass surfaces. $\beta-\mathrm{CD}$ covered slides were then placed in the 
plasma cleaner (using the settings mentioned in the instrumentation section) for 4 cycles of 1 minute each.

\section{Microfluidic channel fabrication}

The PDMS replica was placed facing upwards in the plasma oven along with the $\mathrm{P} \mu \mathrm{CP}$ $\beta$-CD surface for the $4^{\text {th }}$ plasma cycle. The PDMS stamp was removed from the patterned $\beta$ CD surface and the PDMS replica was firmly placed on top of the patterned glass slide, with the microchannel facing downwards. Microfluidic device was incubated in the oven at $70{ }^{\circ} \mathrm{C}$ for at least 1 hour. Tubing was then connected to the inlet and outlet of the channel. The inlet tubing was then connected to a syringe pump and the outlet tubing to a waste vial.

\section{Bacteria binding experiments}

Before using the $\beta-C D$ functionalized microfluidic device for immobilization experiments, the microchannels and surface was thoroughly rinsed with DI water. UBI-Ad 2 was synthesized as mentioned previously. ${ }^{29}$ To functionalize $S$. aureus and E. coli with UBI-Ad 2 , $10^{8}$ CFUs were added to $1 \mathrm{~mL} 25 \mathrm{mM}$ ammonium acetate buffer $\mathrm{pH} 5$ containing $8 \mu \mathrm{M}$ UBI$\mathrm{Ad}_{2}$ and $1 \mu \mathrm{M}$ Hoechst 33342 and incubated with shaking at $37^{\circ} \mathrm{C}$. Thereafter, bacteria were centrifuged at 10,000 RCF for 5 minutes, supernatant discarded, and the pellet resuspended in phosphate-buffered saline (PBS; $10 \mathrm{mM} \mathrm{Na}_{2} \mathrm{HPO}_{4}, 1.8 \mathrm{mM} \mathrm{KH}_{2} \mathrm{PO}_{4}, 137 \mathrm{mM} \mathrm{NaCl}, 2.7 \mathrm{mM}$ $\mathrm{KCl}, \mathrm{pH}$ 7.4); such washing was repeated thrice. Bacteria were finally diluted to a concentration of $10^{8} \mathrm{CFU} / \mathrm{mL}$. Bacteria lacking the UBI-Ad $\mathrm{A}_{2}$ functionalization underwent the same procedure. For UBI-Ad 2 pre-targeting of $\beta$-CD surfaces, a $8 \mu \mathrm{M}$ solution of UBI-Ad ${ }_{2}$ in PBS was added, allowed to incubate for $1 \mathrm{~h}$ at room temperature, and then washed thrice with PBS. Binding experiments were carried out by applying flow rate of $2 \mu \mathrm{L} / \mathrm{min}$ for $1 \mathrm{hr}$ with bacteria solutions over the $\beta$-CD functionalized microfluidic device. Subsequently, microchannels were washed with PBS at a flow rate of $12 \mu \mathrm{L} / \mathrm{min}$, and images captured using confocal fluorescence microscopy. 


\subsection{Results and Discussion}

Microfluidic devices containing patterned monolayers of $\beta$-cyclodextrin $(\beta-C D)$ were fabricated as in Chapter 3. First, background interactions of bacteria with the glass surface were checked by using non-targeted $S$. aureus and E. coli (Fig. 5.1a(i)). Secondly, S. aureus and $E$. coli bacteria were pre-targeted with $\mathrm{UBI}-\mathrm{Ad}_{2}$ and then flowed separately through microchannels (Fig. 5.1a(ii)). The UBI-Ad 2 peptide interacts with the bacterial cell membrane through hydrophilic and hydrophobic interactions, therefore the peptide is sequestered within the cell membrane with Ad groups protruding from the cell surface. Moreover, we carried out pre-functionalization of the $\beta$-CD surface with UBI-Ad $\mathrm{Ad}_{2}$ and subsequently flowing a solution of unaltered bacteria over, essentially, a UBI patterned surface (Fig. 5.1a(iii)). The bacteria binding density (based on the fraction of a surface covered with bacteria) on the CD functionalized surface was also determined.In order to quantitatively analyze differences within binding experiments, the ratio of bacteria adhered on $\mathrm{CD}$ functionalized surface compared to non-CD surface was determined (also called 'binding specificity ratio'). Fig. 5.2 shows all the results and quantitative analysis of the bacteria binding experiments; we first discuss immobilization results with wild type $S$. aureus, then UBI-Ad $\mathrm{Ad}_{2}$ pre-targeted bacteria, then the effect of pre-functionalizing the glass surface with $\mathrm{UBI}-\mathrm{Ad}_{2}$ prior to bacteria immobilization. Later, we discuss the same bacterial targeting experiments for E. coli. 

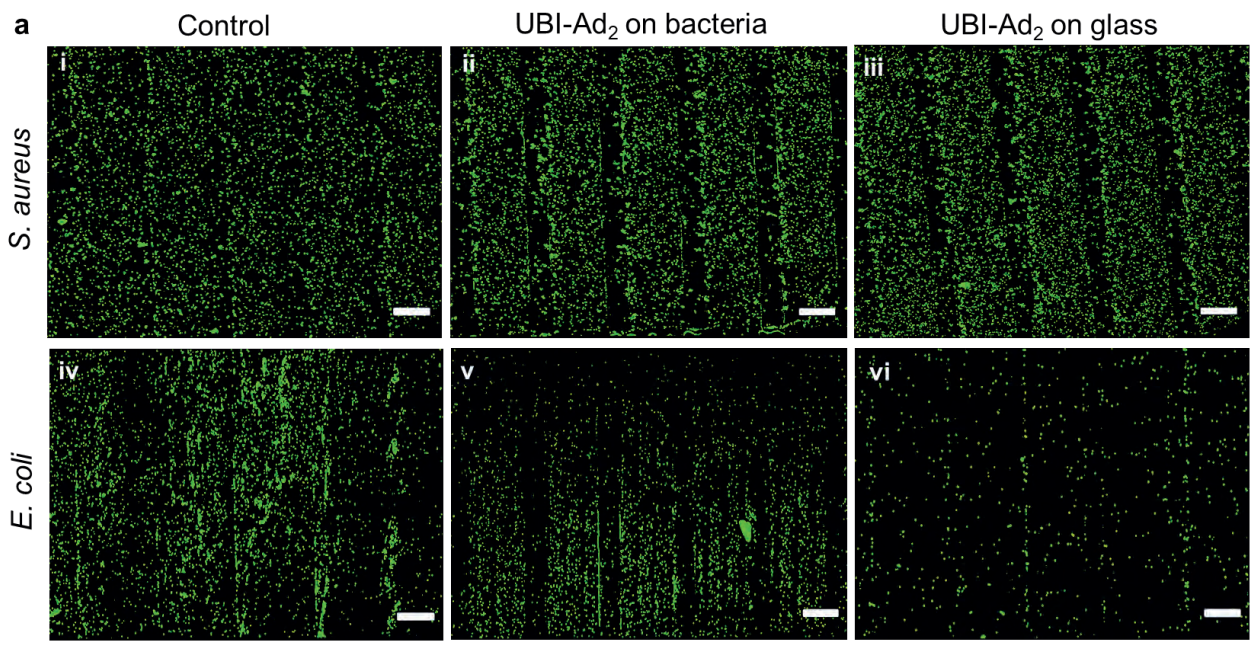

b

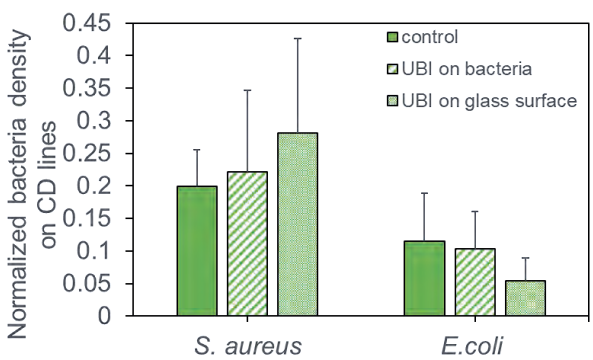

Type of bacteria
C

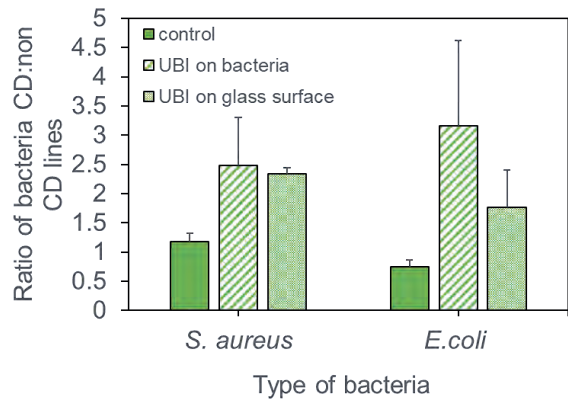

Fig. 5.2: a) Microscopy images of $\beta-C D$ patterned surfaces after $2 \mu \mathrm{L} / \mathrm{min}$ addition of $S$. aureus (i-iii) and $E$. coli (iv-vi) without UBI targeting (i,iv), UBI-Ad ${ }_{2}$ targeting on bacteria cell surface (ii,v), and on a pre-functionalized UBI surface (iii,vi). Scale bars for all images represent $100 \mu \mathrm{m}$. b. c) Graph showing the normalized density of bacteria captured on CD surface with no UBI targeting (control) samples, UBI targeting on bacteria (UBI on bacteria) or bacteria captured on pre-functionalized UBI patterned surfaces (UBI on glass surface). ) Graph showing the ratio of $S$. aureus or $E$. coli captured on CD: non-CD surface for the same samples.

\section{S. aureusbinding}

Wild type $S$. aureus bacteria were observed attaching to both $\beta$-CD functionalized and non-functionalized areas (Fig. 5.2a(i)). A bacterial binding density of ca 0.2 was calculated for CD lines, which is the fraction surface covered with bacteria (Fig. 5.2b). Wild type bacteria also attached with a more or less similar binding density to spacing between CD lines (Fig. 5.2a(i)), also portrayed by a binding ratio of c.a. 1 shown in Fig 5.2c. When $S$. aureus was pretargeted with UBI-Ad ${ }_{2}$ and subsequently flowed over the surface (Fig. 5.2a(ii)), the binding 
density of bacteria to $\beta$-CD was also slightly higher than 0.2 (Fig. 5.2b). However, this value was within the error range of the control binding density. Patterned binding of UBI- $\mathrm{Ad}_{2}$ targeted bacteria was also clearly observed in the microscopy image (Fig. 5.2a(ii), which correlates with the higher binding specificity ratio determined in Fig. 5.2c. Next, flowing of unmodified $S$. aureus over $\beta$-CD surfaces pre-functionalized with $\mathrm{UBI}-\mathrm{Ad}_{2}$ resulted in a binding density of approximately 0.3 (Fig. 5.2b). The image depicted in Fig. 5.2a(iii) also shows clear binding of the unmodified bacteria to UBI patterns with a binding specificity ratio of approximately 2 (Fig. 5.2c). Overall, an increase in $S$. aureus binding density is observed when going from wild type (control), to UBI-Ad 2 pre-targeting of bacteria and to pre-functionalization of $\mathrm{UBI}-\mathrm{Ad}_{2}$ on the $\beta-C D$ patterned surface (Fig. 5.2b). This shows that UBI-Ad $\mathrm{Ad}_{2}$ functionalized microfluidic devices are able to capture $S$. aureus bacteria most efficiently with a binding density of 0.3 compared to 0.2 of the control.

Wild type $S$. aureus most likely attaches to the surface via aspecific supramolecular interactions with $\beta-\mathrm{CD}$ patterns and charge interactions with non-functionalized spacing between patterns. Pre-targeting $S$. aureus with $\mathrm{UBI}_{-} \mathrm{Ad}_{2}$ slightly increases the binding density of the bacterium and the binding specificity ratio moderately increases from 1 to 2.5 compared to the wild type. Furthermore, the $S$. aureus binding density increases further and remains specific when switching to UBI functionalized glass surfaces. Through pre-targeting of bacteria with UBI-Ad 2 , it is expected that a high amount of Ad groups are presented on the bacterial cell surface for potential multivalent interactions with $\beta-C D$ units on the glass surface. This situation is therefore similar to the immobilization of adamantane functionalized PS particles on $\beta$-CD used in Chapter 4. We also expect that covering the surface of bacteria with UBI-Ad 2 hinders background interactions noticed with the non-functionalized spacing and compels targeted bacteria to interact with $\beta-\mathrm{CD}$ patterns via host-guest interactions. When prefunctionalizing the $\beta$-CD surface with UBI-Ad $2, S$. aureus immobilization also occurred mainly on patterned areas, which suggests that the non-functionalized spacing is blocked after incubation with UBI-Ad ${ }_{2}$. Therefore, we speculated that during the pre-functionalization step, UBI-Ad $A_{2}$ also attached to the non-functionalized spacing between $\beta$-CD patterns via aspecific interactions such as physisorption. In order to check this, we incubated a cyanine 5-stained variant of UBI-Ad 2 peptide over the $\beta$-CD patterned surface, which showed that the peptide also attached to the spacing between $\beta$-CD patterns (Fig. S1). This is possible due to the high positive charge of the UBI (ca $\mathrm{pK}_{\mathrm{a}} \sim 12$ ), which covers and neutralizes the negative silanol 
groups between CD patterns, and leads to blocking aspecific interactions of wild type bacteria with non-functionalized areas. As an additional control, the concentration of UBI-Ad $\mathrm{Ad}_{2}$ for functionalizing $\beta$-CD patterns was lowered to 2 and $4 \mu \mathrm{M}$ and tested with $S$. aureus (Fig. S2). Specific binding of $S$. aureus to UBI patterns was only possible when using a concentration of $8 \mu \mathrm{M}$ UBI-Ad $\mathrm{Ad}_{2}$ for pre-functionalizing $\beta$-CD patterns, suggesting that the negatively charged residual spacing between $\beta$-CD patterns is then properly filled to block aspecific interactions with bacteria.

\section{E. coli binding}

The same binding experiments were also carried out for $E$. coli bacteria. In first instance, flow incubation of wild type E. coli (Fig. 5.2a(iv)) resulted in a binding density of approximately 0.1 (Fig. 5.2b). There was also no preferential binding of $E$. coli to $\beta$-CD patterns, resulting in a binding specificity ratio less than 1 (Fig. 5.2c). After flow addition of UBI-Ad 2 targeted $E$. coli over $\beta$-CD patterns (Fig. 5.2a(v)), the binding density also remained at c.a. 0.1 (Fig. 5.2b) and the binding specificity ratio increased to 3. In Fig. 5.2a(iv), flowing of unmodified $E$. coli over $\beta$-CD surfaces pre-functionalized with UBI-Ad $\mathrm{d}_{2}$ resulted in a binding density of approximately 0.3 (Fig. 5.2b) and binding specificity ratio of 2 (Fig. 5.2c). From these results, a decrease in E. coli binding is observed when using UBI-Ad $\mathrm{d}_{2}$ pre-targeting compared to the wild type bacterium. The binding density decreases further when $\mathrm{UBI}-\mathrm{Ad}_{2}$ is pre-functionalized on the glass surface, showing an overall decrease in immobilization when using UBI molecular recognition targeting. The binding specificity ratio, however, increases through using UBI-Ad $\mathrm{Ad}_{2}$ pre-targeting or pre-functionalization on the $\beta$-CD patterned surface.

\section{Comparison of S. aureus and E. coli binding}

In general, it was observed that $S$. aureus was had a higher binding density than $E$. coli when flowed over functionalized surfaces. For both $S$. aureus and E. coli, the attachment of wild type bacteria to $\beta-\mathrm{CD}$ can be related to host-guest inclusion complexes being formed with the cyclodextrin cavity and receptors on the bacterial cell surface. ${ }^{34}$ An increase in binding specificity ratio was observed for both bacteria when pre-targeting the bacterial cell membrane 
with UBI-Ad 2 . We expect that covering the surface of bacteria with UBI-Ad $\mathrm{Ad}_{2}$ hinders background interactions with the non-functionalized spacing and compels targeted bacteria to interact with $\beta-C D$ patterns via host-guest interactions. Additionally, pre-functionalization of UBI-Ad $\mathrm{A}_{2}$ on $\beta-\mathrm{CD}$ patterned glass surfaces increased the specificity of both bacteria for line patterns. Since wild type bacteria also attached to non-functionalized spacing on glass (Fig. 5.2a(i+iv)), this suggests that pre-functionalizing glass surfaces with UBI-Ad $\mathrm{Ad}_{2}$ also blocks background interactions of bacteria.

It is possible that UBI has a lower binding affinity with $E$. coli or that some adamantane groups on the UBI peptide are shielded by pili and flagella on E. coli. Therefore, improved immobilization of $E$. coli could be achieved by targeting receptors on the pili. This was carried out in work from Voskuhl and Sankaran et al, ${ }^{22}$ where FimH receptors on the pili of E. coli were targeted by mannose to facilitate the immobilization of E. coli on surfaces functionalized with mannose groups. In our case, $S$. aureus bacteria preferably attaches to the surface compared to $E$. coli and binding to line patterns of $\beta-\mathrm{CD}$ becomes more specific when targeting

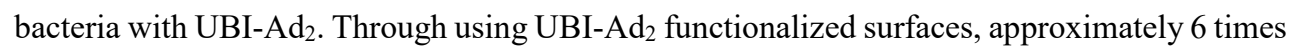
more $S$. aureus bacteria are captured compared to E. coli.

\subsection{Conclusion}

In conclusion, we used chemically modified microfluidic devices as a versatile platform for testing the on-flow immobilization of $S$. aureus and E.coli as Gram-positive and Gramnegative bacteria. We hypothesized an increase in binding of the model bacteria was achieved through formation of multivalent host-guest inclusion complexes based on adamantane and cyclodextrin. By using a diadamantane functionalized antimicrobial peptide based on a ubiquicidin segment, $\mathrm{UBI}_{29-41}\left(\mathrm{UBI}_{-} \mathrm{Ad}_{2}\right)$, adamantane guest molecules were introduced onto the bacterial cell surface prior to flow experiments over $\beta-C D$ patterned surfaces. A higher amounts of $S$. aureus bacteria were immobilized than E. coli for control and UBI-Ad 2 pretargeted bacteria samples. We also tested bacterial binding through pre-functionalizing $\beta$-CD patterns with UBI-Ad $\mathrm{Ad}_{2}$ on the glass surface. In this case, aspecific interactions of bacteria with the spacing between patterns on the glass surface were also blocked and the UBI peptide 
selectivity for $S$. aureus was further increased. In summary, surfaces with $\beta$-CD patterns created by plasma microcontact patterning $(\mathrm{P} \mu \mathrm{CP})$ allow for detailed screening revealing subtle differences in multivalent binding interactions of bacteria. Future studies will focus on more detailed quantitative analysis.

\subsection{References}

1. Rodriguez-Mozaz, S.; Chamorro, S.; Marti, E.; Huerta, B.; Gros, M.; Sànchez-Melsió, A.; Borrego, C. M.; Barceló, D.; Balcázar, J. L. Occurrence of antibiotics and antibiotic resistance genes in hospital and urban wastewaters and their impact on the receiving river. Water Research 2015, 69, 234-242.

2. Gros, M.; Marti, E.; Balcázar, J. L.; Boy-Roura, M.; Busquets, A.; Colón, J.; SànchezMelsió, A.; Lekunberri, I.; Borrego, C. M.; Ponsá, S.; Petrovic, M. Fate of pharmaceuticals and antibiotic resistance genes in a full-scale on-farm livestock waste treatment plant. Journal of Hazardous materials 2019, 378, 120716.

3. Lupo, A.; Coyne, S.; Berendonk, T. Origin and Evolution of Antibiotic Resistance: The Common Mechanisms of Emergence and Spread in Water Bodies. Frontiers in Microbiology 2012, 3 (18).

4. Bahgat, M.; Dewedar, A.; Zayed, A. Sand-filters used for wastewater treatment: buildup and distribution of microorganisms. Water Research 1999, 33 (8), 1949-1955.

5. Lüddeke, F.; Heß, S.; Gallert, C.; Winter, J.; Güde, H.; Löffler, H. Removal of total and antibiotic resistant bacteria in advanced wastewater treatment by ozonation in combination with different filtering techniques. Water Research 2015, 69, 243-251.

6. Torkelson, A. A.; da Silva, A. K.; Love, D. C.; Kim, J. Y.; Alper, J. P.; Coox, B.; Dahm, J.; Kozodoy, P.; Maboudian, R.; Nelson, K. L. Investigation of quaternary ammonium silanecoated sand filter for the removal of bacteria and viruses from drinking water. Journal of Applied Microbiology 2012, 113 (5), 1196-1207.

7. Shtarker-Sasi, A.; Castro-Sowinski, S.; Matan, O.; Kagan, T.; Nir, S.; Okon, Y.; Nasser, A. M. Removal of bacteria and Cryptosporidium from water by micelle-montmorillonite complexes. Desalination and Water Treatment 2013, 51 (40-42), 7672-7680.

8. Pinkston, K. L.; Singh, K. V.; Gao, P.; Wilganowski, N.; Robinson, H.; Ghosh, S.; Azhdarinia, A.; Sevick-Muraca, E. M.; Murray, B. E.; Harvey, B. R. Targeting Pili in Enterococcal Pathogenesis. Infection and Immunity 2014, 82 (4), 1540.

9. Romero Pastrana, F.; Thompson, J. M.; Heuker, M.; Hoekstra, H.; Dillen, C. A.; Ortines, R. V.; Ashbaugh, A. G.; Pickett, J. E.; Linssen, M. D.; Bernthal, N. M.; Francis, K. P.; Buist, G.; van Oosten, M.; van Dam, G. M.; Thorek, D. L. J.; Miller, L. S.; van Dijl, J. M. Noninvasive optical and nuclear imaging of Staphylococcus-specific infection with a human monoclonal antibody-based probe. Virulence 2018, 9 (1), 262-272.

10. Britton, K. E.; Wareham, D. W.; Das, S. S.; Solanki, K. K.; Amaral, H.; Bhatnagar, A.; Katamihardja, A. H. S.; Malamitsi, J.; Moustafa, H. M.; Soroa, V. E.; Sundram, F. X.; Padhy, A. K. Imaging bacterial infection with 99mTc-ciprofloxacin (Infecton). Journal of Clinical Pathology 2002, 55 (11), 817. 
11. Kniess, T.; Laube, M.; Wüst, F.; Pietzsch, J. Technetium-99m based small molecule radiopharmaceuticals and radiotracers targeting inflammation and infection. Dalton Transactions 2017, 46 (42), 14435-14451.

12. Summers, W. C. Bacteriophage Therapy. Annual Review of Microbiology 2001, 55 (1), 437-451.

13. Drulis-Kawa, Z.; Majkowska-Skrobek, G.; Maciejewska, B.; Delattre, A.-S.; Lavigne, R. Learning from Bacteriophages - Advantages and Limitations of Phage and Phage-Encoded Protein Applications. Current Protein and Peptide Science 2012, 13 (8), 699-722.

14. McPhee, J. B.; Hancock, R. E. W. Function and therapeutic potential of host defence peptides. Journal of Peptide Science 2005, 11 (11), 677-687.

15. Mahlapuu, M.; Håkansson, J.; Ringstad, L.; Björn, C. Antimicrobial Peptides: An Emerging Category of Therapeutic Agents. Frontiers in Cellular and Infection Microbiology 2016, 6, 194.

16. Welling, M. M.; Paulusma-Annema, A.; Balter, H. S.; Pauwels, E. K. J.; Nibbering, P. H. Technetium-99m labelled antimicrobial peptides discriminate between bacterial infections and sterile inflammations. European Journal of Nuclear Medicine 2000, 27 (3), 292-301.

17. Brouwer, C. P. J. M.; Sarda-Mantel, L.; Meulemans, A.; Guludec, D. L.; Welling, M. M. The Use of Technetium-99m Radiolabeled Human Antimicrobial Peptides for Infection Specific Imaging. Mini Reviews in Medicinal Chemistry 2008, 8 (10), 1039-1052.

18. Ferro-Flores, G.; Avila-Rodríguez, M. A.; García-Pérez, F. O. Imaging of bacteria with radiolabeled ubiquicidin by SPECT and PET techniques. Clinical and Translational Imaging 2016, 4 (3), 175-182.

19. Glukhov, E.; Stark, M.; Burrows, L. L.; Deber, C. M. Basis for Selectivity of Cationic Antimicrobial Peptides for Bacterial Versus Mammalian Membranes. Journal of Biological Chemistry 2005, 280 (40), 33960-33967.

20. Ferro-Flores, G.; Arteaga de Murphy, C.; Pedraza-López, M.; Meléndez-Alafort, L.; Zhang, Y.-M.; Rusckowski, M.; Hnatowich, D. J. In vitro and in vivo assessment of 99mTcUBI specificity for bacteria. Nuclear Medicine and Biology 2003, 30 (6), 597-603.

21. Matsuzaki, K. Control of cell selectivity of antimicrobial peptides. Biochimica et Biophysica Acta (BBA) - Biomembranes 2009, 1788 (8), 1687-1692.

22. Voskuhl, J.; Sankaran, S.; Jonkheijm, P. Optical control over bioactive ligands at supramolecular surfaces. Chemical Communications 2014, 50 (96), 15144-15147.

23. Sankaran, S.; van Weerd, J.; Voskuhl, J.; Karperien, M.; Jonkheijm, P. Photoresponsive Cucurbit[8]uril-Mediated Adhesion of Bacteria on Supported Lipid Bilayers. Small 2015, 11 (46), 6187-6196.

24. Rood, M. T. M.; Spa, S. J.; Welling, M. M.; ten Hove, J. B.; van Willigen, D. M.; Buckle, T.; Velders, A. H.; van Leeuwen, F. W. B. Obtaining control of cell surface functionalizations via Pre-targeting and Supramolecular host guest interactions. Scientific Reports 2017, 7, 39908.

25. Spa, S. J.; Welling, M. M.; van Oosterom, M. N.; Rietbergen, D. D. D.; Burgmans, M. C.; Verboom, W.; Huskens, J.; Buckle, T.; van Leeuwen, F. W. B. A Supramolecular Approach for Liver Radioembolization. Theranostics 2018, 8 (9), 2377-2386.

26. Welling, M. M.; Spa, S. J.; van Willigen, D. M.; Rietbergen, D. D. D.; Roestenberg, M.; Buckle, T.; van Leeuwen, F. W. B. In vivo stability of supramolecular host-guest complexes monitored by dual-isotope multiplexing in a pre-targeting model of experimental liver radioembolization. Journal of Controlled Release 2019, 293, 126-134.

27. Willems, S. B. J.; Bunschoten, A.; Wagterveld, R. M.; van Leeuwen, F. W. B.; Velders, A. H. On-Flow Immobilization of Polystyrene Microspheres on $\beta$-Cyclodextrin-Patterned Silica Surfaces through Supramolecular Host-Guest Interactions. ACS Applied Materials \& Interfaces 2019, 11 (39), 36221-36231. 
28. Willems, S. B. J.; Zegers, J.; Bunschoten, A.; Wagterveld, R. M.; van Leeuwen, F. W. B.; Velders, A. H.; Saggiomo, V. Plasma Etching for Fabricating Microfluidic Devices with Patterned Monolayers. ChemRxiv 2019.

29. van Leeuwen, F.W.B. and Roestenberg, M (2017). Enhancement of Pathogen Immunogenicity. Netherlands Patent No. NL2019373B1.

30. Schindelin, J.; Arganda-Carreras, I.; Frise, E.; Kaynig, V.; Longair, M.; Pietzsch, T.; Preibisch, S.; Rueden, C.; Saalfeld, S.; Schmid, B. Fiji: an open-source platform for biologicalimage analysis. Nature Methods 2012, 9 (7), 676-682.

31. Onclin, S.; Mulder, A.; Huskens, J.; Ravoo, B. J.; Reinhoudt, D. N. Molecular printboards: monolayers of beta-cyclodextrins on silicon oxide surfaces. Langmuir 2004, 20 (13), 5460-5466.

32. Willems, S. B. J.; Schijven, L. M. I.; Bunschoten, A.; van Leeuwen, F. W. B.; Velders, A. H.; Saggiomo, V. Covalently bound monolayer patterns obtained by plasma etching on glass surfaces. Chemical Communications 2019, 55 (53), 7667-7670.

33. Xia, Y.; Whitesides, G. M. Soft Lithography. Angewandte Chemie International Edition 1998, 37 (5), 550-575.

34. Bar, R.; Ulitzur, S. Bacterial toxicity of cyclodextrins: Luminuous Escherichia coli as a model. Applied Microbiology and Biotechnology 1994, 41 (5), 574-577. 


\subsection{Supplementary Figures}

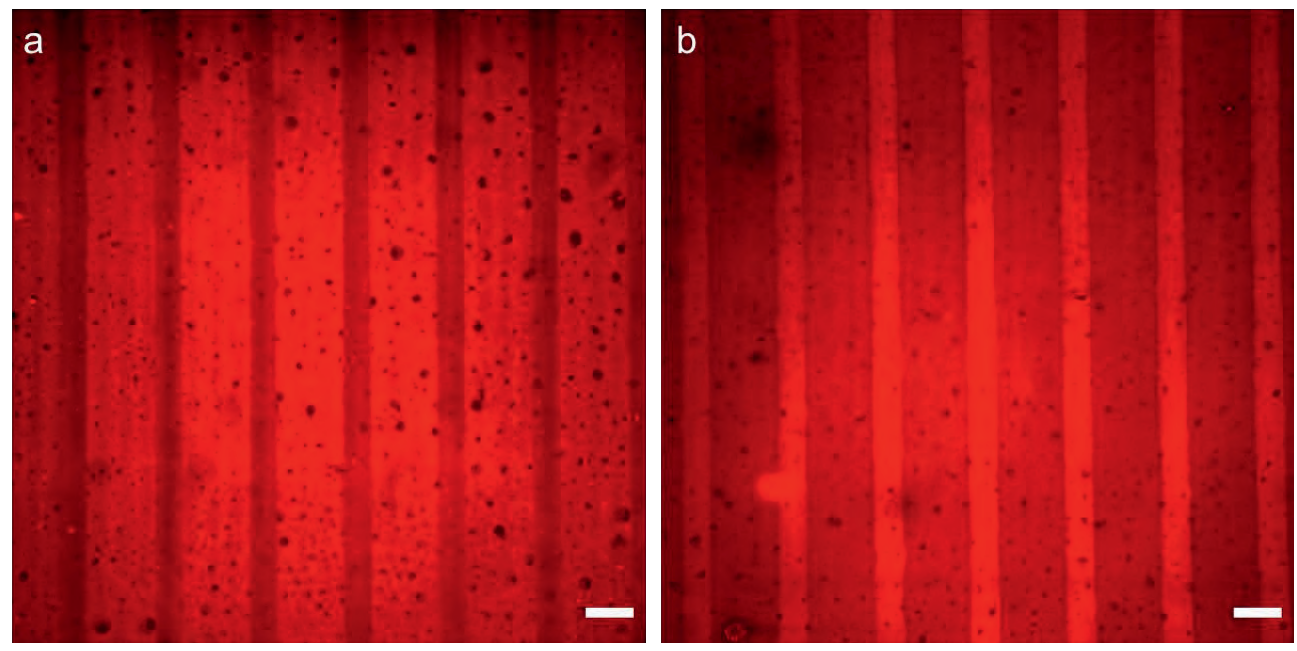

Fig. S1: Microscopy images of $\beta-C D$ patterns within microfluidic channel after addition of $1 \mu \mathrm{M}$ UBI-Cy5-Ad 2 during washing surface with PBS (a) and after running the surface dry (b). Scale bars represent $100 \mu \mathrm{m}$. 


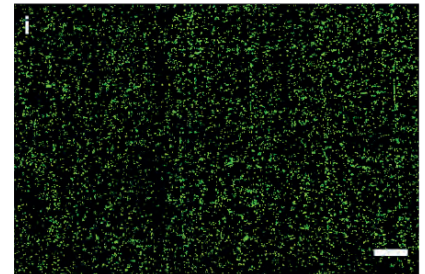

b

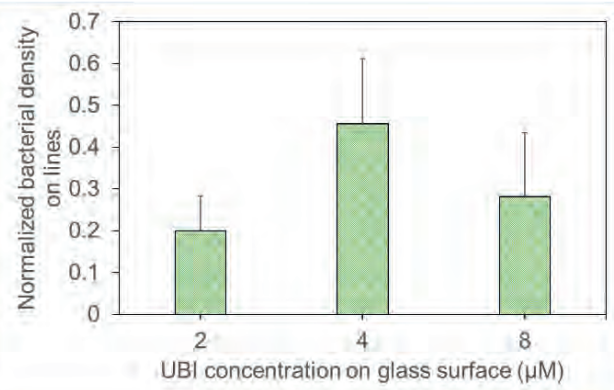

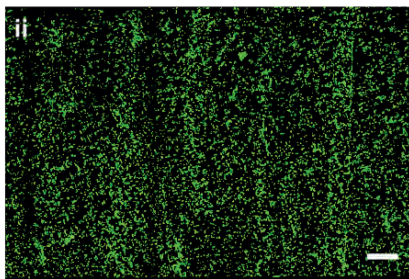

C
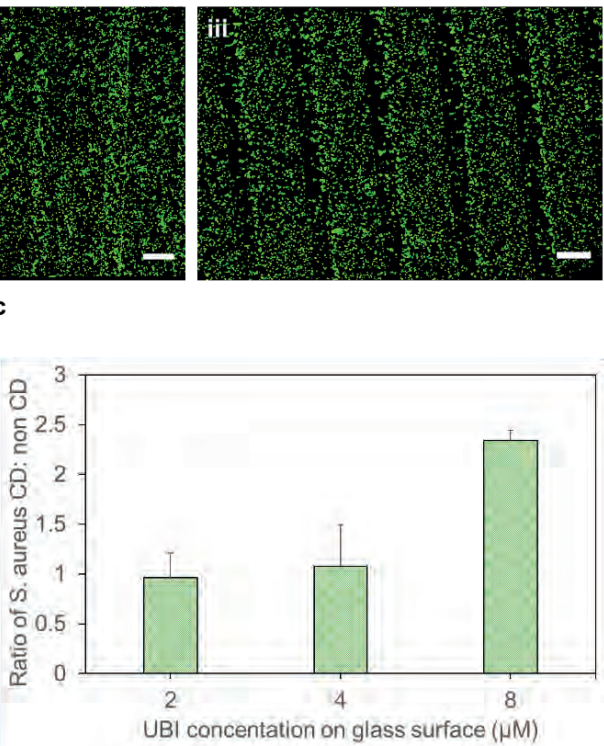

Fig. S2: a) Microscopy images after flow addition of $S$. aureus over surface pre-functionalized with a UBI-Ad 2 concentration of $2 \mu \mathrm{M}$ (i), $4 \mu \mathrm{M}$ (ii) or $8 \mu \mathrm{M}$ (iii). b) Quantitative analysis comparing the ratio of S. aureus on UBI: non UBI surface with UBI-Ad 2 concentration pre-functionalized on the glass surface. c) Graph showing the normalized bacteria density on UBI lines compared to UBI- $\mathrm{Ad}_{2}$ concentration pre-functionalized on the glass surface. 


\section{Chapter 6}

\section{General discussion}

This thesis reports on the supramolecular immobilization of (model) particles and bacteria using cyclodextrin functionalized surface incorporated within microfluidic devices. As a first step, development and characterization of functionalized surfaces in microfluidic systems was carried out, along with a focus on patterning molecules to improve analysis using microscopy. Further on in the thesis, the cyclodextrin functionalized microfluidic systems were used more practically by studying immobilization of targeted model microparticles and bacteria using host-guest chemistry. Based on these results, it can be assessed whether using host-guest chemistry is a practical tool for specific removal of bacteria within water purifications systems. In this chapter, we discuss the main findings of this thesis, other types of surface moieties which could potentially be used for cell binding applications. Importantly, the feasibility for implementation of the technology in wastewater treatment systems is discussed, with respect to improving efficiency by scaling-up, reducing fouling and antibiotic-resistant bacteria. 


\subsection{Main Findings}

Specific removal of bacteria through molecular recognition mechanisms may be considered as advanced technology for waste-water treatment. For (bio)medical and biosensing applications, labeling of receptors on surfaces through molecular recognition has been carried out for decades and has shown to be specific. It is therefore of interest to see how this concept can be translated and applied to immobilize cells such as bacteria. For this project, the goal was to prove and investigate specific targeting and capturing of bacterial cells with molecular recognition targeting, with an outlook toward scaling up the technology for use in waste-water treatment plants. A primary and important step in achieving this goal was designing a platform and setup which can be used for carrying out immobilization studies. For this, we turned to using microscopy for studying immobilization on modified glass surfaces as platforms. In the case of molecular recognition type targeting, glass surfaces were functionalized to carry out supramolecular host-guest interactions. Such interactions are known for high affinity, noncovalent and reversible binding of entities.

It can be stated that for supramolecular chemistry, host-guest inclusion complexes based on $\beta$-cyclodextrin ( $\beta$-CD, host) and adamantane (Ad, guest) are among the most popular due to high affinity binding. ${ }^{1}$ Therefore, we worked on functionalizing glass surfaces presenting $\beta-C D$ units for capturing Ad labeled constructs, which can be analyzed and confirmed using fluorescence microscopy. We argued that within microscopy using patterns of functionalized

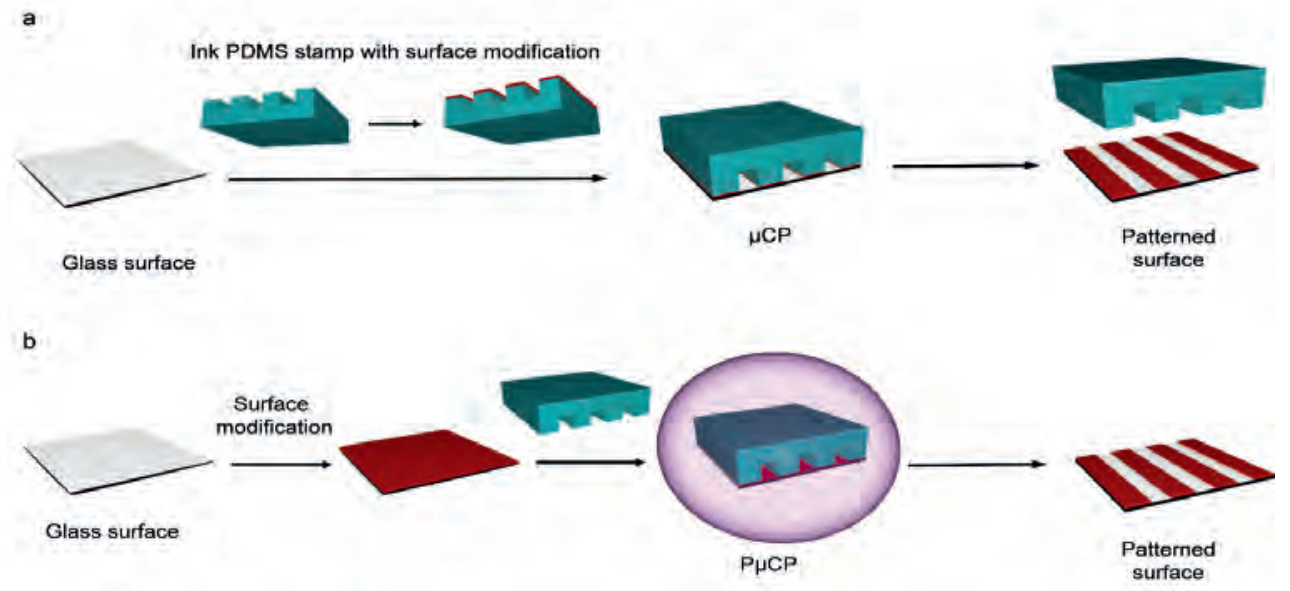

Fig. 6.1: Schematic depicting the main steps necessary for creating patterns on surface via a) microcontact printing $(\mu \mathrm{CP})$ or b) plasma microcontact patterning $(\mathrm{P} \mu \mathrm{CP})$. 
groups on the glass surface, such as $\beta$-CD units, allows for experimental control between positive, functionalized areas and negative, non-functionalized areas to improve validation with binding studies (chapter 2). Microcontact printing ( $\mu \mathrm{CP}$ ) is a cheap method for patterning surfaces and is carried out using patterned polydimethylsiloxane (PDMS) stamps for directly 'adding' molecular features to surfaces. However, it can be hampered by experimental reproducibility for first-time users and even more experienced users, ${ }^{2}$ due to its sensitivity to pressure, solvent compatibility, etc. Therefore, we explored a new methodology for pattern creation in chapter 2 known as 'plasma microcontact patterning' $(\mathrm{P} \mu \mathrm{CP})$, in which a patterned PDMS stamp partially protects a covalently functionalized surface subjected to plasma etching (see Fig. 6.1). Characteristics of the $\mathrm{P} \mu \mathrm{CP}$ technique used in this chapter include:

- Pattern creation through removing surface functionalization (in contrast to additive manufacturing)

- $\quad$ Less steps than $\mu \mathrm{CP}$

- Higher reproducibility and easier than $\mu \mathrm{CP}$ because of consistent plasma etching

- Possibility for sub-10 $\mu \mathrm{m}$ features through rotating PDMS stamp between plasma cycles

- No requirement of clean-room facilities

The patterned $\beta-C D$ glass surfaces were proven to be functional in terms of supramolecular targeting of guest molecules in a static setup. As a next step, we wanted to incorporate the modified surfaces in a microfluidic setup where a flow of liquid can be applied over the surface, which is more relatable to real-life applications such as waste-water treatment. In chapter 3 , we investigated combining $\mathrm{P} \mu \mathrm{CP}$ for obtaining $\beta-\mathrm{CD}$ patterns with fabrication of PDMS/glass microfluidic devices through replica molding since both processes require plasma etching. The part of the $\beta-C D$ functionalized glass surface which is subjected to plasma treatment during $\mathrm{P} \mu \mathrm{CP}$, within and outside the PDMS stamp area, can covalently attach to oxidized PDMS surfaces by plasma bonding. Therefore, by plasma treating both the $\beta$-CD glass surface covered with patterned PDMS stamp and a PDMS replica containing microchannel features, a microfluidic device containing $\beta-C D$ patterns was fabricated after removing the PDMS stamp and pressing glass surface and PDMS replica together (Fig. 6.2). The fabrication of microfluidic devices with covalently bound molecular patterns within microchannels as biosensors has many advantages, including: 
- measuring signal to noise ratio of analyte binding to patterns vs spacing for qualitative or quantitative purposes

- improved handling of liquids over the surface through microfluidic channels

- re-usability of devices; which was tested with Cy5-Ad2 immobilization, then removing through washing with competing agent $\beta-\mathrm{CD}$, followed by re-addition

- possibility for multiple channels over one patterned surface for micro-array based analyses, such as testing host-guest binding kinetics with different concentrations of Cy5-Ad2

\section{a}

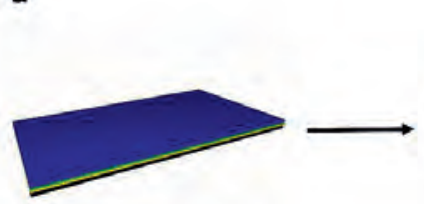

Modified surface

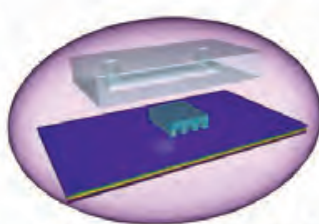

Plasma treatment

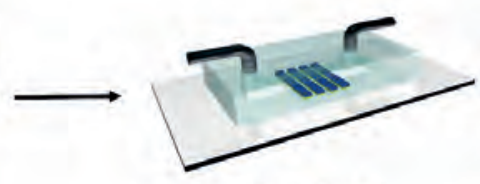

Patterned microfluidic device

b

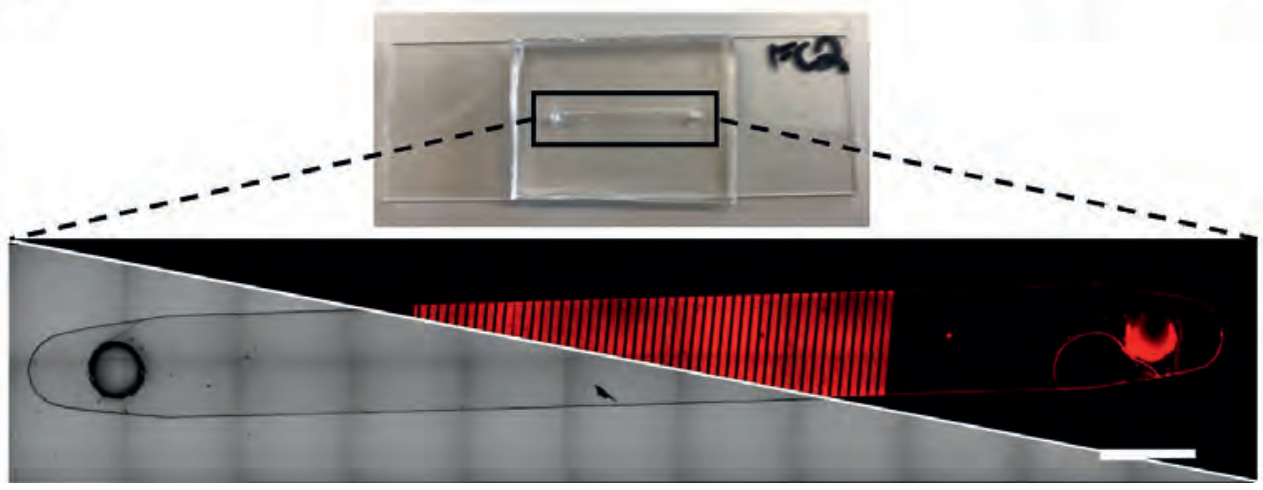

Fig. 6.2: a) Principle of combining $\mathrm{P} \mu \mathrm{CP}$ and microfluidic device fabrication to create PDMS/glass patterned microfluidic devices. b) Photograph of fabricated device and microscope images to show fluorescent patterns after addition of Cy5- $\mathrm{Ad}_{2}$. Scale bar represents $2 \mathrm{~mm}$. 
In the previous chapters, nano-sized entities such as Cy5-Ad2 were successfully immobilized through host-guest interactions on $\beta$-CD platforms using static and flow incubations. Scaling up host-guest interactions toward capturing micro-sized entities such as bacteria presents a more challenging goal for the nano-sized $\beta-\mathrm{CD}$. Therefore, we decided to test the feasibility of capturing micron-sized entities with host-guest interactions by using adamantane functionalized polystyrene microparticles as a model for bacterial cells. With an outlook toward implementing host-guest interactions for specific bacterial removal in wastewater treatment, we tested several important factors for microparticle immobilization on flow:

- variation in flow rate to determine ideal speed for achieving efficient particle capturing

- effect of adamantane loading on the polystyrene particle surface in particle capturing

- effect of settling of microparticles during flow by placing $\beta$-CD functionalized surface upside down in microfluidic device

- re-usability of $\beta$-CD patterned surface after removing PS particles

Particle immobilization on $\beta-C D$ patterned surfaces was selective for adamantane functionality, which was validated through including a control PS particle without adamantane groups (Fig. 6.3). We found that there is an optimum in particle capturing with the flow rate variation experiment: very low $(0.125 \mu \mathrm{L} / \mathrm{min})$ and very high $(200 \mu \mathrm{L} / \mathrm{min})$ flow rates result in a lower amount of captured particles. This binding profile was correlated and validated by Comsol simulations, which showed that particle settling played an important role on the particle immobilization. When the flow rate is kept the same, a higher adamantane surface coverage on PS particles increases the amount of particles captured. This proves the importance of multivalency for immobilization of micron-sized constructs using host-guest interactions. We also found that specifically bound PS particles can be removed from the functionalized glass surfaces through sonication in alcoholic solutions, and the $\beta-\mathrm{CD}$ units can consecutively be reused for particle capturing. 

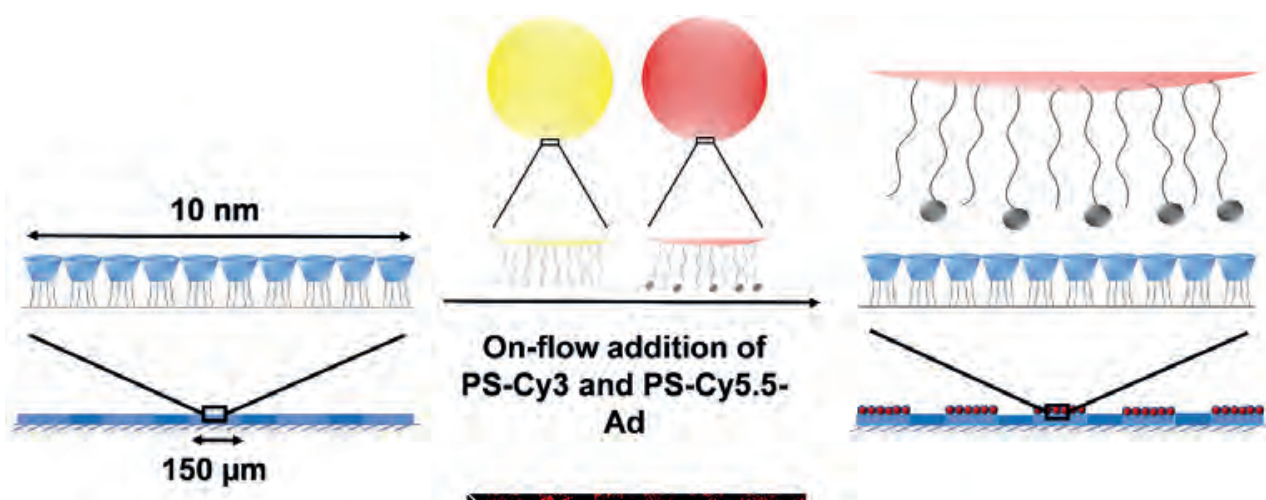

\section{On-flow addition of PS-Cy3 and PS-Cy5.5- \\ Ad}

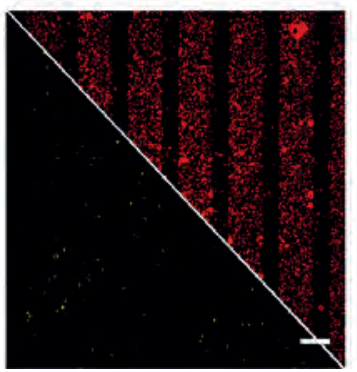

\section{Cyclodextrin specific immobilization}

Fig. 6.3: Schematic and microscopy images depicting the specific on-flow immobilization of adamantane functionalized polystyrene particles to cyclodextrin patterns on the glass surface

As immobilization of micron sized polystyrene particle was successful on the cyclodextrin functionalized surfaces, we moved to capturing $S$. aureus and E. coli bacteria. Previously, the polystyrene particle surface was chemically modified with adamantane groups. Now, we used an adamantane functionalized antimicrobial peptide, known as $\mathrm{UBI}-\mathrm{Ad}_{2}$, to target the bacterial cell surface and present it with adamantane moieties for immobilization onto $\beta-C D$ functionalized surfaces. These bacteria have roughly the same size as the polystyrene particles, and thus we hypothesized that the immobilization should be possible through hostguest interactions when assuming similar amounts of accessible adamantane units and using the same flow rates. However, the extracellular structure of bacteria, i.e. bacterial cell surface, contains many more (biological) components than polystyrene particles, such as fimbria, flagella, phospholipids, lipopolysaccharides, glycoproteins, etc. These components could either repel any kind of interaction with functionalized planar surfaces or facilitate adhesion through fouling processes. Moreover, there is also a large difference in bacterial cell envelope structure between Gram-positive and Gram-negative bacteria. Therefore, S. aureus and E. coli are 
reliable model bacteria for testing immobilization on planar surfaces via host-guest interactions. To study the immobilization of bacteria to $\beta$-CD patterned surfaces, the following experiments were carried out:

- binding of UBI-Ad 2 targeted bacteria to $\beta-C D$ patterned surfaces by applying a flow rate of either 2 or $20 \mu \mathrm{L} / \mathrm{min}$

- $\quad$ pre-functionalization of $\beta$-CD patterned surfaces with UBI-Ad2 and then flow addition of bacteria at 2 or $20 \mu \mathrm{L} / \mathrm{min}$

- varying concentration of pre-functionalized UBI-Ad $\mathrm{A}_{2}$ on surface and flow addition of $S$. aureus at $2 \mu \mathrm{L} / \mathrm{min}$

Results showed that pre-targeting of $S$. aureus with UBI-Ad 2 immobilized bacteria only onto $\beta$-CD patterns surfaces during flow incubation. Non-targeted bacteria adhered to spacing between cyclodextrin but also onto $\beta$-CD patterns which could be due the formation of inclusion

a
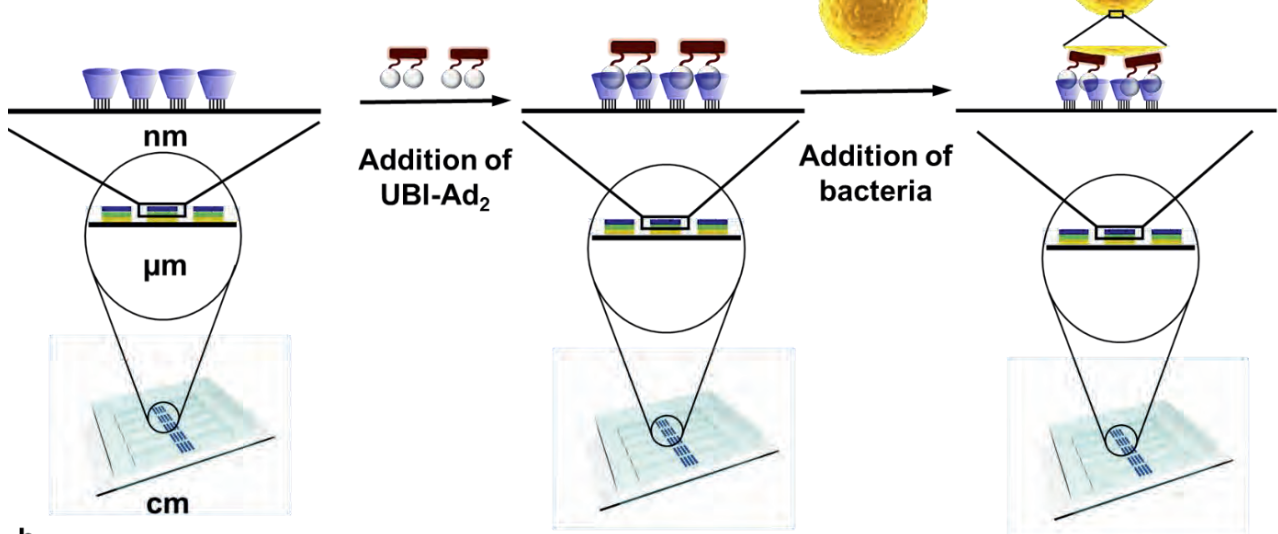

b

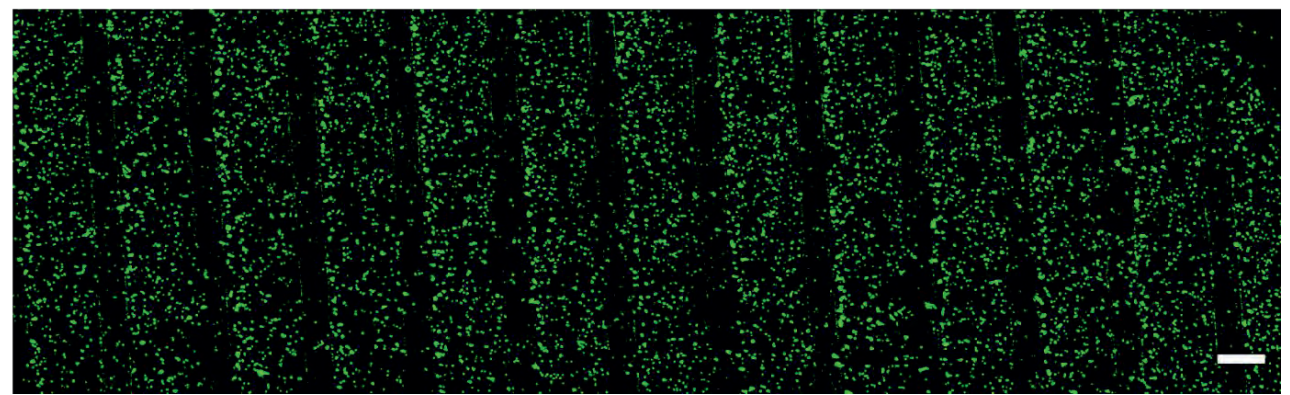

Fig. 6.4: a) Principle of immobilizing bacteria to UBI-Ad $\mathrm{Aun}_{2}$ functionalized cyclodextrin surfaces. b) Microscopy image showing the successful on-flow immobilization of $\mathrm{UBI}_{-} \mathrm{Ad}_{2}$ functionalized $\mathrm{S}$. aureus to cyclodextrin patterns. Scale bar represents $100 \mu \mathrm{m}$. 
complexes between phospholipids and cyclodextrin. ${ }^{3}$ Phospholipids constitute a large part of cellular membranes which can explain the aspecific retention of both $S$. aureus and E. coli not targeted by UBI-Ad 2 . Therefore, the results with pre-targeting bacteria with an antimicrobial peptide UBI-Ad 2 prevents the adhesion of bacteria to the spacing between $\beta-C D$ patterns. Addition of UBI-Ad 2 to $\beta$-CD functionalized surfaces prior to applying a flow of bacteria also showed a significant increase in bacteria immobilization and selectivity for S. aureus rather than E. coli (Fig. 6.4).

On the basis of the results described in this thesis, we can conclude that using supramolecular host-guest interactions based on $\beta-\mathrm{CD}$ and adamantane are promising for detection of 'large' constructs such as micrometer sized particles and bacteria. The use of microfluidics in testing immobilization of molecules, microparticles, or cell provides a versatile testing ground that is especially useful for finding the optimal retention efficiency of these entities. Furthermore, the obtained results using these model systems for capturing microsized entities from a flow of solution also determines the feasibility for scaling up toward wastewaster treatment applications. 


\subsection{Alternative Surface Functionalizations for Immobilization of Cells}

In addition to cyclodextrin functionalized surfaces, we also explored surfaces modified with lectins and heavy-metal ions for binding of micron sized cells, such as microalgae and bacteria. Such surfaces also hold promise for immobilization of cells through supramolecular binding motifs based on carbohydrate-lectin pairs or formation of metal-coordination complexes such as nickel and His6-tags.

\section{Lectin functionalized glass surface}

Lectins are carbohydrate binding proteins, each with an affinity for certain types of carbohydrates. ${ }^{4}$ The surface of cells generally contains many carbohydrates and therefore, specificity could be created in cell targeting by selecting unique lectin-carbohydrate couples, such as concanavalin A and mannose. Within our group, the aggregation of nanoparticles has been used as sensors for the sensitive detection of lectins in solution through binding of mannose functionalized quantum dots to free concanavalin A. Quantum dots were functionalized with cyclodextrin and captured adamantane modified mannose groups, which could in turn be used to capture concanavalin A and cause aggregation. ${ }^{5}$ The loss of fluorescence signal in solution due to aggregation and settling of quantum dots was measured with fluorescence spectroscopy and defined the system as a sensor for lectin detection. We therefore tested the principle of lectin binding to sugar groups on cells by using the microalgae

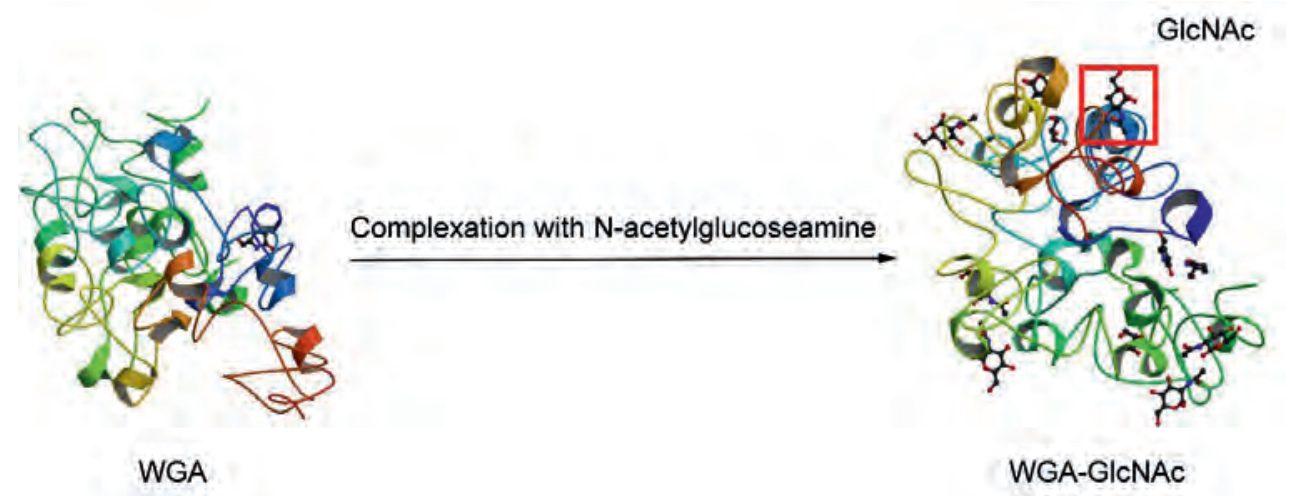

Fig. 6.5: Crystal structure of wheat germ agglutinin (WGA) lectin protein before (structure from Harata et al. ${ }^{6}$ ) and after complexation with $\mathrm{N}$-acetylglucoseamine (GlcNAc, structure from Schwefel et al. ${ }^{7}$ ). 
Nannochloropsis gaditana as another model for bacteria and functionalizing a planar silica surface with wheat germ agglutinin (WGA) lectin, which targets sialic acid or Nacetylglucosamine residues that are abundant on the algae's surface (Fig. 6.5).

We tested the functionalization of WGA on glass surfaces by using either covalent attachment of amine groups on WGA via isothiocyanate bearing layers on glass or physisorption of WGA, which is commonly carried out with proteins on glass surfaces. ${ }^{8}$ Firstly, WGA was labelled with rhodamine B isothiocyanate (RITC), and was either patterned on glass surfaces via conventional $\mu \mathrm{CP}$ or using $\mathrm{P} \mu \mathrm{CP}$. For covalent attachment of WGA on isothiocyanate functionalized glass surfaces, $\mu \mathrm{CP}$ was used similarly to printing heptakis amino $\beta-\mathrm{CD}$ and backfilling in PEG-NH 2 took place afterwards (Fig. 6.6a). Through the fluorescent labelling of WGA, the patterns could be readily analyzed by fluorescence microscopy, which validated the attachment of the lectin to the glass surface (Fig. 6.6b). After validation, flow experiments with $N$. gaditana microalgae were carried out which showed clear patterned binding of the microalgae to the lectin functionalized surface at a flow rate of $10 \mu \mathrm{L} / \mathrm{min}$.

Besides covalent attachment of WGA, physisorption of the lectin protein was also tested. Surface were fully functionalized through covalent attachment of PEG- $\mathrm{NH}_{2}$ and were

a

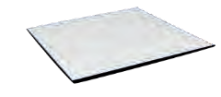

NCS functionalized surface

b

Validation of WGA patterns through fluorescence microscopy
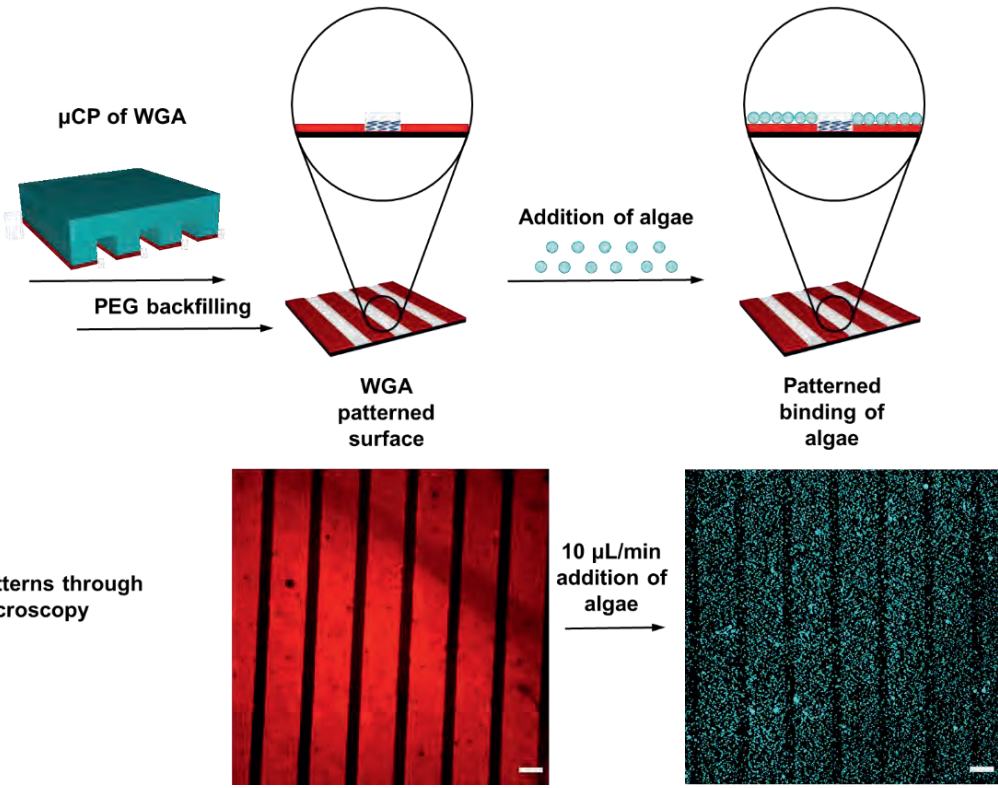

Fig. 6.6: a) Scheme depicting the procedure for $\mu \mathrm{CP}$ of rhodamine B isothiocyanate (RITC) stained wheat germ agglutinin (WGA) lectin on an isothiocyanate (NCS) functionalized glass surface and interaction of N. gaditana (algae) with the surface. b) Fluorescence microscopy images of WGA-RITC patterns using RHOD channel and WGA-RITC surface after $10 \mu \mathrm{L} / \mathrm{min}$ flow addition of $0.17 \mathrm{pM} \mathrm{N}$. gaditana algae for 30 minutes. Scale bars are $100 \mu \mathrm{m}$. 
then subjected to $\mathrm{P} \mu \mathrm{CP}$, which resulted in $150 \mu \mathrm{m}$ broad patterns of PEG on the glass surface with $50 \mu \mathrm{m}$ broad spacing of hydroxyl groups/'charged' surface. WGA was then physisorbed on the surface between PEG patterns and validated on the microscope as thinner fluorescent lines (Fig. 6.7a). For these WGA patterned surfaces, flow experiments with N. gaditana were also carried out at a flow rate of $10 \mu \mathrm{L} / \mathrm{min}$. Clear patterned binding of the algae was also visible in Fig. 6.7b.

In our experience, creating patterns of WGA through $\mathrm{P} \mu \mathrm{CP}$ of $\mathrm{PEG}$ functionalized surfaces and subsequent backfilling with WGA was more reproducible than $\mu \mathrm{CP}$ WGA on isothiocyanate bearing layers. Furthermore, control experiments in which $\mu \mathrm{CP}$ bovine serum albuminin (BSA) was carried out showed aselective adhesion of algae to printed patterns on the surface, which was not the case with $\mathrm{P} \mu \mathrm{CP}$ of BSA. Electrostatic deposition of proteins such as WGA between PEG patterns also avoids $\mu \mathrm{CP}$ of proteins with PDMS stamps, which can damage the structure of protein molecules. ${ }^{9}$ Moreover, we suspect that rigid covalent attachment of protein molecules through reacting lysine/amine groups to isothiocyanate layers on glass could also hinder optimal functioning of certain proteins. Notwithstanding, the

a

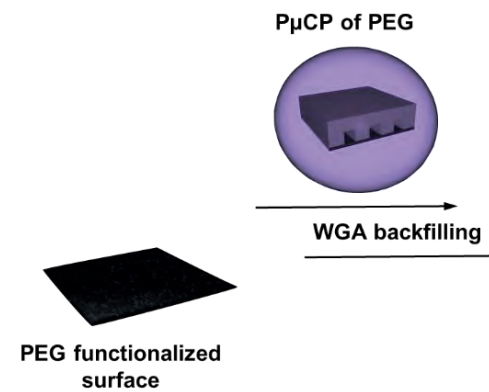

b

Validation of WGA patterns through fluorescence microscopy
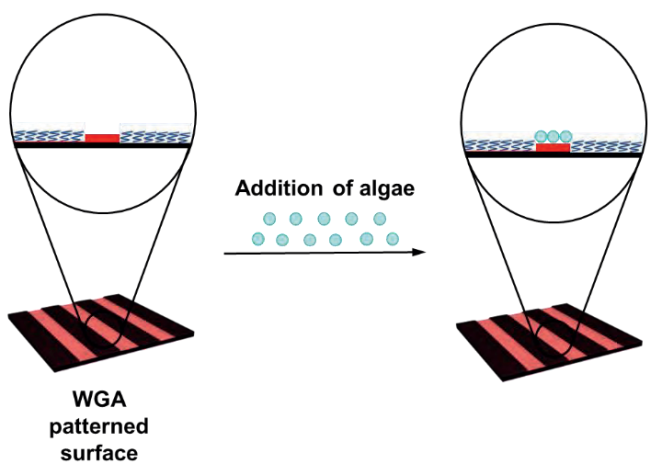

patterned
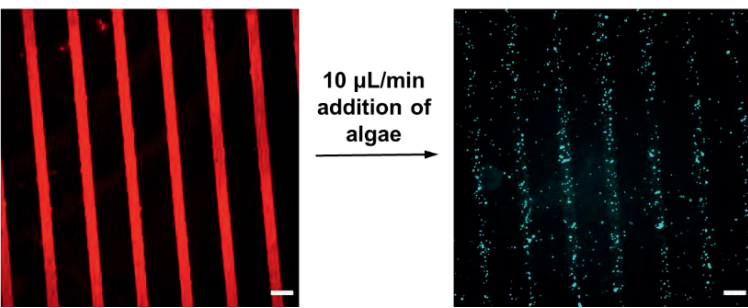

Fig. 6.7: a) Scheme depicting plasma microcontact patterning $(\mathrm{P} \mu \mathrm{CP})$ of a polyethylene glycol (PEG) functionalized surface and subsequent backfilling spacing between PEG patterns with WGA-RITC. Lastly, addition of algae over the surface for interaction with WGA. b) Fluorescence microscopy images of patterned WGA-RITC surface and patterned surface after $10 \mu \mathrm{L} / \mathrm{min}$ flow addition of $0.17 \mathrm{pM} \mathrm{N}$. gaditana algae for 30 minutes. Scale bars are $100 \mu \mathrm{m}$. 
potential of lectins for bacteria binding applications remains of interest due to the lectin's specific carbohydrate binding affinity. Lectin targeting of bacteria may be combined with $\beta$ $\mathrm{CD}$ functionalized platforms by using intermediary adamantane functionalized carbohydrates, which could improve reversibility of binding through non-covalent host-guest interactions. Considering further applications for this project, the fabrication of protein arrays can be carried out in combination with the multi-channel PDMS/glass microfluidics devices presented in chapter 3. Following $\mathrm{P} \mu \mathrm{CP}$ of a PEG functionalized surface, different proteins can be run through the different channels and 'backfill' between PEG patterns to create a (bio) sensor functionalized with multiple proteins, all in one chip.

\section{Surfaces functionalized with Nickel patterns}

Nickel functionalization and patterning of glass surfaces has previously been carried out by Wu et al, ${ }^{10}$ which starts by pre-coating the glass surface with nitrilotriacetic acid (NTA) and subsequent $\mu \mathrm{CP}$ of $\mathrm{Ni}^{2+}$ ions (Fig. 8a). Through formation of metal coordination complexes (Fig. 6.8b), the $\mathrm{Ni}^{2+}$ ions transfer from PDMS stamp to the NTA surface and remain attached to the surface. The $\mathrm{Ni}^{2+}$ ions on the surface can then form further coordination complexes with His6-tagged molecules, which allowed us to use His6-Green Fluorescent Protein (GFP) to validate patterns of Nickel through fluorescence microscopy (Fig. 6.8c(i)).

Static incubation of $S$. aureus bacteria was carried out on $\mathrm{Ni}^{2+}$ patterned glass slides which resulted in stable binding of cells over the whole surface (Fig. 6.8c(ii)). The nature of the $S$. aureus immobilization to $\mathrm{Ni}^{2+}$ ions could be caused by specific interactions with histidine rich proteins present on the bacterial cell surface, or aspecific physisorption through charge interactions. In the past, metal-coordination systems based on zinc/ $\mathrm{Zn}^{2+}$-DPA have been used successfully for targeting bacterial infections. ${ }^{11,12}$ Therefore, the use of metal-coordination complexes based on $\mathrm{Ni}^{2+}$ for immobilization of bacteria shows promise for further investigation. 


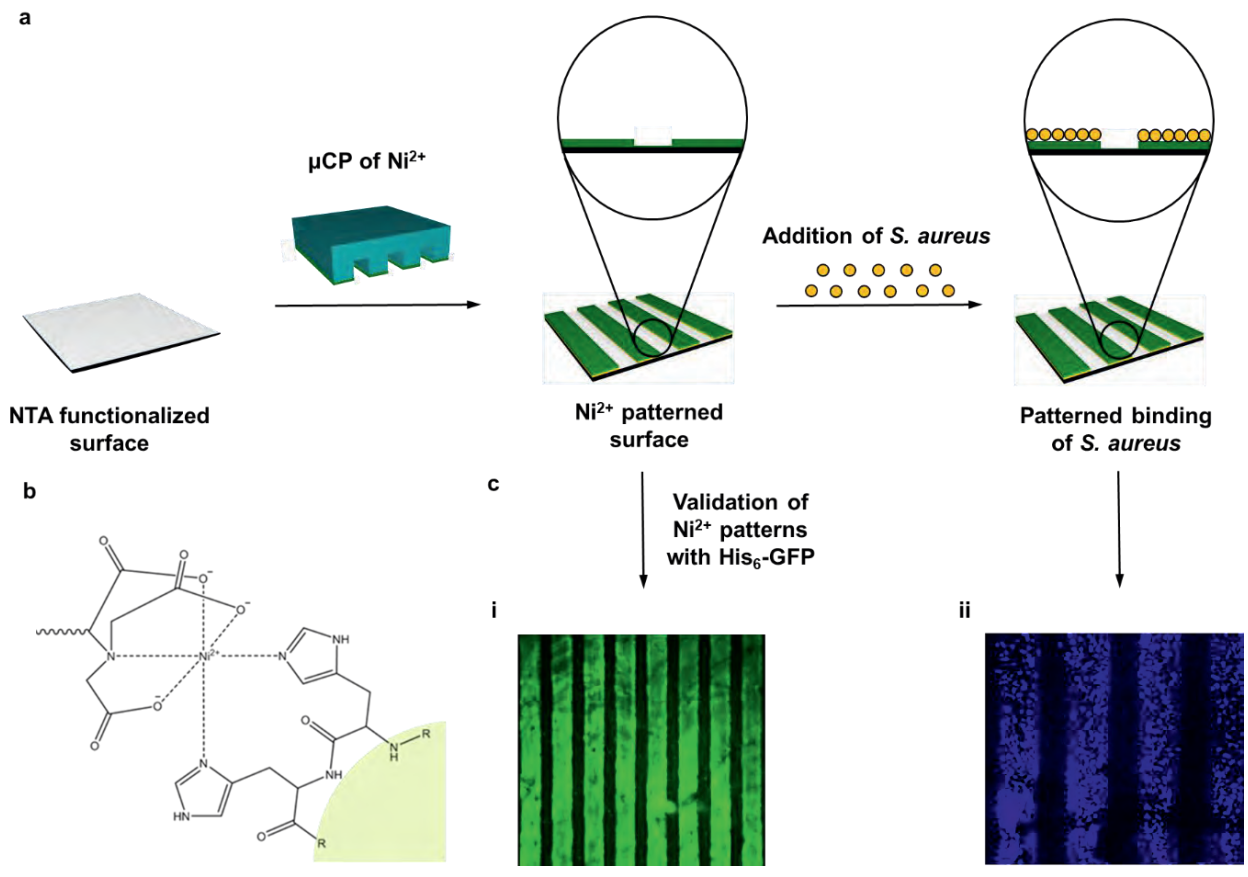

Fig. 6.8: a) Scheme depicting the procedure for $\mu \mathrm{CP}$ of $\mathrm{Ni}^{2+}$ ions on an nitrilotriacetic acid (NTA) functionalized glass surface and interaction of S. aureus bacteria with the surface. b) $\mathrm{Ni}^{2+}$-NTA interaction with $\mathrm{His}_{6}$-tagged biomolecule. c) Fluorescence microscopy images of Ni patterned surface after validation with His ${ }_{6}$-GFP (i) and after $1 \mathrm{hr}$ static incubation of $2 \times 10^{8} \mathrm{CFU} / \mathrm{mL}$ S. aureus bacteria (ii). Printed patterns in both images are $100 \mu \mathrm{m}$ broad. 


\subsection{Feasibility for Implementation in Wastewater Treatment Plants}

The microfluidic setup used for flow experiments with particles and bacteria can be considered a starting point for testing the proof-of-principle for molecular targeting and subsequent immobilization of micron sized entities to functionalized surfaces. The surface functionalization, validation and small-scale binding experiments using modified platforms are necessary preliminary steps to determine the feasibility and efficiency of specific molecular recognition in bacteria removal from wastewater. The use of cyclodextrin and diadamantane functionalized UBI peptide also provides a promising aspect for potential implementation for wastewater treatment. In addition, analysis using microscopy during binding experiments functions as a very powerful tool to determine factors such as immobilization efficiency and specificity of binding. Such experiments generate a plethora of information for future work. However, direct implementation of the described modified planar surface to act as a specific filter for bacteria removal within a wastewater treatment plant is not efficient.

Compared to large scale treatment of wastewater, relatively low flow rates $(<1 \mathrm{~mL} / \mathrm{min})$ were used during particle and bacteria immobilization experiments in order to promote settling and ensure contact of the modified particle or bacterial cell surface with the modified glass surface. The effect of particle settling was confirmed in chapter 4 by analysis of particle trajectories within Comsol simulation experiments and carrying out inverted flow incubations by turning the flow cell holder and functionalized surface upside-down. The inverted flow incubation yielded less captured particles compared to the 'regular', upright flow incubation at similar flow rates, which experimentally shows that settling of particles significantly improves binding via host-guest interactions. In contrast, spherical surfaces such as silica beads are suspended in solution and could be promising for promoting contact of bacteria to molecular recognition sites on the surface. Application of the surface functionalization steps used on glass surfaces to spherical surfaces ranging from $\mu \mathrm{m}$-mm size range, such as silica beads, can also be used to test bacteria removal efficiency from solution (Fig. 6.9). By functionalizing surfaces with $\beta$-CD groups, a versatile binding platform is created for which different types of targeting agents can be used, so long as adamantane or other groups are incorporated. The alternative surface functionalizations with lectins and heavy metals discussed in this chapter can also be applied to modifying larger spheres and scaled up to compare with cyclodextrin functionalized systems. Further steps would then include scaling up to larger experimental setups for treatment of wastewater, quantifying removal of pathogenic strains of bacteria through analysis of 

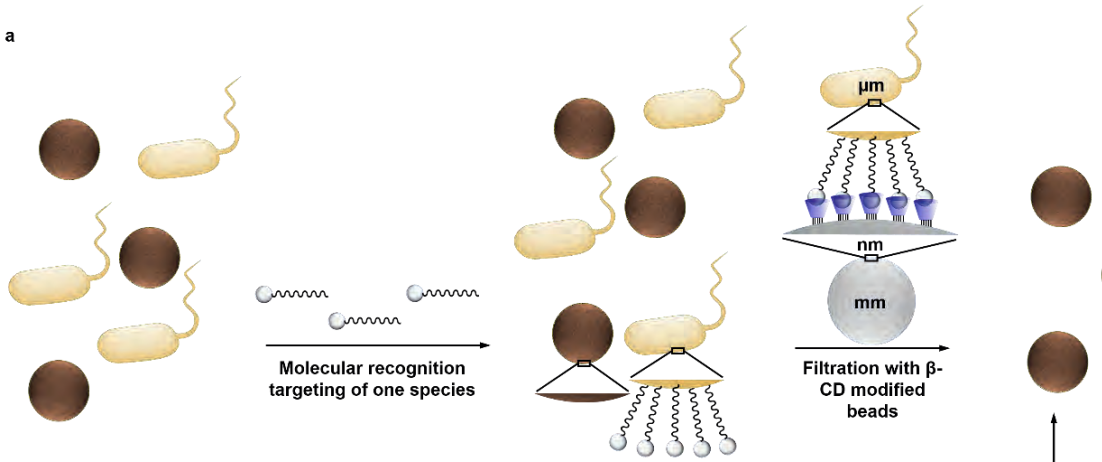

b
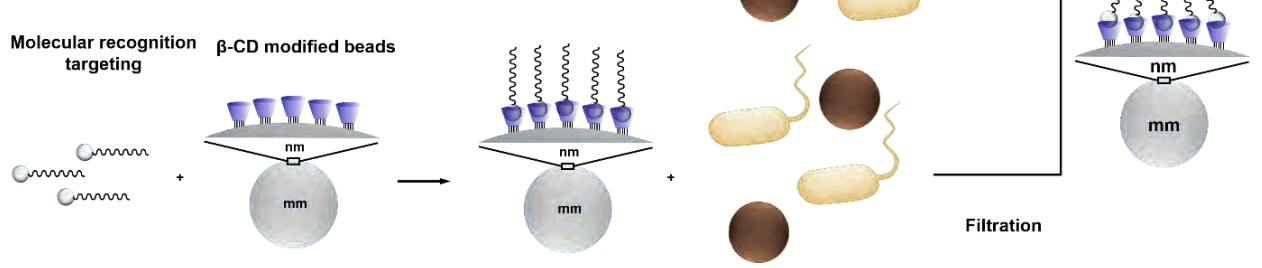

Filtration

Fig. 6.9: Scheme showing implementation of species-specific removal of micron sized entities using molecular recognition targeting techniques through a) primary pre-targeting of species or b) pre-functionalization of the mm sized beads with targeting agents As an outlook toward scaling up targeting technology, different targeting agents can be implemented depending on the type of species to be removed through using host-guest interactions with versatile $\beta-C D$ functionality on modified surfaces.

effluent, comparing cost efficiency with other water purification techniques related to bacteria removal, etc. Besides increasing the efficiency of bacteria removing filters within wastewater treatment, a problem that should not be overlooked is fouling. Cyclodextrin covered surfaces, as noticed from bacterial binding experiments carried out in chapter 5, are quite susceptible to undesired interactions with non-targeted bacteria. Functionalizing the bacteria surface with adamantane groups prevented interactions of bacteria with the spacing, improving the ratio of bacteria adhered to patterns compared to spacing. Interestingly, there was no significant improvement in the amount of bacteria attached to the cyclodextrin patterns compared to the control. It is therefore expected that fouling of other bacteria, microorganisms or (in)organic material is very likely when using cyclodextrin functionalized beads for wastewater treatment.

Problems with fouling are, of course, quite common within water purification systems. Aselective interactions of filters with organic material are the main contributing factor to fouling in wastewater treatment. Many membrane-based filters suffer from fouling, which increases operation and maintenance costs. ${ }^{13}$ Solutions to decrease the effects of fouling have 
been researched, such as applying salt based cleaning solutions to remove fouling layer from a membrane,${ }^{14}$ coating the membrane surface with chitosan solution, ${ }^{15}$ and use of electric fields. ${ }^{16}$ Rinsing of cyclodextrin surfaces after bacteria immobilization using cleaning solutions with high salt concentration, or certain buffers with more acidic or basic $\mathrm{pH}$, might remove nontargeted bacteria while leaving supramolecularly immobilized bacteria on the surface. However, the results with UBI-Ad 2 peptide pre-functionalized on a cyclodextrin patterned surface showed a high binding specificity of bacteria for patterns and high selectivity for $S$. aureus. In this case, combining the versatility of the cyclodextrin functionality and prefunctionalization of a desired specific targeting agent (Fig. 6.9b), could be promising for selective bacteria removal applications in wastewater treatment. This avoids direct exposure of cyclodextrin units to (in)organic material in wastewater, which could reduce fouling.

With the rise of antibiotic resistance within bacteria, research into the selective isolation of antibiotic resistant bacteria (ARB) is also an important topic. ${ }^{17,18}$ Selective targeting of ARB is a quite challenging, as antibiotic resistance is encoded within the bacterial DNA, which is inside the cell and therefore more difficult to target. For example, bacteriophages have been used for transfecting ARB with CRISPR-Cas delivery or suppressing the SOS DNA repair system to sensitize ARB to antibiotics. ${ }^{19,20}$ However, phage delivery generally requires long incubation times, usually overnight, in order to sensitize ARB for antibiotics, which is too lengthy for wastewater treatment. Moreover, addition of bacteriophages and antibiotics to bacteria is not preferential for water purification applications. Selective targeting of ARB via receptors on the cell surface using targeting agents is also not straightforward, as it is difficult to discriminate ARB from general bacteria based on the cell surface composition. In addition, ARB generally protect themselves through hindering attachment of molecules on binding domains. By analyzing $S$. aureus and E. coli, we studied bacteria binding of two well-known species, which are also commonly antibiotic resistant. Most $S$. aureus strains are resistant to $\beta$ lactam antibiotics, known as methicillin resistant $S$. aureus (MRSA), and E. coli bacteria are also receiving attention lately within food safety due to the emergence of extended-spectrum $\beta$ lactamase (ESBL) producing E. coli. As selective targeting of antibiotic resistant strains is difficult, an easier goal would be to design filters which selectively targets only Gram-positive or Gram-negative strains. In combination with a proper diagnosis of wastewater in a certain region, the bacteria with the highest amount of antibiotic strains can be identified and targeted. 


\subsection{Summarizing Main Concluding Remarks}

Supramolecular chemistry is a versatile tool, one which can also be applied for bridging the nano- and microscale as shown with the species-specific immobilization of microsized entities such as microplastics and bacteria on cyclodextrin functionalized surfaces. Multivalency is the key aspect to achieving immobilization over this length scale and through the reversible non-covalent character of host-guest interactions, a supramolecular catch-andrelease mechanism is presented for (model) particle immobilization in this thesis. Considering these advantages, the prospect for implementation of supramolecular chemistry in wastewater treatment for pathogens is promising, especially after carrying out future work in follow-up projects.

Within this thesis, we also show how that the fabrication of functionalized (bio) sensors within microfluidic devices can be powerful within analytical studies, for use in a plethora of applications within e.g. lab- or organ-a-chip type studies. The concept of using patterns of functionalized molecules on surfaces allows for both experimental control and proper qualification of analytes, ranging from molecules to particles to bacteria. 


\subsection{References}

1. Granadero, D.; Bordello, J.; Pérez-Alvite, M. J.; Novo, M.; Al-Soufi, W. Host-guest complexation studied by fluorescence correlation spectroscopy: adamantane-cyclodextrin inclusion. International Journal of Molecular Sciences 2010, 11 (1), 173-188.

2. Perl, A.; Reinhoudt, D. N.; Huskens, J. Microcontact Printing: Limitations and Achievements. Advanced Materials 2009, 21 (22), 2257-2268.

3. Bar, R.; Ulitzur, S. Bacterial toxicity of cyclodextrins: Luminuous Escherichia coli as a model. Applied Microbiology and Biotechnology 1994, 41 (5), 574-577.

4. Sharon, N.; Lis, H. Lectins as cell recognition molecules. Science 1989, 246 (4927), 227.

5. Oikonomou, M.; Wang, J.; Carvalho, R. R.; Velders, A. H. Ternary supramolecular quantum-dot network flocculation for selective lectin detection. Nano Research 2016, 9 (7), 1904-1912.

6. Harata, K.; Nagahora, H.; Jigami, Y. X-ray structure of wheat germ agglutinin isolectin 3. Acta Crystallographica Section D: Biological Crystallography 1995, 51 (6), 1013-1019.

7. Schwefel, D.; Maierhofer, C.; Beck, J. G.; Seeberger, S.; Diederichs, K.; M 1ler, H. M.; Welte, W.; Wittmann, V. Structural basis of multivalent binding to wheat germ agglutinin. Journal of the American Chemical Society 2010, 132 (25), 8704-8719.

8. Bernard, A.; Renault, J. P.; Michel, B.; Bosshard, H. R.; Delamarche, E. Microcontact Printing of Proteins. Advanced Materials 2000, 12 (14), 1067-1070.

9. Blinka, E.; Loeffler, K.; Hu, Y.; Gopal, A.; Hoshino, K.; Lin, K.; Liu, X.; Ferrari, M.; Zhang, J. X. J. Enhanced microcontact printing of proteins on nanoporous silica surface. Nanotechnology 2010, 21 (41), 415302.

10. Wu, C.-C.; Reinhoudt, D. N.; Otto, C.; Velders, A. H.; Subramaniam, V. Protein Immobilization on $\mathrm{Ni}$ (II) Ion Patterns Prepared by Microcontact Printing and Dip-Pen Nanolithography. ACS Nano 2010, 4 (2), 1083-1091.

11. White, A. G.; Gray, B. D.; Pak, K. Y.; Smith, B. D. Deep-red fluorescent imaging probe for bacteria. Bioorganic \& Medicinal Chemistry Letters 2012, 22 (8), 2833-2836.

12. Bunschoten, A.; Welling, M. M.; Termaat, M. F.; Sathekge, M.; van Leeuwen, F. W. B. Development and Prospects of Dedicated Tracers for the Molecular Imaging of Bacterial Infections. Bioconjugate Chemistry 2013, 24 (12), 1971-1989.

13. Le-Clech, P.; Chen, V.; Fane, T. A. G. Fouling in membrane bioreactors used in wastewater treatment. Journal of Membrane Science 2006, 284 (1), 17-53.

14. Mi, B.; Elimelech, M. Organic fouling of forward osmosis membranes: Fouling reversibility and cleaning without chemical reagents. Journal of Membrane Science 2010, 348 (1), 337-345.

15. Boributh, S.; Chanachai, A.; Jiraratananon, R. Modification of PVDF membrane by chitosan solution for reducing protein fouling. Journal of Membrane Science 2009, 342 (1), 97 104.

16. Wakeman, R. J.; Tarleton, E. S. Membrane fouling prevention in crossflow microfiltration by the use of electric fields. Chemical Engineering Science 1987, 42 (4), 829842.

17. Tahrani, L.; Soufi, L.; Mehri, I.; Najjari, A.; Hassan, A.; Van Loco, J.; Reyns, T.; Cherif, A.; Mansour, H. B. Isolation and characterization of antibiotic-resistant bacteria from pharmaceutical industrial wastewaters. Microbial Pathogenesis 2015, 89, 54-61.

18. Gupta, A.; Mumtaz, S.; Li, C.-H.; Hussain, I.; Rotello, V. M. Combatting antibioticresistant bacteria using nanomaterials. Chemical Society Reviews 2019, 48 (2), 415-427. 
19. Lu, T. K.; Collins, J. J. Engineered bacteriophage targeting gene networks as adjuvants for antibiotic therapy. Proceedings of the National Academy of Sciences 2009, 106 (12), 4629.

20. Yosef, I.; Manor, M.; Kiro, R.; Qimron, U. Temperate and lytic bacteriophages programmed to sensitize and kill antibiotic-resistant bacteria. Proceedings of the National Academy of Sciences 2015, 112 (23), 7267. 


\section{Chapter 7}

\section{Summary}

This chapter summarizes the main findings and outcomes of this thesis. 
Chapter 1 of this thesis introduces the main goals and concepts used for developing a platform for the potential selective capture of bacteria from wastewater. The increase in prevalence of pathogenic bacteria within wastewater streams coupled with antibiotic resistance development of certain bacterial species provided the motivation for developing selective targeting technology. The initial goals of the thesis project were listed as: immobilizing bacteria at high binding affinity to ensure quick capturing, reversibility of binding and subsequent reusability of developed platforms, and achieving specificity. To approach these goals, supramolecular host-guest chemistry was used for testing on-flow immobilization of model microparticles and bacteria to $\beta$-cyclodextrin $(\beta-C D)$ units functionalized on glass surfaces. Patterns of $\beta-C D$ were created on functionalized surfaces as experimental control for binding experiments with molecules, particles and bacteria, which were all studied using fluorescence microscopy.

In Chapter 2, a novel methodology for creating patterns of covalently functionalized molecules on glass surfaces based on plasma etching is presented. Within the development of molecular sensors, (fluorescence) microscopy can be used for studying monolayer assembly, for which spatial control of the surface modification improves validation within one-single field of view. Microcontact printing $(\mu \mathrm{CP})$ is a cheap, open-technology that has been used frequently for patterning surfaces by 'inking' elastomeric polydimethylsiloxane (PDMS) stamps with a solution of the desired molecular sensor and 'stamping' a glass surface to create patterns based on relief features of the PDMS stamp. However, it can require a lengthy troubleshooting period, due to issues such as PDMS solvent compatibility and unwanted lateral spreading of inks during the printing process. Therefore, we investigated an alternative methodology for pattern creation known as plasma microcontact patterning $(\mathrm{P} \mu \mathrm{CP})$, which works by placing a patterned PDMS stamp on a modified surface, and subjecting the surface to plasma etching. The PDMS stamp, instead of printing an inking solution, now acts as a mask to control the exposure of the chemically modified surface to the plasma, which removes organic molecules at non-protected areas of the surface. To showcase the flexibility of the methodology, pattern creation via $\mathrm{P} \mu \mathrm{CP}$ was studied at different steps in the $\beta$-CD glass surface functionalization protocol and visualized through addition of a diadamantane modified Cy5 (Cy5-Ad 2$)$. Cy5- $\mathrm{Ad}_{2}$ is immobilized on $\beta$ $\mathrm{CD}$ groups through divalent host-guest interactions and fluorescent patterns were then validated via fluorescence microscopy, which indicated successful pattern creation. We also managed to fabricate sub-10 $\mu \mathrm{m}$ features through using 'cross'-P $\mu \mathrm{CP}$, in which the PDMS stamp was rotated between plasma treatments to remove more of the surface functionalization. In 
conclusion, we believe that $\mathrm{P} \mu \mathrm{CP}$ can open the concept of micropatterning surfaces through its reproducibility and ease of use.

Creating patterns of $\beta-\mathrm{CD}$ on glass surfaces via $\mathrm{P} \mu \mathrm{CP}$ was combined with microfluidic channel fabrication through replica molding in Chapter 3. Replica molding, as ideated by G.M. Whitesides, is the fabrication of PDMS/glass hybrid microfluidic devices through plasma bonding of a PDMS replica containing micro-sized grooves to a glass surface. PDMS replica and glass surface are both plasma treated and brought into conformal contact with each other, forming a PDMS microchannel over the glass surface and sealing the microfluidic device through covalent bond formation. Importantly, plasma activating PDMS and glass yields silanol groups on both surfaces, which react to form silyl ethers when in conformal contact with each other. $\mathrm{P} \mu \mathrm{CP}$ treatment of chemically modified glass surfaces creates patterns of the desired functionality, with silanol groups between patterns and outside of the protected region on the glass surface. This allows for attaching a plasma activated PDMS microchannel to P $\mu \mathrm{CP}$ treated glass surfaces via covalent reactions between the silanol groups. Based on this utility, we were able to fabricate PDMS/glass hybrid microfluidic devices with patterns of $\beta$-CD on the glass surface covered with a PDMS microchannel. The microfluidic devices were validated by flowing a solution of $\mathrm{Cy} 5-\mathrm{Ad}_{2}$ through channels and analyzed through fluorescence microscopy. Host-guest interactions between $\beta-\mathrm{CD}$ units on the surface and $\mathrm{Cy} 5-\mathrm{Ad}_{2}$ were also reversible through washing with a competing agent, allowing for re-usability of the microfluidic devices. In addition, we also carried out analysis of different concentrations of $\mathrm{Cy} 5-\mathrm{Ad}_{2}$ on one microfluidic device by using a glass surface with $\beta$-CD patterns sealed with multiple microchannels. These results showed a clear increase of fluorescence intensity on the microscope with higher concentrations of $\mathrm{Cy} 5-\mathrm{Ad}_{2}$. All in all, combining $\mathrm{P} \mu \mathrm{CP}$ on glass surfaces and plasma bonding PDMS replicas with micro-sized grooves provides a universal methodology to fabricate versatile microfluidic devices for (bio)sensing applications.

In Chapter 4, $\beta$-CD patterned surfaces were used for the on-flow immobilization of adamantane-functionalized polystyrene (PS-Ad) microparticles as a model for bacterial cells. In chapters 2 and $\mathbf{3}$, the nanometer sized $\mathrm{Cy} 5-\mathrm{Ad}_{2}$ was immobilized successfully via divalent host-guest interactions with $\beta$-CD units on surfaces. Therefore, with an outlook toward immobilizing bacteria, PS-Ad microparticles were used as a model system to test the feasibility of using supramolecular host-guest chemistry for capturing micro-sized constructs. PS particles were modified with different degrees of Ad and glass surfaces were patterned with $\beta$-CD through $\mu \mathrm{CP}$ and backfilling with polyethylene glycol (PEG). The different Ad loading degrees 
on the PS particle surface was confirmed through ${ }^{1} \mathrm{H}$ NMR spectroscopy, which was used to measure the concentration of unreacted Ad-amine in the PS particle supernatant after coupling reactions. Subsequently, PS-Ad particles with the highest degree of Ad loading were flowed over $\beta$-CD along patterned glass surfaces along with non-functionalized PS particles at different speeds to study the effect of flow rate on particle capturing density. Clear binding of PS-Ad particles to patterns of $\beta-\mathrm{CD}$ was shown in contrast to the control. The results also showed an optimum capturing density at a flow rate of $2.5 \mu \mathrm{L} / \mathrm{min}$, and a decrease in particle capturing when flow rates were increased. However, lower flow rates than $2.5 \mu \mathrm{L} / \mathrm{min}$ also resulted in lower particle capturing density which was caused by settling of particles before reaching the $\beta$-CD patterned area within the microfluidic flow channel. The obtained binding profile was also confirmed through Comsol simulations, which clearly demonstrated the significant effect of particle settling in capturing microparticles. Flow experiments were then carried out with different Ad loading on PS particles which showed that higher Ad loading on PS particles also increased the particle capturing efficiency on $\beta-C D$ patterned surfaces, underpinning the effect of multivalency. Moreover, we showed that modified $\beta$-CD surfaces can be re-used for particle immobilization after removing bound particles. The recyclability provides a positive outlook for incorporation within water purification systems.

By using microfluidic devices from Chapter 3, bacterial cell immobilization on the $\beta$ CD modified surface applying an intermediary targeting agent was tested in Chapter 5. Staphylococcus (S.) aureus and Escherichia (E.) coli were used as models for Gram-positive and Gram-negative bacteria and targeting was carried out through using a bacterial membrane adhering ubiquicin segment modified with two adamantane groups (UBI-Ad $\mathrm{A}_{2}$. Therefore, successful targeting of bacteria with UBI-Ad $\mathrm{Ad}_{2}$ can be validated through supramolecular immobilization on $\beta-\mathrm{CD}$ patterns within microfluidic channels. After pre-targeting both bacteria with UBI-Ad 2 , Ad-functionalized bacteria and unaltered bacteria (control) were flowed over patterns of $\beta-C D$. Results showed that UBI-Ad 2 targeted bacteria mainly immobilized on $\beta-C D$ patterns and unaltered bacteria attached to both patterns and spacing on the glass surface. However, the bacterial capturing efficiency was not increased through using host-guest interactions, while specificity for $\beta$-CD patterns was higher for targeted bacteria. This suggests that pre-targeting bacterial cells with UBI-Ad 2 blocks potential background interactions with spacing between $\beta$-CD patterns. We also noticed from results that a higher amount of $S$. aureus bacteria adhered to functionalized glass surfaces compared to E. coli, which could be related to E. coli membrane components such as pili and flagella that generally block any type of 
interaction with surfaces. Next, $\beta$-CD surfaces were pre-functionalized with UBI-Ad 2 and subjected to a flow of unaltered bacteria. Again, an increased specificity of bacteria for patterns was also observed in this situation, which is strange considering control experiments with unaltered bacteria resulted in bacteria attaching over the whole surface. Pre-functionalizing UBI-Ad ${ }_{2}$ on $\beta$-CD glass surfaces resulted in a significantly higher amount of $S$. aureus than $E$. coli. It was also observed $S$. aureus immobilization on UBI functionalized surfaces was higher and more specific to patterns compared to $\beta$-CD functionalized surfaces (control), which can be seen as a positive outlook for further research into improving Gram-positive bacteria immobilization. Furthermore, it can be concluded from this chapter that $\beta-C D$ patterned microfluidic devices are versatile systems to use for specific sensing applications. Targeting agents can be easily interchanged on the $\beta-C D$ functionalized platforms as long as they are modified with guest molecules such as adamantane.

Chapter 6 provides a general discussion on the most important experimental outcomes of the thesis and shows results of some other experimental work that was carried out throughout the project. An outlook and suggestions are given for future research lines, especially toward the goal of implementing chemically modified platforms for large-scale, specific removal of bacteria from wastewater. 


\section{Acknowledgements}

Last, but definitely not least, we have arrived at the acknowledgements section. Carrying out a $\mathrm{PhD}$ project is not something I could have done all by myself and it is through this section that I would like to thank people specifically and reminisce on the great times we had. I hope I don't forget to mention anyone who helped me.

First of all, I would like to thank my promotor and co-promotors for their support during my $\mathrm{PhD}$. Aldrik, thanks a lot for giving me the opportunity to carry out this $\mathrm{PhD}$ project and helping me all these past years! We got to know each other during my MSc thesis in 2014 at which time I already enjoyed being part of the BNT group. After my MSc thesis you helped me find an internship at Aurion and considered me during the recruitment phase of this $\mathrm{PhD}$ project when my work at Aurion was finished. I am grateful to you for believing in me all this time and for providing feedback during the many meetings we had, especially in the final year of my $\mathrm{PhD}$. It was sometimes hard to understand why you were so meticulous over certain results, but, in hindsight, this attribute helped improve the quality of my publications and experimental chapters within my PhD thesis. Vittorio, you joined the team as co-promotor when you finally realized how beautiful the fluorescent lines on my surfaces were. I enjoyed finely tuning parameters for experiments you suggested and it yielded nice results. Thanks to you, I was able to focus more on important experiments and we were able to reach clear agreements during progress meetings with Aldrik. Fijs, I am also grateful to you for all the advice and feedback you gave in the past years! It was always exciting travelling to Leiden for carrying out experiments or having meetings, in which you gave good suggestions for experiments, congress presentations, papers etc. It was nice that you always had a clear practical view on the goals of this $\mathrm{PhD}$ project in order to keep us on track.

Besides having (co) promotors, I was lucky to have a daily supervisor at both BNT and Wetsus. Anton, thank you so much for putting up with me past four years! You provided very useful experimental feedback and helped a lot with results analysis. I'm sorry about all the desiccators I broke when trying to open them, but I hope you realize that they are not easy to work with (which can be supported by the fact that other people started breaking them as well). 
Martijn, I enjoyed having meetings with you when I was at Wetsus, which could take quite long when we were discussing how certain equations were derived or when you were giving me a crash course on water physics. Lucia, you were only involved in the beginning of my $\mathrm{PhD}$ project, but I would still like to extend my gratitude for your help directly after my recruitment, organizing my working place at Wetsus, and your support during theme meetings!

Considering BNT, I would first like to start with thanking my ex-colleagues. Jan Bart, we both started our studies in Wageningen in 2008 (but I'm actually one year older than you), although we only really got to know each other during our PhD's about 7 years later. It then became obvious that we had quite some similarities such as our sense of humor (for the sake of the reader, I will not indulge further on the subject) bowling/drinking beer, driving like madmen, making irritable sounds, etc. You provided a lot of support either by helping me with experiments in the lab, having (scientific) discussions, or just by hanging out after work, thanks for that! Maria, I hope you haven't forgotten that I always starting singing Maria by Carlos Santana when I walked into your office or met you in the hallway. You were quite busy with finishing your PhD when I started and it's a shame we weren't able to hang out that much around that period, but I enjoyed the conversations we had. Suzanne, although you aren't an ex-colleague of mine, many people at BNT see you as part of the group. It was fun talking to you when you came over to Axis Y or CHAINS!

Now for all my previous and current office mates at BNT. Banu, you may have done your best to forget it, but you actually started with me in office 1046. As soon as you could, you got your own secretary HQ and you have been a great help in organizing meetings with Aldrik for me, helping me with important $\mathrm{PhD}$ deadlines and numerous other things. Thank you so much! Pieter, thanks for being a cool office mate for a couple of years. I enjoyed hearing your view on certain topics, except I don't agree with you on the Rheinheitsgebot ;). Rosa, it was nice having you over at BNT for a couple of months. In the beginning you were quite shy, but in the last few weeks you became part of the BNT crew! Koen, apparently you were also assigned to my office but you mostly just used it as wardrobe for your coat when you had to supervise practicals. Thanks for your help with some analysis of microscopy images, in general your high level of knowledge in microscopy, and for inviting me to your wedding this year! For my current office mates, Rebecca and Yurdanur, thank you both for putting up with me in the final months of finishing my PhD thesis. Rebecca, thanks for the nice discussions/talks we had about each other's projects and for letting me use some of your fluorescent dendrimers. Yurdanur, thank you for the Turkish delights you brought when you started in September. 
Let's move on to the rest of BNT. Camilla, I enjoyed your Italian accent and crazy holiday stories of you with Mirko in some conflict region. You were also quite helpful with DLS and reminding me of important $\mathrm{PhD}$ deadlines which you went through shortly before me. Thanks! Julia, I think it's safe to say that we usually agreed to disagree in the various discussions we had and that we could always joke about this. I really appreciated your way of giving me useful or positive feedback, thanks! Lars, I think you are a cool and reliable person. I enjoyed talking to you when you were a student and temporary employee at BNT and it's cool that you also have a passion for special beers (from Belgium)! Sander, someday you will either become an amazing Nespresso barista (lekker Sanders!) or a successful scientist ;). I enjoyed your uncanny humor and run-of-the-mill presentation during a group meeting about well, a milling device. Gerben, thanks for your great input for organizing the $\mathrm{PhD}$ trip and making sure no food goes to waste by eating everything!

I would also like to thank all the (ex)students of BNT, especially the students I supervised: Laura, Linda, Tammy, Niki, Tomas, and Thomas Jan. You all contributed to this thesis by improving the surface functionalization toolbox within the BNT lab. Martijn (aka mijnheer van Galen), I appreciated how you treated your superiors (me) with respect by shaking hands every time we ran into each other during your BSc thesis ;). It was fun hanging out with you and Laura at that time and also afterwards! As for all the other students, it was fun having you around in either the lab, coffee room, group meeting and Friday afternoon drinks!

I would like to acknowledge the Physical Chemistry and Soft Matter (PCC) group for their support throughout my whole $\mathrm{PhD}$. Ties and Sven, you guys allowed me to use the UV lithography setup countless of times (which also means a lot of SU-8), even in between your own experiments, and helped me when things did not work out. Thanks for that! Renko, thank you for helping me with zeta potential and conductivity measurements. Diana, I appreciated your kindness and helpfulness for finding the UV lamp when it was being used by others ;). Jeroen, thanks to your publication on polystyrene particles, we were able to make our own batches of particles and carry out some very nice work in chapter 4 . I also enjoyed our conversations while eating cake or appelflappen at the Dreijen on random mornings.

Now to start with acknowledging colleagues/friends of my second home, Wetsus, located to the far north in Leeuwarden. Ricardo, band-mate, office-mate, theme-mate, allround-mate, professionally I know you as a very motivated hard-worker which inspired me. I also had fun rocking with you in the atrium of Wetsus. Although our favorite music genres and guitar playing styles were different, we did share one attribute while rehearsing with the band: the ability to completely ignore the rest of the band and just play what we want at max volume. 
Fabian, I appreciated our talks and your calm way of approaching problems with solutions. Thank you for the good times we had at cafes in Leeuwarden and for helping me keep rhythm during our band sessions! Jorrit, thanks for being a great guitarist and band leader. Without you (and with people like Ricardo and me), we wouldn't have gotten anything done ;). Goncalo, also a theme-mate and band-mate of mine. Thanks for your support during theme meetings and your funky base riffs during rehearsals. Carlo, I liked your sense of humor and (loud) drumming skills. Sofia, Brahzil and Mariagiorgia, I was wondering, did it hurt when you fell from heaven and started singing with the band? Steffen, I enjoyed our conversations but less your vegan meals containing a high amount of garlic to compensate for the lack of meat. Your singing, energy and dedication to the band were also nice to experience! Maarten, I enjoyed your impeccable timing with making certain jokes. I hope you enjoyed playing the piano in the band and that you sometimes could hear yourself play through all the guitar noise ;).

I would also like to thank my other office mates from both of the offices I stayed in at Wetsus: Jaap, Paulina, Rik, Karine, Nimmy, Marianne, Chris, Erik, Louis, Hector, Nigel, Ragne and Ilse. Jaap, it was inspiring to see how calm you were throughout most of your $\mathrm{PhD}$, using frequent 'micro' breaks to take a step back and put things in perspective. I'm also grateful to you for showing me how Comsol works. Hector, cabron, Mariachi of Wetsus, I enjoyed the various references we made to 'Mexican' movies or series, insulting each other in the process, but constantly laughing about it.

Jan-Jurjen, you were assigned to help me with technical stuff at Wetsus, although I didn't get the chance to really make use of your expertise (except when you were modifying/destroying a microscope stage insert for me but then advised me after a couple of hours to just order it ;)). Nevertheless, I appreciated our conversations and intensive ping-pong matches during breaks. Gerben and Riet, thanks for preparing nice meals for me during lunch breaks, I think you were happy that I mostly ate the same things every day I was at Wetsus, namely Wetsus burgers, meatballs and schnitzels (aka 'deurmat').

As mentioned previously I also travelled to Leiden for work at the LUMC and got to work with some nice people from the IMI group. Sylvia, thanks for helping me out, supervising me in the lab....basically babysitting me, especially at the start of my $\mathrm{PhD}$ whenever I came over to Leiden. I hope I didn't get in your way too often while you were finishing work for your $\mathrm{PhD}$. Mick, you were also a great help, grabbing new batches of bacteria for my experiments, providing useful feedback during meetings and making inappropriate, but hilarious jokes. Danny, thanks for synthesis of new peptides to use for my experiments and for fun talks in the IMI lab! Clarize and Nik, you both worked tremendously hard to help me in the last months of 
my $\mathrm{PhD}$, thank you so much for carrying out all the bacteria binding experiments in the last experimental chapter of this thesis. I wish you the best of luck for finishing your PhD's! Matthias, thanks for organizing the weekly group meeting at IMI and for finding me some free spots to occasionally present my work to the IMI group. Mark, I would like to acknowledge and thank you again for synthesis of the Cy5- $\mathrm{Ad}_{2}$ that I used very often during my PhD work. I would also like to thank Fabian and Tessa for their support either for labwork or during group meetings!

I would also like to acknowledge my friends from my 'dispuut' who I spent time with during various extracurricular activities in Wageningen. These are, by last name, of course: Brinker, Brinke, Vos, Koorn, Gebbink, Ruigrok, Maessen, Tuytel, Hermeling, Sloot, Hopman, Grave, Slob, Bouwhuijzen, de Rijke, Langemeijer, de Boer, Fonk, Laan, Bousnina, Versluis, and Soet. Thanks for all the good times during my PhD and before (except for the PHD drink)! Slamat!

Now for acknowledging my family. I would like to express a high amount of gratitude to my father (Guido) and mother (Lucie) who have supported me in almost everything in life, and encouraged me to do a $\mathrm{PhD}$. Me and my father's passion for (bio)chemistry/technology also lead us to brew our own beers at home, some successful and some horrible, but after many tests we may conclude that the alcohol fermentation step was never a problem ;). Nathalie, my sister, you also motivated me to do a $\mathrm{PhD}$ by doing one yourself, quite successfully at Oxford if I may add. Daresay, you were so pre-occupied at the time that you could not even partake in activities from Oxford's prestigious rowing club. Katrien, my other sister, the way you worked so hard and long also inspired me to take more time on my own writing.

Last but not least, I would like to finish with thanking Laura. You have also been a great supporter of me during my $\mathrm{PhD}$, helping me with experiments and writing, encouraging me to eat home cooked meals (or just by cooking for me) on late evenings, or basically any evening, so I don't always order food. You are a fantastic partner and I look forward to you finishing your $\mathrm{PhD}$ as well! 


\section{About the Author}

Stan Bert Jan Willems was born on November $28^{\text {th }} 1989$ in Leuven, Belgium. He obtained his bachelor's degree in Molecular Life Sciences from Wageningen University in 2012, for which he completed a thesis in the Biological Chemistry group under the supervision of Prof. Dr. W.J.H. van Berkel, working on the

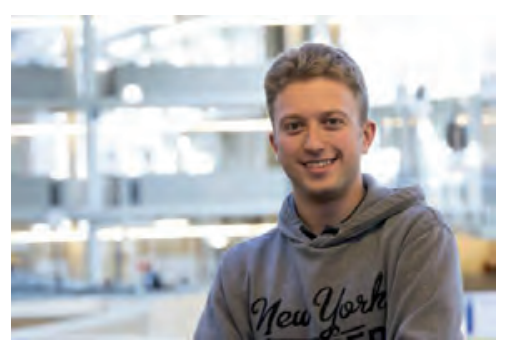
immobilization of enzymes on beads. He then obtained his master's degree in Molecular Life Sciences from Wageningen University in 2015, with a specialization in Biological Chemistry. He completed his master's thesis in the BioNanoTechnology group under the supervision of Prof. Dr. A.H. Velders, where he worked with ultrasensitive endopeptidase sensors based on multivalent gold nanoparticle networks. For his master's internship, he worked at AurionImmuno Gold Reagents \& Accessoires in Wageningen on covalent coupling of proteins to carboxylated gold nanoparticles. After obtaining his MSc degree, he continued working at Aurion as a researcher for a short time to finish work on his internship project. In 2015, he joined the Laboratory of BioNanoTechnology, Wageningen University to work on his $\mathrm{PhD}$ project, also collaborating with the Interventional Molecular Imaging laboratory, department of Radiology, Leiden University Medical Centre, and Wetsus, European Centre of Excellence for Sustainable Water Technology, in Leeuwarden. His PhD work was carried out under the supervision of Prof. Dr. A.H. Velders, Dr. V. Saggiomo and Dr. F.W.B van Leeuwen. The result of his $\mathrm{PhD}$ work is presented in this thesis. 


\section{List of Publications}

"Covalently Bound Monolayers Obtained by Plasma Etching on Glass Surfaces" Stan B.J. Willems, Laura M.I. Schijven, Anton Bunschoten, Fijs. W.B. van Leeuwen, Aldrik H. Velders, and Vittorio Saggiomo, Chemical Communications, 2019, 55, 7767-7670

"Plasma Etching for Fabricating Microfluidic Devices with Patterned Monolayers" Stan B.J. Willems, Jaccoline Zegers, Anton Bunschoten, Martijn Wagterveld, Fijs. W.B. van Leeuwen, Aldrik H. Velders and Vittorio Saggiomo. ChemRxiv, 2019. Manuscript in preparation

"On-Flow Immobilization of Polystyrene Microspheres on $\beta$-Cyclodextrin Patterned Silica Surfaces through Supramolecular Host-Guest Interactions" Stan B.J. Willems, Anton Bunschoten, Martijn Wagterveld, Fijs. W.B. van Leeuwen, and Aldrik H. Velders, ACS Applied Materials and Interfaces, 2019, 11 (39), 36221-36231

"Supramolecular Immobilization of Bacteria in Microfluidic Systems In Presence or Absence of an Adamantane Functionalized Peptide" Stan B.J. Willems, Nikolas Duszenko, Clarize M. de Korne, Anton Bunschoten, R. Martijn Wagterveld, Vittorio Saggiomo, Fijs W.B. van Leeuwen, and Aldrik H. Velders. Manuscript in preparation 


\section{SENSE}

Netherlands Research School for the

Socio-Economic and Natural Sciences of the Environment

\section{I P L O M A}

for specialised PhD training

The Netherlands research school for the Socio-Economic and Natural Sciences of the Environment

(SENSE) declares that

\section{Stan Bert Jan Willems}

born on 28 November 1989 in Leuven, Belgium

has successfully fulfilled all requirements of the educational PhD programme of SENSE.

Wageningen, 7 February 2020

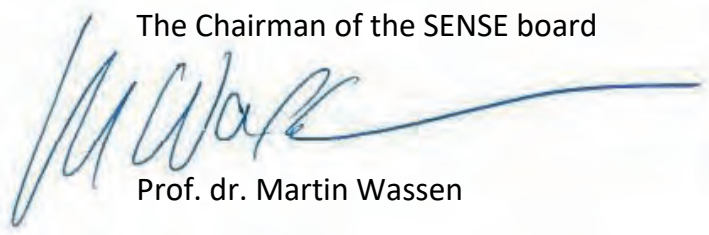

the SENSE Director of Education

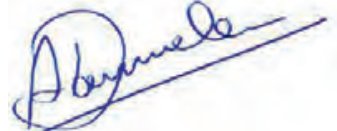

Dr. Ad van Dommelen

The SENSE Research School has been accredited by the Royal Netherlands Academy of Arts and Sciences (KNAW) 
The SENSE Research School declares that Stan Bert Jan Willems has successfully fulfilled all requirements of the educational PhD programme of SENSE with a work load of 31.7 EC, including the following activities:

\section{$\underline{\text { SENSE PhD Courses }}$}

- Environmental research in context (2016)

- Research in context activity: 'Co-organiser of PhD trip to Denmark and Sweden' (2018)

\section{Other PhD and Advanced MSc Courses}

- Laser scanning microscopy, Leiden University Medical Center (2016)

- Communication with the media and general public, Wageningen Graduate Schools (2016)

- Scientific publishing, Wageningen Graduate Schools (2017)

- Brain training, Wageningen Graduate Schools (2017)

- Competence Assessment, Wageningen Graduate Schools (2019)

o Searching and organising literature Wageningen Graduate Schools (2019)

\section{Management and Didactic Skills Training}

- Supervising two MSc students with thesis entitled 'Multi-active surface to entrap methicillin-resistant Staphylococcus aureus' (2016) and 'Metal-ion affinity system to entrap resistant bacteria' (2017)

- Supervising two BSc student with thesis entitled 'Capturing microparticles and molecules on Cyclodextrin patterned surfaces' (2017) and 'Immobilization of S. aureus on metal ions and aptamer functionalized surfaces' (2019)

0

\section{Oral Presentations}

- Immobilization of polystyrene microparticles on cyclodextrin patterned surfaces. CHAINS, 3-5 December 2018, Veldhoven, The Netherlands

- Capturing microparticles with nanogrippers: a tool against antibiotic resistance, Wetsus internal congress, 30 November 2017, Leeuwarden, The Netherlands

- 4 presentations at companies in Denmark and Sweden on 'Immobilization of model microparticles and algae on patterned surfaces"(2018)

\section{Selection of Poster Presentations}

- Immobilization of microspheres with Nanogrippers, CHAINS , 4-6 December 2017, Veldhoven, The Netherlands and ISMSC, 2-6 June 2019, Lecce, Italy

- Direct universal table-top and cheap surface treatment allowing microcontact patterning open technology, ISMSC, 2-6 June 2019, Lecce, Italy

SENSE coordinator PhD education

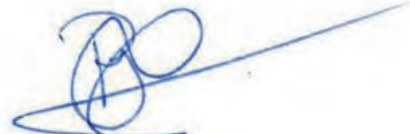

Dr. ir. Peter Vermeulen 
This work was performed in the cooperation framework of Wetsus, European Centre of Excellence for Sustainable Water Technology (www.wetsus.nl). Wetsus is also co-funded by the Dutch Ministry of Economic Affairs and Ministry of Infrastructure and Environment, the European Union Regional Development Fund, the Province of Fryslân, and the Northern Netherlands Provinces. The author would like to thank the participants of the research theme Source Separated Sanitation for the fruitful discussions and their financial support.

Cover design by Laura Schijven and the author

Printed and layout by Proefschriftmaken.nl 


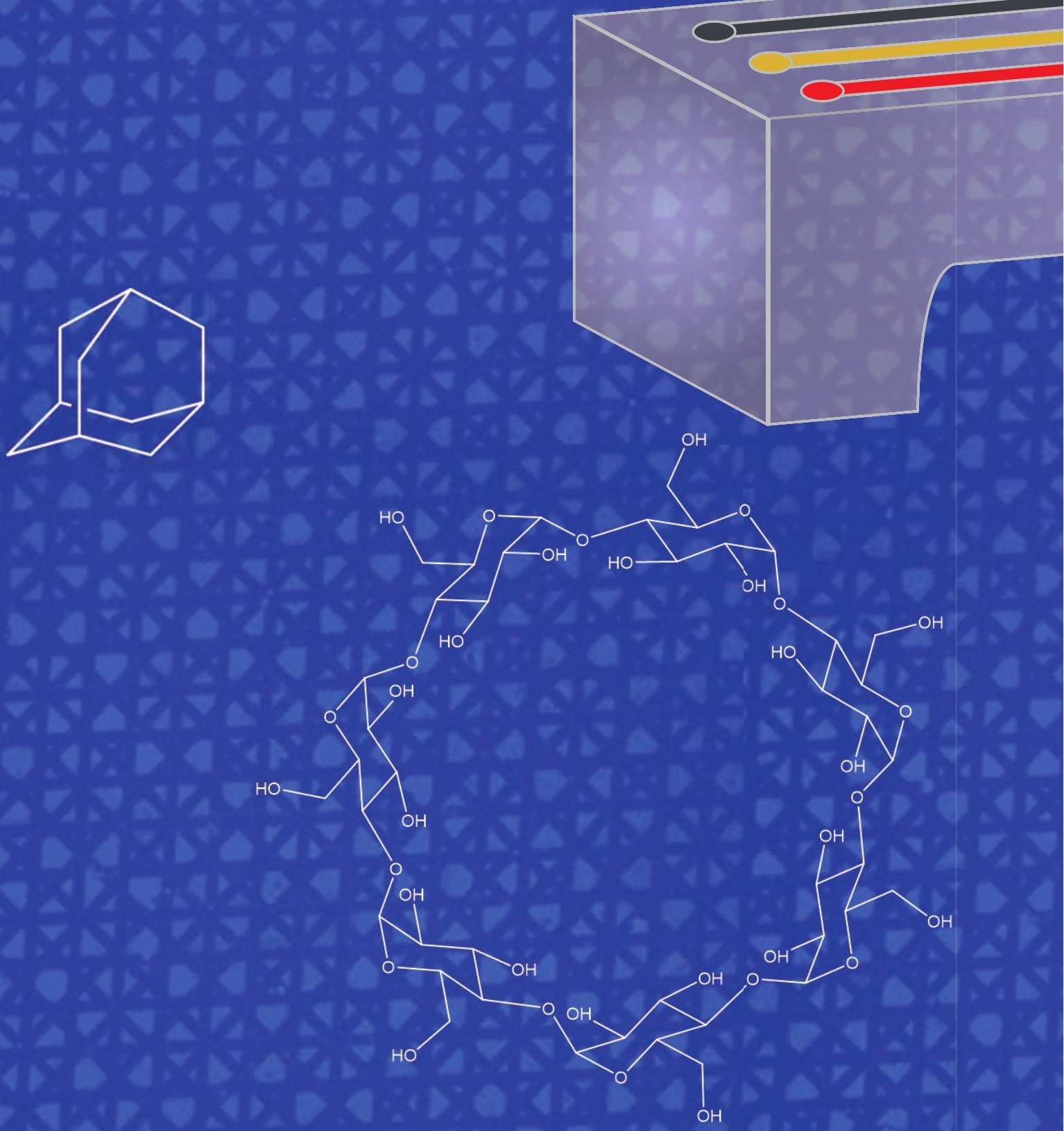
